\title{
DISTAL RADIUS FRACTURES: \\ WHAT DETERMINES THE \\ OUTCOME AFTER SURGERY?
}

\section{DISTALE RADIUS FRACTUREN:}

WAT BEPAALT DE UITKOMST

NA EEN OPERATIE?

(met een samenvatting in het Nederlands)

\section{PROEFSCHRIFT}

ter verkrijging van de graad van doctor aan de Universiteit Utrecht op gezag van de rector magnificus, prof.dr. G.J. van der Zwaan, ingevolge het besluit van het college voor promoties in het openbaar te verdedigen op donderdag 25 augustus 2016 des middags te 4.15 uur

door

Teun Teunis

geboren op 20 juli 1989 te Veghel

Promotoren: $\quad$ Prof. dr. M. Kon

Prof. dr. D. Ring

Copromotor: Dr. A.H. Schuurman 
Work performed at University Medical Center Utrecht, Utrecht University and Massachusetts General Hospital, Harvard Medical School.

The research fellowship was supported by stipends from the Massachusetts General Hospital Hand \& Upper Extremity Service, Foundation de Fundatie van de Vrijvrouwe van Renswoude te 's-Gravenhage and the Prince Bernhard Culture Fund \& Kuitse Fund.

The publication of this work was financially supported by the Nederlandse Vereniging voor Plastische Chirurgie, Junior Vereniging Plastische Chirurgie, Department of Plastic, Reconstructive and Hand Surgery of the University Medical Center Utrecht, Nederlandse Vereniging voor Traumachirurgie, Traumaplatform Foundation, Teaching Hospital of the OLVG, Van Campen Consulting and the PATIENT+ foundation. 


\section{TABLE OF CONTENTS}

Introduction $\quad 6$

$\begin{array}{lc}\text { Abbreviations } & 16\end{array}$

PART I UNDERSTANDING THE INJURY 18

CHAPTER MELONE'S CONCEPT REVISITED: 20

3D QUANTIFICATION OF FRAGMENT DISPLACEMENT

Journal of Hand and Microsurgery

CHAPTER AO DISTAL RADIUS FRACTURE CLASSIFICATION:

GLOBAL PERSPECTIVE ON OBSERVER AGREEMENT

Journal of Wrist Surgery

PART II TREAMENT

CHAPTER NO DIFFERENCE IN ADVERSE EVENTS BETWEEN SURGICALLY TREATED REDUCED AND UNREDUCED DISTAL RADIUS FRACTURES J Orthop Trauma. 2015 Nov;29(11):521-5.

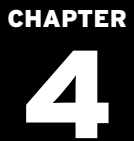

EVALUATION OF RADIOGRAPHIC FRACTURE POSITION ONE YEAR AFTER VARIABLE ANGLE LOCKING VOLAR DISTAL RADIUS PLATING: A PROSPECTIVE MULTICENTER CASE SERIES. Submitted IN SURGICALLY TREATED DISTAL RADIUS FRACTURES? Submitted 

AFTER DISTAL RADIUS FRACTURE SURGERY

J Orthop Trauma. 2015 Oct;29(10):e414-20.

CHAPTER CHANGES IN DEPRESSION, HEALTH ANXIETY, AND PAIN CATASTROPHIZING BETWEEN ENROLLMENT AND 1 MONTH AFTER A RADIUS FRACTURE

Psychosomatics. 2015 Mar 31. PRESCRIPTION AFTER TREATMENT OF DISTAL RADIUS FRACTURES WITH A VOLAR LOCKING PLATE?

Hand (N Y). 2015 Dec;10(4):639-48. 

Introduction 


\section{INCIDENCE}

Fracture of the distal radius is one of the most frequent upper extremity injuries. If you are a Caucasian woman, the chance you'll sustain a distal forearm fracture is about 1 in 20 by age 80 and 1 in 10 by age 90.' Fractures are somewhat less prevalent among those of African descent and men, perhaps due to a lower incidence of osteoporosis. ${ }^{2}$ The prevalence of distal radius fractures has a bimodal distribution by age: a younger, predominantly male, population sustaining high-energy trauma and an elderly, mainly female, population injured in simple falls from a standing height. ${ }^{3}$ With increasing life expectancy and more active lifestyles among the elderly, the number of distal radius fractures is expected to increase.

\section{UNDERSTANDING THE INJURY}

Most distal radius fractures are caused by a fall on outstretched hand (sometimes referred to by the abbreviated onomatopoeic foosh). Not surprisingly, on icy winter days incidence increases $21 \%$ compared to moderate weather conditions. ${ }^{4}$ Melone identified the basic components involved in a typical intra-articular distal radius fracture and stressed that an unstable malrotated lunate facet fragment benefited from open reduction and internal fixation. ${ }^{5}$ It was also suggested that conceptualizing the wrist in three columns may aid plans for treatment ${ }^{6}$ :

\section{(1) The radial column $=$}

(2) The intermediate column $=$

\section{(3) The ulnar column =}

the scaphoid articular facet, the radial styloid fragment and the radial side of the diaphysis.

$=$ the lunate articular facet, often splits into a volar lunate and dorsal lunate facet fragment, and ulnar side of the diaphysis.

the ulnar head and styloid and the diaphysis of the ulna

An in vivo study suggests that the greatest forces are transmitted across the intermediate column. ${ }^{7}$ With ulnar deviation forces shift to the ulnar side; similarly, with radial deviation forces shift radial (Figure 1).

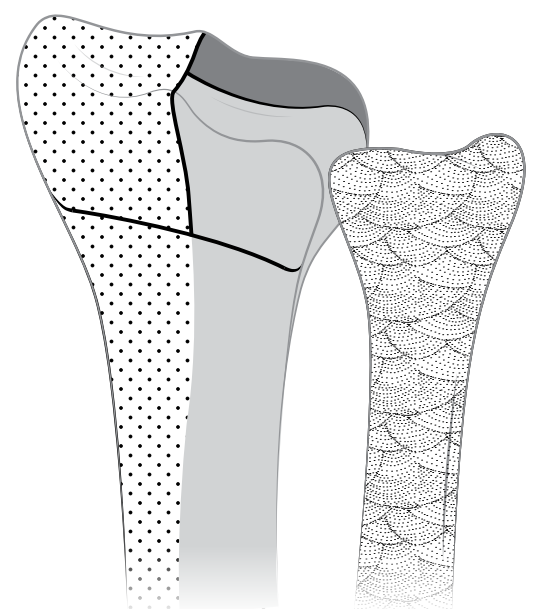

\section{Columns}

$\because$ Radial column

Intermediate column

$\square$ Ulnar column

\section{Fracture fragments}

_ Potential fracture lines

$\because \quad$ Radial styloid fragment

Dorsal lunate facet fragment

Volar lunate facet fragment 
Several eponyms are associated with specific fractures of the distal radius. The most famous is probably named after the Irish surgeon Abraham Colles. He described the external "dinner fork" deformity of the wrist in 1814.8 As Wilhelm Röntgen wasn't born until 1845, the original description includes no radiographic specifications. One of the earliest case-series studying this fracture's radiographs originates from the Massachusetts General Hospital and includes a rudimentary radiographic fracture classification system. ${ }^{9}$

Eponyms make communication convenient, but may leave out important details. Systematic fracture classifications may be more concise. One commonly systematic fracture classification used in research today is the AO/OTA Classification of Fractures and Dislocations ${ }^{10}$ (also recommended by the Dutch distal radius fracture guidelines $\left.{ }^{11}\right)$. This classification consists of three types, A: extra-articular, B: partial intra-articular, with some connection remaining between the diaphysis and metaphysis, and C: complete intra-articular, without such connection. Fractures are further divided into 9 groups (1,2 and 3) and 27 subgroups (.1, .2 and .3). Although popular, this system demonstrates varying levels of agreement, with an inter-rater kappa between 0.37 and 0.68 for fracture types. ${ }^{12,13}$ Reliability further decreases with the incorporation of groups and subgroups. Uncertainty about the classification's reliability is problematic as this impedes its roles to provide a measure of injury severity, provide information for planning treatment, and facilitate scientific communication.

\section{TREATMENT}

Distal radius fractures nearly always heal, but they often heal with deformity. The majority of distal radius fractures are treated non-operatively because either there is little or no deformity or because patients prefer deformity rather than operative treatment. For stable, minimally or non-displaced fractures, treatment with a removable splint and optional follow-up can be offerred. ${ }^{14-16}$

Fractures that are substantially displaced are usually treated with manipulative reduction and splint or cast immobilization. When the reduction does not achieve adequate alignment or the alignment achieved with reduction is not maintained, remanipulation is not effective. The only option for avoiding deformity is surgical stabilization with percutaneous pins, external fixation, or internal fixation. Some studies have attempted to determine probabilities that a fracture will heal with substantial deformity if treated nonoperatively. ${ }^{17,18}$ Patients are increasingly offered operative treatment prior to documented loss of reduction if the probability of healing with deformity is thought to be high with nonoperative treatment. ${ }^{19}$ Also routinely offered surgery are patients with: shearing fractures of the articular margin, fracture-dislocation, and fractures with extensive fragmentation of the articular surface and the metaphysis/diaphysis. ${ }^{18}$

The Dutch distal radius fracture guidelines (based on expert opinion) recommend routine fracture reduction even when surgery is planned. Reduction can relieve soft tissue tension or pressure on the median nerve and might reduce discomfort." However, the risk of soft tissue problems, median neuropathy related to deformity, and increased pain is debatable. Manipulative reduction and splint application also has risks, discomforts, inconveniences, and uses resources.

The correlation between objective impairment and subjective symptoms (e.g. DASH or PRWE scores) is limited. ${ }^{20}$ Following this reasoning, one cannot assume that deformity will cause problems for the patient, 
particularly in low energy injuries in low demand patients. This is illustrated by the fact that at a minimum of 33 years after fracture, no radiographic factors could be related to DASH score in 106 predominantly nonoperatively treated patients. ${ }^{21}$ Also, no radiographic factors, but mainly pain was associated with disability in 84 patients at a minimum of 6 months after fracture. ${ }^{20}$ The reasoning that radiographic deformity doesn't correlate with patient reported outcome measures is counterintuitive to many and additional evidence is needed to change the way surgeons and patients think about treatment and prognosis.

\section{ASPECTS OF RECOVERY}

The traditional biomedical model of illness assumes a direct correspondence between nociception (the pathophysiology of tissue damage, e.g. magnitude of displacement) and pain (the experience of discomfort). There is a growing body of knowledge identifying psychological factors important in determining pain intensity and magnitude of limitations. ${ }^{22,23}$ The biopsychosocial model emphasizes the limited correlation between nociception and pain. It acknowledges the complex interplay of biological, psychological, cultural, and social variables on pain and disability. Surgeons are familiar with some of the psychosocial mediators between nociception and pain, such as secondary gain. They may be less familiar with the influence of symptoms of depression, the tendency to misinterpret or overinterpret nociception (i.e., catastrophic thinking), heightened concern about illness, and social and cultural factors on illness behavior.

Finger stiffness is common during recovery from fracture of the distal radius. When the stiffness, limitations, and pain intensity are considered out of proportion to what is expected, patients are sometimes labeled with illness constructions such as complex regional pain syndrome or reflex sympathetic dystrophy. ${ }^{24}$ The difference between psychological and medical expertise regarding disproportionate pain and disability is striking. Many physicians suspect an as yet elusive pathophysiological process is responsible for the signs and symptoms (biomedical model). Psychologists identify catastrophic thinking, kinesiophobia, and inflexible thinking as the most important factors (biopsychosocial model). If finger stiffness - considered on the continuum on which it occurs - correlates with normal aspects of human illness behavior such as catastrophic thinking on its continuum, we might decide that categorizing patients medically does not facilitate recovery. Instead we might decide to focus on helping patients manage their catastrophic thinking. This could facilitate recovery after distal radius fracture.

Psychological measures such as symptoms of depression, health anxiety, and catastrophic thinking can be quantified using validated questionnaires that have good test-retest reliability in stable individuals. ${ }^{25-27}$ Since they are associated with increased magnitude of disability and pain intensity, interventions targeting psychological measures have been proposed to improve post-surgical outcomes in total knee, hip and shoulder arthorplasty. ${ }^{28-31}$ However, the abrupt loss of function after fracture may influence patients' state of mind differently than slowly progressing osteoarthritis. If psychological measures are to serve as a target for potential improvement in outcome after trauma, we need to know how these constructs behave during recovery.

Greater post-operative opioid consumption correlates with greater symptoms depression, greater catastrophic thinking (preparing for the worst), and greater health anxiety. ${ }^{32,33}$ Opioid centric pain management strategies 
in the United States have created an epidemic of prescription opioid abuse. Most patients have acceptable pain relief with acetaminophen or tramadol after orthopedic surgery in other parts of the world. ${ }^{34-36} \mathrm{Greater}$ opioid consumption does not result in less pain or greater satisfaction with treatment of pain. ${ }^{36}$ Even in the United States where patients are routinely given large opioid prescriptions after surgery for fracture of the distal radius, most patients stop taking opioids within a few days of surgery and take only a few of the pills prescribed. ${ }^{37}$ The current opioid crisis in The United States and Canada was traced to diversion of these unused pills. ${ }^{38}$ Surgeons in the United States and Canada tend to think of the patient that will call complaining of inadequate treatment of their pain and then give more opioids than necessary to the average patient. They also worry about patients running out of opioids, particularly if they live far away, given that opioids cannot be called in to a pharmacy and the patient will need to have a prescription in hand. Knowledge of factors related to a second opioid prescription might inform better pain management protocols and encourage decreased and safer use of opioids after orthopedic surgery in the United States. It might also prevent other countries from moving to an opioid centric pain model.

\section{OUTLINE OF THIS THESIS}

\section{PART 1. UNDERSTANDING THE INJURY}

\section{Chapter 1. Melone's concept revisited: 3D quantification of fragment displacement.}

Melone's concept of intra-articular distal radius fractures - a radial styloid and two lunate facet fragments - is based on wisdom rather than measurements and data. Also Melone felt an unstable malrotated volar lunate facet fragment to be irreducible with manipulation alone and open reduction and plate fixation was recommended.

Quantitative 3D computed tomography (Q3DCT) measures the number, 3D displacement, and articular surface area of fracture fragments. These quantitative measurements might provide a more detailed understanding of fracture morphology.

Important questions addressed

- Can complete articular fractures be divided according to Melone's concept?

- Can Q3DCT be used to accurately assess intra-articular distal radius fracture configuration and displacement? 
Chapter 2. The AO Distal radius fracture classification: global perspective on observer agreement.

The Müller AO classification of distal radius fractures was first published in 1987 as part of the group's overall classification system for long bone fractures. ${ }^{39}$ This scheme was adopted by the Orthopaedic Trauma Association as the system of choice in 2007 and termed the AO/OTA Classification of Fractures and Dislocations. ${ }^{10}$ Also it's the classification recommended by the Dutch distal radius fracture guidelines. ${ }^{\prime \prime}$

Inter- and intra-observer reliability is typically evaluated by having a few surgeons and surgeons-in-training of varying levels of experience evaluate radiographic studies and apply the classification. Because observer variability has an effect on the comparability of various scientific investigations, this might be better assessed by a larger, international cohort.

Important question addressed

- Is the inter-observer reliability similar for AO type A, B and C fractures?

\section{PART 2. TREATMENT}

Chapter 3. No difference in adverse events between surgically treated reduced and unreduced distal radius fractures.

There is a subset of fractures that can be considered for surgery prior to an attempt at manipulative reduction and immobilization, for example due to a marginal shearing injury, significant displacement, or comminution. When surgery is planned before reduction, we typically still reduce the fracture prior to surgery. The rational is to reduce soft tissue tension or pressure on the median nerve, and discomfort. However these issues are debatable and reduction also has risks, discomforts, greater expenses, and inconveniences.

\section{Important question addressed}

- Is closed reduction worthwhile for the subset of patients who choose operative treatment prior to attempted reduction of their distal radius fracture?

Chapter 4. Evaluation of radiographic fracture position one year after variable angle locking volar distal radius plating: a prospective multicenter case series.

The decision to operate on a distal radius fracture is based on the rational that a better aligned articular surface and less displaced fracture improve functional outcome. Effectiveness is therefore also based on maintaining this restored position. It's unknown to what extent reduction is maintained by volar plate fixation as measured by computed tomography (CT).

\section{Important question addressed}

- How well is alignment maintained one year after treatment with a volar locking plate assessed by radiographs and $\mathrm{CT}$ scans?

- Is there a difference in fracture position or functional outcome between one or two distal screw rows? 
Chapter 5. Are radiographic characteristics associated with outcome in surgically treated distal radius fractures?

The association between radiographic deformity and disability is limited. This is illustrated by the difference in parameters recommended by national societies to define an inadequate reduction and consider surgery.

The AAOS (American Academy of Orthopaedic Surgeons) guidelines recommend ${ }^{40}$ :

- more than $3 \mathrm{~mm}$ radial shortening;

- more than $10^{\circ}$ dorsal tilt;

- intra-articular displacement or step-off of more than 2 millimeters.

The Dutch guidelines include" ${ }^{11}$ :

- less then $15^{\circ}$ ulnarward inclination";

- more than $5 \mathrm{~mm}$ shortening;

- more than $15^{\circ}$ dorsal or $20^{\circ}$ volar tilt;

- more than $2 \mathrm{~mm}$ articular displacement;

- joint (sub)luxation.

Both guidelines recommend a strong incorporation of patient preferences due to the limited strength of the recommendations.

The reasoning that radiographic deformity may not correlate with disability is counterintuitive to many and additional evidence is needed to change the way surgeons and patients think about treatment and prognosis.

Important question addressed

- Are radiographic, conventional CT and Q3DCT measures associated with change in change in disability, quality of life, range of motion, and grip strength one year after treatment with a volar locking plate?

Radial inclination is the most commonly used term, but it is inaccurate and an orthopaedic misnomer. The angle being described is the angulation of the articular surface of the radius on the PA view, but otherwise it is not radial. The angle opens ulnarward not radialward. Yet, ulnar inclination is similarly ambiguous. The descriptive term 'ulnarward inclination' is accurate and is increasingly used. 


\section{PART 3. ASPECTS OF RECOVERY}

\section{Chapter 6. Catastrophic thinking is associated with finger stiffness after distal radius fracture surgery.}

Finger stiffness is common after fracture of the distal radius. When the stiffness and pain intensity are considered out of proportion to what is expected, patients are sometimes labeled with illness constructions such as complex regional pain syndrome or reflex sympathetic dystrophy. Constructions that assume some as yet poorly understood underlying pathophysiologic process.

If finger stiffness is considered on the continuum on which it occurs, correlates with normal aspects of human illness behavior such as catastrophic thinking, it might be better to refer to this process descriptively as disproportionate pain and disability. During recovery it might be more helpful the help patients manage their catastrophic thinking, instead of categorizing them as diseased or not.

\section{Important question addressed}

- What factors are associated with finger stiffness at suture removal after volar locking plate fixation, specifically catastrophic thinking (negative beliefs about pain leading to an overprotective response)?

Chapter 7. Changes in depression, health anxiety, and pain catastrophizing between enrollment and 1 month after a radius fracture.

Psychological measures, such as symptoms of depression, health anxiety, and catastrophic thinking, may serve as a target for potential improvement in the outcome after fracture. However, we fist need to know how these constructs behave during recovery.

\section{Important question addressed}

- Is there a difference in symptoms of (1) depression, (2) health anxiety, and (3) catastrophic thinking during the recovery after radius fracture?

Chapter 8. What factors are associated with a second opioid prescription after treatment of distal radius fractures with a volar locking plate?

The United States and Canada are in the midst of an epidemic of opioid addiction and overdose deaths. Most patients in other countries have acceptable pain relief with acetaminophen or tramadol. ${ }^{34-36} \mathrm{Knowledge}$ of the factors associated with a second opioid prescription after volar plate fixation of a fracture of the distal radius in the United States might inform better pain management protocols and encourage decreased and safer use of opioids after volar locking plate fixation.

\section{Important question addressed}

- Are there any differences between patients who do and do not receive a second opioid prescription following treatment of their distal radius fracture with a volar locking plate? 


\section{REFERENCES}

1. Barrett JA, Baron JA, Karagas MR, Beach ML. Fracture risk in the U.S. Medicare population. J Clin Epidemiol. 1999;52(3):243-249.

2. Singer BR, McLauchlan GJ, Robinson CM, Christie J. Epidemiology of fractures in 15,000 adults: the influence of age and gender. J Bone Joint Surg Br. 1998;80(2):243-248.

3. Chen NC, Jupiter JB. Management of distal radial fractures. J Bone Joint Surg Am. 2007;89(9):2051-2062.

4. Giladi AM, Shauver MJ, Ho A, Zhong L, Kim HM, Chung KC. Variation in the incidence of distal radius fractures in the U.S. elderly as related to slippery weather conditions. Plast Reconstr Surg. 2014;133(2):321-332.

5. Melone CP, Jr. Open treatment for displaced articular fractures of the distal radius. Clin Orthop Relat Res. 1986(202):103111.

6. Rikli DA, Regazzoni P. Fractures of the distal end of the radius treated by internal fixation and early function. A preliminary report of 20 cases. J Bone Joint Surg Br. 1996;78(4):588-592.

7. Rikli DA, Honigmann P, Babst R, Cristalli A, Morlock MM, Mittlmeier T. Intra-articular pressure measurement in the radioulnocarpal joint using a novel sensor: in vitro and in vivo results. J Hand Surg Am. 2007;32(1):67-75.

8. Colles A. On the fracture of the carpal extremity of the radius. Edinburgh Medical and Surgical Journal. 1814(10):182186.

9. Codman EA. A study of the x-ray plates of one hundred and forty cases of fracture of the lower end of the radius. The Boston Medical and Surgical Journal. 1900(143):305-308.

10. Marsh JL, Slongo TF, Agel J, et al. Fracture and dislocation classification compendium - 2007: Orthopaedic Trauma Association classification, database and outcomes committee. J Orthop Trauma. 2007;21(10 Suppl):S1-133.

11. Nederlandse Vereniging voor Heelkunde. Richtlijn distale radius fracturen: diagnostiek en behandeling. 2010.

12. Illarramendi A, Gonzalez Della Valle A, Segal E, De Carli P, Maignon G, Gallucci G. Evaluation of simplified Frykman and $\mathrm{AO}$ classifications of fractures of the distal radius. Assessment of interobserver and intraobserver agreement. Int Orthop. 1998;22(2):111-115.

13. Kreder HJ, Hanel DP, McKee M, Jupiter J, McGillivary G, Swiontkowski MF. Consistency of AO fracture classification for the distal radius. J Bone Joint Surg Br. 1996;78(5):726-731.

14. Abbaszadegan H, Conradi P, Jonsson U. Fixation not needed for undisplaced Colles' fracture. Acta Orthop Scand. 1989;60(1):60-62.

15. Dias JJ, Wray CC, Jones JM, Gregg PJ. The value of early mobilisation in the treatment of Colles' fractures. J Bone Joint Surg Br. 1987;69(3):463-467.

16. Finger A, Teunis T, Hageman MG, Thornton E, Neuhaus V, Ring D. Optional follow-up visits for common, low-risk arm fractures. Submitted. 2015.

17. Lafontaine M, Hardy D, Delince P. Stability assessment of distal radius fractures. Injury. 1989;20(4):208-210.

18. Mackenney PJ, McQueen MM, Elton R. Prediction of instability in distal radial fractures. J Bone Joint Surg Am. 2006;88(9): 1944-1951.

19. Chung KC, Shauver MJ, Birkmeyer JD. Trends in the United States in the treatment of distal radial fractures in the elderly. J Bone Joint Surg Am. 2009;91(8):1868-1873.

20. Souer JS, Lozano-Calderon SA, Ring D. Predictors of wrist function and health status after operative treatment of fractures of the distal radius. J Hand Surg Am. 2008;33(2):157-163.

21. Forward DP, Davis TR, Sithole JS. Do young patients with malunited fractures of the distal radius inevitably develop symptomatic post-traumatic osteoarthritis? J Bone Joint Surg Br. 2008;90(5):629-637.

22. Vranceanu AM, Bachoura A, Weening A, Vrahas M, Smith RM, Ring D. Psychological factors predict disability and pain intensity after skeletal trauma. J Bone Joint Surg Am. 2014;96(3):e20.

23. Vranceanu AM, Barsky A, Ring D. Psychosocial aspects of disabling musculoskeletal pain. J Bone Joint Surg Am. 2009;91 (8):2014-2018.

24. Ring D, Barth R, Barsky A. Evidence-based medicine: disproportionate pain and disability. J Hand Surg Am. 2010;35(8):1345-1347.

25. Miller WC, Anton HA, Townson AF. Measurement properties of the CESD scale among individuals with spinal cord injury. Spinal Cord. 2008;46(4):287-292.

26. Stewart SH, Watt MC. Assessment of health anxiety. In: Asmundson GJG, Taylor S, Cox BJ, eds. Health anxiety: Clinical and research perspectives on hypochondriasis and related conditions. New York: Wiley; 2001:95-131.

27. Sullivan MJL, Bishop SR, Pivik J. The Pain Catastrophizing Scale: Development and validation. Psychological Assessment. 1995; 7(4):524-532. 
28. Sullivan M, Tanzer M, Stanish W, et al. Psychological determinants of problematic outcomes following Total Knee Arthroplasty. Pain. 2009;143(1-2):123-129.

29. Wong SE, Zhang AL, Berliner JL, Ma CB, Feeley BT. Preoperative patient-reported scores can predict postoperative outcomes after shoulder arthroplasty. J Shoulder Elbow Surg. 2016.

30. Berliner JL, Brodke DJ, Chan V, SooHoo NF, Bozic KJ. Can Preoperative Patient-reported Outcome Measures Be Used to Predict Meaningful Improvement in Function After TKA? Clin Orthop Relat Res. 2016.

31. Berliner JL, Brodke DJ, Chan V, SooHoo NF, Bozic KJ. John Charnley Award: Preoperative Patient-reported Outcome Measures Predict Clinically Meaningful Improvement in Function After THA. Clin Orthop Relat Res. 2016;474(2):321-329.

32. Helmerhorst GT, Vranceanu AM, Vrahas M, Smith M, Ring D. Risk factors for continued opioid use one to two months after surgery for musculoskeletal trauma. J Bone Joint Surg Am. 2014;96(6):495-499.

33. Ip HY, Abrishami A, Peng PW, Wong J, Chung F. Predictors of postoperative pain and analgesic consumption: a qualitative systematic review. Anesthesiology. 2009;111(3):657-677.

34. Carragee EJ, Vittum D, Truong TP, Burton D. Pain control and cultural norms and expectations after closed femoral shaft fractures. Am J Orthop (Belle Mead NJ). 1999;28(2):97-102.

35. Helmerhorst GT, Lindenhovius AL, Vrahas M, Ring D, Kloen P. Satisfaction with pain relief after operative treatment of an ankle fracture. Injury. 2012;43(11):1958-1961.

36. Lindenhovius AL, Helmerhorst GT, Schnellen AC, Vrahas M, Ring D, Kloen P. Differences in prescription of narcotic pain medication after operative treatment of hip and ankle fractures in the United States and The Netherlands. J Trauma. 2009;67(1):160-164.

37. Rodgers J, Cunningham K, Fitzgerald K, Finnerty E. Opioid consumption following outpatient upper extremity surgery. J Hand Surg Am. 2012;37(4):645-650.

38. Alexander GC, Frattaroli S, Gielen AC. The Prescription Opioid Epidemic: An Evidence-Based Approach. Johns Hopkins Bloomberg School of Public Health. 2015.

39. Müller ME, Nazarian S, Koch P. Classification AO des fractures: les os longs. Berlin: Springer-Verlag; 1987.

40. American Academy of Orthopaedic Surgeons. The treatment of distal radius fractures: guidelines and evidence report. 2009. 
Abbreviations 
3D = three dimensional

AAOS $=$ American Academy of Orthopaedic Surgeons

AO = Arbeitsgemeinschaft für Osteosynthesefragen

AOTK = AO Technical Commissions

ASA classification $=$ American Society for Anesthesiologists classification

CESD $=$ center for epidemiologic studies depression, measures symptoms of depression

$\mathbf{C l}=$ confidence interval

CPT = Current Procedural Terminology

$\mathbf{C T}=$ computed tomography scan

DASH $=$ disabilities of the arm shoulder and hand

EQ5D = EuroQoL5, quality of life measure

FOOSH $=$ onomatopoeic, fall on outstretched hand

ICC = intraclass correlation

ICD = international classification of diseases

IQR = interquartile range

OTA = Orthopaedic Trauma Association

PCS = pain catastrophizing scale

PRWE $=$ patient rated wrist evaluation

Q3DCT = quantitative 3D computed tomography

QuickDASH $=$ short version of the DASH

ROM = range of motion

SD = standard deviation

SE $=$ standard error

VA LCP $=$ variable angle locking plates 


$$
1
$$




\section{Understanding the injury}




\section{CHAPTER}

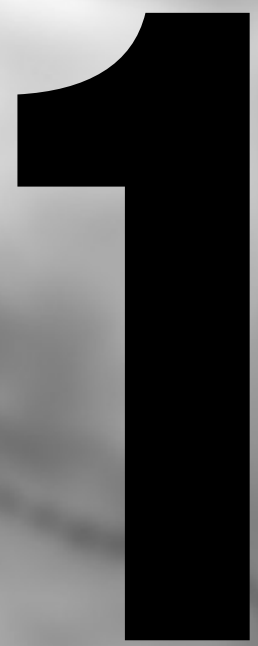




\section{Melone's concept revisited:}

3D quantification of fragment displacement

T. Teunis

N.H. Bosma

B. Lubberts

D.P Ter Meulen

D. Ring 
Importance Half of all distal radius fractures are intra-articular. Melone conceptually divided these fracture into radial styloid and volar and dorsal lunate facet fragments. This classification is useful conceptually to define important facture elements and guide subsequent management, but it's based on wisdom and merits confirmation.

Objectives To determine if complete articular distal radius fractures can be divided according to Melone's concept and if each fragment has similar (1) displacement and (2) articular surface area.

Design Retrospective cohort study.

Setting Urban level 1 trauma center in the United States.

Participants 50 consecutive patients with complete intra-articular (AO type C) distal radius fractures who underwent adequate quality computed tomography scanning between January 5, 2009 and September 24 2012.

Exposure Quantitative 3-dimensional computed tomography.

Main Outcomes Number of fracture fragments, fragment displacement, articular surface, gap deformity and fracture lines.

Results Thirty-eight fractures fit the Melone distribution of fragments. Radial styloid fragments were most displaced, and volar lunate fragments were least displaced. Volar lunate fragments had the largest articular surface area.

Conclusion While these findings confirm Melone's concepts, the finding that volar lunate fragments are relatively large and dorsal lunate fragments relatively small suggests that alignment of the volar lunate fragment with the radial styloid may be the key element of treatment and the dorsal lunate fragment may not routinely benefit from specific reduction and fixation. 


\section{INTRODUCTION}

Approximately half of all distal radius fractures are intra-articular, and the majority (approximately 80\%) are complete articular fractures (AO type C). ${ }^{1,2}$ Melone emphasized the importance of the lunate facet in complete articular type $\mathrm{C}$ fractures based on wisdom rather than measurements and data. In particular he emphasized the importance of a coronal plane fracture line creating separate volar and dorsal lunate facet fragments. An unstable malrotated volar lunate facet fragment was felt to be irreducible with manipulation alone and open reduction and plate fixation was recommended. ${ }^{3-7}$ Medoff divided type $C$ articular fracture fragments into radial column, ulnar corner, dorsal wall, volar rim and free intra-articular fragments. ${ }^{6}$ These classifications are useful conceptually for characterizing the fracture patterns, defining important fracture elements, and directing effective management of each fracture, but they are conceptual and merit confirmation with direct assessment of fracture patterns and measurements.

Quantitative 3D computed tomography (Q3DCT) measures the number, 3D displacement, and articular surface area of fracture fragments. ${ }^{8,9}$ These quantitative measurements might provide a more detailed understanding of fracture morphology, refine our concepts about fracture patterns, and fragment morphology, and help plan treatment ${ }^{9}$. We used these methods to measure fracture fragments of complete intra-articular distal radius fractures to determine if Melone's concepts were accurate and to determine the relative size of the various fragments.

We applied the technique of Q3DCT to complete articular (AO type C) fractures of the distal radius and tested the null hypothesis that $A O$ type $C$ fracture fragments can be divided according to Melone's concepts and that they have similar (1) displacement and (2) articular surface area. Secondarily, we compared gap measured on radiographs and computed tomography scans with Q3DCT gap surface area.

\section{MATERIALS \& METHODS}

Patient selection

After approval by our institutional review board, we retrospectively included 69 consecutive patients with intra-articular distal radius fractures who underwent computed tomography scanning at our institution. Patients were treated between January 5, 2009 and September 24, 2012. We excluded 15 patients with insufficient quality scans (slice thickness $>1.25 \mathrm{~mm}$ ) and 4 patients with $A O$ type B fractures. Fifty patients with $A O$ type $C$ fractures were included in our final analysis. Two authors individually classified the fractures based on 3D models. In case of a discrepancy senior author's opinion was definitive. Median time to computed tomography scan after injury was 1 day (interquartile range/IQR 0-6). All wrists were splinted in the emergency department. Forty fractures (89\%) were reduced prior to computed tomography scanning (177 fracture fragments), 5 were not reduced (19 fragments), and the reduction status could not be determined for 5 fractures (23 fragments). We found no difference in 3D displacement measured by Q3DCT based on reduction status (unreduced $2.8 \mathrm{~mm}$ [IQR 1.2-4.4] vs. reduced $3.4 \mathrm{~mm}$ [IQR 1.7-6.2] vs. unknown $6.1 \mathrm{~mm}$ [IQR 3.4-8.9], $P=0.20)$.

\section{$3 D$ modeling}

Computed tomography scans were saved as Digital Imaging and Communications in Medicine (DICOM) files and were then loaded into 3D Slicer (version 4.2.0 Boston, Massachusetts, United States). A threshold of 250 Hounsfield Units was used to identify fracture fragments; at this threshold even small fragments can 
be identified, but the distortion from surrounding soft tissue is minimal. Subsequently all 3D models were exported into Rhinoceros (Rhinoceros 5.0, McNeel, Seattle, Washington, United States) for further analysis. We determined the number articular fragments, their displacement, articular surface area and articular fracture lines.

The position of the 3D models was standardized using the $x, y$ and $z$ axes in Rhinoceros; the medullary canal was used to define proximal and distal (z-axis), the volar side was used to define radial and ulnar ( $x$-axis), and volar and dorsal (y-axis). All 28 3D models of left-sided radii were mirrored to make the orientation match that of the right-sided radii (see http://www.traumaplatform.org/currentprojects for explanatory video). ${ }^{10}$

\section{Fracture fragments}

Our total of 50 fractures created 180 articular fragments. The majority were classified (by author consensus) as AO type C3 ( $n=41), 6$ as type C2, and 3 as type C1 (Table 1). C3 fractures that could not be classified by Melone and AO type $\mathrm{C} 1$ and C2 are grouped and reported separately. All such fractures consisted of one large fragment and one or more smaller fragments, except for one AO C3.3 fracture that was excluded from analysis.

\section{Displacement}

First, we determined the coordinates for the center of each fracture fragment in 3D space. The fracture was then reassembled using an unfractured distal radius as an overlay template to approximate shape of the native pre-injury anatomy. We defined displacement as the difference between the coordinates of the fracture fragment in this new position and the original coordinates (Figure 1). The overlay template was positioned at the beginning of the study and then remained in this position throughout the study. The template size was adjusted for to match the 3D fracture model, without changing its position. Using the medullary canal of the radial diaphysis we arranged the axes so that displacement on the z-axis represented proximal-distal displacement (loss of height), the x-axis radial-ulnar displacement, and the $y$-axis volar-dorsal displacement. Overall multidirectional (3D-) displacement was defined as combined $x, y$ and $z$ axis displacement:

$$
\text { 3D displacement }(\mathrm{mm})=\sqrt{\Delta x^{2}+\Delta y^{2}+\Delta z^{2}}
$$

In case of a split radial styloid, medial volar or dorsal fragment, the average displacement of the fragments was calculated. 


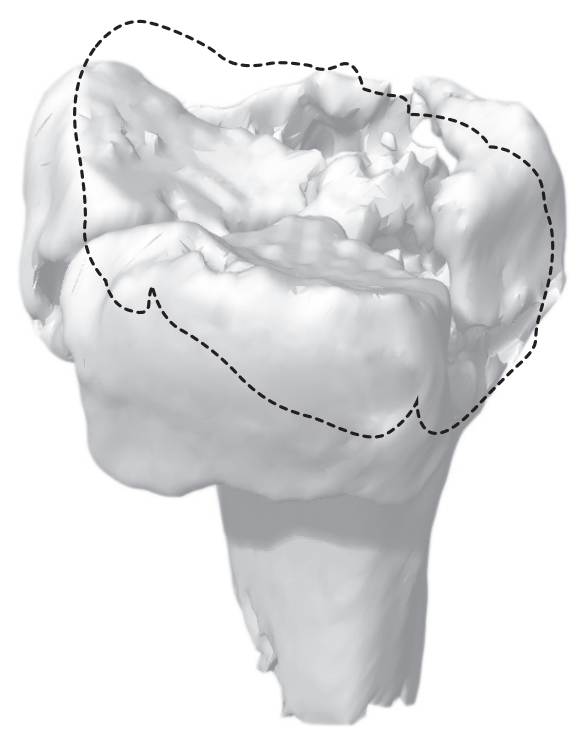

Unfractured distal radius

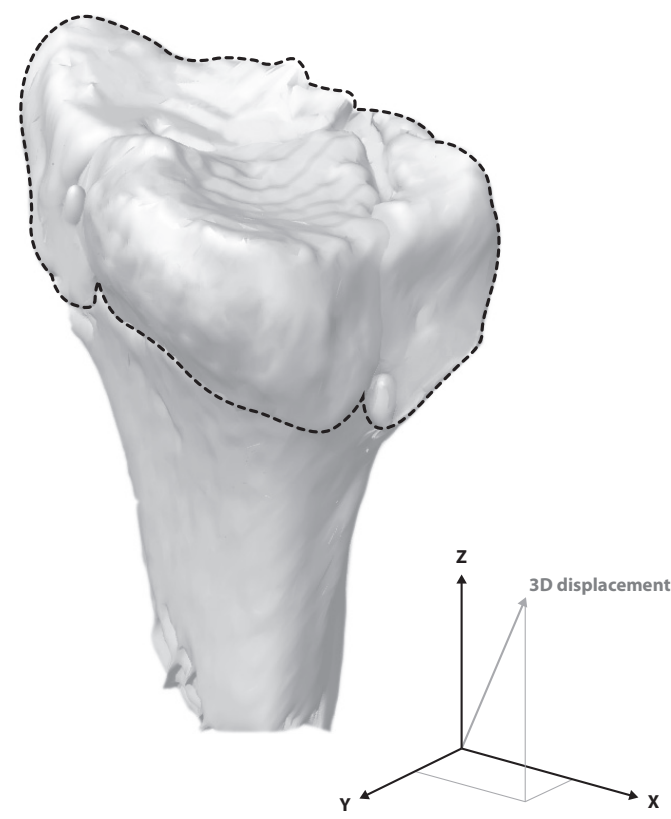

FIGURE 1. (UN)REDUCED DISTAL RADIUS FRACTURE MODEL

A fractured radius is shown on the left, the fractured radial diaphysis is already positioned within that of the template (not shown). The fracture fragments are reduced within the unfractured template, shown on the right. The outline of the template's metaphysis is represented by the red dotted line. In the right lower corner the template's orientation is shown: $z$-axis represents proximal-distal displacement (loss of height), the $x$-axis radial-ulnar displacement, and the $y$-axis volar-dorsal displacement. Overall multidirectional (3D-) displacement is the factor of those axes.

\section{Articular surface \& gap deformity}

Articular surface area was determined by outlining the edges of the articular surface area of each fragment. Subsequently, the surface area, following the contours of the outlined area, was calculated. We compared gap deformity measured on radiographs and in our 3D models. First, we retrieved all radiographs made closest to the time of computed tomography scanning and measured absolute gap deformity on lateral and posterioranterior radiographs as previously described" ' using Onis (2.5 Free Edition, DigitalCore, Tokyo). Secondly, to measure gap deformity between fracture fragments in our 3D models, we outlined the edges of the gap at the level of the articular surface. Subsequently, we measured the size of the gap perpendicular to the $x$-axis (radio-ulnar, similar to posterior-anterior radiographs) and y-axis (volar-dorsal; lateral radiographs) to establish absolute gap deformity. We also calculated the surface area of the outlined gap (Figure 2). 


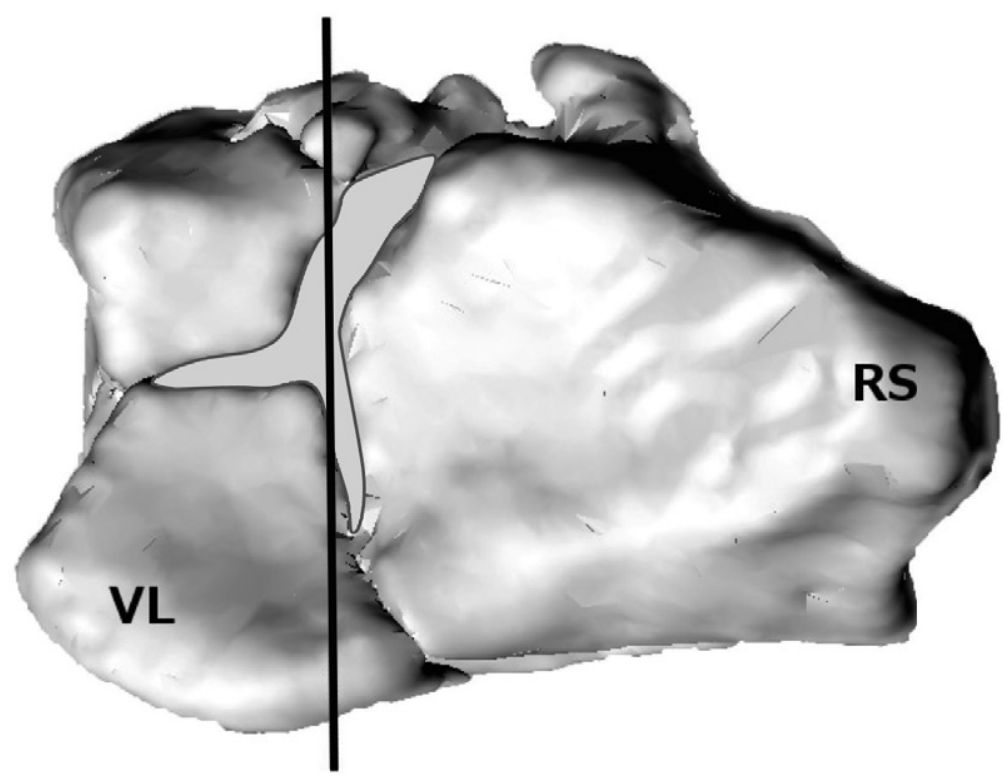

\section{FIGURE 2. OUTLINED AREA OF GAP DEFORMITY}

The top view of the distal radius articular surface is shown. The red line indicates potential measured absolute gap on sagittal CT view, actually representing the split between the lunate facets and radial styloid. This would results in an overestimation of actual gap. Gap surface area is shown in green. RS = radial styloid, $\mathrm{VL}=$ volar lunate facet.

\section{Fracture lines}

To allow for analysis of fracture lines we created similar sized radii by resizing all the fractured radii to match the pre-injury template's size. Subsequently, the carpal articular surface was outlined and each patient's fracture lines were plotted into this outline. Line coordinates were exported to Wolfram Mathematica (Wolfram Mathematica 9.0, Wolfram Research, Champaign, Illinois, United States). We created a heat map by plotting (1) fractures lines that fit the Melone classification and (2) other type C fractures, following light's visible color spectrum: purple indicates a low density and red a high density of fracture lines. The circumference of the model was divided into barren zones and zones with capsular and ligamentous attachment as described by previous studies. ${ }^{12,13}$ We counted the number of fracture line that exited through either type of zone.

\section{Measurement reliability}

Measurement variability can arise in (1) 3D model positioning, (2) fracture reduction, and (3) surface area outlining (see method section displacement and articular surface). The same 2 investigators both independently positioned and measured a set of 10 randomly selected unreduced fracture models to assess the reliability of measuring area and 3D displacement. 


\section{Statistics}

Due to the mainly non Gaussian distribution assessed by Shapiro-Wilk test, we calculated the median and interquartile range and used non-parametric testing. $P$ value less than 0.05 was considered significant. Interobserver agreement of the displacement and articular surface area by the same set of 2 independent observers was evaluated by an intraclass correlation coefficient through a two-way mixed effects model with absolute agreement. Absolute agreement in an intraclass correlation assesses how much each measurement performed per observer differs from the other observer. The intraclass correlation coefficient was 0.82 for 3D displacement ( $95 \%$ confidence interval [CI] 0.69 to 0.90$), 0.91$ for articular surface area $(95 \% \mathrm{Cl} 0.84$ to 0.96$)$ and 0.93 for gap surface $(95 \% \mathrm{Cl} 0.74$ to 0.98$)$, all $\mathrm{P}<0.001$. An intraclass correlation above 0.8 indicates a very high interobserver agreement.

As our sample size was limited by the available computed tomography scans and resources, no power-analysis was performed.

\section{RESULTS}

Of the 41 C3 fractures 38 (93\%) fit the Melone distribution of fragments, but there were 3 exceptions: 1 fracture had a coronal fracture line, but intact sigmoid fossa (AO type C3.3) 2 , 2 had a high coronal/ large die punch fracture that extended more radial with no split in the large volar fragment. Of the 38 fractures that fit the Melone distribution of fragments, 20 fractures had a single radial styloid fragment and 18 were fragmented ( 1 fracture line $n=14,2$ fracture lines $n=3$, and 3 fracture lines $n=1$ ). Thirty-four volar medial fragments were single fragments, and 4 were split into 2 parts. Of the dorsal medial fragments 29 were single fragments, 8 were split once and 1 consisted of 3 parts (Table 1). The majority (62\% [ 118 of 190]) of the fractures lines exited through parts of the radius without ligament attachments (Table 1). A heat map of the Melone fracture patterns shows a relatively intact volar medial fragment (purple), and a high concentration of fractures on the dorsal ridge of the lunate fossa (red) (Figure 3). A heat map of the other fractures indicates a high fracture line density entering the sigmoid fossa.

Among the fractures with typical Melone fragments - that is: a radial styloid and dorsal and volar lunate facet fragment - the radial styloid fragments were on average most displaced, and volar medial fragments were least displaced (Table 1). 


\begin{tabular}{|c|c|c|c|c|}
\hline AO fracture type & Number & & & \\
\hline C1 & 3 & & & \\
\hline C2 & 6 & & & \\
\hline C3 & 41 & & & \\
\hline C3 Melone Fragments & Intact & Split & Double Split & Triple Split \\
\hline Radial Styloid & 20 & 14 & 3 & 1 \\
\hline Volar Medial & 34 & 4 & & \\
\hline Dorsal Medial & 29 & 8 & 1 & \\
\hline C3 Fractures that not fit Melone & Number & & & \\
\hline High Coronal/Die Punch & 2 & & & \\
\hline A0 Type C3.3 & 1 & & & \\
\hline Gap deformity* & Radiographs & CT & Pvalue & \\
\hline Volar-dorsal (y-axis) & $3.8(0-7.0) \mathrm{mm}$ & $11(7.7-17) \mathrm{mm}$ & $<0.001$ & \\
\hline Radio-ulnar (x-axis) & $3.1(0-5.4) \mathrm{mm}$ & $16(8.2-25) \mathrm{mm}$ & $<0.001$ & \\
\hline Fracture lines & Number (\%) & & & \\
\hline Through soft tissue & $72(38 \%)$ & & & \\
\hline Through barren bone & $118(62 \%)$ & & & \\
\hline
\end{tabular}

\section{FIGURE 3. HEAT MAP OF THE DENSITY OF ALL FRACTURE LINES}

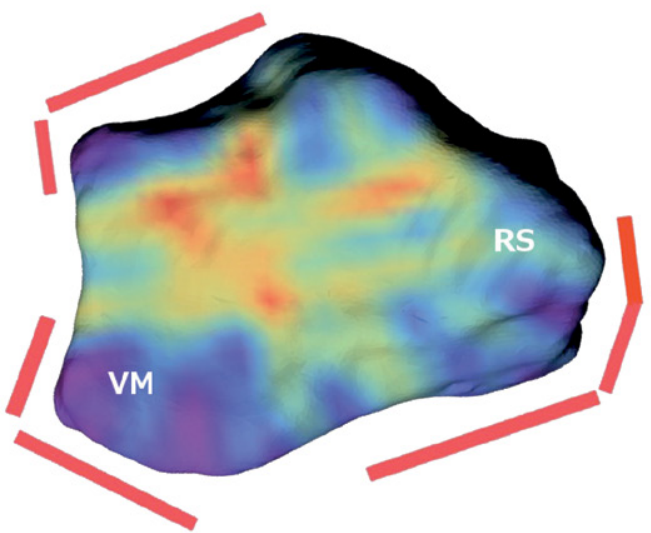

A Melone fractures

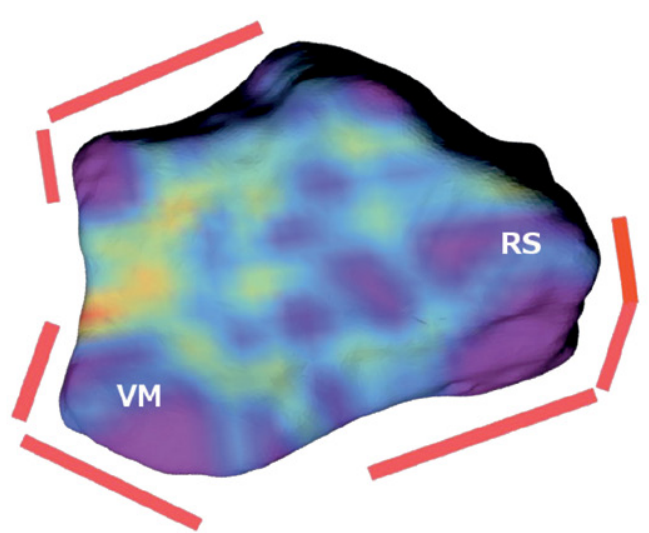

B Other type $C$ fractures

Fracture line density follows light's visible color spectrum: purple indicates a low density

$(<0.5 \%$ of all fracture coordinates) and red a high density ( $>10 \%$ of all fracture coordinates) of fractures.

Red lines indicate ligamentous attachments ${ }^{12,13} . \mathrm{RS}=$ radial styloid, VM = volar medial. 
Volar medial fragments had the largest articular surface area on average (39\% [188 of $\left.484 \mathrm{~mm}^{2}\right]$ ) when compared to radial styloid (37\% [178 of $484 \mathrm{~mm}^{2}$ ) and dorsal medial fragments (24\% [1 $\left.18 \mathrm{of} 484 \mathrm{~mm}^{2}\right]$, $P<0.001$ ) (Table 2). Among type $C$ fractures that did not have the typical Melone fragments, we found no difference in displacement between large and smaller fragments. As one would expect, larger fragments had a larger articular surface (73\% [320 of $439 \mathrm{~mm}^{2}$ ] vs. $27 \%$ [119 of $\left.\left.439 \mathrm{~mm}^{2}\right], P<0.001\right)$.

Table 2. Type C Fractures of the Distal Radius: Displacement and Articular Surface Area

\begin{tabular}{|c|c|c|c|c|c|c|c|c|c|c|c|}
\hline \multirow[b]{2}{*}{$\begin{array}{l}\text { Fractures } \\
\text { fit Melone } \\
\text { classification } \\
(n=38)\end{array}$} & \multicolumn{8}{|c|}{ Displacement of the centroid of the fracture fragment } & \multirow[b]{2}{*}{$\begin{array}{l}\text { Articular } \\
\text { surface }\end{array}$} & \multirow[b]{2}{*}{$P$ value } & \multirow[b]{2}{*}{$\begin{array}{l}\% \text { total } \\
\text { surface }\end{array}$} \\
\hline & 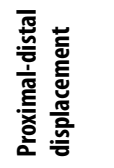 & $\frac{\text { 竞 }}{2}$ & 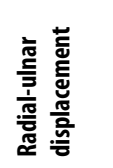 & $\frac{\text { g }}{\mathrm{N}}$ & 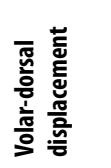 & $\frac{\text { I }}{\mathrm{N}}$ & m. & $\frac{\text { 竞 }}{2}$ & & & \\
\hline Radial Styloid & $\begin{array}{l}2.1 \\
(0.65-5.6)\end{array}$ & & $\begin{array}{l}2.8 \\
(0.87-4.3)\end{array}$ & & $\begin{array}{l}3.1 \\
(1.4-7.4)\end{array}$ & & $\begin{array}{l}5.4 \\
(2.8-10)\end{array}$ & & $\begin{array}{l}149 \\
(91-255)\end{array}$ & & $\begin{array}{l}37 \% \\
(178 / 484)\end{array}$ \\
\hline Volar Medial & $\begin{array}{l}0.97 \\
(0.22-1.9)\end{array}$ & 0.042 & $\begin{array}{l}0.49 \\
(0.03-1.5)\end{array}$ & $<0.001$ & $\begin{array}{l}1.5 \\
(0.3-2.9)\end{array}$ & 0.037 & $\begin{array}{l}2.2 \\
(1.2-4.6)\end{array}$ & 0.0014 & $\begin{array}{l}170 \\
(136-225)\end{array}$ & $<0.001$ & $\begin{array}{l}39 \% \\
(188 / 484)\end{array}$ \\
\hline Dorsal Medial & $\begin{array}{l}1.8 \\
(0.87-3.4)\end{array}$ & & $\begin{array}{l}1.1 \\
(0.32-2.7)\end{array}$ & & $\begin{array}{l}2.5 \\
(0.86-4.7)\end{array}$ & & $\begin{array}{l}3.8 \\
(1.9-6.9)\end{array}$ & & $\begin{array}{l}89 \\
(54-188)\end{array}$ & & $\begin{array}{l}24 \% \\
(118 / 484)\end{array}$ \\
\hline $\begin{array}{l}\text { Other type C } \\
\text { fractures }(n=11)\end{array}$ & & & & & & & & & & & \\
\hline Large fragment & $\begin{array}{l}1.7 \\
(0.43-5.7)\end{array}$ & 036 & $\begin{array}{l}0.55 \\
(0-2.3)\end{array}$ & & $\begin{array}{l}2.5 \\
(0.47-3.4)\end{array}$ & 077 & $\begin{array}{l}6 \\
(2.3-8)\end{array}$ & 0.4 & $\begin{array}{l}274 \\
(252-450)\end{array}$ & & $\begin{array}{l}73 \% \\
(320 / 439)\end{array}$ \\
\hline Smaller fragments & $\begin{array}{l}0.69 \\
(0.34-2.4)\end{array}$ & 0.30 & $\begin{array}{l}1 \\
(0.03-2.5)\end{array}$ & 0.12 & $\begin{array}{l}1.7 \\
(0.01-4.4)\end{array}$ & 0.12 & $\begin{array}{l}2.6 \\
(1.2-5.9)\end{array}$ & 0.41 & $\begin{array}{l}122 \\
(54-141)\end{array}$ & $<0.001$ & $\begin{array}{l}27 \% \\
(119 / 439)\end{array}$ \\
\hline
\end{tabular}

Gap measured on posterior-anterior radiographs did not correlate with gap measured on frontal CT scans ( $\rho=0.26, P=0.093)$. But Q3DCT gap surface area correlated with gap measured on both radiographs $(\rho=0.45, P=0.0023)$ and CT scans $(\rho=0.79, P<0.001)$. Lateral radiographic gap correlated somewhat with sagittal CT gap ( $\rho=0.37, P=0.014)$. Q3DCT gap surface area correlated highly with gap measured both on lateral radiographs $(\rho=0.56, P<0.001)$ and sagittal CT scans $(\rho=0.79, P<0.001)$ (Table 3$)$. The total combined area of the gap between fracture fragments averaged $40 \mathrm{~mm}^{2}\left(21-95 \mathrm{~mm}^{2}\right)$.

\section{Table 3. Correlation of gap deformity measures on radiographs, CT and Q3DCT}

$\begin{array}{llll}\text { Frontal } & \text { Gap surface area } & \text { Radiographs } & \text { Computed tomography } \\ \text { Gap surface area } & \mathrm{x} & \mathrm{x} & \mathrm{x} \\ \text { Radiographs } & 0.45(0.0023) & \mathrm{x} & \mathrm{x} \\ \text { Computed tomography } & 0.79(<0.001) & 0.26(0.093) & \mathrm{X} \\ & & \text { Radiographs } & \mathrm{C} \\ \text { Lateral } & \text { Gap surface area } & \mathrm{x} & \mathrm{x} \\ \text { Gap surface area } & \mathrm{x} & \mathrm{x} & \mathrm{x} \\ \text { Radiographs } & 0.56(<0.001) & 0.37(0.014) & \\ \text { Computed tomography } & 0.79(<0.001) & & \end{array}$




\section{DISCUSSION}

We used quantitative 3D computed tomography (Q3DCT) to measure fracture patterns and fragment characteristics such as displacement in 3 dimensions, articular surface area, and total area of gaps between fragments. We confirmed Melone's concepts about fracture patterns and determined that the volar lunate fragment was much larger than the dorsal lunate fragment on average and the radial styloid fragment had the greatest average displacement.

This study has some limitations. First, the selection of fractures with computed tomography scans and available in our sample may not be representative of the average type $C$ fracture of the distal radius. Most of the fractures in our study featured limited displacement, which is probably representative of type $C$ fractures in general. The amount of displacement is a factor used for surgical decision-making. A study of more displaced fractures using Q3DCT might provide additional information. Secondly, our method does not allow measurement of fragment rotation, which is also an important aspect of fracture morphology. ${ }^{14}$ Thirdly, at least 5 fractures were not reduced prior to computed tomography scanning, which affects the measured displacements and gaps.

Melone's concept is based on wisdom rather than measurements and data. The majority of fractures in our study fit the Melone distribution, confirming his influential ideas. On the other hand, the coronal split in the lunate facet is generally very dorsal creating large volar lunate fragments (Figure 3A) that are more impacted than unstable by the ligamentous attachments, i.e. least displaced (Table 2). Our results also agree with a previous study indicating that fracture lines tend to occur between ligamentous attachments ${ }^{15}$, which may be the reason for the classic Melone grouping of fragments. Previous study found that most of the pressure with axial compression is transmitted across the intermediate column, e.g. the lunate facet. This may be an explanation why articular comminution is found mainly at this level (dorsal and volar lunate facet). Similarly, our heatmap shows the highest density of fracture lines at the intermediate column. ${ }^{16}$

Radial styloid fragments were on average more displaced, and volar lunate fragments were least displaced. Q3DCT measurements of fragment specific 3D displacement might be useful in the study of treatment strategies and outcomes for complete articular fractures of the distal radius. Also, we found that, on average, the volar lunate facet fragment is larger than the dorsal fragment. It's as if type $C$ fractures exit the articular surface rather than the dorsal metaphysis as in a type A fractures. In other words, the dorsal lunate facet may often be part of the dorsal fragmentation typical of a dorsally displaced fracture. If the radiolunate ligaments are intact and the volar lunate facet fragment is reduced and secured, a relatively small, slightly displaced dorsal lunate fragment may not have much affect on function and may not merit an additional dorsal exposure - a hypothesis worth testing.

We encountered the following problem when measuring gap on computed tomography: in a complete articular distal radius fracture with standard Melone configuration there are two fracture lines in the carpal articular surface: (1) running from volar to dorsal with on one side the radial styloid fragment and on the other side the two volar and dorsal medial fragments; and (2) the fracture line between the volar and dorsal medial fragments. Gap measured in the sagittal plane evaluates the distance between the volar and dorsal medial fragments. But when the image is almost exactly within the ventral to dorsal fracture line, this greatly overestimates the actual gap (see red line in Figure 2). The same occurs when assessing gap in frontal plane images: measuring gap within the fracture line between the dorsal and medial fragment, can greatly overestimate this measure. To solve this, we propose Q3DCT surface area measurements for intra-articular 
gaps. By correlating clinical outcome to true surface of these gaps (as opposed to mere one-directional measures) we can gain further understanding of its role in predicting clinical outcome, as some literature suggest the that conventional gap measures do not correlate with functional outcome. ${ }^{17}$

For the creation of 3D models and measurement of fracture characteristics we used free and readily available software. Our results show measurement methods are reproducible between observers. A downside of Q3DCT is the labor intensiveness of 3D model creation, which increases with fracture complexity. Recently, software that uses a computed tomography model from the intact opposite side to facilitate automatic reduction of fracture fragments is now available and might reduce the time per model, but this would require a computed tomography scan of the opposite unfractured wrist. ${ }^{18}$ Further development of this technique might further reduce interobserver variability, allowing Q3DCT to become a more widely used technique for fracture assessment.

Measurement of fracture fragments using software that allows sophisticated imaging and measurement of computed tomography scans can improve our understanding of fractures. We studied a consecutive series of complete articular fractures and found limited displacement and large, easily managed volar lunate facets. The finding that volar ulnar fragments are relatively large and dorsal ulnar fragments relatively small suggests that the highly influential theories and concepts of Melone may only apply to a smaller subset of type C fractures. Combining Q3DCT with patient reported outcome and range of motion might help with the prediction of outcome after distal radius fractures and help guide treatment. Also, the difficulties in treatment of complex articular fractures relate to small volar lunate facets, complex fragmentation, central articular impaction, and more marked displacement. As Q3DCT can accurately quantify these aspects, this method can help to study areas of debate in the treatment of complete articular fractures. For example, (1) what size and displacement of dorsal lunate facet merits a separate dorsal exposure, reduction and fixation? (2) Is there a subset of small volar lunate facet fragments that malrotate and hinders flexion (malangulation of the so-called teardrop on radiographs as described by Medoff)? (3) Is there a subset of small volar lunate facet fragments that can escape fixation and lead to subluxation of the carpus? 


\section{REFERENCES}

1. McQueen MM, Jupiter JB. Radius and ulna. Butterworth-Heinemann; 1999.

2. Müller ME. The Comprehensive classifiction of fractures of long bones. Berlin ;New York: Springer-Verlag; 1990.

3. Browner BD. Skeletal Trauma: Fractures, Dislocations, Ligamentous Injuries. Saunders; 1992.

4. Cooney WP. Fractures of the distal radius. A modern treatment-based classification. Orthop Clin North Am. $1993 ; 24(2): 211-216$

5. Fernandez DL. Fractures of the distal radius: operative treatment. Instr Course Lect. 1993;42:73-88.

6. Medoff RJ. Essential radiographic evaluation for distal radius fractures. Hand Clin. 2005;21(3):279-288.

7. Melone CP, Jr. Distal radius fractures: patterns of articular fragmentation. Orthop Clin North Am. 1993;24(2):239-253.

8. Guitton TG, van der Werf HJ, Ring D. Quantitative measurements of the volume and surface area of the radial head. J Hand Surg Am. 2010;35(3):457-463.

9. Guitton TG, van der Werf HJ, Ring D. Quantitative three-dimensional computed tomography measurement of radial head fractures. J Shoulder Elbow Surg. 2010;19(7):973-977.

10. Teunis T, Bosma NH, Lubberts B, Ter Meulen DP, Ring D. MM Q3DCT Radius. 2014; http://youtu.be/MOniRAoZ2xw Accessed 25 March, 2014.

11. McCallister WV, Smith JM, Knight J, Trumble TE. A cadaver model to evaluate the accuracy and reproducibility of plain radiograph step and gap measurements for intra-articular fracture of the distal radius. J Hand Surg Am. 2004;29(5): $841-847$

12. Berger RA. The ligaments of the wrist. A current overview of anatomy with considerations of their potential functions. Hand Clin. 1997;13(1):63-82.

13. Zumstein MA, Hasan AP, McGuire DT, Eng K, Bain GI. Distal radius attachments of the radiocarpal ligaments: an anatomical study. J Wrist Surg. 2013;2(4):346-350.

14. Melone CP, Jr. Open treatment for displaced articular fractures of the distal radius. Clin Orthop Relat Res. 1986(202): 103-111.

15. Mandziak DG, Watts AC, Bain GI. Ligament contribution to patterns of articular fractures of the distal radius. J Hand Surg Am. $2011 ; 36(10): 1621-1625$

16. Rikli DA, Honigmann P, Babst R, Cristalli A, Morlock MM, Mittlmeier T. Intra-articular pressure measurement in the radioulnocarpal joint using a novel sensor: in vitro and in vivo results. J Hand Surg Am. 2007;32(1):67-75.

17. Catalano LW, 3rd, Cole RJ, Gelberman RH, Evanoff BA, Gilula LA, Borrelli J, Jr. Displaced intra-articular fractures of the distal aspect of the radius. Long-term results in young adults after open reduction and internal fixation. $J$ Bone Joint Surg Am. 1997;79(9):1290-1302.

18. Fritscher K, Karasev P, Pieper S, Kikinis R. Documentation/4.3/Extensions/VirtualFractureReconstruction. 2014; https://www.slicer.org/slicerWiki/index.php/Documentation/4.3/Extensions/VirtualFractureReconstruction. Accessed 25 March, 2014. 
CHAPTER

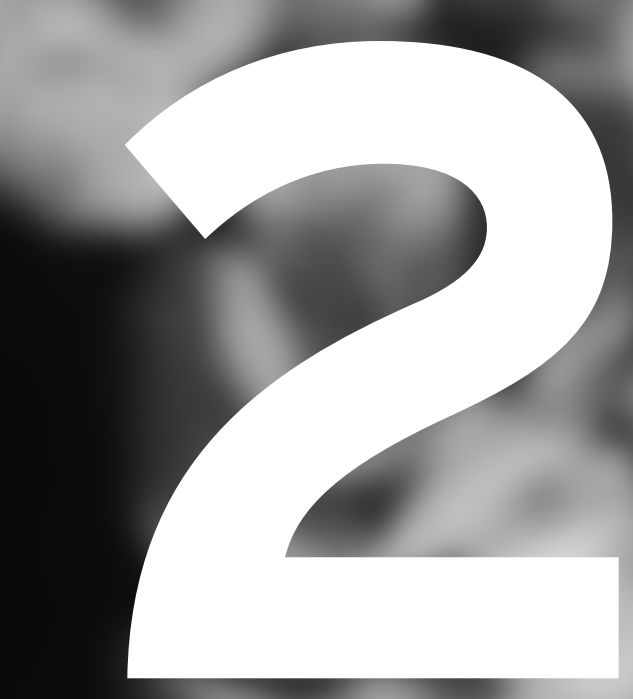




\title{
AO distal radius fracture classification:
}

\section{global perspective}

\section{on observer agreement}

\author{
P. Jayakumar* \\ T. Teunis* \\ B. Bravo Giménez \\ F. Verstreken \\ L. Di Mascio \\ J. Jupiter
}

*Both authors contributed equally

Journal of Wrist Surgery

Poster at the Residents and Fellows Conference \&

Annual Meeting of the American Society for Surgery of the Hand (2015). 
Importance The AO/OTA distal radius fracture classification system is the one of the most widely used classification systems in clinical research today. Observer variability has an effect on the comparability of various scientific investigations.

Objectives To assess inter-observer reliability when classifying fractures by consensus by AO types and groups amongst a large international group of surgeons; secondarily we assessed the difference in inter- and intra-observer agreement of the AO classification in relation to geographical location, level of training and sub-specialty.

Design Reproducibility study.

Setting Single urban medical center.

Participants 96 distal radius fractures for which both CT scans and posterior-anterior and lateral radiographs, rated by 65 observers. Of the observers 10 were female (15\%), 20 practiced in Spain (31\%) and thirteen in the United Kingdom (20\%). 50 were residents or fellows (77\%).

Main Outcomes Inter-observer variability was assessed by intra-class correlation coefficient and intraobserver agreement by Cohen's kappa statistic.

Results We found overall substantial agreement when classifying type A fractures $(0.68,95 \%$ Confidence Interval [Cl] 0.62-74). But agreement was significantly lower for type B (fair agreement, 0.28, 95\% Cl 0.23-0.35) and type $C$ (moderate agreement, $0.44,95 \% \mathrm{Cl} 0.37-0.52$ ) fractures. No difference was observed by location, except for an apparent difference between participants from India and Australia classifying type B fractures (India $0.23,95 \% \mathrm{Cl} 0.16-0.31$ vs. Australia $0.42,95 \% \mathrm{Cl} 0.33-0.53$ ). Although residents had less inter-observer agreement than consultant or attending surgeons for all fracture types, this was not significant. Intra-observer reproducibility was substantial for fracture types $(0.63,95 \% \mathrm{Cl} 0.59-65)$ and only fair for fracture groups (fair $0.40,95 \% \mathrm{Cl} 0.37-0.44)$.

Conclusion Type B classification had the worst agreement, which may reflect the difficulty in categorizing the variety of partial articular fracture configurations. Scientific communication on these particular fracture types might benefit from further information and more consideration e.g. a group of surgeons reaching consensus, rather than a single observer. 


\section{INTRODUCTION}

The Müller AO classification of distal radius fractures was first published in 1987 as part of the group's overall classification system for long bone fractures.' This scheme was adopted by the Orthopaedic Trauma Association as the system of choice in 2007 and termed the AO/OTA Classification of Fractures and Dislocations. ${ }^{2}$ It was originally designed to provide a measure of injury severity, provide information for planning treatment and facilitate scientific communication. ${ }^{3}$ It remains one of the most widely used classification systems for distal radius fractures in clinical research today. The original form classifies fractures into 3 types (A, B and C), 9 groups (1,2 and 3) and 27 subgroups (.1, .2 and .3). ${ }^{3}$ The shortened form including 9 categories (type and group) is most widely used.

This system demonstrates varying levels of agreement, with an inter-observer kappa between 0.37 and 0.68 for fracture types..$^{4,5}$. Inter- and intra-observer agreement of this classification has been typically evaluated by having a few surgeons and surgeons-in-training of varying levels of experience evaluate radiographic studies and apply the classification. Because observer variability has an effect on the comparability of various scientific investigations, evaluation of the system may be improved by using a larger, international cohort. We sought to establish reliability and reproducibility of the AO classification on radiographs and computed tomography scans by a large cohort of observers practicing in different geographical regions. We tested the primary null-hypothesis that inter-observer reliability is similar for AO type A, B and C fractures. Secondarily, we assessed the difference in inter- and intra-observer agreement of the $A O$ classification in relation to geographical location of observers and their level of training and sub-specialty.

\section{MATERIALS \& METHODS}

Study design

After obtaining IRB approval, we included a consecutive series of 96 distal radius fractures, from October 2010 to April 2013, for which both computed tomography scans and posterior-anterior and lateral radiographs were available. The fractures were randomly ordered and images were built into an electronic survey system (REDCap, Vanderbilt University, Tennessee, USA). ${ }^{6}$ At least one computed tomography image through the fracture in the sagittal, coronal and transverse plane along with a 3D view was included, in addition to a lateral and posterior-anterior radiograph. Full consent was acquired for use of the images for research and educational purposes.

We then invited participants to classify all 96 fractures by AO type and group (subgroups were not included). We also provided the option 'AO classification insufficient' for both type and group. Subjects who accepted the invitation and consented to the study were provided a diagrammatic version of the AO classification that could be used during the grading process (Appendix 1). All participants completed the initial survey followed by a second survey independently. Surveys were sent via email link observing a 3-week washout period between each rating. The format included 96 sets of images randomly ordered and then re-ordered for the second survey. Participants were blinded to their previous rating.

\section{Study population}

Participants were recruited from the authors' international network. Acknowledgement, scientific curiosity, and camaraderie were the only incentives for participation. They received the study protocol and, after returning a signed participation form, a link to the first survey. Of the 75 invited, 65 completed the first survey of whom 
50 also classified the fractures a second time. Fifteen percent of the participants were female $(n=10)$.

The majority practiced in Spain $(31 \%, n=20)$ and the United Kingdom $(20 \%, n=13)$. Seventy-seven percent ( $n=50)$ were residents or fellows. Of the consultant or attending level surgeons $(n=15)$, the majority $(n=9)$ were upper extremity specialists (table 1).

\section{Table 1. Observer and fracture demographics}

Variables

Male

Female

Area

- United Kingdom

- Belgium

- The Netherlands

- Spain

- China

- India

- Australia

- Uruguay

Level

- Resident

- Training years

- Attending

- Practice years

Specialty

- Upper Extremity

- Trauma

- General orthopaedics

- Residents

Fractures

Type A

-A.1

- A.2

-A.3

-A.insufficient

Type B

-B.1

-B.2

- B.3

- B.insufficient

Type C

$\cdot C .1$

- $C .2$

$\cdot$. .3

- C.insufficient

A0 type insufficient
$9(14 \%)$

\section{Data}

55 (85\%)

$10(15 \%)$

$13(20 \%)$

$8(12 \%)$

4 (6.2\%)

$20(31 \%)$

$1(1.5 \%)$

$5(7.7 \%)$

$3(4.6 \%)$

$11(17 \%)$

50 (77\%)

$3.1( \pm 2.0)$

15 (23\%)

$10( \pm 8.6)$

$5(7.7 \%)$

$1(1.5 \%)$

$50(77 \%)$

2,933 (27\%)

$52(0.47 \%)$

$1,332(12 \%)$

$1,488(13 \%)$

$61(0.55 \%)$

2,672 (24\%)

587 (5.3\%)

907 (8.2\%)

$1,053(9.5 \%)$

$125(1.1 \%)$

5,200 (47\%)

$1,620(15 \%)$

1,474 (13\%)

1,960 (18\%)

$146(1.3 \%)$

$235(2.1 \%)$

Discrete data presented as number (percentage), continuous data as mean ( \pm standard deviation) 


\section{Statistical Analysis}

To determine the fracture distribution, we averaged all 11,040 ratings (consensus based assessment) (table 1). Inter-observer variability was assessed by intra-class correlation coefficient. This accounted for deflation of inter-observer agreement, which may occur within large cohorts when using multi-rater kappa. Since intra-class correlation coefficient is used for continuous data, we converted all fracture classifications to 0 or 1 scores. For example, an observer rates a fracture $\mathrm{C} 1$. His rating is converted to 1 in the $\mathrm{C} 1$ category and 0 for all other categories. Subsequently the mean intra-class correlation coefficient per fracture and per group and type is calculated. Using this method, there is no golden standard indicating the 'real' fracture type. Instead the intraclass correlation reflects the consensus of the AO fracture type or group of all raters. To calculate the intraclass correlation we used a two-way random effects model for each AO fracture type and group. This model assumes all raters rate the same set of fractures and that they are sampled randomly from a larger population. We report the absolute agreement i.e. how much each measurement performed per observer differed from the other observers.

Intra-observer agreement, the reproducibility of ones previous assessment, was determined by using Cohen's kappa statistic. This measure could only be computed for the 50 assessors who completed both the first and second survey.

The generated values are interpreted according to the guidelines of Landis and Koch. A value of 0.01 to 0.20 indicates slight agreement; 0.21 to 0.40 , fair agreement; 0.41 to 0.60 , moderate agreement; 0.61 to 0.80 , substantial agreement; and 0.81 to 0.99 , almost perfect agreement. Zero indicates no agreement beyond that expected because of chance alone; -1.00 , total disagreement; and +1.00 , perfect agreement. ${ }^{7}$

We regarded non-overlapping confidence intervals as a significant difference; all statistical analyses were conducted using Stata 13.0 (StataCorp LP, Texas, USA).

\section{RESULTS}

By consensus $27 \%(2,933)$ of the fractures were rated as type A, $24 \%(2,672)$ as type B, 47\% $(5,200)$ as type C, and $2 \%(235)$ as unclassifiable (table 1).

We found overall substantial agreement among raters when classifying AO type A fractures $(0.68,95 \%$ Confidence Interval [CI] 0.62-74). But agreement was significantly lower for type B (fair agreement, 0.28, 95\% $\mathrm{Cl}$ 0.23-0.35) and type C (moderate agreement, 0.44, 95\% Cl 0.37-0.52) fractures. We found variable intra-class correlations between $\mathrm{AO}$ fracture group ratings for all three fracture types, with the C.3 classification being most reliably classified (moderate agreement, C.3: 0.45, 95\% Cl 0.39-0.53, vs. slight agreement, C.1: 0.13, 95\% $\mathrm{Cl}$ 0.10-0.17 and C.2: 0.13, 95\% Cl 0.10-0.18).

We found no difference in inter-observer agreement based on practice location when classifying AO type A and type $C$ fractures. Classifying type B fractures, there were no significant differences in inter-observer reliability, except for an apparent difference between India and Australia (India 0.23, 95\% Cl 0.16-0.31 vs. Australia 0.42, 95\% Cl 0.33-0.53).

Although residents had less inter-observer agreement than consultant or attending surgeons for all fracture types, this was not significant. We found no difference in inter-observer reliability between specialty types (table 2). Intra-observer reproducibility was substantial for fracture types $(0.63,95 \% \mathrm{Cl} 0.59-65)$ and only fair for fracture groups (fair, 0.40, 95\% Cl 0.37-0.44). We found no difference in Intra-observer reproducibility based on practice location or level of training (table 3 ). 
Table 2. Intraclass correlation

\begin{tabular}{|c|c|c|c|c|}
\hline Variable & Ratings & Type A & Type B & Type C \\
\hline Overall & 115 & $0.68(0.62-0.74)$ & $0.28(0.23-0.35)$ & $0.44(0.37-0.52)$ \\
\hline \multicolumn{5}{|l|}{ Group } \\
\hline 1 & & $0.026(0.018-0.037)$ & $0.22(0.17-0.28)$ & $0.13(0.10-0.17)$ \\
\hline 2 & & $0.39(0.32-0.46)$ & $0.080(0.061-0.11)$ & $0.13(0.10-0.18)$ \\
\hline 3 & & $0.32(0.27-0.40)$ & $0.31(0.25-0.38)$ & $0.45(0.39-0.53)$ \\
\hline \multicolumn{5}{|l|}{ Level } \\
\hline Residents & 86 & $0.67(0.60-0.73)$ & $0.28(0.23-0.35)$ & $0.42(0.36-0.50)$ \\
\hline Attendings & 29 & $0.73(0.67-0.78)$ & $0.32(0.26-0.40)$ & $0.51(0.44-0.58)$ \\
\hline - General orthopaedics & 2 & $0.66(0.53-0.76)$ & $0.44(0.26-0.58)$ & $0.63(0.49-0.73)$ \\
\hline - Upper Extremity & 17 & $0.72(0.66-0.78)$ & $0.36(0.29-0.44)$ & $0.52(0.45-0.60)$ \\
\hline • Trauma & 10 & $0.74(0.69-0.80)$ & $0.28(0.21-0.37)$ & $0.48(0.40-0.57)$ \\
\hline \multicolumn{5}{|l|}{ Area } \\
\hline United Kingdom & 22 & $0.67(0.61-0.74)$ & $0.31(0.24-0.38)$ & $0.50(0.43-0.58)$ \\
\hline Belgium & 14 & $0.66(0.60-0.73)$ & $0.34(0.27-0.42)$ & $0.47(0.40-0.56)$ \\
\hline The Netherlands & 8 & $0.67(0.59-0.74)$ & $0.35(0.27-0.45)$ & $0.49(0.40-0.58)$ \\
\hline Spain & 38 & $0.70(0.64-0.76)$ & $0.30(0.24-0.37)$ & $0.42(0.35-0.50)$ \\
\hline China & 2 & $0.66(0.53-0.76)$ & $0.44(0.26-0.58)$ & $0.62(0.49-0.73)$ \\
\hline India & 7 & $0.70(0.63-0.76)$ & $0.23(0.16-0.31)$ & $0.37(0.29-0.57)$ \\
\hline Australia & 5 & $0.59(0.49-0.67)$ & $0.42(0.33-0.53)$ & $0.55(0.45-0.64)$ \\
\hline Uruguay & 19 & $0.71(0.65-0.77)$ & $0.27(0.21-0.34)$ & $0.43(0.36-0.51)$ \\
\hline
\end{tabular}

\section{Table 3. Intra-observer reliability}

\begin{tabular}{llll|}
\hline Variable & Raters & Types & Groups \\
Overall & 50 & $0.63(0.59-0.65)$ & $0.40(0.37-0.44)$ \\
Level & & & \\
$\quad$ Residents & 36 & $0.60(0.56-0.64)$ & $0.43(0.36-0.50)$ \\
Attendings & 14 & $0.68(0.63-0.73)$ & $0.39(0.36-0.43)$ \\
$\quad$ General orthopaedics & 1 & 0.59 & 0.28 \\
$\quad$ Upper Extremity & 8 & $0.65(0.58-0.73)$ & $0.49(0.35-0.64)$ \\
$\quad$ Trauma & 5 & $0.73(0.64-0.83)$ & $0.41(0.32-0.50)$ \\
$\quad$ & & & \\
Area & 9 & & $0.38(0.30-0.47)$ \\
United Kingdom & 6 & $0.60(0.51-0.70)$ & $0.36(0.19-0.53)$ \\
Belgium & 4 & $0.59(0.42-1.0)$ & $0.42(0.24-0.59)$ \\
The Netherlands & 18 & $0.71(0.55-0.87)$ & $0.43(0.36-0.49)$ \\
Spain & 1 & $0.63(0.57-0.69)$ & 0.28 \\
China & 2 & 0.59 & $0.40(-0.62-1)$ \\
India & 2 & $0.59(-0.43-1.0)$ & $0.55(-0.21-1)$ \\
Australia & 8 & $0.61(-0.025-1.2)$ & $0.38(0.33-0.44)$ \\
Uruguay & & $0.63(0.58-0.69)$ & \\
Data presented as mean $(95 \%$ confidence interval). & & \\
\hline
\end{tabular}




\section{DISCUSSION}

The classification of fractures by different surgeons at different times needs to be similar and consistent if a system is to become widely utilized. The AO/OTA system is recognized as a useful, inclusive scheme for broad anatomical classification but has been criticized for lack of practical application in surgical decision-making. ${ }^{4,8}$ Previous studies have demonstrated variable inter-observer reliability and intra-observer reproducibility, ranging from fair to substantial (table 4). The majority of these have involved relatively small surgeoninvestigator cohorts. We sought to establish the level of reliability and reproducibility of this classification in a large international cohort of observers of different levels of experience and subspecialty interest. To the best of our knowledge this has not been demonstrated in the literature.

Table 4. Reliability of the AO distal radius fracture classification reported in the literature

\begin{tabular}{|c|c|c|c|c|c|c|c|c|c|c|}
\hline \multirow[b]{2}{*}{ Study } & \multirow[b]{2}{*}{ Modality } & \multirow[b]{2}{*}{ Images } & \multirow[b]{2}{*}{ Raters } & \multirow{2}{*}{$\begin{array}{l}\text { Type of } \\
\text { observers* }\end{array}$} & \multicolumn{3}{|c|}{ Inter-observer reliability (kappa) } & \multicolumn{3}{|c|}{ Intra-observer reliability (kappa) } \\
\hline & & & & & Types & Groups & $\begin{array}{l}\text { Sub- } \\
\text { groups }\end{array}$ & Types & Groups & $\begin{array}{l}\text { Sub- } \\
\text { groups }\end{array}$ \\
\hline Andersen et al., 1996 & radiographs & 55 & 4 & $2 \mathrm{~S}, 2 \mathrm{R}$ & 0.64 & 0.30 & 0.25 & 0.66 & 0.37 & 0.31 \\
\hline Kreder et al., 1996 & radiographs & 30 & 8 & 85 & 0.68 & 0.48 & 0.33 & 0.86 & & \\
\hline $\begin{array}{l}\text { Illarramendi et al., } \\
1998 \text { [1] }\end{array}$ & radiographs & 200 & 6 & $3 \mathrm{~S}, 1 \mathrm{~F}, 2 \mathrm{Rs}$ & 0.37 & & & 0.57 & & \\
\hline \multirow{2}{*}{ Flinkkilä et al., 1998 [2] } & radiographs & \multirow{2}{*}{30} & \multirow{2}{*}{5} & \multirow{2}{*}{$3 S, 2 R$} & 0.48 & 0.23 & 0.18 & & & \\
\hline & $\mathrm{CT}$ & & & & 0.78 & 0.25 & 0.16 & & & \\
\hline McDermid et al., 2001 & radiographs & 67 & 2 & $2 \mathrm{~F}$ & $\begin{array}{l}0.38 \\
(0.15-0.61)\end{array}$ & $\begin{array}{l}0.33 \\
(0.10-0.56)\end{array}$ & & & & \\
\hline Oskam et al., 2001 & radiographs & 124 & 2 & $2 S$ & 0.65 & & & & & \\
\hline \multirow{2}{*}{ Jin et al., 2007 [3] } & \multirow{2}{*}{ radiographs } & \multirow{2}{*}{43} & \multirow{2}{*}{5} & \multirow{2}{*}{$5 S$} & $\begin{array}{l}0.45 \\
(0.31-0.71)\end{array}$ & $\begin{array}{l}0.25 \\
(0.18-0.33)\end{array}$ & & $\begin{array}{l}0.49 \\
(0.45-0.57)\end{array}$ & $\begin{array}{l}0.36 \\
(0.34-0.41)\end{array}$ & \\
\hline & & & & & $\begin{array}{l}0.48 \\
(0.28-0.71)\end{array}$ & $\begin{array}{l}0.29 \\
(0.21-0.71)\end{array}$ & & & & \\
\hline Ploegmakers et al., 2007 & radiographs & 5 & 45 & S\& R, unknown \# & & & & & 0.52 & \\
\hline Belloti et al., 2008 & radiographs & 90 & 5 & $2 S, 1 R, 1 R s, 1 S t$ & & & 0.34 & & & 0.46 \\
\hline Kural et al., 2010 & radiographs & 32 & 9 & 95 & & & 0.096 & & & 0.31 \\
\hline Leerdam et al., 2010 & radiographs & 621 & 2 & $\begin{array}{l}\text { Multiple S vs. } \\
\text { research team }\end{array}$ & 0.60 & 0.41 & 0.33 & & & \\
\hline Küçük et al., 2013 & radiographs & 50 & 20 & $10 \mathrm{~S}, 10 \mathrm{Rs}$ & & & 0.28 & & & 0.44 \\
\hline Siripakarn et al., 2013 & radiographs & 98 & $?$ & 3S, Rs unknown \# & & 0.34 & & & 0.29 & \\
\hline Arealis et al., 2014 & $\begin{array}{l}\text { radiographs } \\
\text { CT }\end{array}$ & 26 & 5 & $5 S$ & & $\begin{array}{l}0.30 \\
0.30\end{array}$ & & & 0.65 & \\
\hline $\begin{array}{l}\text { Prakash et al. } \\
\text { (current study) }\end{array}$ & $\begin{array}{l}\text { radiographs } \\
\& C T\end{array}$ & 96 & 65 & $15 \mathrm{~S}, 50 \mathrm{Rs}$ & & & & $\begin{array}{l}0.63 \\
(0.59-0.65)\end{array}$ & $\begin{array}{l}0.40 \\
(0.37-0.44)\end{array}$ & \\
\hline $\begin{array}{l}1=\text { This study used types } \\
2=\text { This study used } A 0 \text { ty } \\
C T=\text { computed tomograp }\end{array}$ & $\begin{array}{l}\text {, B, C1, C2, C3. } \\
\text { s: } A \& \text { \&, group } \\
y \text {; blank = not }\end{array}$ & $\begin{array}{l}\mathrm{A} 2, \mathrm{~A} 3 \\
\text { neasured }\end{array}$ & $\begin{array}{l}1, C 2, \\
S=\text { sur }\end{array}$ & $\begin{array}{l}3=\text { Inter-obserer } \\
\text { on, } R=\text { radiologist }\end{array}$ & $\begin{array}{l}\text { ured on tw } \\
\text { Fellow, Rs }\end{array}$ & $\begin{array}{l}0 \text { occasions } \\
=\text { Resident }\end{array}$ & studer & & & \\
\hline
\end{tabular}

This study has some limitations. Firstly, there were no requirements placed on the types of distal radius fractures acquired for the study which led to extremely low numbers of certain configurations e.g. type $\mathrm{Al}$ fractures $(0.3 \%$ by consensus based assessment). Although this is not a limitation in itself, when only a few observers choose a specific outcome, intra-class correlation loses reliability and thus, the intra-class correlation for Al fractures is probably not reliably assessed. Limited numbers were also observed in subspecialty with 
only one general orthopaedic surgeon included limiting the strength of the comparison with this sub-group. Secondly, selection bias may exist given that all these injuries underwent CT scanning, suggesting a tendency for selecting more complex fracture configurations that may have warranted more detailed imaging. Thus, the range of images tested may not be an accurate representation of all fracture types. Moreover, the CT images themselves were also specific screenshots and observers were unable to gain control and scroll through the series, which may have influenced the classification. Thirdly, some of the radiographs consisted of fractures in plaster. Although the classification does not specify which images to use when rating these injuries, reduced fractures may prevent a complete appreciation of the original configuration.

Fourth, regarding our guidelines for assessing kappa, we recognized that the interpretation by Fleiss et al., is less favorable than that of Landis \& Koch. However, both set ranges somewhat arbitrarily and we selected the latter given it appeared to be the most widely quoted, utilized in almost all of our identified series of studies and included a higher number of grades, potentially providing greater depth to the conclusions. Fifth, participants were informed this was a test-retest investigation and this may in itself have created some bias in intra-observer variability. This was difficult to avoid due to logistics of participant recruitment. Finally, the process of recruiting observers willing to participate in this study may be open to selection bias. However, we aimed to limit this by performing the investigation in a relatively large group of participants. We found different levels of agreement among raters when classifying AO fracture types and groups. Previous studies don't distinguish between different AO fracture types, groups, and subgroups, and use kappa statistics; this makes it difficult to compare our study to prior results. Kappa for inter-observer reliability from studies assessing $\mathrm{AO}$ fracture types ranges from 0.37 to $0.78^{4,9}$; between groups it ranges from 0.23 to $0.48^{5,9}$; and between subgroups from 0.094 to $0.34 .^{10,11}$ The varying results might be explained by difference in case mix (more AO type B fractures would results in lower agreement) and the different number of observers, ranging between two and eight for types and groups, and two and twenty for subgroups (table 4). Higher levels of inter-observer agreement with type A fractures may not be surprising as one assumes that the distinction between extra- and intra-articular fractures may be clearer to define than partial and complete articular configurations. Making a clear distinction between a fracture involving part of a joint surface versus one with simple articular involvement and unclear diaphyseal separation (C.1 fractures) may be more challenging. The type B classification, in particular, had the worst agreement in our study and this may reflect the difficulty in categorizing the variety of partial articular fracture configurations, such as 'die-punch' fractures. Communication on these particular fracture types might benefit from further information and more consideration.

From the geographical perspective, observers had similar agreement classifying type $A$ and $C$ fractures, but observers from India had less inter-observer reliability than observers from Australia when classifying type $B$ fractures. No previous study compares difference in inter-observer reliability based on geographic location. Our results again seem to emphasize the difficulty classifying AO type B fractures. This is of particular interest to the international scientific community reporting on these fracture types. Future research could assess how to more reliably classify partial intra-articular fractures.

We found no difference in inter-observer reliability between specialty types. Although residents had less interobserver agreement than consultant or attending surgeons for all fracture types, this was not significant. It could be assumed that the level of agreement amongst residents may not be as high as the more senior and experienced surgeons, despite a lack of statistical significance. On the other hand, previous study also found 
low but similar correlation for inter-observer reliability between observers depending on experience for $\mathrm{AO}$ groups (spearman correlation $<6$ years experience: $0.10, P=0.04$ vs. $\geq 6$ years experience: $0.10, P=0.05$; total 45 observers, unknown distribution between groups). ${ }^{12}$ Another study also found similar inter-observer reliability between ten residents and ten surgeons for AO subgroups (mean kappa residents: 0.30 vs. surgeons 0.32). ${ }^{13}$ These results and ours suggest that the $A O$ classification scheme can reliably be used by trainees and more experienced users, and between specialties in terms of inter-observer reliability.

In our study kappa intra-observer reproducibility was substantial $(0.63,95 \% \mathrm{Cl} 0.59-65)$ for fracture types and fair for groups $(0.40,95 \% \mathrm{Cl} 0.37-0.44)$. In the literature kappa for AO type ranges between 0.49 and $0.66^{14,15}$ between 0.29 and 0.65 for groups ${ }^{16,17}$; and between 0.31 and 0.46 for subgroups (table 4 ). ${ }^{10,11,15}$ We found no difference in intra-observer reproducibility based on practice location or level of training. Prior results vary for intra-observer reliability by experience. One previous study found higher intra-observer agreement between younger raters ( 1 fellow and 2 residents) than 3 orthopaedic surgeons (kappa 0.63 trainees vs. 0.50 surgeons, $P<0.03)^{4}$. Another study found higher intra-observer reliability of subgroups between 10 residents (kappa 0.37) and 10 surgeons (kappa 0.50). ${ }^{13}$ Similar to a study by Andersen et al. we found no difference in intra-observer reliability by specialty. They found similar kappa values between 2 radiologists and 2 hand surgeons. ${ }^{15}$ The AO classification seems reproducible by observers, but reliability decreases as groups and subgroups are included. It's unclear if there is a difference in intra-observer reliability based on experience. This is something future study could assess.

We established reliability of AO type and group classification in a large, international cohort. Inter-observer reliability varies between types and groups, and between practice locations. Type B, C.1 and C.2 fractures had the worst inter-observer reliability. Communication on these particular fracture types might benefit from further information and more consideration. This study highlights these challenging fracture types and provides a global perspective on the utilization of this system.

\section{ACKNOWLEDGEMENTS}

We thank Dr Alberto Fernandez MD for the images and scientific advice, The Harvard Catalyst for their statistical support and clinical investigators who were integral to data collection: Acosta Zaro A, Allen L, Al Hakim W, Andres H, Angel A, Arroyo M, Auplish S, Belloni A, Bernat A, Bertoni J, Brown K, Canizares A, Capel Agundez A, Carvajal R, Cecilia Lopez D, Cesar Cordova Peralta J, Clitherow H, Clockaerts S, Crosa F, Curion N, De Keyzer P, Devendra A, Dheep K, Dirckx M, Fei W, Fleming S, Frima H, Gallardo J, Garcia C, Garcia L, Garcia de la Fuente P, Garcia Fernandez D, Gonzalez C, Gordon M, Hernandez Rath D, Hollman F, Houwert M, Jeyaseelan L, Jiminez V, Kahane S, Lasa A, Lockey J, Martinez Leocadio M, Martin Fuentes A, Mathew P, Middleton C, Minnen L, Mulligan A, Munoz M, Olayo, Paramo P, Quintana Plaza J, Rashid M, Robledo H, Ross M, Srikanth K, Stoffelen D, Taylor M, Vacas E, Van Gestel L, Vanhees M, Vanhoecke E, Verstreken F, Zorilla Sanchez de Neyra J. 


\section{REFERENCES}

1. Müller ME, Nazarian S, Koch P. Classification AO des fractures: les os longs. Berlin: Springer-Verlag; 1987.

2. Marsh JL, Slongo TF, Agel J, et al. Fracture and dislocation classification compendium - 2007: Orthopaedic Trauma Association classification, database and outcomes committee. J Orthop Trauma. 2007;21(10 Suppl):S1-133.

3. Müller ME. The Comprehensive classifiction of fractures of long bones. Berlin ;New York: Springer-Verlag; 1990.

4. Illarramendi A, Gonzalez Della Valle A, Segal E, De Carli P, Maignon G, Gallucci G. Evaluation of simplified Frykman and $\mathrm{AO}$ classifications of fractures of the distal radius. Assessment of interobserver and intraobserver agreement. Int Orthop. 1998;22(2):111-115.

5. Kreder HJ, Hanel DP, McKee M, Jupiter J, McGillivary G, Swiontkowski MF. Consistency of AO fracture classification for the distal radius. J Bone Joint Surg Br. 1996;78(5):726-731

6. Harris PA, Taylor R, Thielke R, Payne J, Gonzalez N, Conde JG. Research electronic data capture (REDCap)--a metadatadriven methodology and workflow process for providing translational research informatics support. J Biomed Inform. 2009;42(2):377-381.

7. Landis JR, Koch GG. The measurement of observer agreement for categorical data. Biometrics. 1977;33(1):159-174.

8. Trumble TE, Culp R, Hanel HP, Geissler WB, Berger RA. Instructional Course Lectures, The American Academy of Orthopaedic Surgeons - Intra-Articular Fractures of the Distal Aspect of the Radius. J Bone Joint Surg Am. 1998;80(4):582600 .

9. Flikkila T, Nikkola-Sihto A, Kaarela O, Paakko E, Raatikainen T. Poor interobserver reliability of AO classification of fractures of the distal radius. Additional computed tomography is of minor value. J Bone Joint Surg Br. 1998;80(4):670 672.

10. Kural C, Sungur I, Kaya I, Ugras A, Erturk A, Cetinus E. Evaluation of the reliability of classification systems used for distal radius fractures. Orthopedics. 2010;33(11):801.

11. Belloti JC, Tamaoki MJ, Franciozi CE, et al. Are distal radius fracture classifications reproducible? Intra and interobserver agreement. Sao Paulo Med J. 2008;126(3):180-185.

12. Ploegmakers JJ, Mader K, Pennig D, Verheyen CC. Four distal radial fracture classification systems tested amongst a large panel of Dutch trauma surgeons. Injury. 2007;38(11):1268-1272.

13. Kucuk L, Kumbaraci M, Gunay H, Karapinar L, Ozdemir O. Reliability and reproducibility of classifications for distal radius fractures. Acta Orthop Traumatol Turc. 2013;47(3):153-157.

14. Jin WJ, Jiang LS, Shen L, et al. The interobserver and intraobserver reliability of the cooney classification of distal radius fractures between experienced orthopaedic surgeons. J Hand Surg Eur Vol. 2007;32(5):509-51 1.

15. Andersen DJ, Blair WF, Steyers CM, Jr., Adams BD, el-Khouri GY, Brandser EA. Classification of distal radius fractures: an analysis of interobserver reliability and intraobserver reproducibility. J Hand Surg Am. 1996;21 (4):574-582.

16. Siripakarn Y, Niempoog S, Boontanapibul K. The comparative study of reliability and reproducibility of distal radius' fracture classification among: AO frykman and Fernandez classification systems. J Med Assoc Thai. 2013;96(1):52-57.

17. Arealis G, Galanopoulos I, Nikolaou VS, Lacon A, Ashwood N, Kitsis C. Does the CT improve inter- and intraobserver agreement for the AO, Fernandez and Universal classification systems for distal radius fractures? Injury. 2014;45(10):1579-1584.

18. Jupiter JB, Fernandez DL. Comparative classification for fractures of the distal end of the radius. J Hand Surg Am. 1997;22(4):563-571 
PART

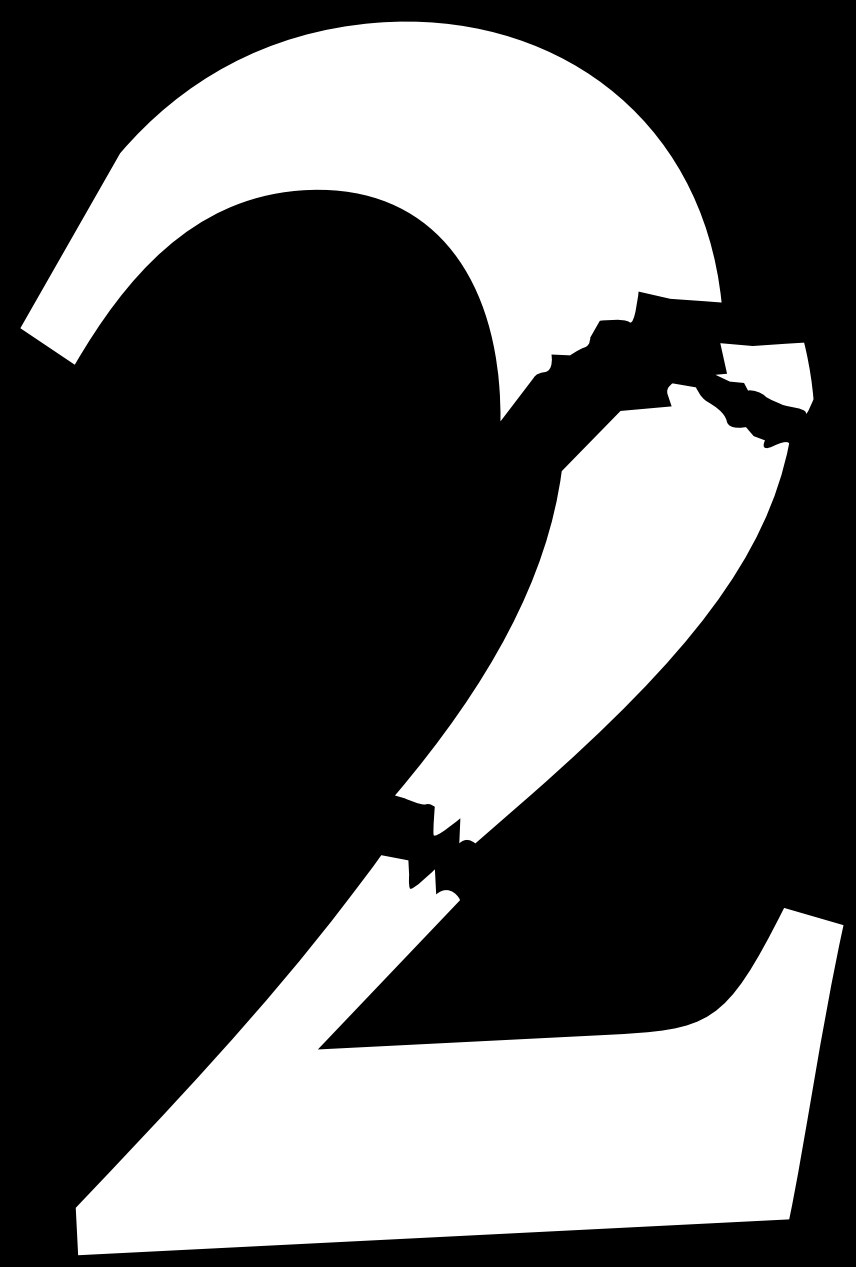




\section{Treatment}


CHAPTER

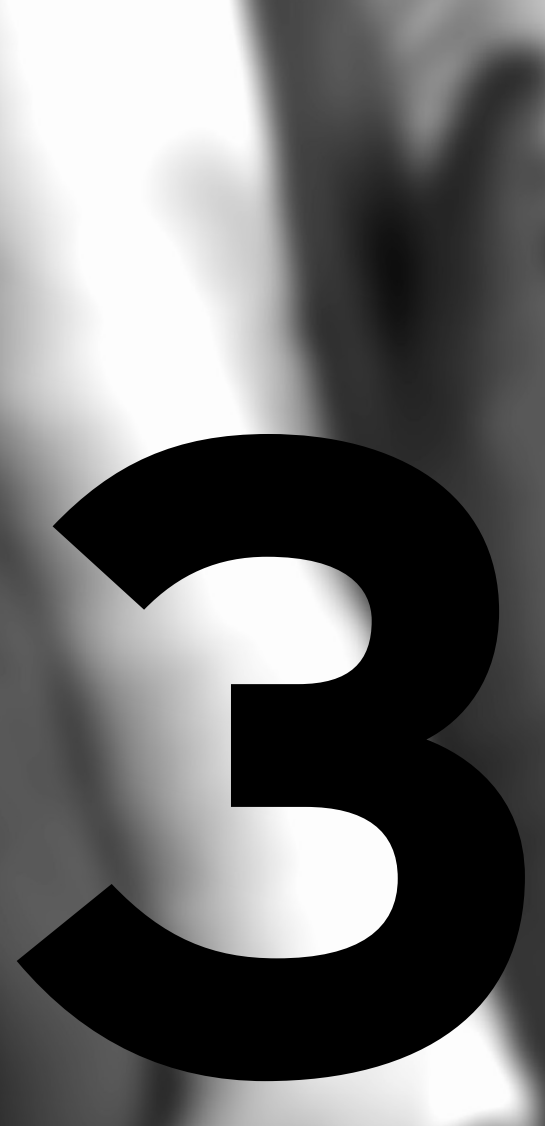




\section{No difference in adverse events}

between surgically treated

reduced and unreduced distal

radius fractures

T. Teunis

F. Mulder

S.P. Nota

L.W. Milne

G.S. Dyer

D. Ring

J Orthop Trauma. 2015 Nov;29(1 1):521-5.

Presented at the European Congress of Trauma \& Emergency Surgery (2015), Annual Meeting of the American Academy of Orthopaedic Surgeons (2015), and the Annual Harvard Orthopedic Trauma Research Day (2015).

Selected stop at the poster tour session at the Annual Meeting of the Orthopaedic Trauma Association (2014). 
Importance Most distal radius fractures are not considered for surgery until manipulative reduction is attempted. There is also a subset of fractures that can be considered for surgery prior to an attempt at manipulative reduction due to significant displacement or comminution.

Objectives To determine if closed reduction is worthwhile for the subset of patients who choose operative treatment before attempted reduction of their distal radius fracture. We hypothesize that there are no differences in (1) adverse events and (2) subsequent surgeries between patients treated with manipulative reduction compared with those that were splinted without reduction.

Design Retrospective cohort study.

Setting Three affiliated urban hospitals in a single city in the United States.

Participants 1511 consecutive adult patients who underwent open reduction and internal fixation of their distal radius fracture between January 1, 2007, and December 31, 2012, of whom 102 (7\%) were not reduced before surgery.

Exposure Manipulative reduction compared with splinting without reduction.

Main Outcomes Adverse events were defined as any infections, hematomas treated operatively, disproportionate finger stiffness, (transient) neuropathology after surgery, delayed carpal tunnel release, malunion, reoperation for loss of alignment, hardware removal, and tendon ruptures within 1 year after surgery. Outcome measures were grouped to determine the overall adverse event rate and subsequent surgery rate.

Results We found no difference in specific adverse events between unreduced and reduced fractures. After adjusting for possible confounding variables by logistic regression, we found no difference in overall rates of adverse events (adjusted odds ratio unreduced fractures 1.2, 95\% confidence interval 0.67-2.0) and subsequent surgeries (adjusted odds ratio unreduced fractures $0.65,95 \%$ confidence interval 0.23-1.8).

Conclusion Leaving the fracture unreduced before surgery was not associated with increased adverse events or subsequent surgeries. For patients who make an informed decision to undergo operative treatment for their closed neurovascular intact displaced distal radius fracture, manipulative reduction may not be helpful.

Level of Evidence Therapeutic level III 


\section{INTRODUCTION}

Most distal radius fractures are not considered for surgery until manipulative reduction is attempted. When the reduction does not achieve adequate alignment or the alignment achieved with reduction is lost, a fracture is considered unstable and might be considered for surgery.

Fractures with compartment syndrome, median nerve dysfunction, an open wound, or pressure on the skin from a displaced fracture fragment are treated with prompt surgery. There is also a subset of fractures that can be considered for surgery prior to an attempt at manipulative reduction and immobilization. Examples include fractures with significant displacement or comminution', fractures that are part of a more complex arm injury (e.g. other fractures, dislocations, wounds, or burns), and bilateral injuries. ${ }^{2}$ When surgery is planned before reduction, we typically reduce the fracture prior to surgery. Reduction can relieve soft tissue tension or pressure on the median nerve and might reduce discomfort. However, the risk of soft tissue problems, median neuropathy related to deformity, and increase pain is debatable. Manipulative reduction and splint application also has risks, discomforts, greater expenses, and inconveniences.

The aim of our study was to assess the balance between risks and benefits of closed reduction in the subset of patients who choose operative treatment before attempted reduction. We hypothesize that there are no differences in (1) adverse events and (2) subsequent surgeries between patients treated with manipulative reduction compared to those that were splinted without reduction prior to distal radius fracture surgery. As complications might imply that there were technical errors, or that something might have been done better, we prefer the term adverse events. This term is inclusive of problems that arise due to the disease, the treatment, or inadvertently.

\section{PATIENTS \& METHODS}

Patient selection

After approval by our institutional review board, we queried the research database of 3 affiliated urban hospitals in a single city in the United States for eligible patients. All adult (age 18 or greater) patients who underwent open reduction and internal fixation of their distal radius fracture identified by Current Procedural Terminology (CPT) code, i.e. 25607, 25608 and 25609, between January $1^{\text {st }} 2007$ and December $31^{\text {st }} 2012$ were retrospectively included. Data was retrieved through December $31^{\text {st }}, 2013$ resulting in a minimum 1-year follow-up. Of 3 patients with bilateral fractures treated operatively, both sides were included separately. This resulted in an initial cohort of 1771 patients.

Pathological $(n=1)$, open $(n=134)$, patients with skin tenting $(n=4)$, neuropathy $(n=57)$, or compartment syndrome $(n=3)$ were excluded. One patient who attempted reduction himself prior to visiting the Emergency Department was excluded and 1 patient with a second fracture at the same location within 1 year.

\section{Patient population}

After excluding 59 fractures in which the status of reduction could not be determined from the physician's notes, there were 1511 patients available for analysis of whom 102 (6.8\%) fractures were not reduced prior to surgery. Reductions were performed using hematoma blocks. The decision to proceed to surgery was made by the patient and surgeon together based on the expected instability as estimated by the treating surgeon. Open reduction and internal fixation was performed an average of 6 days after first presentation to our Emergency Department or outpatient clinic. There was no difference between reduced ( $6 \pm 5$, range $0-46$ 
days) and unreduced ( $5 \pm 5$, range 0-21 days) fractures $(P=0.092)$. There were more women among unreduced patients (68\% [958 of 1409] reduced vs. $80 \%$ [ 82 of 102] unreduced, $P=0.008)$. Unreduced patients more often presented to an outside Emergency Department (reduced 43\% [610 of 1409] vs. unreduced 86\% [88 of 102], $P=<0.001$ ). We found no difference in age, fracture type (extra-articular, intra-articular with 2 or more than 2 articular fragments; fracture type was identified by CPT code), type of surgery (plating vs. other), or carpal tunnel release at the time of index surgery between reduced and unreduced fracture groups (Table 1). Sixtyfour surgeons, of whom 12 performed more than 40 surgeries, treated our cohort.

To specifically compare baseline pre-reduction radiographic parameters we matched all available $(n=71)$ radiographs from patients that did not have reduction $1: 1$ to a patient that did have reduction by age, sex and fracture type (Command optmatch2, Stata 13, StataCorp LP, College Station, Texas). We measured (1) ulnarward inclination; (2) ulnar variance; (3) volar tilt; (4) dorsal translation; and (5) ulna intact. ${ }^{3}$ Two investigators measured and checked the parameters together (TT \& SPN, research fellows), blinded for fracture manipulation status. Discordant judgment was resolved by the senior author.

Unreduced fractures had more ulnarward inclination (reduced $10^{\circ} \pm 8.5^{\circ}$ vs. unreduced $14^{\circ} \pm 7.6^{\circ}, P=0.0051$ ) . Other radiographic parameters did not differ between fractures (Table 1).

\section{Table 1. Baseline characteristics of operated reduced and unreduced distal radius fractures}

\begin{tabular}{|c|c|c|c|c|}
\hline Patient characteristics & Total & Reduced & Unreduced & P value \\
\hline Fractures & 1511 & 1409 & 102 & \\
\hline Age & $54( \pm 16)$ & $54( \pm 16)$ & $54( \pm 17)$ & 0.78 \\
\hline Female & $69 \%(1040)$ & $68 \%(958)$ & $80 \%(82)$ & 0.008 \\
\hline \multicolumn{5}{|l|}{ Fracture type } \\
\hline Extra-articular fracture & $29 \%(435)$ & $29 \%(407)$ & $27 \%(28)$ & \\
\hline Intra-articular, 2 fragments & $26 \%(389)$ & $26 \%(366)$ & $23 \%(23)$ & 0.63 \\
\hline Intra-articular, $\geq 3$ fragments & $45 \%(687)$ & $45 \%(636)$ & $50 \%(51)$ & \\
\hline \multicolumn{5}{|l|}{ Treatment characteristics } \\
\hline First presentation at outside Emergency Department & $46 \%(698)$ & $43 \%(610)$ & $86 \%(88)$ & $<0.001$ \\
\hline Days between first presentation and surgery & $5.8( \pm 5.1)$ & $5.9( \pm 5.2)$ & $5.0( \pm 4.6)$ & 0.092 \\
\hline Treatment other than volar locking plate & $4.0 \%(61)$ & $4.1 \%(57)$ & $3.9 \%(4)$ & 1.0 \\
\hline Concomittant carpal tunnel release & $11 \%(168)$ & $11 \%(156)$ & $12 \%(12)$ & 0.87 \\
\hline \multicolumn{5}{|l|}{ Matched radiographic parameters* } \\
\hline Ulnarward inclincation & $12^{\circ}\left( \pm 8.2^{\circ}\right)$ & $10^{\circ}\left( \pm 8.5^{\circ}\right)$ & $14^{\circ}\left( \pm 7.6^{\circ}\right)$ & 0.005 \\
\hline Ulnar variance $(\mathrm{mm})$ & $1.7( \pm 5.0)$ & $2.3( \pm 6.1)$ & $1.2( \pm 3.5)$ & 0.19 \\
\hline Volar tilt & $-9.4^{\circ}\left( \pm 21^{\circ}\right)$ & $-10^{\circ}\left( \pm 23^{\circ}\right)$ & $-8.7^{\circ}\left( \pm 20^{\circ}\right)$ & 0.72 \\
\hline Dorsal translation (mm) & $-15( \pm 17)$ & $-15( \pm 20)$ & $-12( \pm 14)$ & 0.32 \\
\hline Ulna intact & $53 \%(75)$ & $48 \%(34)$ & $58 \%(41)$ & 0.73 \\
\hline
\end{tabular}




\section{Outcome measures}

Through chart review, CPT and International Classification of Diseases (ICD)-9 codes we identified the following adverse events: any infections or hematomas treated operatively, more finger stiffness than expected, (transient) symptoms of nerve dysfunction after surgery (recorded in the medical record), delayed carpal tunnel release, malunion (as defined and coded by the treating surgeon), reoperation for loss of alignment, hardware removal, and tendon ruptures. We calculated an overall adverse event rate and subsequent surgery rate. Time to carpal tunnel release, hardware removal and tendon rupture were also recorded. Investigators not involved with the treatment of the patients extracted all measures, blinded to patient's reduction status. Among the 1511 included patients we recorded 231 adverse events in 211 patients (14\%) and 106 additional surgeries in 88 patients (5.8\%) after index open reduction and internal fixation.

\section{Statistical analysis}

Frequencies were used to describe dichotomous variables; continuous variables are reported as means and standard deviations. Discrete variables were compared by Fisher exact test, continuous and discrete variables by Student t-test. Matched radiographs were compared by McNemar test and paired t-test.

To account for potential differences in baseline characteristics, we created two multilevel multivariable logistic regression models to measure the association between reduction status and (1) overall complications and (2) subsequent procedures. To account for correlation of patients treated per surgeon we included a random intercept term that varies at the level of each surgeon, we based our estimates on 10 integration points.

The 52 surgeons that treated less than 40 patients were grouped, so that all surgeons (clusters) had sufficient patients $(n>40)$ according to previously recommended sample sizes for this type of model to reduce bias. ${ }^{4}$ We adjusted models using all available patient and treatment related variables. Odds ratios (95\% confidence intervals) and $P$ values are reported and used together as a measure of overall significance.

One study previously reported an adverse event rate of $11.3 \%$ after volar plate fixation of distal radius fractures. ${ }^{5}$ Assuming a relevant difference of $20 \%$ in adverse events between reduced and unreduced fractures (i.e. a rate of $31.3 \%$ in our unreduced patients), an a priori sample size calculation indicated that a sample size of 150 with an allocation ratio of 0.5 (reduced $n=100$, unreduced $n=50$ ) would provide $80 \%$ power to show a difference in adverse event rates with alpha set at 0.05 (Z-test, two independent proportions, G*Power 3.1).

\section{RESULTS}

Accounting for potential differences in baseline characteristic by multivariable analysis, we found no difference in specific or overall rates of adverse events (adjusted odds ratio unreduced fractures 1.1, 95\% confidence interval 0.56-2.3, $P=0.74$ ) and subsequent surgeries (adjusted odds ratio unreduced fractures $0.71,95 \%$ confidence interval $0.25-2.0, P=0.38$ ) between reduced and unreduced fractures. We found no difference in time to carpal tunnel release, plate removal, or tendon rupture (Table $2 \& 3$ ).

Eleven patients had a total of 11 infections (10 reduced, 1 unreduced fracture), 2 with associated hematomas (both reduced fractures). 
Table 2. Complications of operated reduced and unreduced distal radius fractures

\begin{tabular}{|c|c|c|c|c|}
\hline Complication & Total & Reduced & Unreduced & $P$ value \\
\hline Infection & $0.73 \%(11)$ & $0.71 \%(10)$ & $0.98 \%(1)$ & 0.54 \\
\hline Hematoma & $0.13 \%(2)$ & $0.14 \%(2)$ & 0 & 1.0 \\
\hline - Incision and drainage* & $0.40 \%(6)$ & $0.43 \%(6)$ & 0 & 1.0 \\
\hline Stiffness & $1.9 \%(29)$ & $1.8 \%(26)$ & $2.9 \%(3)$ & 0.44 \\
\hline Neuropathology & $11 \%(171)$ & $11 \%(159)$ & $12 \%(12)$ & 0.87 \\
\hline - For which CTR & $1.5 \%(22)$ & $1.5 \%(21)$ & $0.98 \%(1)$ & 1.0 \\
\hline - Time to CTR & $106( \pm 111)$ & $105( \pm 114)$ & 125 & 0.87 \\
\hline Malunion & $0.53 \%(8)$ & $0.57 \%(8)$ & 0 & 1.0 \\
\hline Loss of alignment & $0.46 \%(7)$ & $0.50 \%(7)$ & 0 & 1.0 \\
\hline Plate removal & $4.2 \%(63)$ & $4.2 \%(59)$ & $3.9 \%(4)$ & 1.0 \\
\hline - Time to removal & $178( \pm 103)$ & $176( \pm 104)$ & $207( \pm 85)$ & 0.57 \\
\hline Tendon rupture & $0.20 \%(3)$ & $0.14 \%(2)$ & $0.98 \%(1)$ & 0.19 \\
\hline - Time to rupture & $171( \pm 145)$ & $194( \pm 197)$ & 125 & 0.82 \\
\hline One or more adverse events & $14 \%(211)$ & $14 \%(195)$ & $16 \%(16)$ & 0.56 \\
\hline - Unadjusted odds ratio (95\% Cl) & & reference value & $1.2(0.67-2.0)$ & 0.61 \\
\hline Subsequent surgeries & $5.8 \%(88)$ & $6.0 \%(84)$ & $3.9 \%(4)$ & 0.51 \\
\hline - Unadjusted odds ratio (95\% Cl) & & reference value & $0.64(0.23-1.8)$ & 0.37 \\
\hline
\end{tabular}

CTR = carpal tunnel release; time to in days ( \pm standard deviation); $\mathrm{Cl}=$ confidence interval. $\mathrm{P}$ value $<0.05$ indicates statistically significant difference.

*Incision and drainage: related to infection and/or hematoma. 
Table 3. Mixed effects logistic regression for overall complications and subsequent surgeries

\begin{tabular}{|c|c|c|c|}
\hline Overall complications & $\begin{array}{l}\text { Odds Ratio } \\
\text { (95\% Confidence Interval) }\end{array}$ & Standard error & Pvalue \\
\hline Unreduced fractures & $1.1(0.56-2.3)$ & 0.40 & 0.74 \\
\hline Age & $1.015(1.002-1.027)$ & 0.0062 & 0.015 \\
\hline Male sex & $1.0(0.68-1.6)$ & 0.22 & 0.90 \\
\hline \multicolumn{4}{|l|}{ Fracture type } \\
\hline - Extra-articular fracture & reference value & & \\
\hline - Intra-articular, 2 fragments & $0.77(0.45-1.29)$ & 0.20 & 0.32 \\
\hline - Intra-articular, $\geq 3$ fragments & $1.1(0.70-1.7)$ & 0.24 & 0.74 \\
\hline First presentation at outside Emergency Department & $1.0(0.95-1.0)$ & 0.19 & 0.37 \\
\hline Days between first presentation and surgery & $0.98(0.95-1.0)$ & 0.019 & 0.37 \\
\hline Treatment with volar locking plate & $1.1(0.44-3.0)$ & 0.56 & 0.78 \\
\hline Concomittant carpal tunnel release & $30(19-45)$ & 6.3 & $<0.001$ \\
\hline \multicolumn{4}{|l|}{ Subsequent surgeries } \\
\hline Unreduced fractures & $0.71(0.25-2.0)$ & 0.38 & 0.53 \\
\hline Age & $1.0(0.99-1.0)$ & 0.0073 & 0.95 \\
\hline Male sex & $0.98(0.59-1.6)$ & 0.25 & 0.95 \\
\hline \multicolumn{4}{|l|}{ Fracture type } \\
\hline - Extra-articular fracture & reference value & & \\
\hline - Intra-articular, 2 fragments & $0.64(0.33-1.2)$ & 0.22 & 0.19 \\
\hline - Intra-articular, $\geq 3$ fragments & $1.1(0.65-1.9)$ & 0.29 & 0.72 \\
\hline First presentation at outside Emergency Department & $0.73(0.46-1.2)$ & 0.17 & 0.19 \\
\hline Days between first presentation and surgery & $0.97(0.92-1.0)$ & 0.024 & 0.19 \\
\hline Treatment with volar locking plate & $1.8(0.42-7.5)$ & 1.3 & 0.44 \\
\hline Concomittant carpal tunnel release & $1.7(0.93-3.1)$ & 0.52 & 0.088 \\
\hline
\end{tabular}

\section{DISCUSSION}

Closed reduction and cast or splint immobilization is the standard initial treatment for most fractures of the distal radius ${ }^{6}$. A subset of fractures is considered for operative treatment prior to attempted reduction. The aim of our study was to assess if unreduced fractures can be left unreduced without safety concerns when the plan is for surgery and there is no wound, skin tenting, or neuropathy. We found no differences in overall and specific adverse events and subsequent surgeries between patients treated with manipulative reduction compared to those that were splinted without reduction prior to surgery.

This study has some limitations. First, in this retrospective study we could not measure symptoms and disability, overall satisfaction or pain levels as we might in a prospective study. Secondly, we did not correct 
for possible differences in ulnarward inclination by multivariable analysis, as we regarded the average $4^{\circ}$ more ulnarward inclination in unreduced fractures as clinically irrelevant. Thirdly, a limitation of any large database study is reliance on prior collected data, some of which is likely inaccurate. We reviewed the medical record to verify all adverse events. Fourthly, our data should not be used as a measure of the final results of treatment of distal radius fractures as our 1 year follow-up might be too short for some late complications, such as tendon ruptures, to develop. In addition, we included 64 treating surgeons. This could have resulted in selection bias regarding which patients were reduced, which were operated, and the surgical outcomes. We account for variation by specific surgeon in the statistical analysis. A benefit of the large number of surgeons is that this reflects what is done in actual practice; it increases the likelihood that our data applies to the average surgeon and the average patient. Our study does not address specific criteria used reliably. Rather, we studied what a large group of surgeons does in actual practice.

We don't want this study to encourage surgery prior to an attempt at manipulative reduction and immobilization. Based on estimated probability of loss of alignment with non-operative treatment, the surgeon offers and the patient determines their preferences for operative or non-operative treatment. But when the patient and surgeon prefer surgical treatment no matter what manipulative reduction might achieve, and there are no wounds, skin tenting, or neuropathy, our experience and data suggest that it's reasonable to consider foregoing an attempt at reduction. The Lafontaine criteria ([1] > 20 dorsal angulation; [2] dorsal comminution; [3] intra-articular involvement; [4] associated ulna fracture; [5] age $>60$ years) ${ }^{7}$ and the probability calculator of Mackenney and McQueen' are intended to help determine the probability of loss of alignment, but need additional prospective verification. Once these probabilities are determined, the preferences and circumstances of the patient need to be taken into account as well.

The decision to defer reduction and schedule surgery seemed subjective and imprecise in our patients. Often it was circumstantial. For instance, some patients had splinting without reduction in an outside care setting where the caregivers could not provide reduction. When they were seen in the office, the decision was made to proceed directly to surgery instead of attempting a reduction. Other times, patients seen at night were admitted for surgery in the morning and manipulation was deferred.

We found an overall adverse event rate of 15\% (231 of 1511). Two prior large series, including over 500 patients, reported overall rates of $8 \%(47 \text { of } 594)^{8}$ and $11 \%(75 \text { of } 665)^{5}$ after distal radius fracture plating. Our rate may be higher because we incorporated stiffness, transient nerve dysfunction and any subsequent surgery in our count of adverse events and subsequent surgeries.

Our results suggest no difference in adverse events and subsequent surgeries the first year after surgery between reduced and unreduced fractures of the distal radius that are treated operatively. Conscious of the retrospective nature of our study and the need for additional prospective study, this raises the possibility that patients with fractures choosing operative treatment based on the initial post-injury radiographs can avoid closed reduction when surgery is planned within an average of 5 days (range 0 to 21 days).

\section{ACKNOWLEDGMENTS}

The authors would like to thank Nicky Stoop and Kirsten Verheij for their help with the revisions of the manuscript and Mariano Menendez for his statistical support. 


\section{REFERENCES}

1. Mackenney PJ, McQueen MM, Elton R. Prediction of instability in distal radial fractures. J Bone Joint Surg Am. 2006;88(9):1944-1951.

2. Arora R, Lutz M, Deml C, Krappinger D, Haug L, Gabl M. A prospective randomized trial comparing nonoperative treatment with volar locking plate fixation for displaced and unstable distal radial fractures in patients sixty-five years of age and older. J Bone Joint Surg Am. 2011;93(23):2146-2153.

3. Medoff RJ. Essential radiographic evaluation for distal radius fractures. Hand Clin. 2005;21(3):279-288.

4. Moineddin R, Matheson FI, Glazier RH. A simulation study of sample size for multilevel logistic regression models. BMC Med Res Methodol. 2007;7:34.

5. Esenwein P, Sonderegger J, Gruenert J, Ellenrieder B, Tawfik J, Jakubietz M. Complications following palmar plate fixation of distal radius fractures: a review of 665 cases. Arch Orthop Trauma Surg. 2013;133(8):1155-1162.

6. Chung KC, Shauver MJ, Birkmeyer JD. Trends in the United States in the treatment of distal radial fractures in the elderly. J Bone Joint Surg Am. 2009;91(8):1868-1873.

7. Lafontaine M, Hardy D, Delince P. Stability assessment of distal radius fractures. Injury. 1989;20(4):208-210.

8. Soong M, van Leerdam R, Guitton TG, Got C, Katarincic J, Ring D. Fracture of the distal radius: risk factors for complications after locked volar plate fixation. J Hand Surg Am. 2011;36(1):3-9. 
CHAPTER

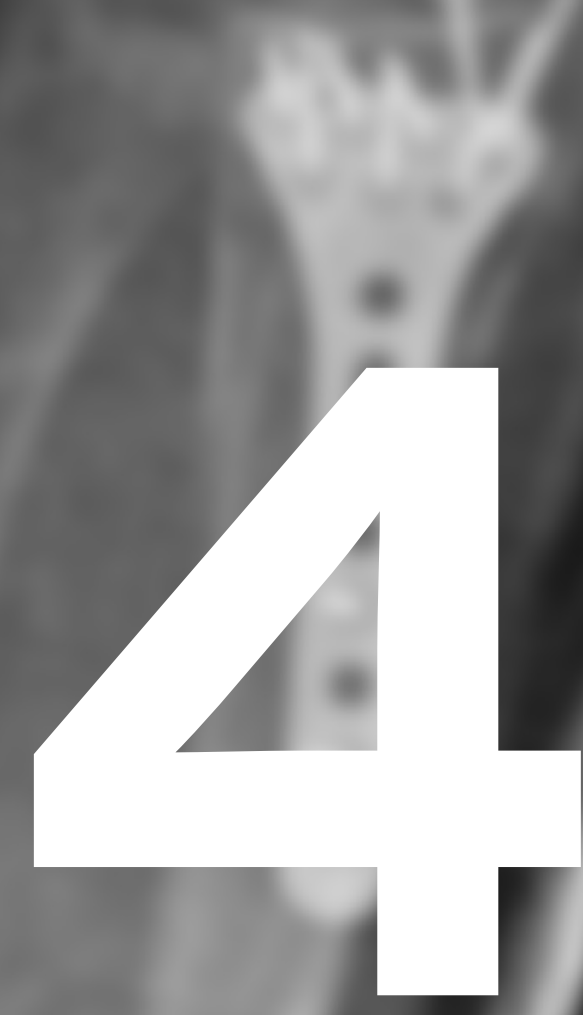




\section{Evaluation of radiographic}

fracture position one year after

variable angle locking volar distal

radius plating. A prospective

multicenter case series.

T. Teunis

A. Joeris

K.D. Schaser

G. Fronhöfer

R. Babst

M. Langer

A. Platz

A. Schierz

J. Jupiter

D. Rikli

Submitted

Presented at the International Wrist Investigator's Workshop (2015).

Work supported by AO TK Trauma Network. 
Importance Treatment with a variable angle locking plate can achieve anatomic reduction in intra-articular distal radius fractures, but it's unknown to what extent reduction is maintained as measured by CT.

Objectives To compare (1) postoperative radiographic fracture position with the position one year after surgery; (2) fracture position and functional outcome in single and double distal screw rows, and (3) to compare gap and step off measured on radiographs and CT scans.

Design Prospective cohort study.

Setting 6 hospitals in Switzerland and Germany.

Participants 73 patients with a distal radius fracture requiring surgery, of whom 66 patients (90\%) were available at one year.

Exposures Variable angle locking volar distal radius plate.

Main Outcomes On posteroanterior radiographs: radial height, ulnarward inclination, ulnar variance, gap, and step off. On lateral radiographs: palmar tilt, gap, step off, scapholunate angle, teardrop angle, and anteroposterior distance. On CT: gap on sagittal, frontal and axial views and step off on sagittal and frontal views.

Results We found a small (less than $2 \mathrm{~mm}$ or 2 degrees) but statistically significant change in several measures. Only one patient had more than $3^{\circ}$ loss of palmar tilt, and only one patient experienced an increase in gap more than $3 \mathrm{~mm}$. Only one patient worsened more than $3 \mathrm{~mm}$ on each CT measure. Accounting for inter-observer agreement, this is probably within measurement error. We found no difference in change in fracture position or range of motion, grip strength or patient reported outcome between one or two distal screw rows. Gap and step-off measures on radiographs and CT scans only show limited correlation.

Conclusion Only minimal change in reduction can be expected after volar plate fixation. We recommend using only one screw row routinely, limiting costs, surgery time and misplacement of screws.

Level of Evidence Therapeutic level IV 


\section{INTRODUCTION}

In intra-articular distal radius fractures anatomic reduction can be achieved via open reduction and internal fixation with a volar locking plate.' More recently, variable angle locking plates (VA LCP) have been developed to potentially better address individual fracture fragments. Adverse events after volar locking plating include intra-articular screw placement, tendinitis, tendon rupture, and loss of reduction ${ }^{2-4}$ and affect between $2.5 \%$ and $32 \%$ of all patients. ${ }^{5,6}$

The goal of open reduction and internal fixation is to improve alignment, but it's unknown to what extent reduction is maintained measured by computed tomography (CT) after treatment with a VA LCP. This prospective case-series evaluates the performance of a VA LCP in the treatment of intra-articular fractures of the distal radius. The primary aim of this study is to compare postoperative radiographic fracture position with the position one year after surgery. To fully address any change, we also determined inter- and intra-observer agreement of the radiographic measures. Secondary aims of the study are (1) to compare fracture position and functional outcome in single and double distal screw rows, and (2) to compare gap and step off measured on radiographs and $\mathrm{CT}$ scans.

\section{PATIENTS \& METHODS}

\section{Study design}

Six centers participated in our prospective study and institutional review board approval was obtained at each center. We included adult patients ( $\geq 18$ years) with a closed, partial or complete articular distal radius fracture ( $\mathrm{AO}$ fracture type $\mathrm{B} 3, \mathrm{Cl}$ to $\mathrm{C} 3$, confirmed by $\mathrm{CT}$ ) treated with a $2.4 \mathrm{~mm}$ Variable Angle Locking Two-Column Volar Distal Radius Plate ${ }^{\circ}$ (VA LCP, Synthes, Oberdorf, Switzerland). ${ }^{7}$ Exclusion criteria were: (1) previous ipsilateral distal radius fracture; (2) other ipsilateral fractures except the ulna or polytrauma; (3) pathologic fracture or active malignancy; (4) inability to complete enrollment forms due to any mental status or language problems; and (5) (potentially) pregnant or breastfeeding women. The surgical technique for the application of the VA LCP plate has been described previously. ${ }^{8}$ Postoperative treatment was left at discretion of the treating surgeon. In general this involved reducing the bandages on the first postoperative visit and starting early active movement exercises, if necessary under the supervision of a hand therapist.

\section{Radiographic measurements}

We obtained fracture radiographs and CT scans pre- and postoperatively (within 10 working days), and 12 months (range 11 to 13 months) after surgery. Standardized methods for radiograph and CT acquisition and analysis are outlined in our research protocol. The following parameters were recorded on posteroanterior radiographs (Appendix 1): radial height, ulnarward inclination (also referred to as radial inclination), ulnar variance, gap, and step off. On lateral radiographs (Appendix 2) we measured: palmar tilt, gap, step off, scapholunate angle, teardrop angle, and anteroposterior distance..$^{910}$ On CT we measured gap on sagittal, frontal and axial views and step off on sagittal and frontal views (Figures 1, 2 and 3). ${ }^{11,12}$ An independent radiologist assessed all radiographs; the primary author assessed all CT scans. 

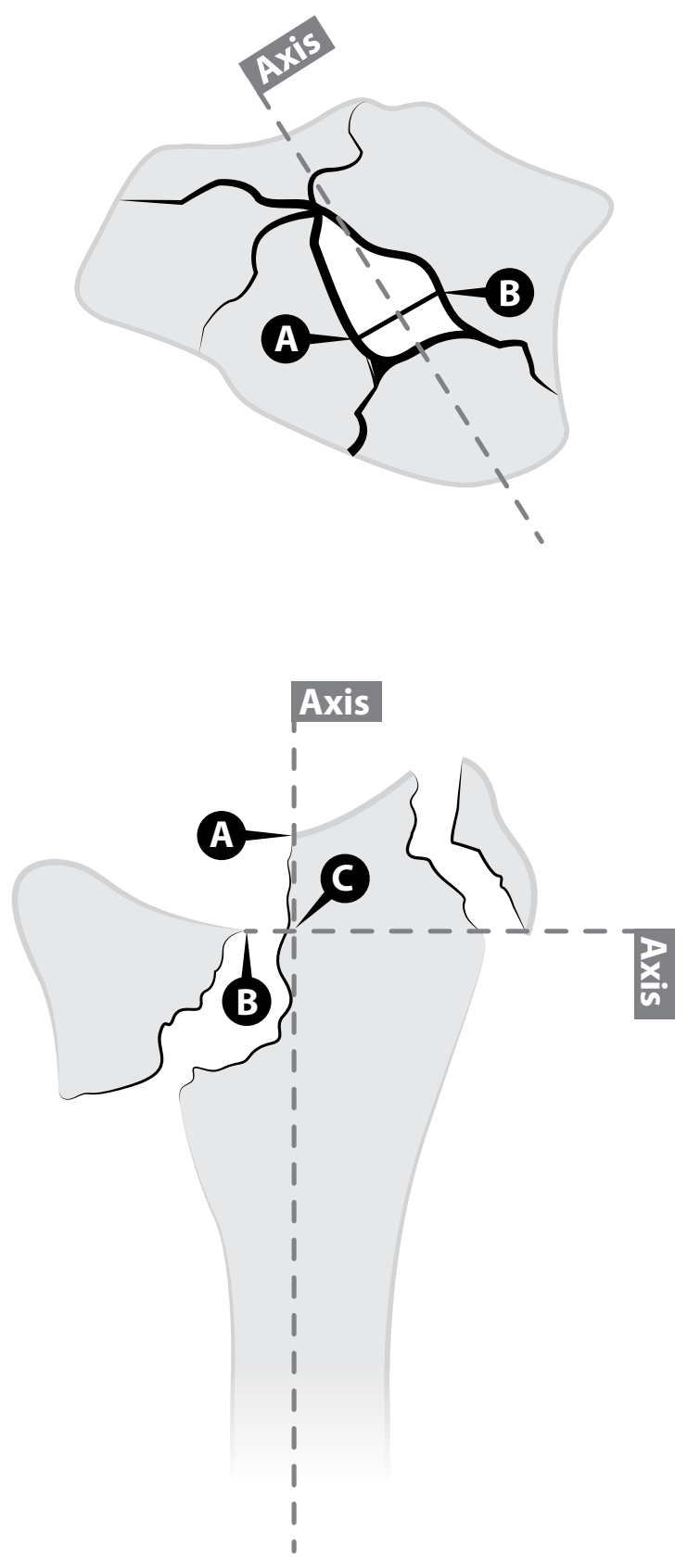

FIGURE 1: MEASUREMENTS ON AXIAL

\section{CT WITH CENTRAL DEPRESSION.}

Gap on axial CT scan is measured by

the largest distance between fragments,

perpendicular to the axis of the gap.

FIGURE 2: MEASUREMENTS ON FRONTAL CT (LONGITUDINAL AXIS METHOD).

Step off (A to $C$ ) is measured by drawing a line along the axis of the distal radius that intersects one fragment's corner (A). The height of the adjacent depressed articular surface on this line is marked (C). Gap (C to B) is measured on a line, perpendicular to the axis, to the next articular fragment (B. One selects the CT slice with the largest gap and step off. 


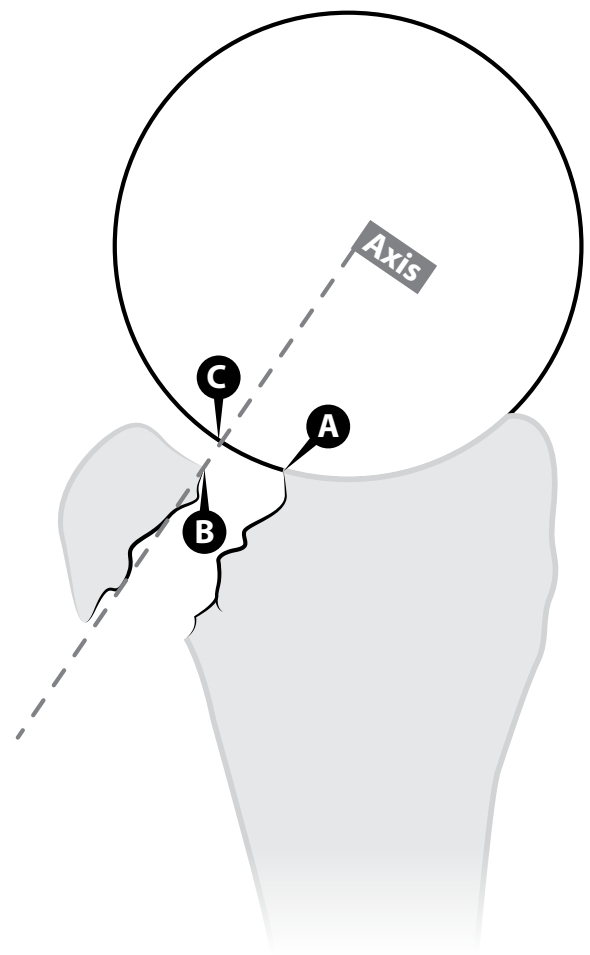

\section{FIGURE 3: GAP AND STEP OFF MEASURED ON SAGITTAL CT (ARC METHOD).}

A circle is drawn along the curvature of the greatest remaining articular surface of the distal radius. A line is drawn (marked axis) through the geometric center of the circle, passing through to the next articular fragment. Step off is measured between where the axis intersects the circle $(C)$ and the bone $(A)$. Gap is measured between the corner of the greatest remaining articular fragment (B) and (C). One selects the CT slice with the largest gap and step off.

\section{Functional measurements}

A site-specific research coordinator, not involved with patient care, obtained informed consent, administered the questionnaires, and performed the motion and grip measurements. We calculated a composite value for wrist range of motion by summing the ranges of flexion/extension, radial/ulnar deviation and pronation/ supination.

We assessed disability by the patient self-assessment of wrist function questionnaire (PRWE). PRWE SCore ranges from 0 to 100, where higher scores indicated greater pain and disability. ${ }^{13}$ To assess health-related quality of life we used the EuroQoL5 (EQ5D). The EQ5D utility score was computed through a weighted regression-based algorithm, with a higher score indicating greater quality of life (eq5d command, Stata 13.0, StataCorp LP, Texas, USA). ${ }^{14,15}$

At enrollment we recorded sociodemographics. Upper limb function, quality of life, wrist range of motion, and grip strength were assessed at 12 weeks (range 8 to 15 weeks) and 12 months (range 11 to 13 months) after fracture.

\section{Patient Demographics}

Between September 8, 2010 and January 22, 2013 we approached 81 consecutive patients to participate in our study. Eight patients were not eligible after surgery because they were treated with additional plates ( $n=3$ ) or a dorsal plate only $(n=4)$ and because once accidently a different volar plate was used. Of the 73 remaining patients, 66 (90\%) were available at one year. Four patients chose to withdraw from the study and three patients were excluded due to non-compliance. Average age at enrollment was 61 ( \pm standard deviation [SD] 
15) years range 25 to 88 years; 24 (33\%) patients were male. The majority (66\%, 48 patients) fractured their radius due to a fall from standing height, and 44\% (32 fractures) were AO type C3 (Table 1).

\section{Table 1. Baseline characteristics}

\section{Variable}

Enrolled

Available for follow-up:

- Post operative

- 12 weeks

$\cdot 1$ year

Age (range)

Male

Body mass index

Tobacco use

Comorbidity

Mechanism of injury

- Fall from standing height

- Traffic accident

- 0ther

Injured dominant side

AO fracture classication

- B3

- C1

- $C 2$

- $C 3$

Ulna fracture

- Of which treated surgically

Central articular depression

Initial treatment with external fixator

Closed reduction priot to surgery

Days from injury to hospital admission (range)

Days form injury to surgery (range)

\section{Measurement}

73

$100 \%(73)$

$96 \%(70)$

$90 \%(66)$

$61 \pm 15(25-88)$

$33 \%(24)$

$25 \pm 4.9$

$11 \%(8)$

$67 \%(49)$

$66 \%(48)$

$18 \%(13)$

$16 \%(12)$

$39 \%(24)^{*}$

$8 \%(6)$

$21 \%(15)$

$27 \%(20)$

$44 \%$ (32)

$44 \%$ (32)

$9 \%(3)$

$13 \%(9)$

$27 \%(20)$

$42 \%$ (31)

$2 \pm 4(0-11)$

$4 \pm 3(0-11)$

Continous data as mean ( \pm standard deviation), discrete data as percentage (number); ${ }^{*} 11$ missings. 


\section{Statistical analysis}

A priori sample size calculation with alpha set at 0.05 indicated that if we found no change in alignment in 59 patients, the rate within the general population would be below $5 \%$.

Continuous data is presented as mean $( \pm \mathrm{SD})$, discrete data as percentage and number. Paired Student $\mathrm{t}$-tests were used to compare fracture position after surgery and at 1 year and to compare change in fracture position and outcomes between single and double distal screw rows.

Difference in fracture position might be attributable to measurement error. To assess the accuracy of all radiographic measures, two observers measured 45 radiographs and CT scans twice. We calculated inter- and intra-observer variability by intra-class correlation coefficients for a two-way random effects model. This model assumes all raters rate the same set of fractures and that they are sampled randomly from a larger population. We report the absolute agreement, i.e. how much each measurement performed per observer differed from the other observer. Sample size calculation indicated 42 samples would provide $80 \%$ power with alpha set at 0.05 , assuming an intra-class correlation coefficient of 0.80 , with a confidence interval width of 0.22 .

We calculated Pearson correlations to assess similarity of gap and step off measurements on radiographs and CT scans. Because correlations do not account for systematic variance, we also created Bland-Altman plots ${ }^{16}$. A little random noise was added to prevent overlying data points.

We considered $p<0.05$ significant.

\section{Protocol deviation}

Instead of an accuracy of $0.5 \mathrm{~mm}$ we determined radiographic measures with $1 \mathrm{~mm}$ accuracy. The study protocol is registered at ClinicalTrials.gov, protocol number NCT01 103297.

\section{Source of Funding}

The presented clinical investigation was performed with the support of the AO TK Trauma Network.

\section{RESULTS}

Radiological measures

We found statistically significant change comparing several postoperative radiographic and CT measures with the position one year after surgery. However, mean change was less than $1 \mathrm{~mm}$ or degree, or less than 2 degrees for scapholunate and teardrop angle. Only one patient had more than $3^{\circ}$ loss of palmar tilt, and only one patient experienced an increase in gap more than $3 \mathrm{~mm}$. Only one patient worsened more than $3 \mathrm{~mm}$ on each CT measure, except for frontal step off where no patient worsened more than $3 \mathrm{~mm}$. Inter-observer agreement varied. Gap and step-off measures overall were least reliable. Ulnar variance was most reliable (Table 2). 
Table 2. Change in fracture position and measurement reliability

\begin{tabular}{|c|c|c|c|c|c|c|}
\hline Radiographic measures & Post-operative & 1 year & Pvalue & Change & $\begin{array}{l}\text { Inter-observer } \\
\text { agreement }\end{array}$ & $\begin{array}{l}\text { Intra-observer } \\
\text { agreement }\end{array}$ \\
\hline \multicolumn{7}{|l|}{ Posteroanterior } \\
\hline Radial height (mm) & $10 \pm 1.0$ & $10 \pm 1.4$ & 0.18 & $0.26 \pm 1.5$ & 0.88 (0.79 to 0.93$)$ & 0.89 (0.80 to 0.94$)$ \\
\hline Ulnarward inclincation $\left({ }^{\circ}\right)$ & $20 \pm 0.93$ & $20 \pm 2.0$ & 0.51 & $0.17 \pm 2.1$ & 0.87 (0.63 to 0.95$)$ & 0.91 (0.84 to 0.95$)$ \\
\hline Ulnar variance (mm) & $-2.0 \pm 0.67$ & $-1.7 \pm 0.59$ & 0.0015 & $0.28 \pm 0.57$ & $0.94(0.90$ to 0.97$)$ & 0.91 (0.84 to 0.95$)$ \\
\hline Gap (mm) & $1.4 \pm 0.56$ & $1.1 \pm 0.64$ & $<0.001$ & $-0.37 \pm 0.65$ & 0.77 (0.63 to 0.87$)$ & $0.44(0.17$ to 0.65$)$ \\
\hline Step off (mm) & $1.3 \pm 0.48$ & $0.80 \pm 0.60$ & $<0.001$ & $-0.54 \pm 0.61$ & $0.13(-0.17$ to 0.40$)$ & 0.41 (0.15 to 0.62$)$ \\
\hline \multicolumn{7}{|l|}{ Lateral } \\
\hline Palmar tilt $\left(^{\circ}\right)$ & $9.1 \pm 0.79$ & $9.6 \pm 0.63$ & $<0.001$ & $0.51 \pm 1.0$ & $0.82(0.70$ to 0.90$)$ & 0.67 (0.48 to 0.81$)$ \\
\hline Gap (mm) & $1.6 \pm 0.53$ & $1.2 \pm 1.3$ & 0.041 & $-0.32 \pm 1.3$ & $0.17(-0.11$ to 0.43$)$ & $0.22(-0.073$ to 0.49$)$ \\
\hline Step off (mm) & $1.6 \pm 0.68$ & $0.91 \pm 0.65$ & $<0.001$ & $-0.68 \pm 0.81$ & $-0.036(-0.32$ to 0.26$)$ & $0.74(0.57$ to 0.85$)$ \\
\hline Scapholunate angle $\left({ }^{\circ}\right)$ & $52 \pm 6.3$ & $53 \pm 5.1$ & 0.066 & $1.3 \pm 5.8$ & $0.58(0.35$ to 0.74$)$ & $0.71(0.53$ to 0.83$)$ \\
\hline Teardrop angle $\left({ }^{\circ}\right)$ & $65 \pm 3.6$ & $67 \pm 3.2$ & $<0.001$ & $1.8 \pm 2.6$ & $0.66(0.40$ to 0.81$)$ & $0.63(0.42$ to 0.78$)$ \\
\hline Anteroposterior distance (mm) & $17 \pm 0.70$ & $17 \pm 0.64$ & 1.0 & $0 \pm 0.97$ & 0.89 (0.82 to 0.94$)$ & $0.87(0.78$ to 0.93$)$ \\
\hline \multicolumn{7}{|l|}{ CT measures } \\
\hline \multicolumn{7}{|l|}{ Sagittal } \\
\hline Step off (mm) & $1.2 \pm 1.1$ & $1.1 \pm 1.1$ & 0.31 & $-0.14 \pm 1.1$ & $0.56(0.33$ to 0.73$)$ & 0.71 (0.53 to 0.83) \\
\hline Gap (mm) & $3.5 \pm 2.4$ & $3.2 \pm 2.8$ & 0.058 & $-0.41 \pm 1.6$ & 0.85 (0.74 to 0.92$)$ & 0.84 (0.73 to 0.91$)$ \\
\hline \multicolumn{7}{|l|}{ Axial } \\
\hline Gap (mm) & $2.3 \pm 1.3$ & $1.9 \pm 1.7$ & 0.073 & $-0.35 \pm 1.6$ & 0.41 (0.14 to 0.62$)$ & 0.64 (0.43 to 0.79$)$ \\
\hline \multicolumn{7}{|l|}{ Frontal } \\
\hline Step off (mm) & $1.2 \pm 0.96$ & $1.1 \pm 0.99$ & 0.10 & $-0.16 \pm 0.76$ & $0.17(-0.11$ to 0.43$)$ & $0.39(0.12$ to 0.61$)$ \\
\hline Gap (mm) & $4.6 \pm 3.3$ & $3.9 \pm 2.5$ & 0.023 & $-0.61 \pm 2.0$ & $0.45(0.17$ to 0.66$)$ & $0.58(0.22$ to 0.77$)$ \\
\hline
\end{tabular}

\section{Single versus double distal screw rows}

We found no difference in change in fracture position between one and two distal screw rows. There was also no difference in range of motion, grip strength or patient reported outcome at one year (Table 3). 
Table 3. Single versus double tiers

Change in radiographic measures

Posteroanterior

- Radial height $(\mathrm{mm})$
- Ulnarward inclincation $\left(^{\circ}\right)$
- Ulnar variance $(\mathrm{mm})$

- Gap (mm)

- Step off (mm)

Lateral

- Palmar tilt $\left({ }^{\circ}\right)$

- Gap (mm)

- Step off (mm)

- Scapholunate angle $\left(^{\circ}\right)$

- Teardrop angle $\left({ }^{\circ}\right)$

- Anteroposterior distance (mm)

\section{Change in CT measures}

Sagittal

$$
\begin{aligned}
& \text {-Step off (mm) } \\
& \text {-Gap (mm) }
\end{aligned}
$$

$-0.11 \pm 0.78$

$-0.18 \pm 1.4$

$-0.29 \pm 0.79$

Axial

$$
\text { - Gap (mm) }
$$

Two screw rows

P value

$0.037 \pm 0.71$

$0.52 \pm 2.1$

$0.19 \pm 0.56$

$-0.33 \pm 0.62$

$-0.59 \pm 0.64$

$0.67 \pm 0.83$

$-0.44 \pm 0.70$

$-0.78 \pm 0.93$

$2.6 \pm 4.6$

$2.2 \pm 1.9$

$0.26 \pm 0.59$
$0.42 \pm 1.9$

$-0.079 \pm 2.1$

$0.34 \pm 0.75$

$-0.39 \pm 0.68$

$-0.50 \pm 0.60$

$0.39 \pm 1.2$

$-0.24 \pm 1.5$

$-0.61 \pm 0.72$

$0.42 \pm 1.0$

$1.5 \pm 3.0$

$-0.18 \pm 1.1$
0.33

0.26

0.36

0.71

0.55

0.30

0.51

0.40

0.13

0.27

0.068
Frontal

$$
\begin{aligned}
& \text { - Step off (mm) } \\
& \text {-Gap (mm) }
\end{aligned}
$$

$-0.29 \pm 0.64$

$-0.28 \pm 1.6$

$-0.074 \pm 0.84$

$-0.85 \pm 2.2$

\section{Function at 1 year}

Composite range of motion

Grip strength

$317 \pm 40$

$311 \pm 55$

0.62

PRWE

$23 \pm 16$

$5.4 \pm 7.5$

$0.92 \pm 0.11$
$27 \pm 11$

0.25

$9.3 \pm 15$

0.21

$0.92 \pm 0.11$
0.85

0.78

EuroQ0L5

Data as mean ( \pm standard deviation); PRWE = Patient self-assessment of wrist function questionnaire. 


\section{Similarity step off and gap measures}

Step off on posteroanterior radiographs showed a small correlation with step off measured on frontal CT $(r=0.16, p=0.025)$. Later step off and sagittal CT step-off correlated better ( $r=0.25, p=0.005)$. Cap measured on posteroanterior radiographs correlated with frontal CT gap $(r=0.33, p<0.001)$. Lateral gap correlated with sagittal CT gap ( $r=0.32, p<0.001)$. Bland-Altman plots showed systemic differences. The funnel shape in all plots indicates that when mean step off or gap increases, the difference between computed tomography and radiographic measurements increases as well (Appendix $3 A$ to $D$ ). The decreasing trend seen in plots for gap deformity indicates that when the mean gap increases, computed tomography consistently shows a larger gap than radiographs.

\section{DISCUSSION}

Intra-articular distal radius fractures are commonly treated with volar locking plates.' Treatment aims to improve fracture alignment. However, the extent to which reduction is maintained after variable angle locking plating measured by computed tomography is unknown. We found statistical significant change in several measures, but these changes probably are well within measurement error. Our results indicate that only minimal change in fracture position is to be expected after fixation with a volar locking plate.

This study has several limitations. First, several surgeons at different locations performed the surgeries and the patients were evaluated by a variety of individuals. We carefully evaluated all data points, and those outside the $95 \%$ data distribution were checked again to confirm accuracy. Secondly, we categorized change in fracture position in 1 millimeter and degrees. Small changes might be attributable to measurement error or positioning during radiographic evaluation. However, standardized methods for radiograph acquisition are outlined in our research protocol.

Comparing postoperative radiographic and CT measures with the position one year after surgery, we found a statistically significant change in several measures. However, mean change was less than $2 \mathrm{~mm}$ or degrees with small standard deviations. Accounting for inter-observer agreement, this is probably within measurement error. Only a few fractures had obvious change. Even for the most reliable measure of ulnar variance (intra-class correlation 0.94$)$ mean change was significant $(p<0.0015)$ but $0.28 \mathrm{~mm}( \pm 0.57 \mathrm{~mm})$. Three other case-series looking at volar locking plates also found radial height, ulnarward inclincation, and palmar tilt to be stable after surgery. ${ }^{8,17,18}$ However one of the studies still found that 13 out of 40 patients (33\%) had postoperative fracture collapse to some degree, resulting in screw penetration in the radiocarpal joint in 11 patients. The higher rate of collapse might be attributable to a higher proportion of women $(87 \%$ [ $n=35]$ vs. $67 \%$ [ $n=49]$ in our series) and the associated osteopenia with female sex. ${ }^{18}$ To our knowledge no other study assessed change in alignment on CT after volar plate fixation of the distal radius fracture. Our results indicate that after fixation with a variable angle locking plate fractures may settle somewhat, but only minimal change can be expected. We found no difference in change in fracture position - if there was any - between one and two distal screw rows; also there was no difference in range of motion, grip strength or patient reported outcome. Conversely, another retrospective study, including 49 patients, found that volar tilt and ulnar variance were better maintained with two screw rows. Similarly to our study they found no difference in function (range of motion and Cooney score). ${ }^{19}$ Another study including 34 matched pairs also found no difference in fracture position. ${ }^{20}$ There does not seem to be a clear benefit to using one or two screw rows regarding impairment or patient reported outcome. However, using only one row of distal screws might lower the cost of surgery, reduce operation time, and limit opportunities for a misplaced or overly long screw. 
Radiographic and computed tomography step off and gap measurements were poorly correlated. We found more patients with $3 \mathrm{~mm}$ displacement on CT than on radiographs. Two previous studies comparing radiographs and CT found CT measurement to be consistently larger than radiographic measures. ${ }^{11,12}$ One explanation is that CT more reliably measures gap deformity. This seems to be true for gap measured on lateral radiographs but not on posteroanterior views (Table 2). Another potential explanation is that on CT one might be measuring gap within a fracture line, which results in overestimation of the actual gap. Studying specific fracture lines measured on both radiographs and computed tomography could solve this issue.

Only limited radiographic change can be expected after variable angle volar locking plate fixation of intraarticular fractures. Future research is recommended to compare different locking techniques in patients with intra-articular distal radius fractures in regards to loss of reduction. Also, it's unclear to what extent loss of reduction affects function and quality of life. We recommend routinely using only one distal screw row, limiting costs, surgery time and misplacement of screws. 


\section{REFERENCES}

1. Wilcke MK, Hammarberg H, Adolphson PY. Epidemiology and changed surgical treatment methods for fractures of the distal radius: a registry analysis of 42,583 patients in Stockholm County, Sweden, 2004-2010. Acta Orthop. 2013;84(3):292-296.

2. Soong M, van Leerdam R, Guitton TG, Got C, Katarincic J, Ring D. Fracture of the distal radius: risk factors for complications after locked volar plate fixation. J Hand Surg Am. 201 1;36(1):3-9.

3. Esenwein P, Sonderegger J, Gruenert J, Ellenrieder B, Tawfik J, Jakubietz M. Complications following palmar plate fixation of distal radius fractures: a review of 665 cases. Arch Orthop Trauma Surg. 2013;133(8):1155-1162.

4. Teunis T, Mulder F, Nota SP, Milne LW, Dyer GS, Ring D. No Difference in Adverse Events Between Surgically Treated Reduced and Unreduced Distal Radius Fractures. J Orthop Trauma. 2015;29(11):521-525.

5. Drobetz H, Kutscha-Lissberg E. Osteosynthesis of distal radial fractures with a volar locking screw plate system. Int Orthop. 2003;27(1):1-6.

6. Kamano M, Koshimune M, Toyama M, Kazuki K. Palmar plating system for Colles' fractures--a preliminary report. J Hand Surg Am. 2005;30(4):750-755.

7. Synthes. Variable Angle LCP Two-Column Volar Distal Radius Plate 2.4. For fragment-specific fracture fixation with variable angle locking technology. 2015; http://synthes.vo.IInwd.net/o16/LLNWMB8/INT Mobile/Synthes International/Product Support Material/legacy_Synthes_PDF/DSEM-TRM-0815-0464_LR.pdf. Accessed October $17,2015$.

8. Jupiter JB, Marent-Huber M, Group LCPS. Operative management of distal radial fractures with 2.4-millimeter locking plates. A multicenter prospective case series. J Bone Joint Surg Am. 2009;91(1):55-65.

9. Kreder HJ, Hanel DP, McKee M, Jupiter J, McGillivary G, Swiontkowski MF. X-ray film measurements for healed distal radius fractures. J Hand Surg Am. 1996;21(1):31-39.

10. Medoff RJ. Essential radiographic evaluation for distal radius fractures. Hand Clin. 2005;21(3):279-288.

11. Cole RJ, Bindra RR, Evanoff BA, Gilula LA, Yamaguchi K, Gelberman RH. Radiographic evaluation of osseous displacement following intra-articular fractures of the distal radius: reliability of plain radiography versus computed tomography. J Hand Surg Am. 1997;22(5):792-800.

12. Arora S, Grover SB, Batra S, Sharma VK. Comparative evaluation of postreduction intra-articular distal radial fractures by radiographs and multidetector computed tomography. J Bone Joint Surg Am. 2010;92(15):2523-2532.

13. MacDermid JC, Turgeon T, Richards RS, Beadle M, Roth JH. Patient rating of wrist pain and disability: a reliable and valid measurement tool. J Orthop Trauma. 1998;12(8):577-586.

14. Ramos-Goñi JM, Rivero-Arias O. eq5d: A command to calculate index values for the EQ-5D quality-of-life instrument. Stata Journal. 2011;11(1):120-125.

15. EuroQol G. EuroQol--a new facility for the measurement of health-related quality of life. Health Policy. 1990;16(3):199208.

16. Bland JM, Altman DG. Statistical methods for assessing agreement between two methods of clinical measurement. Lancet. 1986;1 (8476):307-310.

17. Figl M, Weninger P, Liska M, Hofbauer M, Leixnering M. Volar fixed-angle plate osteosynthesis of unstable distal radius fractures: 12 months results. Arch Orthop Trauma Surg. 2009;129(5):661-669.

18. Knight D, Hajducka C, Will E, McQueen M. Locked volar plating for unstable distal radial fractures: clinical and radiological outcomes. Injury. 2010;41(2):184-189.

19. Kawasaki K, Nemoto T, Inagaki K, Tomita K, Ueno Y. Variable-angle locking plate with or without double-tiered subchondral support procedure in the treatment of intra-articular distal radius fracture. J Orthop Traumatol. 2014;15(4):271-274

20. Neuhaus V, Badri O, Ferree S, Bot AG, Ring DC, Mudgal CS. Radiographic alignment of unstable distal radius fractures fixed with 1 or 2 rows of screws in volar locking plates. J Hand Surg Am. 2013;38(2):297-301 


\section{APPENDICES}

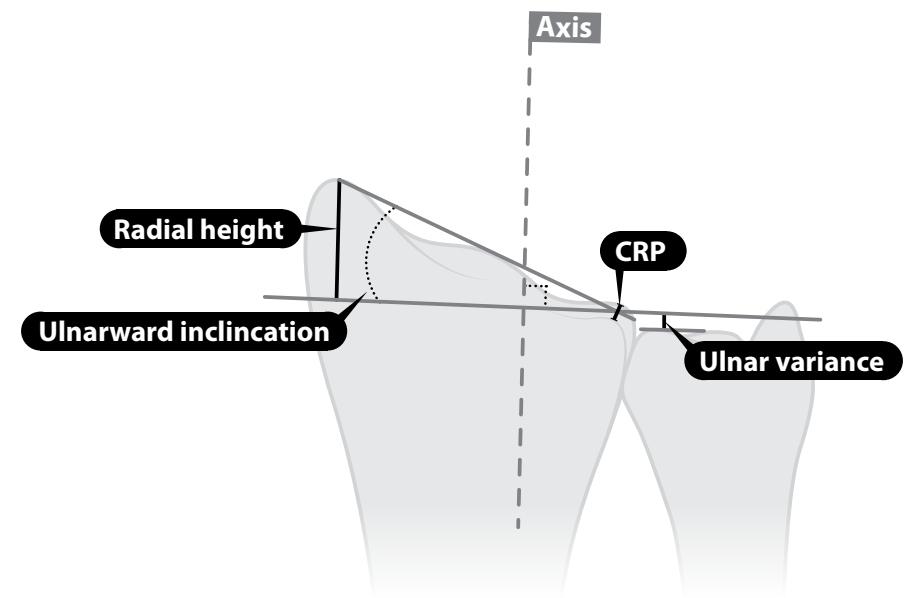

\section{APPENDIX 1: MEASUREMENTS ON POSTEROANTERIOR RADIOGRAPHS.}

The central reference point (CRP) is the point midway between the volar and dorsal ulnar corners, this eliminates variation caused by dorsal and volar angulation. Ulnarward inclination is also referred to as radial inclination. Gap and step off on PA radiographs is measured by the axis method, see figure 2 .

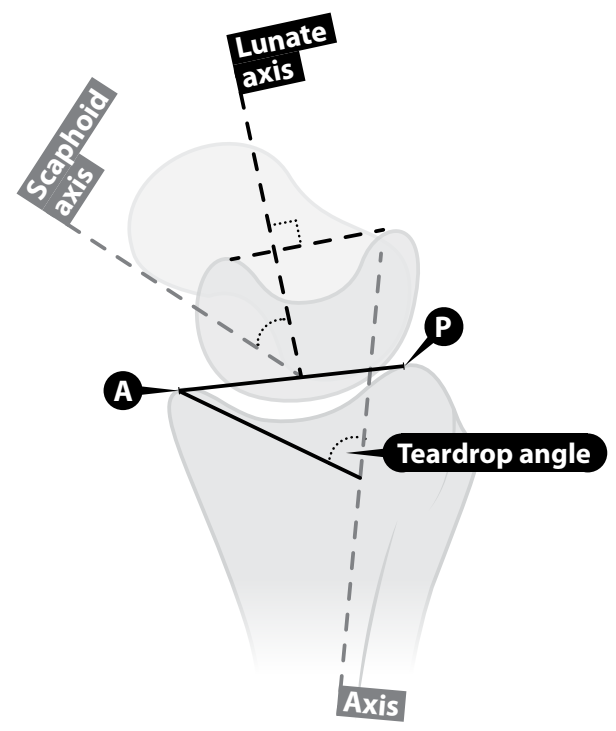

APPENDIX 2: MEASUREMENTS ON LATERAL RADIOGRAPHS.

The scapholunate angle is the angle between the scaphoid and lunate axis. Anteroposterior distance is measured between A and P. Volar tilt is measured as the angle between a line perpendicular to the axis and $\mathrm{A}$ to $\mathrm{P}$. 


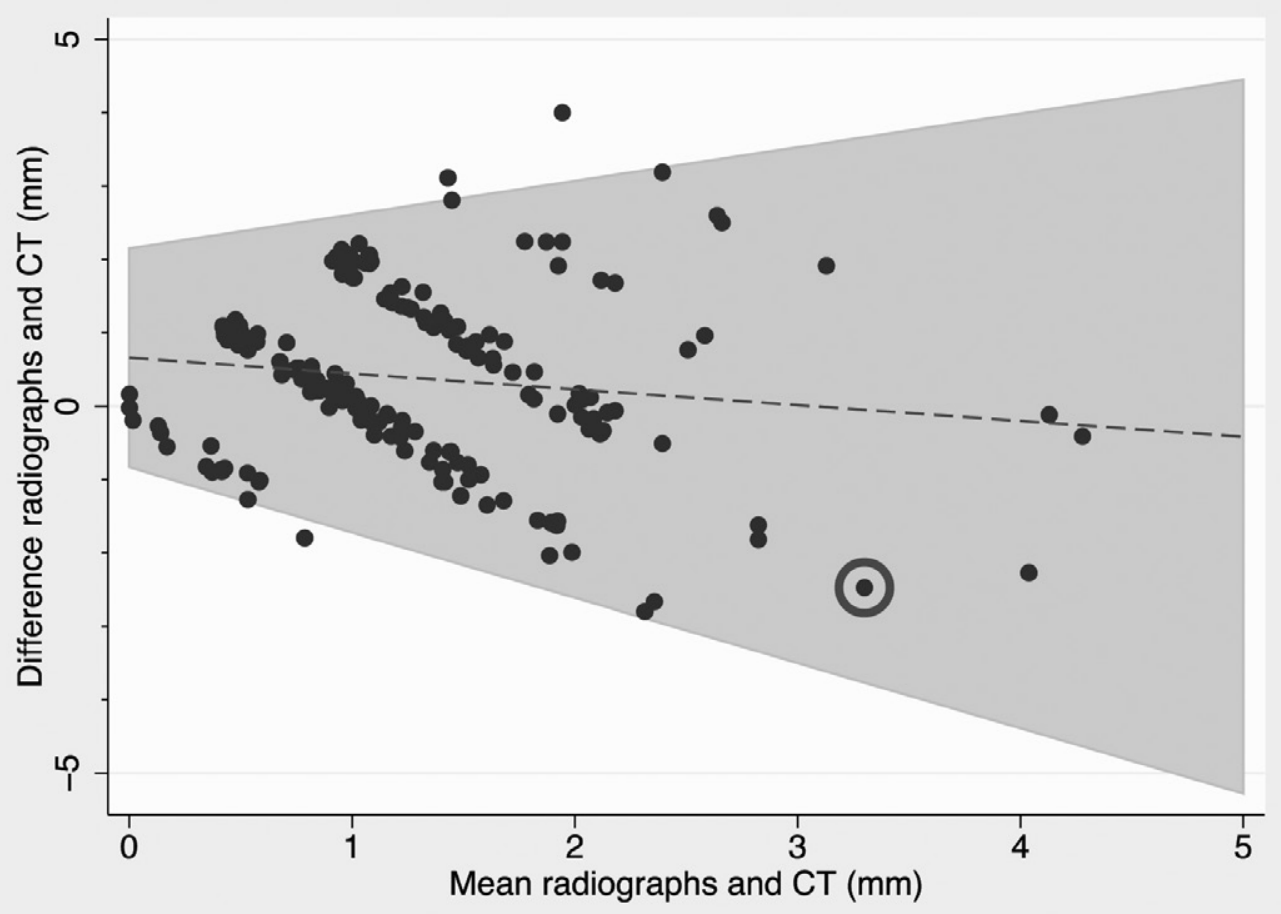

3A

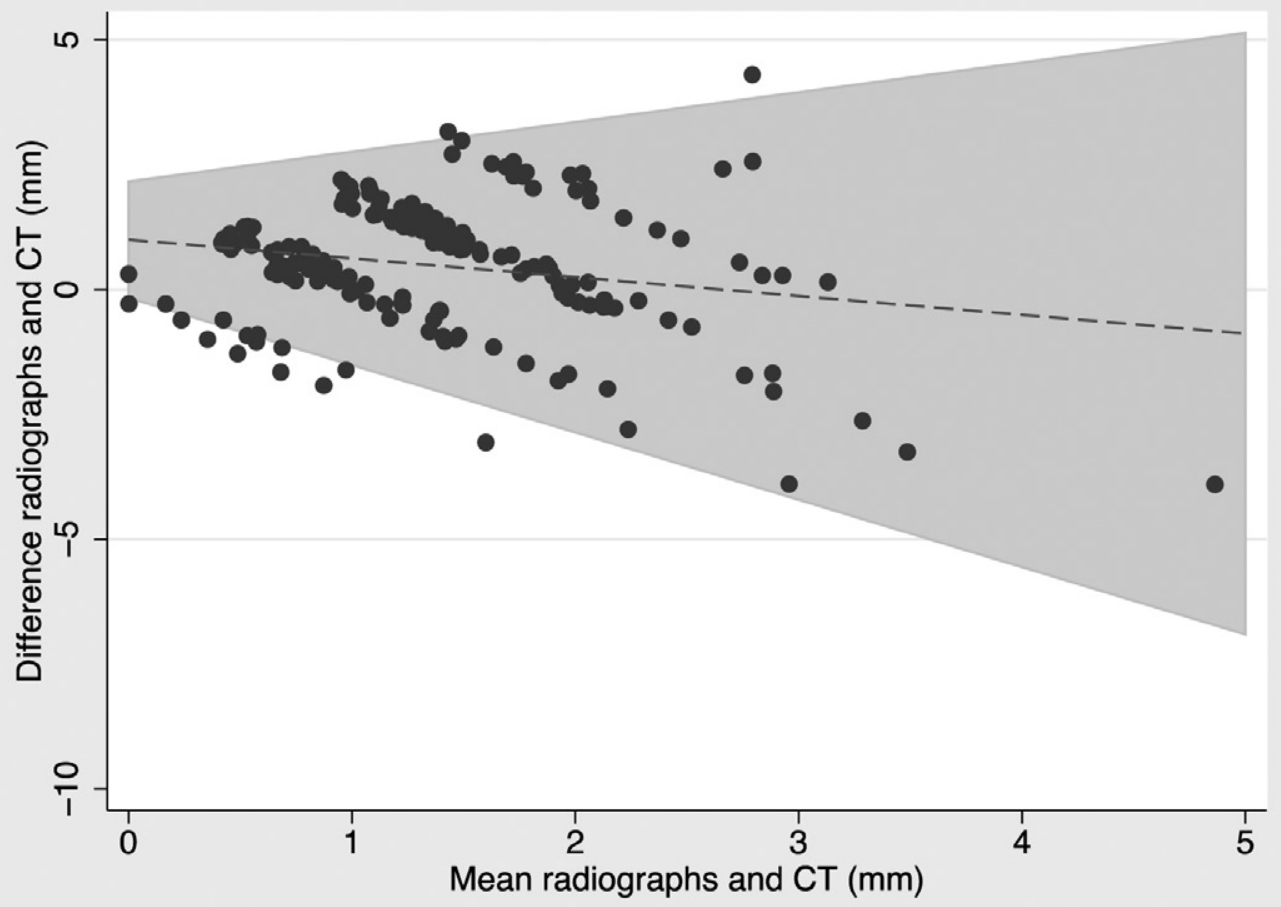




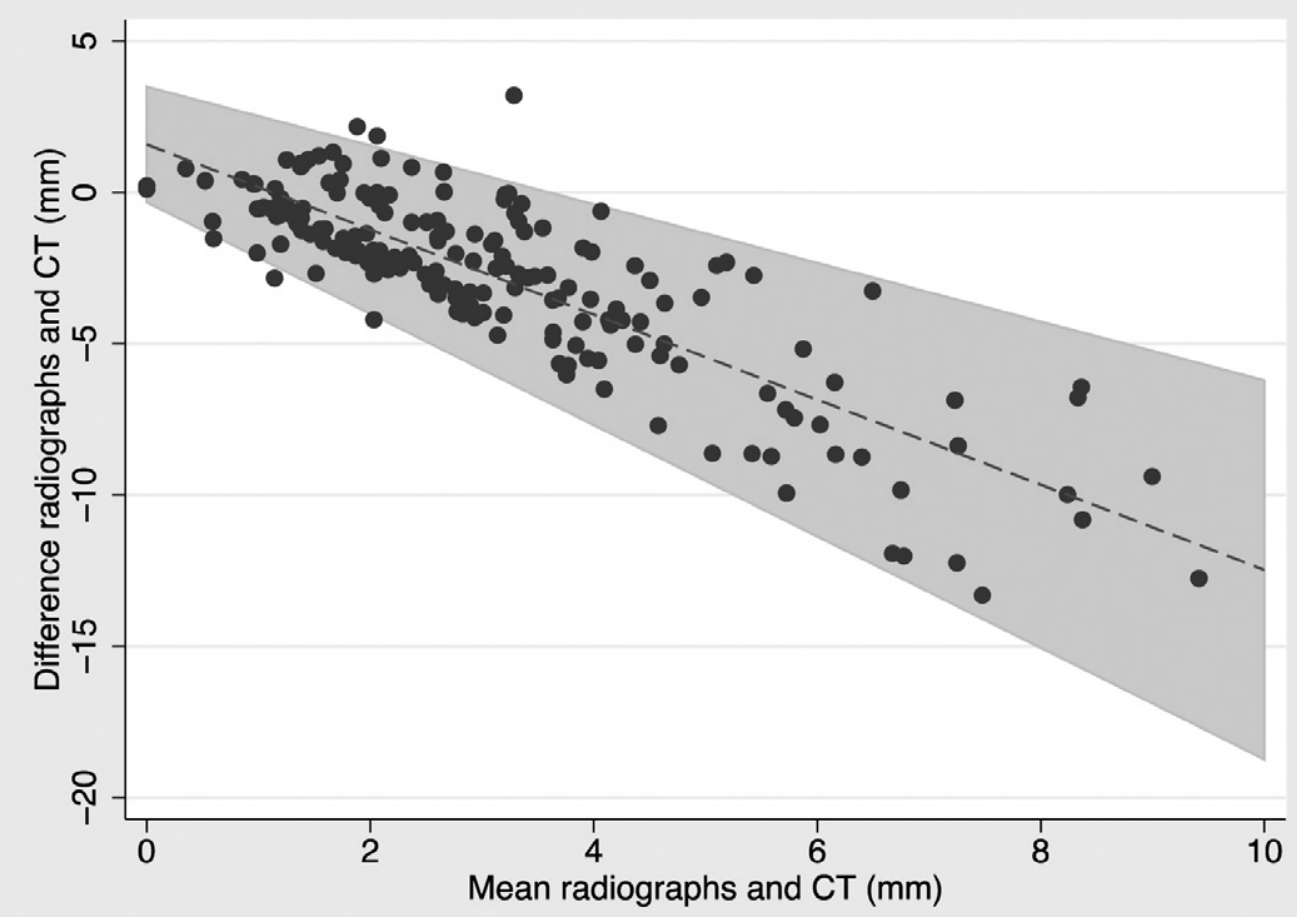

3c

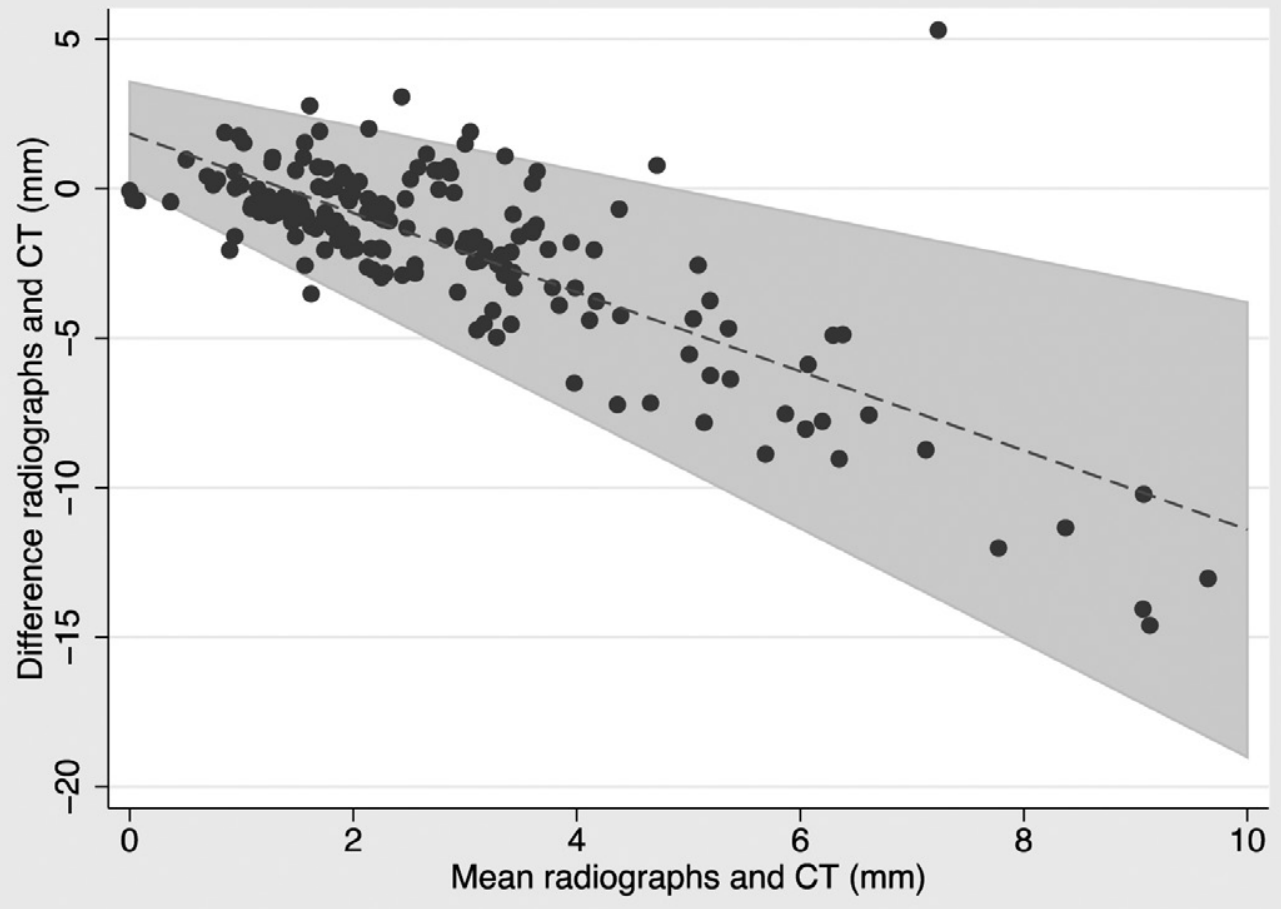




\section{APPENDIX 3: BLAND ALTMAN PLOTS OF GAP AND STEP OFF ON RADIOGRAPHS AND CT SCANS.}

Bland Altman plots assess systematic differences by plotting the mean of the radiograph and computed tomography measurement on the $\mathrm{x}$-axis and the difference between the two on the $\mathrm{y}$-axis. If there is perfect similarity, measurements will be plotted at zero on the $y$-axis. Each red dot resembles an individual patient. For example, patient $X$ (encircled dot in appendix 3A) had a radiographic step off of $2.1 \mathrm{~mm}$ and a CT step off of 4.6 (mean $=3.4$, difference $=-2.5$ ). The funnel shape in all plots indicates that when mean step off or gap increases, the difference between $\mathrm{CT}$ and radiographic measurements increases as well. The decreasing trend seen in gap deformity plots indicates that when the mean gap increases, CT consistently shows a larger gap than radiographs. 
CHAPTER

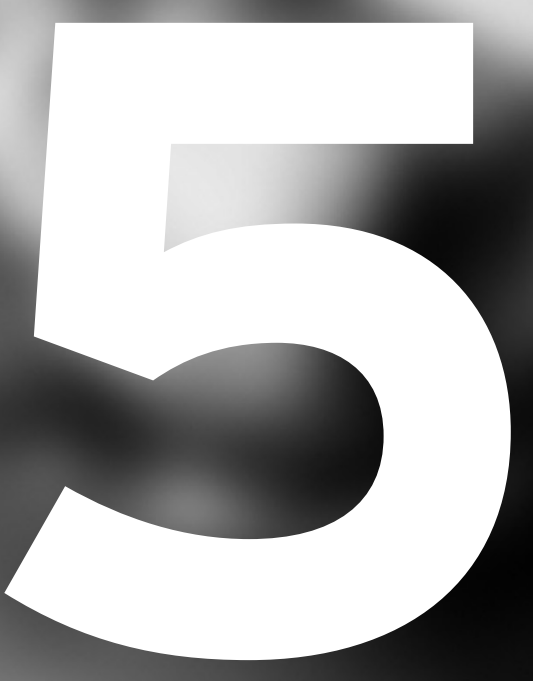




\title{
Are radiographic characteristics associated with outcome in
}

\section{surgically treated distal radius fractures?}

\author{
T. Teunis \\ D. Rikli \\ S. Meijer \\ W. van Leeuwen \\ A. Joeris \\ J. Jupiter
}

Submitted

Accepted presentation at the International Wrist Investigator's Workshop (2016).

Work supported by AO TK Trauma Network. 
Importance Evidence to date shows that in distal radius fractures radiographic measures don't correlate much with patient reported outcomes. This is counterintuitive to many and additional evidence is needed to change the way surgeons and patients think about treatment and prognosis.

Objectives To determine if there is an association between radiographic parameters after volar plating of distal radius fractures and change in disability, quality of life, range of motion, and grip strength after 12 weeks and 1 year.

Design Secondary use of data from a prospective cohort study.

Setting 6 hospitals in Switzerland and Germany.

Participants 67 patients with an AO/OTA type C fracture treated with a volar locking plate, of whom 59 (88\%) were available at 1 year. Mean age was 60 years (range 25 to 87 years), and 22 (33\%) were male.

Predictor variables In addition to patient demographics we recorded on post-operative PA radiographs: radial height, ulnarward inclination, ulnar variance, gap and step off. On lateral radiographs we measured: palmar tilt, scapholunate angle, teardrop angle, and anteroposterior distance. On CT scans we measured: gap (coronal, sagittal and axial) and step off (sagittal and coronal). In 3D models we measured: number of articular fragments, mean fragment articular surface area, 3D fragment displacement, and gap surface area.

Main Outcomes Change in PRWE, EQ5D, grip strength and wrist range of motion at 12 weeks and 1 year compared to pre-injury level.

Results Accounting for potential interaction of variables using multivariable analysis, smaller change in PRWE after 1 year was independently associated only with older age $(\beta-0.20,95 \% \mathrm{Cl}-0.39$ to -0.0034 , partial $\mathrm{R}^{2}$ 0.063, $P=0.046$ ). No variables were independently associated with change in EQ5D, wrist motion or grip strength 1 year after injury.

Conclusion In this cohort of limited displaced fractures, out of many radiographic fracture characteristics we found none to be associated with change in subjective and objective outcome 1 year after surgery. This should be considered when counseling patients on the risks and benefits of surgical treatment, especially when gross malalignment is absent and in older, low demand patients.

Level of Evidence Prognostic level I 


\section{INTRODUCTION}

Distal radius fractures nearly always heal, but they often heal with deformity. Patients are increasingly offered operative treatment prior to documented loss of reduction if the probability of healing with deformity is thought to be high with nonoperative treatment.' The correlation between deformity and subjective symptoms (e.g. DASH or PRWE scores) is limited. ${ }^{2}$ One cannot assume that deformity will cause problems for the patient, particularly in low energy injuries in low demand patients. This is illustrated by the different parameters recommended by national guidelines to define inadequate reduction and to consider surgery. ${ }^{3,4}$ Due to the limited strength of the recommendations both guidelines also recommend a strong incorporation of patient preferences. To complicate things further, some radiographic measures used to decide on treatment - in particularly step-off - have a low inter- and intra-rater reliability. ${ }^{5}$ Newly developed measures using Quantitative 3D computed tomography (Q3DCT), like 3D displacement and gap surface area, may be more accurate measures of displacement, and can be determined more reliably. ${ }^{6}$

We evaluated the association between radiographic parameters, including Q3DCT measures, after volar plating of distal radius fractures and change in disability, quality of life, range of motion, and grip strength after 12 weeks and 1 year.

\section{MATERIALS \& METHODS}

This study is a secondary analysis of a prospective multicenter trial (registered ClinicalTrials.gov, protocol number NCT01 103297). ${ }^{5}$ Institutional review board approval was obtained at each of the six participating sites. We included adult patients ( $\geq 18$ years) with a closed complete articular distal radius fracture (AO fracture type $\mathrm{Cl}$ to $\mathrm{C}^{7}$, confirmed by computed tomography) treated with a specific volar plate during our study period (2.4 mm VA LCP, Synthes, Oberdorf, Switzerland) ${ }^{8}$. Exclusion criteria were: (1) previous ipsilateral distal radius fractures or other current ipsilateral fractures (except the ulna), (2) pathologic fracture or active malignancy; (3) inability to complete enrollment forms due to any mental status or language problems; and (4) (potentially) pregnant or breastfeeding women. The surgical technique is described elsewhere ${ }^{9}$. Postoperatively patients in general started early active movement exercises, if necessary under the supervision of a hand therapist.

\section{Radiographic measurements}

We obtained radiographs and CT scans postoperatively (within 10 working days). On posteroanterior radiographs we measured: radial height, ulnarward inclination (also referred to as radial inclination), ulnar variance, gap, and step off. On lateral radiographs we recorded: palmar tilt, gap, step off, scapholunate angle, teardrop angle, and anteroposterior distance ${ }^{10,11}$. On CT we measured gap on sagittal, frontal and axial views and step off on sagittal and frontal views ${ }^{12,13}$. We created 3D fracture models and, using quantitative 3D CT techniques ${ }^{6,14}$. We measured: number of fragments, mean fragment surface area, surface area of the gap, and mean 3D fragment displacement (figure 1). By outlining the edges of the fracture gap in a 3D model, one can determine the surface area of the gap. In case of multiple gaps, the accumulated surface area was calculated. By measuring the amount of displacement on a fixed 3D grid on the $X, Y$ and $Z$-axis, one can calculate the 3D vector of displacement: 


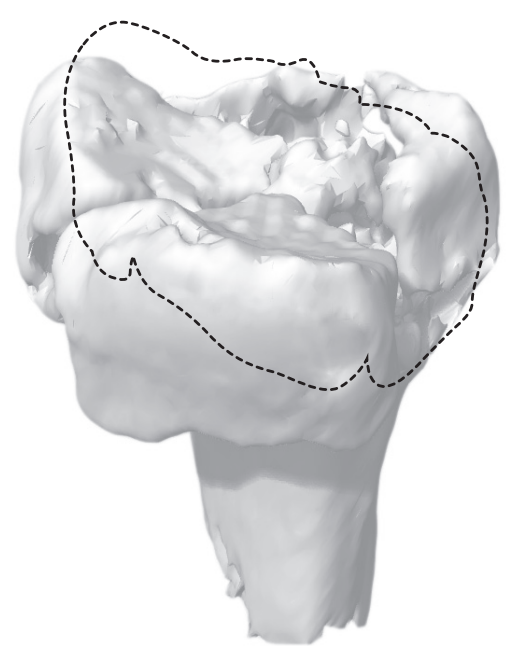

Unfractured distal radius

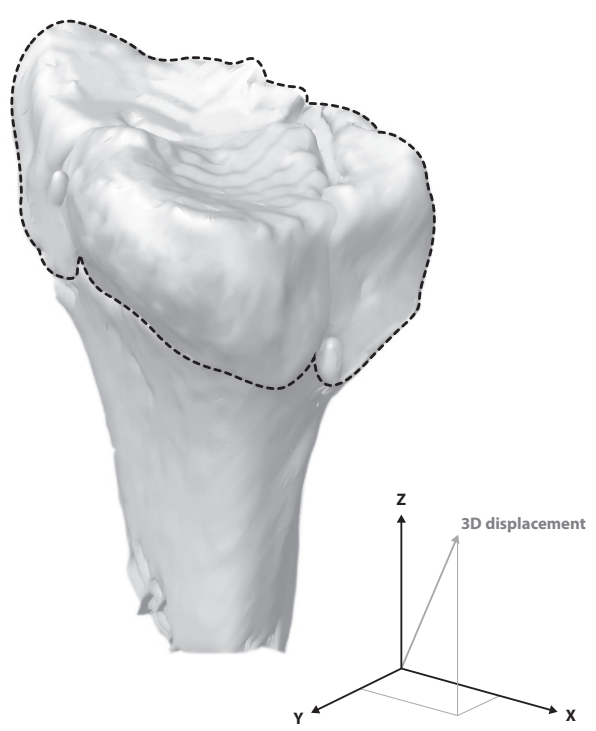

FIGURE 1.

A fractured radius is shown on the left, the fractured radial diaphysis is already positioned within that of the template (not shown). The fracture fragments are reduced within the unfractured template, shown on the right. The outline of the template's metaphysis is represented by the red dotted line. In the right lower corner the template's orientation is shown: $z$-axis represents proximal-distal displacement (loss of height), the $x$-axis radial-ulnar displacement, and the $y$-axis volar-dorsal displacement. Overall multidirectional (3D-) displacement is the vector of those axes.

Subsequently we calculated the mean 3D displacement of all fracture fragments. A video to depict our Q3DCT method is available at: http://www.traumaplatform.org/currentprojects. An independent radiologist assessed all radiographs; the primary author assessed all CT scans and Q3DCT measures. Previous study showed a high intra-class correlation (>0.82) for all radiographic and Q3DCT measurements, accept for posteroanterior stepoff (0.13) and gap (0.77), lateral step off (-0.036) and gap (0.17), scapholunate angle (0.58), teardrop angle (0.66), sagittal step off (0.56), axial gap (0.41), frontal step off $(0.17)$ and gap $(0.45) .^{5,6}$.

\section{Demographics and functional measurements}

Patients were enrolled before surgery. At this time we recorded: age, sex, body mass index, tobacco use, injury to the dominant side, mechanism of injury, AO classification, reduction status, and ulna fracture. Disability was assessed using the patient self-assessment of wrist function questionnaire (PRWE). PRWE score ranges from 0 to 100 , where higher scores indicated greater pain and disability ${ }^{15}$. We assessed health-related quality of life we using the EuroQoL5 (EQ5D). The EQ5D utility score was computed through a weighted regression-based algorithm, with a higher score indicating greater quality of life (eq5 d command, Stata 13.0, StataCorp LP, Texas, USA) ${ }^{16,17}$. Patients were interviewed regarding their upper limb function and quality of life as it was one week prior to the injury to determine a baseline.

Using a goniometer we measured wrist dorsal extension to palmar flexion, ulnar to radial deviation, and forearm supination to pronation. A composite value for the ROM was calculated by summing of the ranges of 
flexion, extension, radial and ulnar deviation and pronation and supination. We determined pre-injury range of motion by measuring motion of the unaffected side. Previous studies showed no difference in range of motion between the left and right hand in healthy adults. ${ }^{18,19}$

Grip strength was calculated as the average value of three successive measurements with a Jamar dynamometer. We estimated grip strength before injury from grip strength of the uninjured side. We assumed the right hand to be $10 \%$ stronger as compared to the left hand in right hand dominant patients. In left hand dominant patients we regarded grip strength to be equal between the left and the right side. ${ }^{20,21}$.

PRWE, EQ5D, wrist range of motion, and grip strength were also assessed at 12 weeks (range 8 to 15 weeks) and 12 months (range 11 to 13 months) after fracture. A site-specific research coordinator, not involved with patient care, obtained informed consent, administered the questionnaires, and performed the motion and grip measurements.

\section{Patient Demographics}

Between September 8, 2010 and January 22, 2013 we approach 81 consecutive patients to participate in our study. Fourteen patients were not eligible after surgery because they were treated with additional plates $(n=3)$ or a dorsal plate only $(n=4)$, because they sustained a partial articular fracture $(n=6)$, and because once accidently a different volar plate was used. Of the 67 remaining patients, 64 (96\%) were available a 12 weeks and 59 (88\%) were available at one year. Average age at enrollment was 60 ( \pm standard deviation [SD] 15) years, range 25 to 87 years; 22 (33\%) patients were male (Table 1).

\section{Table 1. Baseline characteristics}

Demographic variables

Enrolled

12 weeks

1 year

Age (range)

Male

Body mass index

Tobacco use

Mechanism of injury

Fall from standing height

Traffic accident

Other

Injured dominant side

A0 fracture classification

(1

(2

(3)

Ulna fracture

Closed reduction prior to surgery
Measurement

67

65 (97\%)

59 (88\%)

$60 \pm 15(25-87)$

$33 \%(22)$

$25 \pm 5.0$

$10 \%(7)$

$69 \%(46)$

$16 \%(11)$

$15 \%$ (10)

$42 \%$ (28)

$22 \%$ (15)

$30 \%(20)$

$48 \%$ (32)

$43 \%$ (29)

$45 \%$ (30)

Continuous data as mean \pm standard deviation, discrete data as percentage (number). 


\section{Statistical analysis}

We tested if change in function at 12 weeks and 1 year was associated with demographic and fracture characteristics (table 2). Measuring change from pre-injury levels attempted to eliminate the influence of any symptoms present from other previous hand problems. Continuous data is presented as mean $( \pm S D)$, discrete data as percentage and number. We used Pearson correlations to compare two continuous variables, two sided Student t-test for dichotomous and continuous data, and one-way analysis of variance for categorical and continuous data. The results of our bivariate analyses are reported in the Appendices (Appendix $1 \& 2$ ). We aimed to create a stepwise backwards multivariable linear regression model for each dependent variable associated with multiple independent variables on bivariate analysis with $P<0.10$. We considered $P<0.05$ significant.

Missing variables were imputed by mean imputation: grip at 1 year for 3 patients, ROM at 1 year for 1 patient, fragment's vector displacement in 2 patients.

Based on previous study ${ }^{22}$, a power analysis indicated that a sample size of 55 patients would provide $80 \%$ statistical power, with alpha set at 0.05 , assuming a model with five independent variables, where one radiological factor alone would account for $11 \%$ of the variability, assuming the complete model accounted for $25 \%$ of the variability.

\section{Table 2. Post-operative fracture characteristics and change in function}

\section{Radiographs}

\section{Posteroanterior}

- Radial height (mm)

- Ulnarward inclination $\left({ }^{\circ}\right)$

- Ulnar variance (mm)

- Step off (mm)

- Gap (mm)

\section{Lateral}

- Palmar tilt $\left({ }^{\circ}\right)$

$9.1 \pm 0.80$

- Scapholunate angle $\left({ }^{\circ}\right)$

$52 \pm 6.4$

- Teardrop angle $\left(^{\circ}\right)$

$66 \pm 3.7$

- Anteroposterior distance (mm)

- Step off (mm)

$17 \pm 0.72$

$1.6 \pm 0.68$

- Gap (mm)

\section{Computed tomography measures}

Axial gap (mm)

$2.2 \pm 1.1$

Sagittal

$$
\begin{array}{ll}
\text { - Step off (mm) } & 1.2 \pm 1.0 \\
\text { • Gap (mm) } & 3.5 \pm 2.4
\end{array}
$$

Frontal
- Step off (mm)
$1.2 \pm 0.90$
- Gap (mm)
$4.6 \pm 3.2$ 


$\begin{array}{ll}\text { Quantitative 3D computed tomography } & \\ \text { Number of fragments } & 3.8 \pm 1.6 \\ \text { Mean fragment surface area }\left(\mathrm{cm}^{2}\right) & 1.4 \pm 0.53 \\ \text { Surface area of gap }\left(\mathrm{cm}^{2}\right) & 0.48 \pm 0.54 \\ \text { 3D displacement }(\mathrm{mm}) & 1.5 \pm 1.4 \\ & \\ \text { Pre-injury function vs. } 12 \text { weeks after surgery } & \\ \text { Dorsal extension to palmar flexion }\left(^{\circ}\right) & -26 \pm 20 \\ \text { Ulnar to radial abduction }\left({ }^{\circ}\right) & -12 \pm 13 \\ \text { Pronation to supination }\left({ }^{\circ}\right) & -11 \pm 20 \\ \text { Composite range of motion } & -49 \pm 42 \\ \text { Grip strength (kg) } & -10 \pm 7.6 \\ \text { PRWE } & 18 \pm 20 \\ \text { EuroQoL5 } & -0.069 \pm 0.16 \\ \text { Pre-injury function vs. } 1 \text { year after surgery } & -18 \pm 32 \\ \text { Dorsal extension to palmar flexion }\left({ }^{\circ}\right) & \\ \text { Ulnar to radial deviation }\left({ }^{\circ}\right) & -3.3 \pm 9.7 \\ \text { Pronation to supination }\left({ }^{\circ}\right) & 5.6 \pm 12 \\ \text { Grip strength }(\mathrm{kg}) & -0.016 \pm 0.12\end{array}$

\section{Source of Funding}

The initial clinical investigation was performed with the support of the AO TK Trauma Network ${ }^{5}$.

\section{RESULTS}

Patient self-assessment of wrist function

Accounting for potential interaction of variables using multivariable analysis smaller increase in PRWE after 12 weeks was independently associated with greater ulnarward inclination $(\beta-5.9,95 \% \mathrm{Cl}-9.8$ to -1.9 , partial R $0.13, P=0.004$ ) (Adjusted $R^{2} 0.11$ ). In other words, on average each additional degree of ulnarward inclination after surgery resulted in 5.9 points less increase in PRWE, and thus a function closer to pre-injury level (table 3). Smaller increase in PRWE after 1 year was independently associated with older age ( $\beta$ regression coefficient [ $\beta$ ] -0.29 , 95\% confidence interval $[\mathrm{Cl}]-0.51$ to -0.072 , partial $\mathrm{R}^{2} 0.11, \mathrm{P}=0.01$ ) (Adjusted $\mathrm{R}^{2} 0.095$ ). In other words, on average for each extra year of age disability increased 0.29 points less. This means older people had a PRWE score closer to their pre-injury level than younger people. No radiological variables were associated with change PRWE at 1 year. 
Table 3. Multivariable analysis of factors associated with change in function after surgery

\begin{tabular}{|c|c|c|c|c|c|}
\hline Function at 12 weeks & $\begin{array}{l}\text { Regression coefficient } \\
\text { (95\% confidence interval) }\end{array}$ & Standard error & P value & Partial $\mathbf{R}^{2}$ & Adjusted R² \\
\hline \multicolumn{6}{|l|}{ Change in PRWE } \\
\hline Ulnarward inclination $\left({ }^{\circ}\right)$ & $-5.9(-9.8$ to -1.9$)$ & 2.0 & 0.004 & 0.13 & 0.11 \\
\hline \multicolumn{6}{|l|}{ Change in EuroQoL5 } \\
\hline Ulnarward inclination $\left({ }^{\circ}\right)$ & $0.045(0.013$ to 0.077$)$ & 0.016 & 0.006 & 0.12 & 0.10 \\
\hline \multicolumn{6}{|l|}{ Change in ROM } \\
\hline Scapholunate angle $\left({ }^{\circ}\right)$ & $-2.0(-3.6$ to -0.46$)$ & 0.79 & 0.012 & 0.11 & 0.18 \\
\hline 3D displacement (mm) & $-9.9(-18$ to -1.9$)$ & 4.0 & 0.017 & 0.097 & \\
\hline \multicolumn{6}{|l|}{ Change in grip strength } \\
\hline Male sex & $-5.1(-9.0$ to -1.1$)$ & 2.0 & 0.012 & 0.10 & 0.16 \\
\hline Mean fragment surface area $\left(\mathrm{mm}^{2}\right)$ & $4.8(1.4$ to 8.3$)$ & 1.7 & 0.007 & 0.12 & \\
\hline \multicolumn{6}{|l|}{ Function at 1 year } \\
\hline \multicolumn{6}{|l|}{ Change in PRWE } \\
\hline Age & $-0.29(-0.51$ to -0.072$)$ & 0.11 & 0.01 & 0.11 & 0.095 \\
\hline
\end{tabular}

\section{Quality of life}

After 12 weeks smaller decrease in EQ5D was independently associated with greater ulnarward inclination $\left(\beta 0.045,95 \% \mathrm{Cl} 0.013\right.$ to 0.077 , partial $\left.\mathrm{R}^{2} 0.12, \mathrm{P}=0.006\right)$ (Adjusted $\mathrm{R}^{2} 0.10$ ).

No variables were independently associated with change in EQ5D after 1 year.

\section{Composite range of motion}

Greater decrease in composite range of motion was independently associated with a greater scapholunate angle $\left(\beta-2.0,95 \% \mathrm{Cl}-3.6\right.$ to -0.46 , partial $\left.\mathrm{R}^{2} 0.11, \mathrm{P}=0.012\right)$ and greater $3 \mathrm{D}$ displacement $(\beta-9.9,95 \% \mathrm{Cl}-18$ to -1.9, partial $R^{2}$ 0.097, $P=0.017$ ) (Adjusted $R^{2} 0.18$ ).

No variables were independently associated with change in range of motion after 1 year.

\section{Grip strength}

Greater decrease in grip strength at 12 weeks was associated with male sex $(\beta-5.1,95 \% \mathrm{Cl}-9.0$ to -1.1 , partial $\left.R^{2} 0.10, P=0.012\right)$. Greater mean fragment surface area was associated with less decrease in grip strength $(\beta$ 4.8, 95\% Cl 1.4 to 8.3, partial $\mathrm{R}^{2} 0.12, \mathrm{P}=0.007$ ) (Adjusted $\mathrm{R}^{2}$ 0.16).

No variables were independently associated with change in grip strength after 1 year. 


\section{DISCUSSION}

The correlation between deformity and objective impairment and subjective symptoms is limited. ${ }^{2}$ The reasoning that radiographic deformity may not correlate with outcome is counterintuitive to many and additional evidence is needed to change the way surgeons and patients think about treatment and prognosis. We assessed if radiographic alignment of distal radius fractures was associated with functional outcome at 12 weeks and 1 year after volar plating.

This study has some limitations. First, in this surgically treated cohort only limited fracture displacement is to be expected. Our results may not apply to more grossly displaced fractures. Yet, the lack of any correlation at 1 year is striking. Secondly, osteoarthritic changes were not assessed due to only 1 year follow-up. Previous study showed symptomatic osteoarthritis to be uncommon after distal radius fracture. ${ }^{23}$ Thirdly, we imputed missing data by mean imputation, but this only comprised a small amount of data.

At 12 weeks greater ulnarward inclination was associated with less disability, but only explained $11 \%$ of the variation. At 1 year, older people more closely approach their pre-injury level of disability, and no radiographic factors were associated with PRWE. Comparing our results to studies using validated outcome questionnaires, one study looking at extra-articular non-operatively treated distal radius fractures found the presence of a third-party compensation claim, the level of education, and the presence of other medical problems to be associated with PRWE at 1 year. Palmar angulation, radial shortening, ulnarward inclination, and involvement of the ulna were not. ${ }^{24}$ Another study assessing PRWE at a mean of 29 months (range 10 to 46) after k-wire fixation found only Frykman type fractures VII and VIII to be associated with worse disability; again palmar angulation, radial shortening, and ulnarward inclination were not. ${ }^{25} \mathrm{~A}$ study assessing PRWE at 1 year after k-wire fixation found greater radial shortening was associated with more disability. However, this study did not use multivariable analysis to assess independent association. ${ }^{26}$ Our results and most of the previous literature suggest radiographic alignment is only of limited predictive value for disability at 1 year.

Twelve weeks after surgery people with greater ulnarward inclination were closer to their pre-injury quality of life. After 1 year no factors were associated with changes in quality of life. In a cohort of non-operatively treated patients over 50 years old with a distal radius fracture Medical Outcomes Study Short-Form 12 at 6 months was no different between patients achieving 'acceptable' or 'unacceptable' palmar tilt, ulnarward inclination, radial height, ulnar variance, or intra-articular step-off and gap. ${ }^{27}$ Fracture alignment seems not associated with quality of life at 1 year.

At twelve weeks greater decrease in composite range of motion was associated with greater scapholunate angle and 3D displacement, but no variables were associated with range of motion at 1 year. Previous study found that scapolunate angle has a low interobserver reliability (intra-class correlation 0.58 ), limiting the relevance of this measure. ${ }^{5}$ One study found that at a mean of 38 years after non-operatively treated fractures composite motion on average was reduced to $92 \%$ of the uninjured side if a step-off was present, compared to $98 \%$ when there was no step. This was mainly caused by a $10^{\circ}$ loss of palmar flexion. ${ }^{23}$ Conversely in our study, step-off was not associated with range of motion. Step-off also has a low interobserver reliability. ${ }^{5}$ In our study, at 1 year, the influence of fracture alignment on range of motion is limited.

A greater decrease in grip strength at twelve weeks was associated with male sex and smaller fragment surface area (i.e., greater communition at the articular surface). Again, no variables were associated with change in grip strength at 1 year. Two other studies also found no association between grip strength and radiographic parameters more than 1 year after fracture fixation. ${ }^{26,28}$ One study of non-operatively treated distal radius 
fracture found that more than $20^{\circ}$ of dorsal tilt reduced grip strength to $89 \%$ of the uninjured at a mean of 38 years after fracture. ${ }^{23}$ In our cohort no patients had residual dorsal tilt, potentially explaining the lack of correlation in our study.

In this cohort of limited displaced fractures, a couple of radiographic measures were associated with early recovery, but no measure is associated with symptoms or impairment at 1 year after fracture. This should be considered when counseling patients on the risks and benefits of surgical treatment, especially when gross malalignment is absent and in older, low demand patients. The variation seen in recovery between patients might be related to other factors such as the tendency to misinterpret or overinterpret nociception (i.e., catastrophic thinking), heightened concern about illness, and social and cultural factors on illness behavior. ${ }^{29}$ There seems to be limited value to Q3DCT measures in distal radius fractures. 


\section{REFERENCES}

1. Chung KC, Shauver MJ, Birkmeyer JD. Trends in the United States in the treatment of distal radial fractures in the elderly. J Bone Joint Surg Am. 2009;91(8):1868-1873.

2. Souer JS, Lozano-Calderon SA, Ring D. Predictors of wrist function and health status after operative treatment of fractures of the distal radius. J Hand Surg Am. 2008;33(2):157-163.

3. American Academy of Orthopaedic Surgeons. The treatment of distal radius fractures: guidelines and evidence report. 2009.

4. Nederlandse Vereniging voor Heelkunde. Richtlijn distale radius fracturen: diagnostiek en behandeling. 2010.

5. Teunis T, Joeris A, Schaser KD, et al. Evaluation of radiographic fracture position one year after variable angle locking volar distal radius plating. A prospective multicenter case series. J Hand Surg Eur Vol. 2016;Accepted.

6. Teunis T, Bosma NH, Lubberts B, Ter Meulen DP, Ring D. Melone's concept revisited: 3D quantification of fragment displacement. . J Hand Microsurg. 2016;Accepted.

7. Classification OTACfCa. Fracture and dislocation compendium. Orthopaedic Trauma Association Committee for Coding and Classification. J.Orthop.Trauma. 1996;10 Suppl 1: 154 pages.

8. Synthes. Variable Angle LCP Two-Column Volar Distal Radius Plate 2.4. For fragment-specific fracture fixation with variable angle locking technology. 2015; http://synthes.vo.Ilnwd.net/o16/LLNWMB8/INT Mobile/Synthes International/Product Support Material/legacy_Synthes_PDF/DSEM-TRM-0815-0464_LR.pdf. Accessed October $17,2015$.

9. Jupiter JB, Marent-Huber M, Group LCPS. Operative management of distal radial fractures with 2.4-millimeter locking plates. A multicenter prospective case series. J Bone Joint Surg Am. 2009;91(1):55-65.

10. Kreder HJ, Hanel DP, McKee M, Jupiter J, McGillivary G, Swiontkowski MF. X-ray film measurements for healed distal radius fractures. J Hand Surg Am. 1996;21(1):31-39.

11. Medoff RJ. Essential radiographic evaluation for distal radius fractures. Hand Clin. 2005;21(3):279-288.

12. Cole RJ, Bindra RR, Evanoff BA, Gilula LA, Yamaguchi K, Gelberman RH. Radiographic evaluation of osseous displacement following intra-articular fractures of the distal radius: reliability of plain radiography versus computed tomography. J Hand Surg Am. 1997;22(5):792-800.

13. Arora S, Grover SB, Batra S, Sharma VK. Comparative evaluation of postreduction intra-articular distal radial fractures by radiographs and multidetector computed tomography. J Bone Joint Surg Am. 2010;92(15):2523-2532.

14. Teunis T, Bosma NH, Lubberts B, Ter Meulen DP, Ring D. MM Q3DCT Radius. 2014; http://youtu.be/MOniRAoZ2xw Accessed 25 March, 2014.

15. MacDermid JC, Turgeon T, Richards RS, Beadle M, Roth JH. Patient rating of wrist pain and disability: a reliable and valid measurement tool. J Orthop Trauma. 1998;12(8):577-586.

16. Ramos-Goñi JM, Rivero-Arias O. eq5d: A command to calculate index values for the EQ-5D quality-of-life instrument. Stata Journal. 2011;11(1):120-125.

17. EuroQol G. EuroQol--a new facility for the measurement of health-related quality of life. Health Policy. 1990;16(3):199208.

18. Kitsoulis P, Paraskevas G, Iliou K, Kanavaros P, Marini A. Clinical study of the factors affecting radioulnar deviation of the wrist joint. BMC Musculoskelet Disord. 2010;11:9.

19. Smahel Z, Klimova A. The effect of age and exercise on wrist mobility. Acta Chir Plast. 2005;47(3):92-97.

20. Crosby CA, Wehbe MA, Mawr B. Hand strength: normative values. J Hand Surg Am. 1994;19(4):665-670.

21. Petersen P, Petrick M, Connor H, Conklin D. Grip strength and hand dominance: challenging the $10 \%$ rule. Am J Occup Ther. 1989;43(7):444-447.

22. Teunis T, Stoop N, Park CJ, Ring D. What factors are associated with a second opioid prescription after treatment of distal radius fractures with a volar locking plate? HAND. 2015; published online.

23. Forward DP, Davis TR, Sithole JS. Do young patients with malunited fractures of the distal radius inevitably develop symptomatic post-traumatic osteoarthritis? J Bone Joint Surg Br. 2008;90(5):629-637.

24. Grewal R, MacDermid JC, Pope J, Chesworth BM. Baseline predictors of pain and disability one year following extraarticular distal radius fractures. Hand (N Y). 2007;2(3):104-111.

25. Barton T, Chambers C, Bannister C. A comparison between subjective outcome score and moderate radial shortening following a fractured distal radius in patients of mean age 69 years. J Hand Surg Eur Vol. 2007;32(2):165-169.

26. Karnezis IA, Panagiotopoulos E, Tyllianakis M, Megas P, Lambiris E. Correlation between radiological parameters and patient-rated wrist dysfunction following fractures of the distal radius. Injury. 2005;36(12):1435-1439.

27. Jaremko JL, Lambert RG, Rowe BH, Johnson JA, Majumdar SR. Do radiographic indices of distal radius fracture reduction predict outcomes in older adults receiving conservative treatment? Clin Radiol. 2007;62(1):65-72.

28. Catalano LW, 3rd, Cole RJ, Gelberman RH, Evanoff BA, Gilula LA, Borrelli J, Jr. Displaced intra-articular fractures of the distal aspect of the radius. Long-term results in young adults after open reduction and internal fixation. J Bone Joint Surg Am. 1997;79(9):1290-1302.

29. Teunis T, Bot AG, Thornton ER, Ring D. Catastrophic Thinking is Associated with Finger Stiffness After Distal Radius Fracture Surgery. J Orthop Trauma. 2015. 
Appendix 1. Bivariate analysis of explanatory variables and change in function between pre-injury and 12 weeks after surgery

\begin{tabular}{|c|c|c|c|c|c|c|c|c|}
\hline Radiographs & PRWE & $P$ value & EuroQoL5 & Pvalue & Composite ROM & Pvalue & Grip strength & $P$ value \\
\hline \multicolumn{9}{|l|}{ Posteroanterior } \\
\hline • Radial height (mm) & -0.22 & 0.081 & 0.19 & 0.14 & 0.092 & 0.48 & -0.00010 & 0.99 \\
\hline - Ulnarward inclincation $\left(^{\circ}\right)$ & -0.35 & 0.004 & 0.34 & 0.0061 & 0.11 & 0.39 & 0.14 & 0.27 \\
\hline - Ulnar variance (mm) & 0.021 & 0.87 & -0.0020 & 0.99 & -0.064 & 0.63 & 0.028 & 0.83 \\
\hline - Step off (mm) & -0.011 & 0.93 & 0.053 & 0.68 & -0.27 & 0.034 & -0.16 & 0.20 \\
\hline - Gap (mm) & 0.017 & 0.90 & 0.0050 & 0.97 & -0.24 & 0.070 & -0.17 & 0.19 \\
\hline \multicolumn{9}{|l|}{ Lateral } \\
\hline - Palmar tilt $\left({ }^{\circ}\right)$ & -0.12 & 0.34 & 0.096 & 0.45 & 0.042 & 0.75 & 0.045 & 0.73 \\
\hline - Scapholunate angle $\left({ }^{\circ}\right)$ & 0.050 & 0.70 & -0.10 & 0.43 & -0.35 & 0.005 & -0.12 & 0.34 \\
\hline - Teardrop angle $\left({ }^{\circ}\right)$ & 0.049 & 0.70 & -0.26 & 0.039 & -0.075 & 0.57 & -0.10 & 0.41 \\
\hline - Anteroposterior distance (mm) & -0.26 & 0.038 & 0.11 & 0.38 & 0.011 & 0.93 & 0.14 & 0.28 \\
\hline -Step off (mm) & 0.032 & 0.80 & 0.069 & 0.59 & -0.15 & 0.25 & 0.044 & 0.73 \\
\hline - Gap (mm) & 0.058 & 0.65 & 0.0030 & 0.98 & -0.12 & 0.37 & -0.0018 & 0.99 \\
\hline \multicolumn{9}{|l|}{ CT } \\
\hline Axial gap (mm) & -0.021 & 0.87 & -0.025 & 0.85 & -0.063 & 0.63 & -0.10 & 0.42 \\
\hline \multicolumn{9}{|l|}{ Sagittal } \\
\hline - Step off (mm) & 0.024 & 0.86 & 0.025 & 0.85 & -0.10 & 0.44 & -0.023 & 0.86 \\
\hline - Gap (mm) & -0.043 & 0.74 & 0.12 & 0.35 & 0.021 & 0.87 & -0.16 & 0.22 \\
\hline \multicolumn{9}{|l|}{ Frontal } \\
\hline - Step off (mm) & -0.25 & 0.058 & 0.16 & 0.23 & 0.13 & 0.32 & 0.048 & 0.72 \\
\hline - Gap (mm) & -0.087 & 0.51 & 0.098 & 0.46 & -0.041 & 0.76 & -0.018 & 0.90 \\
\hline \multicolumn{9}{|l|}{ Quantitative 3D CT } \\
\hline Number of fragments & 0.040 & 0.76 & 0.11 & 0.40 & -0.045 & 0.74 & -0.33 & 0.0079 \\
\hline Mean fragment surface area $\left(\mathrm{mm}^{2}\right)$ & -0.088 & 0.49 & -0.046 & 0.72 & 0.033 & 0.80 & 0.31 & 0.013 \\
\hline Surface area of gap (mm²) & 0.071 & 0.58 & -0.0007 & 1.0 & -0.012 & 0.92 & -0.20 & 0.11 \\
\hline 3D displacement (mm) & 0.14 & 0.26 & -0.023 & 0.85 & -0.34 & 0.0076 & -0.15 & 0.22 \\
\hline \multicolumn{9}{|l|}{ Demographics } \\
\hline Age (range) & 0.16 & 0.21 & 0.039 & 0.76 & 0.14 & 0.30 & -0.033 & 0.80 \\
\hline \multicolumn{9}{|l|}{ Sex } \\
\hline • Male & $15 \pm 18$ & 0.48 & $-0.065 \pm 0.14$ & 0.94 & $-40 \pm 38$ & 0.27 & $-13 \pm 8.1$ & 0.021 \\
\hline • Female & $19 \pm 22$ & & $-0.068 \pm 0.18$ & & $-53 \pm 44$ & & $-8.7 \pm 7.3$ & \\
\hline Body mass index & -0.028 & 0.83 & 0.032 & 0.80 & -0.043 & 0.75 & -0.083 & 0.51 \\
\hline \multicolumn{9}{|l|}{ Tobacco use } \\
\hline • Yes & $20 \pm 18$ & 0.76 & $-0.049 \pm 0.075$ & 0.77 & $-38 \pm 17$ & 0.49 & $-7.3 \pm 9.4$ & 0.33 \\
\hline • No & $18 \pm 21$ & & $-0.069 \pm 0.17$ & & $-50 \pm 44$ & & $-11 \pm 7.7$ & \\
\hline
\end{tabular}


Mechanism of injury

- Fall from standing height

$\begin{array}{lll}20 \pm 22 & 0.52 & 0.83 \pm 0.20 \\ 13 \pm 13 & & 0.88 \pm 0.14 \\ 15 \pm 20 & 0.96 \pm 0.10\end{array}$

0.11

$-51 \pm 40$

$0.87 \quad-9.2 \pm 6.4$

0.21

- Traffic accident

$15 \pm 20$

$-43 \pm 62$

$-14 \pm 8.7$

- Other

$16 \pm 18$

0.59

$-0.041 \pm 0.15$

0.27

$-47 \pm 50$

0.67

$-12 \pm 7.6$

0.24

- No

$19 \pm 22$

$-0.087 \pm 0.17$

$-51 \pm 35$

$-9.2 \pm 7.9$

A0 fracture classication

- C1

$19 \pm 20 \quad 0.63$

$0.84 \pm 0.13$

0.55

$-46 \pm 29$

0.72

$-9.7 \pm 6.9$

0.96

$\cdot \mathrm{C2}$

$21 \pm 25$

$0.83 \pm 0.21$

$-56 \pm 60$

$-10 \pm 7.7$

. C3

$15 \pm 17$

$0.88 \pm 0.18$

$-46 \pm 33$

$-10 \pm 8.5$

Ulna fracture

- Yes

$15 \pm 21 \quad 0.27$

$-0.064 \pm 0.18$

0.87

$-49 \pm 37$

0.98

$-11 \pm 6.3$

0.48

- No

$20 \pm 20$

$-0.071 \pm 0.15$

$-49 \pm 47$

$-9.6 \pm 8.9$

Closed reduction prior to surgery

$$
\text { - Yes }
$$

$18 \pm 21 \quad 0.96$

$-0.095 \pm 0.18$

0.24

$-48 \pm 39$

0.81

$-9.2 \pm 8.8$

0.36

- No

$18 \pm 20$

$-0.046 \pm 0.15$

$-50 \pm 46$

$-11 \pm 7.0$

$P R W E=$ Patient self-assessment of wrist function questionnaire; $R O M=$ range of motion; $P$ value $<0.05$ indicates significant difference. 


\section{Appendix 2. Bivariate analysis of explanatory variables and change in function between pre-injury and 1 year after surgery}

\begin{tabular}{|c|c|c|c|c|c|c|c|c|}
\hline Radiographs & PRWE & Pvalue & EuroQoL5 & $P$ value & Composite ROM & $P$ value & Grip strength & $P$ value \\
\hline \multicolumn{9}{|l|}{ Posteroanterior } \\
\hline • Radial height (mm) & -0.078 & 0.55 & -0.14 & 0.30 & -0.034 & 0.81 & 0.16 & 0.23 \\
\hline - Ulnarward inclincation $\left(^{\circ}\right)$ & -0.086 & 0.52 & -0.071 & 0.59 & 0.12 & 0.39 & 0.070 & 0.60 \\
\hline - Ulnar variance (mm) & -0.061 & 0.64 & 0.13 & 0.32 & -0.074 & 0.59 & 0.016 & 0.90 \\
\hline - Step off (mm) & -0.088 & 0.51 & 0.10 & 0.45 & -0.18 & 0.18 & -0.22 & 0.087 \\
\hline - Gap (mm) & -0.073 & 0.58 & 0.16 & 0.23 & 0.020 & 0.88 & -0.0087 & 0.95 \\
\hline \multicolumn{9}{|l|}{ Lateral } \\
\hline - Palmar tilt $\left(^{\circ}\right)$ & -0.0064 & 0.96 & -0.045 & 0.74 & 0.065 & 0.64 & 0.075 & 0.57 \\
\hline - Scapholunate angle $\left({ }^{\circ}\right)$ & -0.041 & 0.76 & 0.10 & 0.44 & -0.0056 & 0.97 & 0.047 & 0.72 \\
\hline - Teardrop angle $\left({ }^{\circ}\right)$ & 0.047 & 0.72 & -0.18 & 0.18 & 0.071 & 0.61 & -0.031 & 0.82 \\
\hline - Anteroposterior distance (mm) & -0.17 & 0.20 & -0.10 & 0.43 & 0.055 & 0.69 & 0.063 & 0.64 \\
\hline - Step off (mm) & 0.19 & 0.13 & 0.067 & 0.62 & -0.15 & 0.27 & 0.013 & 0.92 \\
\hline - Gap (mm) & 0.19 & 0.15 & 0.13 & 0.31 & 0.031 & 0.82 & 0.067 & 0.62 \\
\hline \multicolumn{9}{|l|}{ СТ } \\
\hline Axial gap (mm) & -0.052 & 0.70 & 0.066 & 0.62 & -0.18 & 0.17 & -0.044 & 0.74 \\
\hline \multicolumn{9}{|l|}{ Sagittal } \\
\hline - Step off (mm) & -0.060 & 0.67 & -0.038 & 0.78 & -0.11 & 0.41 & -0.054 & 0.70 \\
\hline - Gap (mm) & 0.018 & 0.89 & 0.075 & 0.59 & -0.066 & 0.63 & -0.17 & 0.21 \\
\hline \multicolumn{9}{|l|}{ Frontal } \\
\hline - Step off (mm) & -0.014 & 0.92 & -0.018 & 0.90 & -0.035 & 0.80 & -0.18 & 0.19 \\
\hline - Gap (mm) & -0.065 & 0.64 & -0.0056 & 0.97 & -0.086 & 0.53 & -0.082 & 0.55 \\
\hline \multicolumn{9}{|l|}{ Quantitative 3D CT } \\
\hline Number of fragments & -0.073 & 0.59 & 0.20 & 0.12 & -0.084 & 0.54 & -0.12 & 0.38 \\
\hline Mean fragment surface area $\left(\mathrm{mm}^{2}\right)$ & -0.16 & 0.22 & -0.046 & 0.73 & 0.023 & 0.86 & 0.24 & 0.067 \\
\hline Surface area of gap $\left(\mathrm{mm}^{2}\right)$ & -0.0046 & 0.97 & -0.077 & 0.56 & -0.040 & 0.77 & -0.10 & 0.43 \\
\hline 3D displacement (mm) & -0.014 & 0.92 & 0.055 & 0.68 & -0.13 & 0.35 & -0.088 & 0.51 \\
\hline \multicolumn{9}{|l|}{ Demographics } \\
\hline Age (range) & -0.33 & 0.010 & -0.022 & 0.86 & 0.21 & 0.12 & -0.037 & 0.78 \\
\hline \multicolumn{9}{|l|}{ Sex } \\
\hline - Male & $3.4 \pm 6.4$ & 0.24 & $-0.018 \pm 0.086$ & 0.98 & $-17 \pm 30$ & 0.96 & $-2.9 \pm 14$ & 0.82 \\
\hline - Female & $7.5 \pm 15$ & & $-0.017 \pm 0.15$ & & $-17 \pm 33$ & & $-3.5 \pm 7.7$ & \\
\hline Body mass index & 0.10 & 0.44 & -0.11 & 0.41 & -0.13 & 0.36 & -0.047 & 0.72 \\
\hline \multicolumn{9}{|l|}{ Tobacco use } \\
\hline - Yes & $16 \pm 18$ & 0.025 & $-0.027 \pm 0.13$ & 0.84 & $-3.7 \pm 38$ & 0.24 & $-6.2 \pm 5.6$ & 0.43 \\
\hline • No & $4.8 \pm 11$ & & $-0.016 \pm 0.052$ & & $-19 \pm 30$ & & $-2.9 \pm 11$ & \\
\hline \multicolumn{9}{|l|}{ Mechanism of injury } \\
\hline Fall from standing height & $6.1 \pm 14$ & 0.82 & $-0.026 \pm 0.14$ & 0.73 & $-15 \pm 31$ & 0.77 & $-3.6 \pm 7.0$ & 0.64 \\
\hline Traffic accident & $4.3 \pm 7.7$ & & $-0.0029 \pm 0.13$ & & $-19 \pm 43$ & & $-0.63 \pm 19$ & \\
\hline Other & $7.8 \pm 8.2$ & & $0.0054 \pm 0.84$ & & $-23 \pm 20$ & & $-4.8 \pm 9.0$ & \\
\hline \multicolumn{9}{|l|}{ Injured dominant side } \\
\hline Yes & $5.0 \pm 15$ & 0.54 & $-0.0091 \pm 0.13$ & 0.66 & $-9.3 \pm 35$ & 0.075 & $-4.4 \pm 7.1$ & 0.45 \\
\hline No & $7.0 \pm 11$ & & $-0.024 \pm 0.13$ & & $-24 \pm 4.8$ & & $-2.4 \pm 12$ & \\
\hline \multicolumn{9}{|l|}{ A0 fracture classication } \\
\hline C1 & $7.0 \pm 21$ & 0.90 & $-0.029 \pm 0.17$ & 0.52 & $-30 \pm 27$ & 0.083 & $-2.2 \pm 7.5$ & 0.80 \\
\hline C2 & $4.9 \pm 7.8$ & & $-0.043 \pm 0.090$ & & $-2.5 \pm 41$ & & $-4.7 \pm 5.1$ & \\
\hline C 3 & $6.3 \pm 9.2$ & & $0.0013 \pm 0.12$ & & $-20 \pm 26$ & & $-3.1 \pm 13$ & \\
\hline \multicolumn{9}{|l|}{ Ulna fracture } \\
\hline Yes & $4.2 \pm 12$ & 0.28 & $-0.036 \pm 0.14$ & 0.30 & $7.0 \pm 35$ & 0.97 & $-2.3 \pm 13$ & 0.40 \\
\hline No & $7.7 \pm 13$ & & $-0.0011 \pm 0.11$ & & $5.2 \pm 29$ & & $-4.5 \pm 6.2$ & \\
\hline \multicolumn{9}{|l|}{ Closed reduction prior to surgery } \\
\hline Yes & $7.0 \pm 16$ & 0.62 & $-0.029 \pm 0.12$ & 0.53 & $5.1 \pm 26$ & 0.18 & $-3.0 \pm 15$ & 0.87 \\
\hline No & $5.4 \pm 9.8$ & & $-0.0080 \pm 0.14$ & & $6.3 \pm 35$ & & $-3.5 \pm 5.2$ & \\
\hline
\end{tabular}

$P R W E=$ Patient self-assessment of wrist function questionnaire; $R O M=$ range of motion; $P$ value $<0.05$ indicates significant difference. 
PART

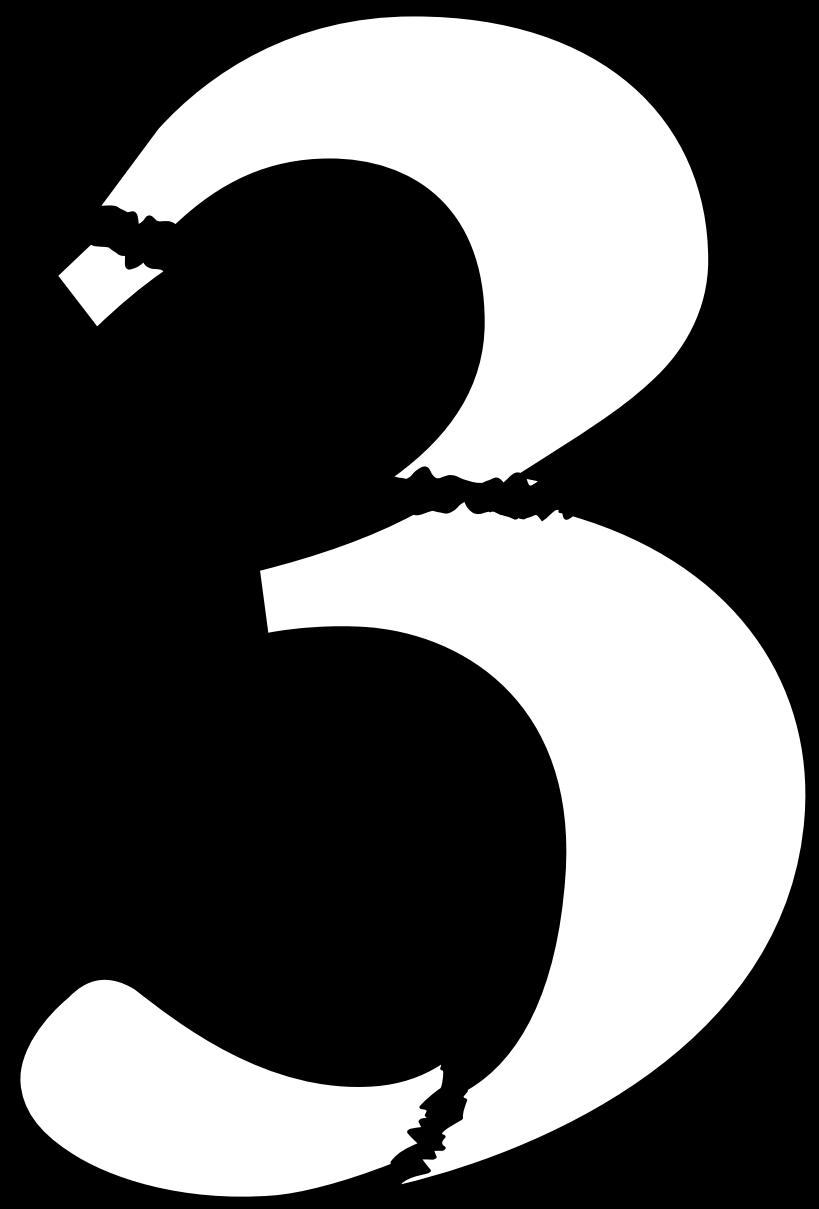


Aspects of recovery 
CHAPTER

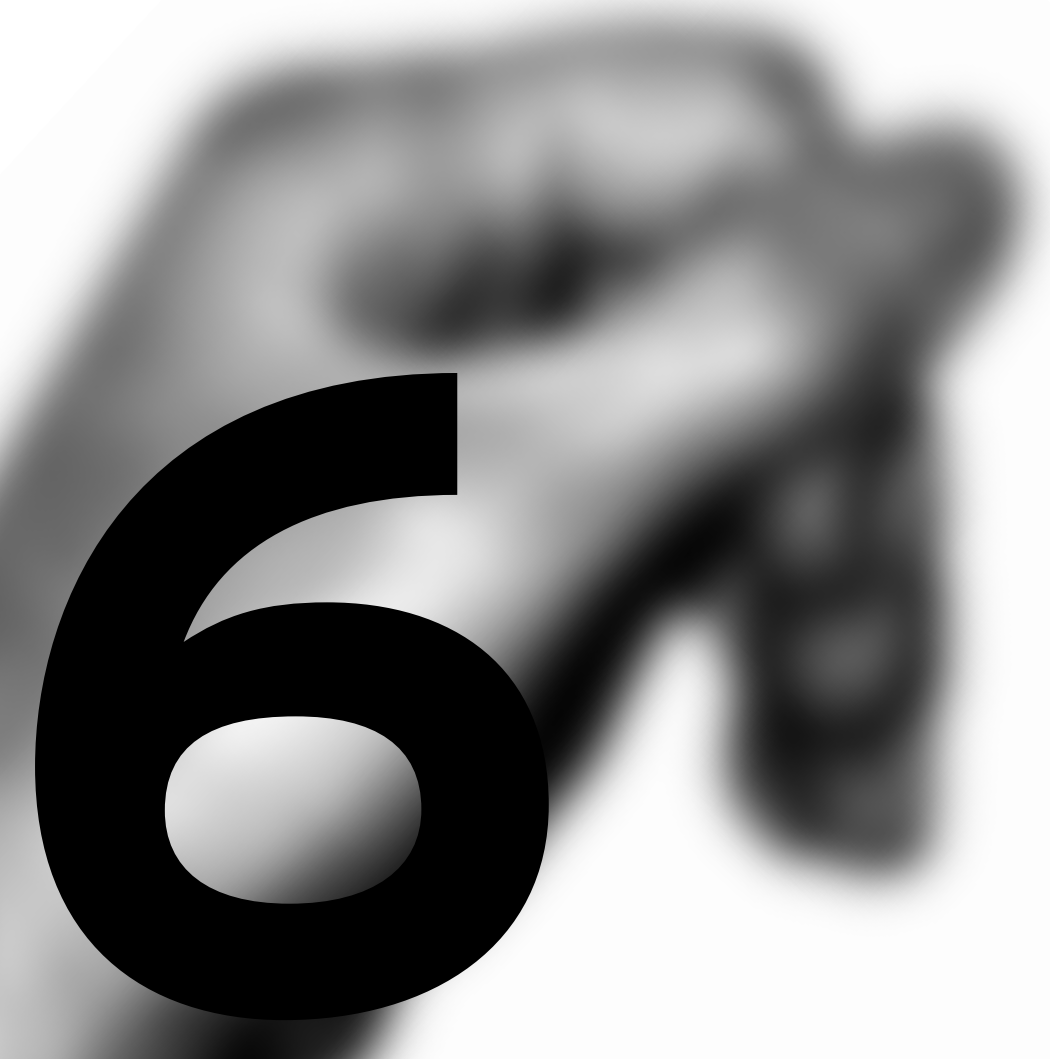




\section{Catastrophic thinking is}

\section{associated with finger stiffness}

\section{after distal radius fracture}

\section{surgery}

T. Teunis

A.G. Bot

E.R. Thornton

D. Ring

J Orthop Trauma. 2015 Oct;29(10):e414-20.

Presented at the European Congress of Trauma \& Emergency Surgery (2015),

Annual Meeting of the American Academy of Orthopaedic Surgeons (2015),

and the Annual Meeting of the Orthopaedic Trauma Association (2014). 
Importance Finger stiffness is common after distal radius fracture, but variations in pathophysiology such as trauma mechanism or fracture type do not seem to adequately account for the variability in stiffness.

Objectives To identify demographic, injury-related or psychological factors associated with finger stiffness at suture removal and 6 weeks after distal radius fracture surgery.

Design Prospective cohort study.

Setting Level I Academic Urban Trauma Center.

Participants 116 adult patients who underwent open reduction and internal fixation of their distal radius fracture, of whom 96 (83\%) were available 6 weeks after surgery. Mean age $55 \pm 14$ years (range 22-90), and 85 (73\%) were female.

Predictor variables At suture removal we recorded patients' demographics, AO fracture type, carpal tunnel release at the time of surgery, pain catastrophizing scale, Whiteley Index, and Patient Health Questionnaire-9. We also recorded pre-reduction and post-surgery radiographic fracture characteristics.

Main Outcomes Distance to palmar crease and active flexion of the thumb through small finger at suture removal and at 6 weeks.

Results Female sex, being married, specific surgeons, carpal tunnel release, AO type $C$ fractures, and greater catastrophic thinking were associated with increased distance to palmar crease at suture removal. At 6 weeks, greater catastrophic thinking was the only factor associated with increased distance to palmar crease.

Conclusion Catastrophic thinking was a consistent and major determinant of finger stiffness at suture removal and six weeks after injury. Future research should assess if treatments that ameliorate catastrophic thinking can facilitate recovery of finger motion after operative treatment of a distal radius fracture.

Level of Evidence Prognostic level I 


\section{INTRODUCTION}

Finger stiffness is common after fracture of the distal radius. Variations in pathophysiology such as trauma mechanism or fracture type do not seem to adequately account for the variability in finger stiffness. For example, low and medium energy trauma lead to substantial finger stiffness more than high-energy fractures in a prior study.'

The majority of studies on finger stiffness after distal radius fracture usually take the perspective that patients with substantial stiffness may have some as yet poorly understood underlying pathophysiological process variously termed "algodystrophy", "causalgia", "reflex sympathetic dystrophy", or "complex regional pain syndrome". Until there is objective, reproducible evidence of an underlying pathophysiological process, it is probably better to refer to this process descriptively ("disproportionate pain and disability") and address it on the continuum that it occurs rather than trying to categorize patients as diseased or not diseased. ${ }^{2,3}$ Knowledge of factors associated with stiff fingers after operative treatment of a fracture of the distal radius might improve recovery. This study tested the primary null hypothesis that there are no demographic, injuryrelated, or psychological factors associated with finger stiffness measured by distance to palmar crease at suture removal after volar plate fixation of a fracture of the distal radius. Secondary study questions addressed stiffness measured by different methods at suture removal and 6 weeks after surgery.

\section{PATIENTS \& METHODS}

Study design

After approval by our institutional review board, we prospectively enrolled 116 adult patients with a distal radius fracture treated with a volar locking plate between December 2009 and April 2014. Patients were enrolled at suture removal, on average 11 days (standard deviation/SD \pm 4.8 days) after surgery (20 \pm 7.6 days after injury) at our level 1 trauma center. We excluded patients with (previous) ipsilateral fractures except of the ulna, polytrauma, pathologic or open fractures, treated more than 4 weeks after trauma, unable to complete enrollment forms due to any mental status or language problems (e.g. dementia, head injury, overall illness), or with lack of near-normal finger motion of their uninjured hand. After informed consent we recorded patients' age, sex, body mass index, smoking, pain conditions, marital status, employment, years of education, AO fracture type (extra-articular, partial- or complete articular ${ }^{4}$ ), carpal tunnel release at the time of surgery, and whether the injury involved the dominant hand. We also measured the following radiographic parameters at the time of injury prior to reduction and after surgery: (1) ulnarward inclination; (2) ulnar variance; (3) volar tilt; (4) ulna intact. ${ }^{5}$ Patients completed the pain catastrophizing scale, Whiteley Index, Patient Health Questionnaire-9 and Disabilities of the Arm, Shoulder and Hand (DASH) questionnaire. Patients reported pain intensity on an 11-point ordinal scale. Finger stiffness was measured by distance to palmar crease and active flexion of the index through small finger; thumb range of motion was recorded separately. Approximately 6 weeks (mean $44 \pm 15$ days) after surgery we measured motion, DASH, and pain. 


\section{Study population}

We enrolled 116 patients, mean age $55 \pm 14$ years (range 22-90), of whom 73\% ( $n=85)$ were female. Ninety-six patients (83\%) were also evaluated 6 weeks after surgery. Eight patients did not attend the second evaluation because they sought additional care closer to home and 12 were missed by the research assistant when their appointment was cancelled and rescheduled (Table 1). Patients were treated by 11 surgeons of whom 3 treated $98(85 \%)$ of all patients, for analysis the remaining 9 were grouped as 'others'. The temporary post-operative plaster splint applied in the operating room ended at the distal palmar crease and did restrict finger motion. The specifics varied by surgeon, but in general patients returned within a few days of surgery and were given a removable custom thermoplastic splint made by a hand therapist for comfort and encouraged to wean out of it. Active, self-assisted stretching exercises of the fingers and forearm were started immediately and wrist stretches were added as comfort allowed. Patients were encouraged to keep their shoulder limber.

\section{Table 1. Baseline characteristics of patient at suture removal and after 6 weeks}

\section{Demographic variables}

Patients

Age (years, range)

Women

Body mass index

Tobacco use

Other pain condition

- Muskuloskeletal

- Migraines

- Unknown

Marital status

- Single

- Partner/married

- Separated/widowed

Employed at time of fracture

Education (years)

Treating Surgeon

- Surgeon 1

- Surgeon 2

- Surgeon 3

- Others

\section{Injury related variables}

Injury to dominant side

Fracture reduced

Carpal tunnel release at the time of surgery

A0 classification

-A

- B

- C

Ulna intact

Radiographic parameters after injury

- Ulnarward inclination

- Ulnar variance

- Volar tilt

\section{At suture removal}

$116(100 \%)$

$55 \pm 14(22-90)$

$85(73 \%)$

$26 \pm 5.7$

$6(5.2 \%)$

$19(16 \%)$

16

2

1

25 (22\%)

72 (62\%)

19 (17\%)

$61(53 \%)$

$16 \pm 2.7$

$19(16 \%)$

$66(57 \%)$

$13(11 \%)$

$18(16 \%)$

$51(44 \%)$

$106(91 \%)$

$23(20 \%)$

$52(45 \%)$

$10(8.6 \%)$

$54(47 \%)$

55 (47\%)

$11^{\circ} \pm 9.0^{\circ}$

$1.2 \pm 5.3 \mathrm{~mm}$

$-13^{\circ} \pm 21^{\circ}$

\section{Six weeks after surgery}

$96(83 \%)$

$57 \pm 14(22-90)$

$74(77 \%)$

$25 \pm 5.5$

$2(2.1 \%)$

$17(18 \%)$

14

2

1

$15(16 \%)$

$64(67 \%)$

$17(18 \%)$

$54(56 \%)$

$16 \pm 2.6$

$17(18 \%)$

$55(57 \%)$

$8(8.3 \%)$

$16(17 \%)$

$44(46 \%)$

87 (91\%)

$19(20 \%)$

45 (47\%)

$6(6.3 \%)$

45 (47\%)

45 (47\%)

$12^{\circ} 9.0^{\circ}$

$0.83 \pm 5.3 \mathrm{~mm}$

$-13^{\circ} \pm 22^{\circ}$ 
Radiographic parameters after surgery

- Ulnarward inclination

$19^{\circ} \pm 4.7^{\circ}$

$-0.32 \pm 2.7 \mathrm{~mm}$

$5.0^{\circ} \pm 9.0^{\circ}$

$17 \pm 5.9$

$21 \pm 6.0$

$4.0 \pm 4.1$

$3.6 \pm 2.9$

$45 \pm 20$

$5.1 \pm 6.8 \mathrm{~cm}$

$885^{\circ} \pm 156^{\circ}$

$86 \% \pm 14 \%$

$163^{\circ} \pm 50^{\circ}$

$70 \% \pm 22 \%$ $20^{\circ} \pm 4.7^{\circ}$

$-0.51 \pm 2.5 \mathrm{~mm}$

$5.1^{\circ} \pm 9.1^{\circ}$

$17 \pm 5.6$

$21 \pm 6.4$

$3.9 \pm 4.0$

$2.0 \pm 2.3$

$23 \pm 17$

$1.7 \pm 4.5 \mathrm{~cm}$

$988^{\circ} \pm 115^{\circ}$

$96 \% \pm 9.4 \%$

$212^{\circ} \pm 42^{\circ}$

$90 \% \pm 16 \%$

Continuous variables as mean \pm standard deviation; discrete data as number (percentage)

\section{Questionnaires}

The pain catastrophizing scale measures misinterpretation or overinterpretation of nociception (catastrophic thinking). It comprises 13 items, scored on a 4-point Likert scale, ranging from 1 (not at all) to 4 (all the time). The total score ranges from 13-52 points with a higher score indicating more pain catastrophizing. ${ }^{6}$ The Whiteley Index assesses heightened illness concern. This questionnaire contains 14 questions scored using a 5-point Likert scale between 1 (not at all) to 5 (a great deal). The total score ranges from 14 to 70 with a higher score indicating greater illness concern. ${ }^{7}$

Symptoms of depression were measured using the Patient Health Questionnaire-9, which contains 9 questions answered on a 4-point Likert scale, ranging from 0 (not at all) to 3 (nearly every day). Scores from 0 to 27 are possible, with higher scores indicating more depressive symptoms. ${ }^{8}$

Patients rated their pain intensity on an 11-point ordinal scale, ranging from 0 to 10 , where 0 was no pain and 10 the worst pain ever. ${ }^{9}$

The DASH questionnaire evaluates arm-specific disability. It consists of 30 questions scored on 5-point Likert scales, ranging from 1 (no problems/pain) to 5 (impossible). Scores range between 0 and 100 points, a higher score indicating worse upper extremity specific disability and pain. ${ }^{10}$

\section{Finger stiffness}

A research fellow not involved with the treatment of the patients performed all measurements. To establish the distance to palmar crease, we asked patients to make a fist and determined the distance from nail tip to its respective most distal palmar crease for each individual digit using a ruler. We defined total distance to palmar crease as the sum of the values for the index, long, ring, and small fingers. 
Active flexion was measured using a handheld goniometer. We calculated total active flexion of the index through small finger by summing flexion at the metacarpo-phalangeal, proximal interphalangeal and distal interphalangeal joints (normal motion: $1080^{\circ}$ ). Contrary to previous study ${ }^{\prime \prime}$, we found a high correlation between index through small finger range of motion and distance to palmar crease of $-0.74(P<0.001)$ both at suture removal and 6 weeks, indicating both to be valid measures of finger stiffness.

Total active range of motion at the thumb combined active flexion at the metacarpo-phalangeal and interphalangeal joint, as well as palmar and radial abduction (normal motion: $240^{\circ}$ ).

\section{Statistical Analysis}

To identify independent predictors of finger stiffness we created 6 multivariable models: (1) distance to palmar crease at suture removal; (2) distance to palmar crease at 6 weeks after surgery; (3) range of motion of the index through small finger at suture removal; (4) finger range of motion at 6 weeks after surgery; (5) thumb range of motion at suture removal; and (6) thumb range of motion at 6 weeks. Multiple linear regression models were created by entering all variables associated with each of the our six response variables on exploratory bivariate analysis with $P<0.10$. Adjusted $\mathrm{R}^{2}$ indicates how much variability in the outcome variable the model accounts for. The partial $R^{2}$ indicates for how much variability each variable accounts for by itself. Continuous variables are described as mean \pm SD, discrete variables as proportions. We compared continuous and discrete variables by unpaired Student t-test or analysis of variance, continous variables by Pearson correlation. Table 2 reports all significant explanatory variables, bivariate analyses are reported in Appendix 1A-C, full multivariable models are available in Appendix 2.

We used multiple linear imputation for missing values (number of imputations set to 40): 2 (1.7\%) education, 23 (20\%) volar tilt after fracture, 2 (1.7\%) volar tilt after surgery, 2 (1.7\%) ulnar variance after surgery, distance to palmar crease of $12(2.6 \%)$ fingers at suture removal and $8(1.7 \%)$ at 6 weeks after surgery, motion of 8 $(0.57 \%)$ finger and $4(0.86 \%)$ thumb joints at suture removal, and $1(0.22 \%)$ at 6 weeks after surgery. Multiple linear imputation maintains the overall variability in the data while preserving relationships with other variables. All $\mathrm{R}^{2 \text { 's }}$ are the average of the 40 imputed sets.

A priori power analysis indicated that a sample of 116 patients would provide $90 \%$ statistical power, with set at 0.05 , for an effect size $f^{2}=0.15$ for a regression with five predictors. This means that we would detect an explanatory variable as a significant factor if it accounted for $7 \%$ or more of the variability in stiffness, assuming our complete model would account for $25 \%$ of the variability.

\section{RESULTS}

Distance to palmar crease - suture removal

Accounting for potential interaction of variables using multivariable analysis, male sex ( $\beta$ regression coefficient [ $\beta]-2.7,95 \%$ confidence interval $[\mathrm{Cl}]-5.3$ to -0.073 , partial $\left.\mathrm{R}^{2} 0.023, P=0.044\right)$, being married $(\beta 4.7,95 \% \mathrm{Cl}$ 1.4 to 8.0 , partial $R^{2} 0.050, P=0.006$ ), being operated by any of the 9 other surgeons $(\beta 4.9,95 \% \mathrm{Cl} 1.2$ to 8.6 , partial $\left.\mathrm{R}^{2} 0.042, P=0.010\right)$, concomitant carpal tunnel release $\left(\beta 4.0,95 \% \mathrm{Cl} 1.0\right.$ to 7.0 , partial $\mathrm{R}^{2} 0.043, P=$ $0.0090)$, AO type $C$ fracture $\left(\beta 2.6,95 \% \mathrm{Cl} 0.033\right.$ to 5.1 , partial $\mathrm{R}^{2} 0.022, P=0.047$ ), and greater catastrophic thinking ( $\beta 0.32,95 \% \mathrm{Cl} 0.068$ to 0.57 , partial $\mathrm{R}^{2} 0.38, P=0.014$ ) were associated with greater distance to palmar crease at suture removal (adjusted $\mathrm{R}^{2} 0.41, P<0.001$; Table 2). 
Table 2. Multivariable analysis of finger stiffness at suture removal and 6 weeks after surgery

\section{Distance to palmar crease}

Suture removal

Male

Marital status: partner/married

Surgeon: others

Carpal tunnel release

AO fracture type $C$

Pain Catastrophizing Scale

6 weeks after fracture

Pain Catastrophizing Scale

\section{Finger range of motion}

Suture removal

Pain Catastrophizing Scale

6 weeks after fracture

Age (years)

Education (years)

Fracture reduced

Pain Catastrophizing Scale

\section{Thumb motion}

Suture removal

Male sex

Other pain condition

Surgeon 3

Surgeon: others

A0 fracture type $C$

Pain Catastrophizing Scale

6 weeks after fracture

Age (years)

Marital status: partner/married

Surgeon 2
Regression coefficient ( $95 \%$ confidence interval)

Standard error P value

$-2.7(-5.3$ to -0.073$)$

4.7 (1.4 to 8.0)

4.9 (1.2 to 8.6)

$4.0(1.0$ to 7.0$)$

$2.6(0.033$ to 5.1$)$

$0.32(0.068$ to 0.57$)$

$0.40(0.22$ to 0.59$)$

0.092

$<0.001$

$-4.7(-9.3$ to -0.069$)$

2.3

0.047

$-3.2(-4.7$ to -1.7$)$
$10(2.7-17)$
$75(7.1$ to 143$)$
$-5.9(-11$ to -1.3$)$

27 (7.6 to 46)

$-25(-48$ to -2.3$)$

$-39(-74$ to -3.7$)$

$-35(-65$ to -5.2$)$

$-22(-42$ to -2.7$)$

$-1.6(-3.1$ to -0.13$)$

$-0.78(-1.4$ to -0.12$)$
$-26(-51$ to -0.64$)$
$28(5.8$ to 49$)$

0.75

3.7

34

2.3

$<0.001$

0.008

0.031

0.012

$\begin{array}{ll}0.026 & 0.027\end{array}$

Partial R ${ }^{2}$

$0.14 \quad 0.38$

$0.14 \quad 0.38$

0.021

0.28

Adjusted R

0.41

0.043

0.022

0.038

0.023

.050

0.042

0.12

0.051

0.027

0.039

Only significant explanatory variables are reported, see Appendix 2 for the full multivariable models.

Missing values are imputed using multiple linear imputation (number of imputations set to 40$): 2$ (1.7\%) education, 23 (20\%) volar tilt after fracture, 2 (1.7\%) volar tilt after surgery, $2(1.7 \%)$ ulnar variance after surgery, distance to palmar crease of $12(2.6 \%)$ fingers at suture removal and $8(1.7 \%)$ at 6 weeks after surgery, motion of $8(0.57 \%)$ finger and $4(0.86 \%)$ thumb joints at suture removal, and $1(0.22 \%)$ at 6 weeks after surgery. Adjusted $\mathrm{R}^{2}$ is the average of the 40 imputed sets

\section{Distance to palmar crease - 6 weeks}

Greater catastrophic thinking $\left(\beta 0.40,95 \% \mathrm{Cl} 0.22\right.$ to 0.59 , partial $\left.\mathrm{R}^{2} 0.14, P<0.001\right)$ was the only factor independently associated with greater distance to palmar crease 6 weeks after surgery (adjusted $\mathrm{R}^{2} 0.38, P$ $<0.001$; Table 2). The $\beta$ regression coefficient indicates that every point increase in catastrophic thinking on average results in a $0.40 \mathrm{~cm}$ greater distance to palmar crease. 


\section{Range of motion - suture removal}

Also at suture removal greater catastrophic thinking $\left(\beta-4.7,95 \% \mathrm{Cl}-9.3\right.$ to -0.069 , partial $\left.\mathrm{R}^{2} 0.021, P<0.001\right)$ was the only factor independently associated with less finger range of motion at suture removal (adjusted $\mathrm{R}^{2}$ $0.28, P<0.001$; Table 2). Every point increase in catastrophic thinking on average results in a $4.7^{\circ}$ less finger motion.

\section{Range of motion - 6 weeks}

Older age $\left(\beta-3.2,95 \% \mathrm{Cl}-4.7\right.$ to -1.7 , partial $\left.\mathrm{R}^{2} 0.12, P<0.001\right)$, less education $(\beta 10,95 \% \mathrm{Cl} 2.7$ to 17 , partial $\mathrm{R}^{2}$ 0.051, $\left.P=0.008\right)$, unreduced fractures $\left(\beta 75,95 \% \mathrm{Cl} 7.1\right.$ to 143 , partial $\left.\mathrm{R}^{2} 0.027, P=0.031\right)$ and greater catastrophic thinking $\left(\beta-5.9,95 \% \mathrm{Cl}-11\right.$ to -1.3 , partial $\left.\mathrm{R}^{2} 0.039, P=0.012\right)$ were independently associated with less finger motion 6 weeks after surgery (adjusted $\mathrm{R}^{2} 0.41, P<0.001$; Table 2 ).

\section{Thumb range of motion - suture removal}

Female sex $\left(\beta 27,95 \% \mathrm{Cl} 7.6\right.$ to 46 , partial $\left.\mathrm{R}^{2} 0.048, P=0.007\right)$, having a pain condition $(\beta-25,95 \% \mathrm{Cl}-48$ to -2.3 , partial $\left.R^{2} 0.027, P=0.032\right)$, being treated by surgeon $3\left(\beta-39,95 \% \mathrm{Cl}-74\right.$ to -3.7 , partial $\mathrm{R}^{2} 0.031$, $P=0.028)$, or any of the other 9 surgeons $\left(\beta-35,95 \% \mathrm{Cl}-65\right.$ to -5.2 , partial $\left.\mathrm{R}^{2} 0.032, P=0.022\right)$, AO type $\mathrm{C}$ fracture $\left(\beta-22,95 \% \mathrm{Cl}-42\right.$ to -2.7 , partial $\left.\mathrm{R}^{2} 0.030, P=0.026\right)$, and greater catastrophic thinking $(\beta-1.6,95 \%$ $\mathrm{Cl}-3.1-0.13$, partial $\mathrm{R}^{2} 0.027, P=0.033$ ) were independently associated with less thumb motion at suture removal (adjusted $\mathrm{R}^{2} 0.23, P<0.001$; Table 2).

\section{Thumb range of motion - 6 weeks}

Independently associated with less thumb motion 6 weeks after surgery were older age $(\beta-0.78,95 \% \mathrm{Cl}-1.4$ to -0.12, partial $\left.\mathrm{R}^{2} 0.048, P=0.021\right)$, being married $\left(\beta-26,95 \% \mathrm{Cl}-0.51\right.$ to -0.64 , partial $\left.\mathrm{R}^{2} 0.033, P=0.045\right)$, or not being treated by surgeon $2\left(\beta 28,95 \% \mathrm{Cl} 5.8\right.$ to 49 , partial $\left.\mathrm{R}^{2} 0.056, P=0.014\right)$ (adjusted $\mathrm{R}^{2} 0.30, P<0.001$; Table 2).

\section{DISCUSSION}

Difference in trauma mechanism, fracture type, and injury severity do not account for much of the variability in finger stiffness after a distal radius fracture. Evidence that pain intensity and magnitude of disability are explained largely by psychosocial factors ${ }^{12}$ encouraged us to see if finger motion was also affected. Catastrophic thinking was a consistent and major determinant of finger stiffness at suture removal and six weeks after injury.

This study has some limitations. First, this study was powered for our primary study question, but had $85 \%$ power for the secondary study questions. Secondly, we didn't quantify the extent of articular incongruity. As about half of the fractures were AO type A, they would have no incongruity, resulting in a floor effect; this variable would not be able to differentiate reliably. Instead we included AO fracture type as a surrogate measure of joint incongruity.

A variety of labels (e.g., "algodystrophy", "causalgia", "complex regional pain syndrome") represent a seemingly unhelpful attempt to dichotomize "greater stiffness than expected" and make it difficult to compare our study to prior studies (Table 3). 
Table 3. Summary of previous studies on disproportionate pain and disability after distal radius fracture

\begin{tabular}{|c|c|c|c|c|c|}
\hline Author & Type & $\begin{array}{l}\text { Affected/Total } \\
\text { (n) }\end{array}$ & Treatment & FU (weeks) & Criteria \\
\hline Jellad et al., 2014 & $P$ & $29 / 90$ & NO & 36 & Veldman \\
\hline Dilek at al., 2010 & $P$ & $13 / 50$ & NO & 8 & IASP \\
\hline Puchalski \& Zyluk, 2005 & $P$ & 18507 & NO \& percutanous pinning & 8 & Zyluk \& Veldman \\
\hline Dijkstra et al.. 2003 & $P$ & 32143 & N0 \& operative* & 52 & IASP \\
\hline Field \& Gardner, 1997 & $P$ & $24 / 100$ & NO & 9 & Atkins \\
\hline Field et al., 1994 & $P$ & 42178 & NO & 9 & Atkins \\
\hline Bickerstaff et al., 1994 & $P$ & $77 / 274$ & NO & 52 & Atkins \\
\hline Atkins et al., 1990 & $P$ & $23 / 59$ & NO & 9 & Atkins \\
\hline Pollack et al., 1980 & $C$ & $40 / 60$ & NO & NA & Not reported \\
\hline Author & \multicolumn{2}{|c|}{ Demographics } & \multicolumn{2}{|l|}{ Measured variables } & Outcome Summary \\
\hline Jellad et al., 2014 & \multicolumn{2}{|c|}{$\begin{array}{l}\text { Age, sex, medical history, } \\
\text { profession, education, } \\
\text { socioeconomic status, and } \\
\text { dominant hand }\end{array}$} & $\begin{array}{l}\text { Trauma mechanism, radial } \\
\text { translation, volar inclination, } \\
\text { epiphyseal shortening, } \\
\text { radioulnar index, sagittal } \\
\text { inclination }\end{array}$ & $\begin{array}{l}\text { Hospital anxiety and } \\
\text { depression scale }\end{array}$ & $\begin{array}{l}\text { Female sex and low/medium } \\
\text { energy trauma were associated } \\
\text { with "complex regional pain } \\
\text { syndrome" }\end{array}$ \\
\hline Dilek at al., 2010 & \multicolumn{2}{|c|}{$\begin{array}{l}\text { Age, sex, education, } \\
\text { smoking, marital status, } \\
\text { and psychiatric and } \\
\text { systemic diseases }\end{array}$} & Not evaluated & $\begin{array}{l}\text { Anxiety Sensitivity Index, } \\
\text { Toronto Alexithymia Scale-20, } \\
\text { StateTrait Anxiety Inventory I } \\
\text { \& II, and the Beck Depression } \\
\text { Inventory }\end{array}$ & $\begin{array}{l}\text { Higher anxiety on State Trait } \\
\text { Anxiety Inventory II was } \\
\text { associated with "complex } \\
\text { regional pain syndrome" }\end{array}$ \\
\hline Puchalski \& Zyluk, 2005 & \multicolumn{2}{|c|}{ Not evaluated } & Not evaluated & $\begin{array}{l}\text { Eysenck Personality } \\
\text { Questionnaire, Adjectives } \\
\text { Checklist (personality } \\
\text { traits), Beck Depression } \\
\text { Inventory/Yesavage's Geriatric } \\
\text { Depression Scale }\end{array}$ & $\begin{array}{l}\text { No predictors of "complex } \\
\text { regional pain syndrome" }\end{array}$ \\
\hline Dijkstra et al.. 2003 & \multicolumn{2}{|c|}{$\begin{array}{l}\text { Age, sex, life events, } \\
\text { (non)dominant side, and } \\
\text { psychiatric history }\end{array}$} & Number of repositions & $\begin{array}{l}\text { Social Readjustment } \\
\text { Rating Scale and Symptom } \\
\text { Checklist-90 }\end{array}$ & $\begin{array}{l}\text { No predictors of "complex } \\
\text { regional pain syndrome" }\end{array}$ \\
\hline Field \& Gardner, 1997 & \multicolumn{2}{|c|}{ Not evaluated } & Not evaluated & $\begin{array}{l}\text { General Health } \\
\text { Questionnaire-30 }\end{array}$ & No predictors of "algpdystrophy" \\
\hline Field et al., 1994 & \multicolumn{2}{|c|}{ Not evaluated } & Cast tightness & Not evaluated & $\begin{array}{l}\text { Tighter cast at 1,2 and } 3 \\
\text { weeks was associated with } \\
\text { "algodystrophy" }\end{array}$ \\
\hline Bickerstaff et al., 1994 & \multicolumn{2}{|c|}{$\begin{array}{l}\text { Age, sex, (non)dominant } \\
\text { side, and time in cast }\end{array}$} & $\begin{array}{l}\text { Frykman classification and } \\
\text { number of reductions }\end{array}$ & Not evaluated & $\begin{array}{l}\text { "Algodystrophy" was more } \\
\text { common in Frykman type VIII } \\
\text { fractures and less common in } \\
\text { type I fractures. It was also more } \\
\text { common in reduced fractures } \\
\text { than unreduced fractures }\end{array}$ \\
\hline Atkins et al., 1990 & \multicolumn{2}{|c|}{$\begin{array}{l}\text { Age, sex, (non)dominant } \\
\text { side, and time in cast }\end{array}$} & $\begin{array}{l}\text { Frykman classification, number } \\
\text { of reductions, quality of the } \\
\text { reduction, final position, and } \\
\text { median nerve compression }\end{array}$ & Not evaluated & No predictors of "algpdystrophy" \\
\hline Pollack et al., 1980 & \multicolumn{2}{|c|}{ Not evaluated } & Not evaluated & $\begin{array}{l}\text { Freiburger } \\
\text { Persönlichkeitsinventar } \\
\text { [personality test] and } \\
\text { Ängstlichkeitsfragebogen } \\
\text { [anxiety questionnaire] }\end{array}$ & $\begin{array}{l}\text { Identified Type A and B"Sudeck } \\
\text { personality" }\end{array}$ \\
\hline
\end{tabular}


We found 5 studies that previously assessed demographic variables as predictors for "complex regional pain syndrome" or "algodystrophy" after distal radius fracture.1,13-16 One study found women more commonly affected.' In our study women had a higher distance to palmar crease and less thumb motion at suture, but not at 6 weeks after surgery. Two studies found no difference in level of education (measured in 3 categories: elementary school, high school, university). ${ }^{1,13}$ Education evaluated on a continuous scale was associated with decreased range of motion 6 weeks after surgery in our study. Level of education and job satisfaction are associated with other chronic pain conditions. ${ }^{17}$

Injury related variables were assessed in 5 studies. ${ }^{1,14-16,18}$ Low and medium energy trauma' and cast tightness ${ }^{18}$ - but not cast changes - have been associated with "complex regional pain syndrome" or "algodystrophy".

Cast tightness is somewhat subjective from both the patient and surgeon point of view. One study found that "algodystrophy" was more common in reduced fractures as compared to unreduced fracture. The authors suggest this is related to the higher extent of displacement (requiring reduction), but don't measure displacement directly. We found that patients with unreduced fractures had less finger motion 6 weeks after trauma. Also, the extent of displacement before or after surgery was not related to finger motion in our study. "Algodytrophy" was more frequent in Frykman fracture type VIII (intra-articular fracture involving the radiocarpal and radioulnar joint with an ulnar fracture ${ }^{19}$ ), and less common in Frykman type I fractures (extraarticular distal radius fracture $\left.{ }^{19}\right) .{ }^{16}$ We found $A O$ type $C$ fractures and carpal tunnel release at the time of surgery to be associated with increased distance to palmar crease only at suture removal, but not at 6 weeks. Another study found that in cadavers decreased thumb interphalangeal joint flexion might be related to partial stripping of the flexor pollicis longus muscle from investing fascia and bone. ${ }^{20}$ It's possible that differences in surgical technique might explain why patients differed in thumb motion between surgeons at suture removal and 6 weeks after fracture, although all surgeons sweep the muscle ulnarward in a similar fashion so it might also be the type of plate or the type of retraction used.

Five studies measured psychological variables such as anxiety, ${ }^{1,13}$ depression, 1,13,21 personality type, 14,21,22 life events, ${ }^{14}$ and general psychological disturbance. ${ }^{23}$ One study identified 2 distinct personality types more common in "Sudeck disease", but did not find a statistically significant difference compared to their control group. ${ }^{22}$ Another study found a correlation between anxiety and "complex regional pain syndrome" on 1 of 4 scales used to measure anxiety. ${ }^{13}$

Catastrophic thinking (negative beliefs about pain leading to an overprotective response) was the factor most consistently associated with finger stiffness in our study. Unchecked, catastrophic thinking leads to fear and avoidance of activity that in turn causes stiffness and skin changes (swelling, shiny skin, changed in hair patterns) associated with disuse. ${ }^{24}$ Catastrophic thinking is also associated with developing chronic lower back pain ${ }^{25,26}$ and is associated with greater pain intensity and magnitude of disability after various soft-tissue injuries. ${ }^{27}$ A reduction in catastrophic thinking resulted in fewer symptoms and less disability in patients with low back pain ${ }^{25,26}$ - something future studies should explore after distal radius fracture. 


\section{REFERENCES}

1. Jellad A, Salah S, Ben Salah Frih Z. Complex regional pain syndrome type I: incidence and risk factors in patients with fracture of the distal radius. Arch Phys Med Rehabil. 2014;95(3):487-492.

2. Bot AG, Ring DC. Recovery after fracture of the distal radius. Hand Clin. 2012;28(2):235-243.

3. Ring D, Barth R, Barsky A. Evidence-based medicine: disproportionate pain and disability. J Hand Surg Am. 2010;35(8):1345-1347.

4. Marsh JL, Slongo TF, Agel J, et al. Fracture and dislocation classification compendium - 2007: Orthopaedic Trauma Association classification, database and outcomes committee. J Orthop Trauma. 2007;21(10 Suppl):S1-133.

5. Medoff RJ. Essential radiographic evaluation for distal radius fractures. Hand Clin. 2005;21 (3):279-288.

6. Sullivan MJL, Bishop SR, Pivik J. The Pain Catastrophizing Scale: Development and validation. Psychological Assessment. 1995;7(4):524-532.

7. Pilowsky I. Dimensions of hypochondriasis. Br J Psychiatry. 1967;113(494):89-93.

8. Kroenke K, Spitzer RL, Williams JB. The PHQ-9: validity of a brief depression severity measure. J Gen Intern Med. 2001;16(9):606-613.

9. Bijur PE, Silver W, Gallagher EJ. Reliability of the visual analog scale for measurement of acute pain. Acad Emerg Med. 2001;8(12):1153-1157.

10. Hudak PL, Amadio PC, Bombardier C. Development of an upper extremity outcome measure: the DASH (disabilities of the arm, shoulder and hand) [corrected]. The Upper Extremity Collaborative Group (UECG). Am J Ind Med. 1996;29(6):602608.

11. Macdermid JC, Fox E, Richards RS, Roth JH. Validity of pulp-to-palm distance as a measure of finger flexion. J Hand Surg Br. 2001;26(5):432-435.

12. MacDermid JC, Donner A, Richards RS, Roth JH. Patient versus injury factors as predictors of pain and disability six months after a distal radius fracture. J Clin Epidemiol. 2002;55(9):849-854.

13. Dilek B, Yemez B, Kizil R, et al. Anxious personality is a risk factor for developing complex regional pain syndrome type I. Rheumatol Int. 2012;32(4):915-920.

14. Dijkstra PU, Groothoff JW, ten Duis HJ, Geertzen JH. Incidence of complex regional pain syndrome type I after fractures of the distal radius. Eur J Pain. 2003;7(5):457-462.

15. Atkins RM, Duckworth T, Kanis JA. Features of algodystrophy after Colles' fracture. J Bone Joint Surg Br. 1990;72(1):105110.

16. Bickerstaff DR, Kanis JA. Algodystrophy: an under-recognized complication of minor trauma. Br J Rheumatol. 1994;33(3):240-248.

17. Blyth FM, March LM, Brnabic AJ, Jorm LR, Williamson M, Cousins MJ. Chronic pain in Australia: a prevalence study. Pain. 2001;89(2-3):127-134.

18. Field J, Protheroe DL, Atkins RM. Algodystrophy after Colles fractures is associated with secondary tightness of casts. J Bone Joint Surg Br. 1994;76(6):901-905.

19. Frykman G. Fracture of the distal radius including sequelae--shoulder-hand-finger syndrome, disturbance in the distal radio-ulnar joint and impairment of nerve function. A clinical and experimental study. Acta Orthop Scand. 1967;108(Suppl):1-153.

20. Chilelli BJ, Patel RM, Kalainov DM, Peng J, Zhang LQ. Flexor pollicis longus dysfunction after volar plate fixation of distal radius fractures. J Hand Surg Am. 2013;38(9):1691-1697.

21. Puchalski P, Zyluk A. Complex regional pain syndrome type 1 after fractures of the distal radius: a prospective study of the role of psychological factors. J Hand Surg Br. 2005;30(6):574-580.

22. Pollack HJ, Neumann R, Pollack E. [Sudeck's disease and the psyche]. Beitr Orthop Traumatol. 1980;27(8):463-468.

23. Field J, Gardner FV. Psychological distress associated with algodystrophy. J Hand Surg Br. 1997;22(1):100-101.

24. Linton SJ, Shaw WS. Impact of psychological factors in the experience of pain. Phys Ther. 2011;91(5):700-711.

25. Wertli MM, Burgstaller JM, Weiser S, Steurer J, Kofmehl R, Held U. Influence of catastrophizing on treatment outcome in patients with nonspecific low back pain: a systematic review. Spine (Phila Pa 1976). 2014;39(3):263-273.

26. Wertli MM, Eugster R, Held U, Steurer J, Kofmehl R, Weiser S. Catastrophizing-a prognostic factor for outcome in patients with low back pain: a systematic review. Spine J. 2014;14(11):2639-2657.

27. Sullivan MJ, Stanish W, Waite H, Sullivan M, Tripp DA. Catastrophizing, pain, and disability in patients with soft-tissue injuries. Pain. 1998;77(3):253-260. 
Appendix 1A. Bivariate analysis of factors associated with distance to palmar crease

Independent variables

\section{Demographics}

Age (years, r)

Sex

- Male

- Female

Body mass index ( $r)$

Tobacco use

- Yes

- No

Other pain condition

- Yes

- No

Marital status

- Single

- Partner/married

- Separated/widowed

Employed at time of fracture

- Yes

- No

Education (years, r)

Treating Surgeon

- Surgeon 1

- Surgeon 2

- Surgeon 3

-0thers

Injury related variables

Injury to dominant side

- Yes

- No

Fracture reduced

- Yes

- No

Carpal tunnel release during plating

- Yes

- No

A0 classification

-A

$\cdot B$

$\cdot C$

Ulna intact

$\begin{array}{ll}\text { - Yes } & 5.1 \pm 6.7 \\ \text { • No } & 5.0 \pm 6.8\end{array}$

Radiographic parameters after injury ( $r$ )

- Ulnarward inclination

- Ulnar variance

- Volar tilt
$5.1 \pm 6.2$

$-0.13$

0.0092

Enrollment

0.10

$3.2 \pm 4.5$

$5.7 \pm 7.3$

$-0.076$

$4.2 \pm 4.6$

$5.1 \pm 6.9$

$4.4 \pm 6.8$

$5.2 \pm 6.8$

$2.3 \pm 3.8$

$6.5 \pm 7.6$

$3.4 \pm 4.9$

$4.8 \pm 5.3$

$5.4 \pm 8.1$

$-0.10$

$6.6 \pm 7.8$

$2.4 \pm 3.5$

$7.9 \pm 8.5$

$11 \pm 8.2$

$5.0 \pm 7.2$

$4.8 \pm 6.5$

$7.5 \pm 9.2$

$9.6 \pm 7.8$

$3.9 \pm 6.0$

$3.4 \pm 5.1$

$4.0 \pm 4.6$

$6.9 \pm 8.0$

$5.0 \pm 6.8$

$-0.14$
DTPC digit II-IV (cm)

Pvalue

Follow-up

$P$ value

0.0051

0.44

$1.9 \pm 4.9$

0.040

0.70

$2.1 \pm 3.0$

0.90

$1.7 \pm 4.6$

0.64

$3.8 \pm 7.4$

0.037

$1.3 \pm 3.5$

0.013

$1.9 \pm 4.9$

$2.8 \pm 4.8$

$0.76 \pm 2.1$

0.017

$3.0 \pm 6.2$

$-0.28$

0.0069

$3.4 \pm 5.7$

0.035

$0.84 \pm 2.5$

$0.19 \pm 0.37$

$3.8 \pm 7.8$

$2.1 \pm 5.1$

$1.4 \pm 3.9$

$1.5 \pm 3.8$

0.21

$3.5 \pm 9.1$

$1.8 \pm 3.5$

$1.7 \pm 4.8$

$1.8 \pm 5.3$

0.70

$0.21 \pm 0.51$

$1.8 \pm 4.0$

0.97

$1.9 \pm 4.5$

0.67

$1.5 \pm 4.6$

$-0.14$

0.22

0.048

0.68

0.20

$-0.10$

0.38 
Radiographic parameters after surgery $(r)$

- Ulnarward inclination
- Ulnar variance
- Volar tilt

$-0.062$

$-0.11$

$-0.21$

0.24

Whiteley Index

0.15

0.16
0.0086

0.11

0.52

0.23

0.028

0.089
Patient Health Questionnaire
$-0.039$

0.71

$-0.19$

0.068

0.14

DTPC = distance to palmar crease; continuous variables as mean \pm standard deviation; Pearson correlation indicated by $r$;

$P$ value $<0.05$ indicates statistically significant finding.

\section{Appendix 1B. Bivariate analysis of factors associated with finger range of motion}

\section{Independent variables}

Demographics

Age (years, $r$ )

Sex

$$
\text { - Male }
$$

- Female

Body mass index ( $r$ )

Tobacco use

$$
\text { - Yes }
$$

- No

Other pain condition

- Yes

- No

Marital status

- Single

- Partner/married

- Separated/widowed

Employed at time of fracture

- Yes

- No

Education (years, $r$ )

Treating Surgeon

- Surgeon 1

- Surgeon 2

- Surgeon 3

- Others

\section{Injury related variables}

Injury to dominant side

$$
\text { - Yes }
$$$$
\text { - No }
$$

$881^{\circ} \pm 163^{\circ}$

$889^{\circ} \pm 147^{\circ}$

$889^{\circ} \pm 153^{\circ}$

racture reduced

- Yes

- No

$828^{\circ} \pm 187^{\circ}$

Carpal tunnel release during plating

- Yes

- No

$828^{\circ} \pm 157^{\circ}$

$899^{\circ} \pm 153^{\circ}$

A0 classification
-A
- B
- $C$
$918^{\circ} \pm 142^{\circ}$
$963^{\circ} \pm 72^{\circ}$
$839^{\circ} \pm 167^{\circ}$

\section{ROM digit II-IV}

$P$ value

Follow-up

Pvalue

0.012

$-0.45$

$<0.001$

0.18

0.62

$978^{\circ} \pm 89^{\circ}$

$992^{\circ} \pm 123^{\circ}$

0.63

$-0.19$

0.070

$948^{\circ} \pm 53^{\circ}$

$989^{\circ} \pm 116^{\circ}$

0.61

$938^{\circ} \pm 168^{\circ}$

$999^{\circ} \pm 99^{\circ}$

0.045

$1030^{\circ} \pm 97^{\circ}$

$986^{\circ} \pm 119^{\circ}$

$962^{\circ} \pm 113^{\circ}$

0.24

$1009^{\circ} \pm 84^{\circ}$

$963^{\circ} \pm 143^{\circ}$

0.32

0.0018

$970^{\circ} \pm 127^{\circ}$

$1013^{\circ} \pm 93^{\circ}$

$968^{\circ} \pm 92^{\circ}$

0.078

$934^{\circ} \pm 163^{\circ}$

$995^{\circ} \pm 127^{\circ}$

$983^{\circ} \pm 106^{\circ}$

0.59

$998^{\circ} \pm 104^{\circ}$

$897^{\circ} \pm 176^{\circ}$

$979^{\circ} \pm 81^{\circ}$

$991^{\circ} \pm 123^{\circ}$

$984^{\circ} \pm 136^{\circ}$

$1041^{\circ} \pm 53^{\circ}$

$986^{\circ} \pm 98^{\circ}$

0.52 
Ulna intact

- Yes

- No

Radiographic parameters after injury ( $r$ )

- Ulnarward inclination

- Ulnar variance

- Volar tilt

Radiographic parameters after surgery ( $r$ )

- Ulnarward inclination

- Ulnar variance

- Volar tilt

Psychological variables ( $r$ )

Pain Catastrophizing Scale

Whiteley Index

Patient Health Questionnaire $883^{\circ} \pm 158^{\circ}$

$886^{\circ} \pm 155^{\circ}$

0.15

0.052

0.044

0.067

0.11

0.084

-0.24
-0.083
-0.086
0.90

$989^{\circ} \pm 121^{\circ}$

$988^{\circ} \pm 112^{\circ}$

0.13

$-0.0098$

0.10

0.027

0.099

0.022

$-0.42$

$-0.22$

$-0.075$
0.97

0.27

0.93

0.37

0.80

0.34

0.83

$<0.001$

0.033

0.47

$\mathrm{ROM}=$ range of motion; continuous variables as mean \pm standard deviation; Pearson correlation indicated by $\mathrm{r}$; $\mathrm{P}$ value $<0.05$ indicates statistically significant finding.

\section{Appendix 1C. Bivariate analysis of factors associated with thumb range of motion}

\section{Independent variables}

\section{Demographics}

Age (years, r)

Sex

$$
\text { Male }
$$

Female

Body mass index ( $r$ )

Tobacco use
Yes
No

Other pain condition

Yes

No

Marital status

Single

Partner/married

Separated/widowed

Employed at time of fracture

Yes

No

Education (years, r)

Treating Surgeon

$\begin{array}{ll}\text { Surgeon 1 } & 155^{\circ} \pm 64^{\circ} \\ \text { Surgeon 2 } & 177^{\circ} \pm 46^{\circ} \\ \text { Surgeon 3 } & 150^{\circ} \pm 44^{\circ} \\ \text { Others } & 131^{\circ} \pm 31^{\circ}\end{array}$

Enrollment
-0.074
$182^{\circ} \pm 55^{\circ}$
$157^{\circ} \pm 46^{\circ}$
0.12
$157^{\circ} \pm 67^{\circ}$
$164^{\circ} \pm 49^{\circ}$

$146^{\circ} \pm 60^{\circ}$

$168^{\circ} \pm 48^{\circ}$

$178^{\circ} \pm 48^{\circ}$

$165^{\circ} \pm 44^{\circ}$

$168^{\circ} \pm 43^{\circ}$

$159^{\circ} \pm 57^{\circ}$

0.055

\section{Thumb ROM}

0.076

0.013

0.19

Follow-up

Pvalue

$-0.43$

$<0.001$

$217^{\circ} \pm 44^{\circ}$

$210^{\circ} \pm 42^{\circ}$

0.56

0.030

0.77

$218^{\circ} \pm 3.5^{\circ}$

$212^{\circ} \pm 43^{\circ}$

0.86

$187^{\circ} \pm 54^{\circ}$

$218^{\circ} \pm 37^{\circ}$

0.0066

0.26

$239^{\circ} \pm 45^{\circ}$

$207^{\circ} \pm 38^{\circ}$

0.025

$207^{\circ} \pm 48^{\circ}$

0.31

$223^{\circ} \pm 36^{\circ}$

$199^{\circ} \pm 46^{\circ}$

0.57

0.15

0.0059

0.002

$196^{\circ} \pm 52^{\circ}$

$222^{\circ} \pm 36^{\circ}$

$221^{\circ} \pm 23^{\circ}$

$190^{\circ} \pm 48^{\circ}$

Injury related variables

Injury to dominant side

$\begin{array}{llll}\text { Yes } & 159^{\circ} \pm 47^{\circ} & 208^{\circ} \pm 46^{\circ} \\ \text { No } & 168^{\circ} \pm 52^{\circ} & 0.32 & 216^{\circ} \pm 38^{\circ}\end{array}$


Fracture reduced

Yes $\quad 164^{\circ} \pm 50^{\circ}$

$214^{\circ} \pm 41^{\circ}$

No $158^{\circ} \pm 50^{\circ}$

Carpal tunnel release during plating
Yes

No

$142^{\circ} \pm 47^{\circ}$

$169^{\circ} \pm 50^{\circ}$

0.020

$174^{\circ} \pm 48^{\circ}$

$177^{\circ} \pm 35^{\circ}$

$152^{\circ} \pm 53^{\circ}$

0.061

B

C

Ulna intact

Yes

No

$161^{\circ} \pm 45^{\circ}$

$167^{\circ} \pm 56^{\circ}$

0.48

Radiographic parameters after injury (r)

Ulnarward inclination

0.087

Ulnar variance

0.0062

0.15

0.41

0.95

0.15

Radiographic parameters after surgery ( $r$ )

Ulnarward inclination

0.072

Ulnar variance

0.12

0.16

0.45

0.20

0.10

$-0.22$

0.0021

$-0.049$

Psychological variables ( $r$ )

Pain Catastrophizing Scale

Whiteley Index

0.020

0.98

0.60 $199^{\circ} \pm 55^{\circ}$

$210^{\circ} \pm 31^{\circ}$

$213^{\circ} \pm 44^{\circ}$

$212^{\circ} \pm 43^{\circ}$

$221^{\circ} \pm 25^{\circ}$

0.87

$211^{\circ} \pm 44^{\circ}$

$211^{\circ} \pm 46^{\circ}$

$213^{\circ} \pm 39^{\circ}$

0.04

0.73

$-0.028$

0.81

0.25

0.030

$-0.33$

0.0011

Patient Health Questionnaire

$-0.12$

0.25

$-0.0056$

0.96

$\mathrm{ROM}=$ range of motion; continuous variables as mean \pm standard deviation; Pearson correlation indicated by $r$; $P$ value $<0.05$ indicates statistically significant finding. 
Appendix 2. Full multivariable models of finger stiffness at suture removal and 6 weeks after surgery

\section{Distance to palmar crease}

Suture removal

Male

Marital status

Single

Partner/married

Separated/widowed

Surgeon

Surgeon 1

Surgeon 2

Surgeon 3

Others

Carpal tunnel release

A0 fracture classification

Type A

Type B

Type C

Volar tilt after surgery $\left(^{\circ}\right)$

Pain Catastrophizing Scale

Patient Health Questionnaire

6 weeks after fracture

Age (years)

Other pain condition

Employed

Education (years)

Surgeon

Surgeon 1

Surgeon 2

Surgeon 3

Others

Ulnar variance after surgery ( $\mathrm{mm}$ )

Pain Catastrophizing Scale

Whiteley Index

\section{Finger range of motion}

Suture removal

Age (years)

Employed

Education

Surgeon

Surgeon 1

Surgeon 2

Surgeon 3

Others

Carpal tunnel release

A0 fracture classification

Type A

Type B

Type C

Pain Catastrophizing Scale
Regression coefficient (95\% confidence interval)

Standard error P value

Partial R

Adjusted R

$-2.7(-5.3$ to -0.073$)$

1.3

0.044

0.023

reference value

4.7 (1.4 to 8.0)

$0.57(-3.4$ to 4.5$)$

1.7

0.006

0.050

2.0

0.78

reference value

-0.43 ( -4.0 to 3.1$)$

1.8

0.81

$2.5(-3.0$ to 8.0$)$

0.37

4.9 (1.2 to 8.6)

0.010

0.042

4.0 (1.0 to 7.0$)$

1.4

0.009

0.043

reference value

0.021 (-4.6 to 4.7)

$2.6(0.033$ to 5.1$)$

$-0.019(-0.15$ to 0.12$)$

1.1

2.8

0.99

0.32 (0.068 to 0.57 )

0.063

0.047

0.022

$0.17(-0.16$ to 0.50$)$

0.12

0.78

0.014

0.038

0.17

0.32

$0.045(-0.015$ to 0.11$)$

0.030

1.1

$1.0(-1.1$ to 3.2$)$

$-0.62(-2.3$ to 1.0$)$

$-0.25(-0.55$ to 0.051$)$

0.83

0.15

0.14

0.34

0.46

0.10

reference value

$-1.3(-3.4$ to 0.73$)$

1.0

0.20

$-0.60(-4.0$ to 2.8$)$

1.7

0.73

0.17 (-2.4 to 2.8)

0.89

$-0.26(-0.58$ to 0.055$)$

0.10

0.40 (0.22 to 0.59$)$

0.16

$<0.001$

0.14

$-0.045(-0.21$ to 0.11$)$

0.092

0.080

0.57

0.38

$-1.7(-3.6$ to 0.12$)$

0.94

0.067

38 (-16 to 93)

27

0.17

$9.0(-1.3$ to 19)

5.2

0.085

reference value

41 (-41 to 122)

$-69(-174$ to 35$)$

-77 (-168 to 14)

-45 (-115 to 24)

reference value

68 (-30 to 167)

50

0.17

$-46(-105$ to 14$)$

30

0.13

$-4.7(-9.3$ to -0.069$)$
0.33

0.19

0.096

0.20
0.41

. 


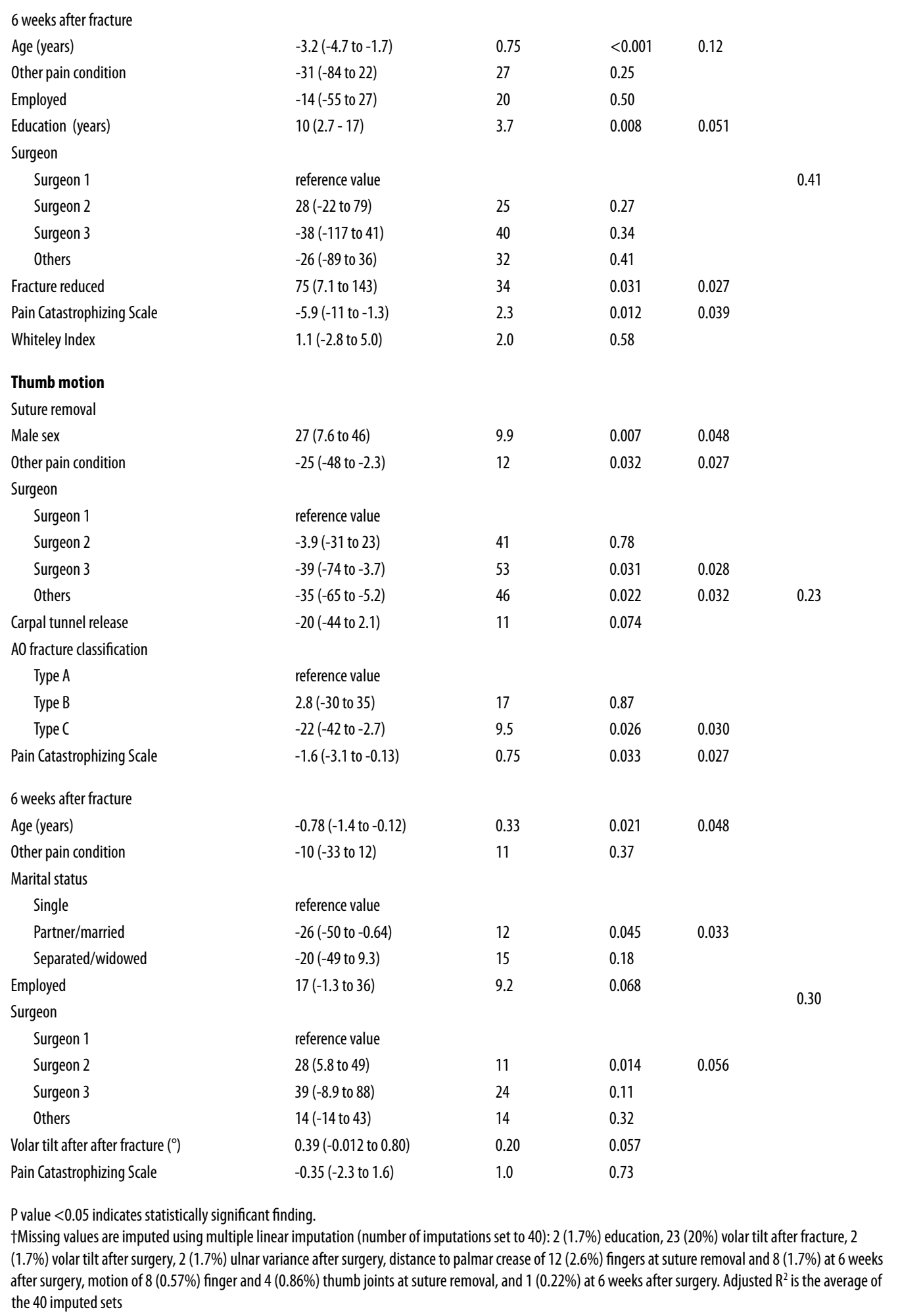


CHAPTER 


\title{
Changes in depression, health
}

anxiety and pain catastrophizing

\section{between enrollment and 1 month}

\section{after a radius fracture}

\author{
S. Golkari \\ T. Teunis \\ D. Ring \\ A.M. Vranceanu
}

Psychosomatics. 2015 Nov-Dec;56(6):652-7. 
Importance Psychological factors such as symptoms of depression, health anxiety, and catastrophic thinking can be quantified using validated questionnaires that have good test-retest reliability in stable individuals. However, the performance of these questionnaires during recovery is unknown.

Objectives To test the difference in symptoms of (1) depression, (2) health anxiety, and (3) catastrophic thinking between one and six weeks after injury to the radius.

Design Prospective cohort study.

Setting Level I Academic Urban Trauma Center.

Participants 69 adult patients with a minimally displaced radial head or distal radius fracture, of whom 55 (80\%) were available after 1 month. Mean age was $47 \pm 16$ years (range 20-80 years), and 39 were female.

Predictor variables patients' demographics, 11 -point ordinal numerical pain score and agreement with "no pain, no gain," Disability of the Arms, Shoulder, and Hand (DASH) questionnaire.

Main Outcomes Difference in Center for Epidemiologic Studies Depression (CESD) Scale, the Whiteley Index, and the Pain Catastrophizing Scale (PCS) between one and six weeks after injury.

Results CESD scores decreased by an average of $5 \pm 9$ points $(P<0.001)$ and Pain Catastrophizing Scale scores decreased by $2 \pm 6$ points $(P=0.0041)$. In multivariable analysis, decrease in CESD was associated with not having an additional pain condition, more days elapsed between injury and final evaluation, and stronger agreement with "no pain, no gain" (adjusted $\mathrm{R}^{2} 0.26, P=0.0006$ ). An increase in Whiteley scores was associated with fewer years of education $(R=-0.34 ; P=0.012)$. Changes in PCS scores were associated with marital status (single $-1.7 \pm 4.3$ vs. married $-4.6 \pm 6.0$ vs. separated $0.55 \pm 6.2, P=0.040$ ).

Conclusion Symptoms of depression and catastrophic thinking, but not health anxiety, improved during recovery after injury. If psychological measures are used as a screening tool to predict outcome after treatment, one should account for a patient's disease phase.

Level of Evidence Prognostic level I 


\section{INTRODUCTION}

Psychological factors (such as symptoms of depression, health anxiety, and catastrophic thinking) are associated with increased magnitude of disability and pain intensity in patients with musculoskeletal illness.' Those constructs can be quantified using validated questionnaires that have good test-retest reliability in stable individuals. ${ }^{2-4}$ However, the performance of these questionnaires during recovery is unknown. Symptoms of depression might be in part reactive and may change over time as patients recover. For example, a previous study showed symptoms of depression decreased from $51 \%$ to $20 \% 1$ year after hip fracture surgery. ${ }^{5}$ But health anxiety and catastrophic thinking may be more stable constructs that less affected by recovery. This study aimed to assess magnitude of change from baseline to 1 month for 3 important constructs associated with pain and disability in orthopedic patients. Specifically, this study tested the primary nullhypothesis that there is no difference in symptoms of (1) depression, (2) health anxiety, and (3) catastrophic thinking between approximately 1 and 6 weeks after injury among patients with non-operatively treated radial head and distal radius fractures. Secondarily, we assessed the association of demographic variables with change in questionnaire scores for depression, health anxiety, and pain catastrophizing.

\section{MATERIALS \& METHODS}

Study design \& patient population

Following institutional review board approval, we prospectively enrolled 69 adult patients with a minimally displaced radial head or distal radius fracture between December 2009 and May 2014. Inclusion criteria were a stable, isolated, non-operatively-treated fracture of the radial head or distal radius occurring no later than 14 days before enrollment. We excluded patients cognitively and physically unable to perform the required exercises, pregnant women, and patients unable to provide informed consent.

After the senior investigator diagnosed the isolated radial head or distal radius fracture, informed consent was obtained and the visit was paused to record: demographic variables (sex, age, smoking status, pre-existing pain conditions, marital status, employment status, years of education, and the type of immobilization), 11 -point ordinal numerical pain score, and agreement with "no pain, no gain," Disability of the Arms, Shoulder, and Hand (DASH) questionnaire, ${ }^{6}$ Center for Epidemiologic Studies Depression (CESD) Scale, ${ }^{7}$ the Whiteley Index, ${ }^{8}$ and the Pain Catastrophizing Scale (PCS). ${ }^{3}$ The visit was then resumed, and as part of standard care, the surgeon coached the patient for ten minutes on the importance of stretching and how to stretch effectively. During the follow-up appointment, we again measured pain, DASH questionnaire, symptoms of depression, Whiteley Index, and Pain Catastrophizing.

\section{Outcome measures}

The Center for Epidemiologic Studies Depression (CESD) Scale consists of 20 questions answered on a 4-point Likert scale ( 0 indicating "rarely" and 3 "most of the time"), resulting in a score from 0 to 60 with a higher score indicating more depressive symptoms. ${ }^{7}$

Symptoms of hypochondriasis were measured using the Whiteley Index, a 14-question survey using a 5-point Likert scale ranging from 1, "not at all" to 5, "a great deal," resulting in a score from 14 to 70 . A higher score indicates a more heightened illness concern. ${ }^{8}$

The Pain Catastrophizing Scale (PCS) measures catastrophic thinking: an exaggerated negative attitude towards or an overinterpretation of pain. PCS uses 13 questions answered with a 5-point Likert scale ( 0 meaning "not at 
all" and 4 "all the time") for an overall score between 0 and 52, with a higher score indicating higher levels of pain catastrophizing. ${ }^{3}$

The Disabilities of the Arm, Shoulder and Hand (DASH) questionnaire is used to assess symptoms and disability of the upper extremity. The questionnaire involves 30 questions regarding the use of the arm on a 5-point Likert scale response ( 1 indicates "no problems/pain" and 5 indicates "impossible"). The final score ranges from 0 to 100 , with a higher score indicating higher levels of pain and disability in the arm. ${ }^{6}$

Two 11 -point ordinal scales were used to assess the patients' general pain levels, ranging from 0, "no pain," to 10 , "the worst pain ever,", as well as the patients' agreement with "no pain, no gain" regarding the importance of stretching to discomfort in order to recover.

\section{Patient demographics}

In total, 69 patients were enrolled (mean $5 \pm 2$ days, range 1-12 days) after injury. Of the 55 patients (80\%) returning after 1 month (mean 36 days after the first visit, range 20-84 days), 38 had proximal radius fractures and 17 had distal radius fractures. Average age was $47 \pm 16$ years (range 20-80 years), and 39 were female (Table 1).

\section{Table 1. Demographics of patients with a nondisplaced radial head fracture $(n=55)$}

$\begin{array}{ll}\text { Variable } & \text { Value } \\ \text { Age; range } & 47 \pm 16 \text { (ran } \\ \text { Female } & 71 \%(39) \\ \text { Smoking } & 11 \%(6) \\ \text { Pain condition } & 20 \%(11) \\ \text { Marital status } & \\ \text { • Single } & 42 \%(23) \\ \text { • Partner/married } & 26 \%(20) \\ \text { • Separated/widowed } & 22 \%(12) \\ \text { Employed at time of fracture } & 84 \%(46) \\ \text { Years of education } & 16 \pm 3 \\ \text { Immobilization } & 73 \%(40) \\ \text { Fracture location } & \\ \text { • Radial head fractures } & 69 \%(38) \\ \text { • Distal radius fractures } & 31 \%(17) \\ \text { Pain Score } & 5 \pm 2 \\ \text { Agreement "no pain, no gain” } & 7 \pm 3 \\ \text { DASH questionnaire enrollment } & 50 \pm 17 \\ \text { DASH questionnaire follow-up } & 20 \pm 17\end{array}$

Continuous variables as mean ( \pm standard deviation); discrete variables as percentage (number); DASH = Disabilities of the Arm, Shoulder and Hand.

\section{Statistical analysis}

Continuous variables are presented as mean ( \pm standard deviation), and discrete variables as number and 
percentage. Histograms showed the data was normally distributed, allowing the use of parametric testing. The enrollment and follow-up patient survey scores for CESD, Whiteley Index, and Pain Catastrophizing Scale were compared using paired t-tests. Association between changes in survey scores and independent variables was assessed using independent t-tests (dichotomous variables), one-way analyses of variance (categorical variables), or Pearson correlations (continuous variables). $P$ values less than 0.05 were deemed significant. A priori power analysis indicated 52 patients would yield 80\% power (alpha set at 0.05 ) to detect a difference with an effect size of 0.40 using a paired t-test.

For 4 missing CESD items we imputed the cohort's median value to calculate the composite questionnaire score. Mean imputations were used for the following missing variables: education level (once), "no pain, no gain" agreement (once), DASH scores (twice), and Whiteley Index follow-up score (once).

\section{RESULTS}

The average CESD and PCS scores decreased slightly but significantly over time (Table 2). CESD scores decreased by an average of $5 \pm 9$ points $(27 \pm 102 \%, P<0.001)$ and PCS scores decreased by $2 \pm 6$ points $(9 \pm 28 \%$, $P=0.0041)$.

\section{Table 2. Psychological Questionnaires at 1 and 5 weeks after injury}

\begin{tabular}{|llllll|}
\hline Questionnaire & $\begin{array}{l}\mathbf{1} \text { week } \\
\text { after injury }\end{array}$ & $\begin{array}{l}\mathbf{5} \text { weeks } \\
\text { after injury }\end{array}$ & P value & $\begin{array}{l}\text { Change in } \\
\text { questionnaire }\end{array}$ & $\begin{array}{l}\text { Percent } \\
\text { change }\end{array}$ \\
Center for Epidemiologic Studies Depression Scale & $12 \pm 7.7$ & $6.2 \pm 7.0$ & $<0.001$ & $-4.7 \pm 9.3$ & $-27 \pm 102 \%$ \\
Whiteley Index & $3.2 \pm 2.6$ & $2.6 \pm 1.9$ & 0.084 & $-0.56 \pm 1.9$ & $-3.4 \pm 97 \%$ \\
Pain Catastrophizing Scale & $18 \pm 5.1$ & $15 \pm 3.7$ & 0.0041 & $-2.3 \pm 5.7$ & $-9.2 \pm 28 \%$ \\
& & & & & \\
Values as mean ( \pm standard deviation); P value $<0.05$ indicates statistically significant difference.
\end{tabular}

In bivariate analysis, increase in symptoms of depression was associated with a preexisting pain condition, less time between injury and final evaluation, and lower "no pain, no gain" agreement. Accounting for potential interaction of variables using multivariable analysis, decrease in CESD was associated with not having an additional pain condition ( $\beta$-regression coefficient $[\beta] 5.8$, partial $\mathrm{R}^{2} 0.078,95 \%$ confidence interval $[\mathrm{Cl}] 0.616$ to $11, P=0.049)$, more days elapsed between injury and final evaluation $\left(\beta-0.23\right.$, partial $\mathrm{R}^{2} 0.14,95 \% \mathrm{Cl}-0.39$ to $-0.067, P=0.007)$, and stronger agreement with "no pain, no gain" $\left(\beta-1.2\right.$, partial $\mathrm{R}^{2} 0.14,95 \% \mathrm{Cl}-2.1$ to -0.35, $P=0.007$ ) (adjusted $\mathrm{R}^{20.26}, P=0.0006$ ) (Table $3 \& 4$ ). In other words, having an additional pain condition increases CESD score on average by 5.8 points; each additional day decreased the CESD score by 0.23 points; and on average, a one-point increase in agreement decreases CESD score by 1.2 points.

An increase in Whiteley Index scores was associated with less years of education $(R=-0.34 ; P=0.012)$. Changes in PCS scores were associated with marital status. Partnered or married patients showed a decrease in catastrophic thinking by an average of $5 \pm 6$ points; single patients showed an average decrease of $2 \pm 4$ points; separated or widowed patients showed an increase of $1 \pm 6$ points $(P=0.040)$.

Because no other variables were associated with changes in Whiteley Index or Pain Catastrophizing Scale, we omitted multivariable analyses. 
Table 3. Factors associated with change in questionnaire score

Change in questionnaire between 1 and 5 weeks after injury

\section{Demographic variables}

Age (range), years [r]

Sex

$$
\text { - Male }
$$$$
\text { - Female }
$$

Smoking

- Yes

- No

Pain condition

$$
\text { - Yes }
$$

- No

Marital status

- Single

- Partner/married

- Separated/widowed

Employed at time of fracture

- Yes

- No

Years of education $[r]$

Immobilization

$$
\text { - Yes }
$$$$
\text { • No }
$$

Fracture location

Agreement "no pain, no gain"

Radial head fractures

Distal radius fractures

\section{Other Variables [r]}

\begin{tabular}{|c|c|c|c|c|c|}
\hline $\begin{array}{l}\text { Center for Epidemiologic } \\
\text { Studies Depression Scale }\end{array}$ & $P$ value & Whiteley Index & $P$ value & $\begin{array}{l}\text { Pain Catastrophizing } \\
\text { Scale }\end{array}$ & $P$ value \\
\hline-0.0079 & 0.96 & -0.071 & 0.61 & 0.0047 & 0.97 \\
\hline$-5.2 \pm 8.1$ & \multirow{2}{*}{0.82} & $-0.6 \pm 1.8$ & \multirow{2}{*}{0.94} & $-2.1 \pm 3.5$ & \multirow{2}{*}{0.87} \\
\hline$-4.5 \pm 9.8$ & & $-0.55 \pm 2.6$ & & $-2.4 \pm 6.4$ & \\
\hline $0.4 \pm 16$ & \multirow{2}{*}{0.20} & $1.4 \pm 1.6$ & \multirow{2}{*}{0.051} & $-2.6 \pm 15$ & \multirow{2}{*}{0.92} \\
\hline$-5.2 \pm 8.5$ & & $-0.76 \pm 2.2$ & & $-2.3 \pm 4.1$ & \\
\hline $1.1 \pm 3.6$ & \multirow{2}{*}{0.031} & $0.14 \pm 1.0$ & \multirow{2}{*}{0.27} & $-.5 \pm 7.1$ & \multirow{2}{*}{0.26} \\
\hline$-5.9 \pm 8.4$ & & $-0.76 \pm 2.1$ & & $-2.8 \pm 5.4$ & \\
\hline$-4.63 \pm 9.2$ & \multirow{3}{*}{0.058} & $-0.5 \pm 1.5$ & \multirow{3}{*}{0.20} & $-1.7 \pm 4.3$ & \multirow{3}{*}{0.040} \\
\hline$-7.7 \pm 6.9$ & & $-1.2 \pm 2.7$ & & $-4.6 \pm 6.0$ & \\
\hline $0.55 \pm 12$ & & $0.38 \pm 2.8$ & & $0.55 \pm 6.2$ & \\
\hline $5.0 \pm 8.7$ & \multirow{2}{*}{0.55} & $0.77 \pm 2.4$ & \multirow{2}{*}{0.14} & $-2 \pm 5$ & \multirow{2}{*}{0.34} \\
\hline$-2.9 \pm 13$ & & $0.50 \pm 2.0$ & & $-4.1 \pm 8.7$ & \\
\hline-0.22 & 0.13 & -0.34 & 0.012 & -0.042 & 0.77 \\
\hline$-5.7 \pm 9.2$ & \multirow{2}{*}{0.19} & $-0.37 \pm 2.2$ & \multirow{2}{*}{0.073} & $-2.7 \pm 4.2$ & \multirow{2}{*}{0.49} \\
\hline$-1.9 \pm 9.4$ & & $-0.91 \pm 2.4$ & & $-1.4 \pm 8$ & \\
\hline-0.33 & 0.017 & 0.045 & 0.74 & -0.13 & 0.37 \\
\hline$-4 \pm 8.3$ & \multirow{2}{*}{0.42} & $0.63 \pm 2.6$ & \multirow{2}{*}{0.75} & $-2.5 \pm 6.2$ & \multirow{2}{*}{0.73} \\
\hline$-6.2 \pm 11$ & & $0.42 \pm 1.9$ & & $-1.9 \pm 4.5$ & \\
\hline-0.17 & 0.23 & 0.061 & 0.66 & 0.076 & 0.59 \\
\hline-0.31 & 0.025 & -0.034 & 0.81 & -0.18 & 0.19 \\
\hline
\end{tabular}

Days between injury and enrollment

Days between injury and final evaluation

\begin{tabular}{|c|c|c|c|c|c|}
\hline Change CESD score & $\begin{array}{l}\text { Regression coefficient } \\
\text { (95\% confidence interval) }\end{array}$ & Standard error & $\begin{array}{l}P \\
\text { value }\end{array}$ & Partial $\mathbf{R}^{2}$ & Adjusted $\mathbf{R}^{2}$ \\
\hline Having an additional pain condition & $5.8(0.16-11)$ & 2.9 & 0.049 & 0.078 & \multirow{3}{*}{0.26} \\
\hline Days between injury and final evaluation & $-0.23(-0.39--0.067)$ & 0.081 & 0.007 & 0.14 & \\
\hline "no pain, no gain" agreement & $-1.2(-2.1--0.35)$ & 0.44 & 0.007 & 0.14 & \\
\hline
\end{tabular}

P value $<0.05$ indicates statistically significant difference; continuous variables as mean ( \pm standard deviation). Pearson correlation indicated by $r$.

\section{Table 4. Multivariable analysis change in symptom of depression score between 1 and 5 weeks after injury}

P value $<0.05$ indicates statistically significant difference. CESD $=$ Center for Epidemiologic Studies Depression Scale; $W I=$ Whiteley Index 


\section{DISCUSSION}

Psychological factors are associated with increased magnitude of disability in patients with musculoskeletal illnesses.' The stability of these psychological constructs during recovery from injury is unclear. We aimed to assess change in symptoms of (1) depression, (2) health anxiety, and (3) catastrophic thinking between approximately 1 and 6 weeks after injury. We found symptoms of depression and catastrophic thinking decreased slightly but significantly.

This study has several limitations. First, we only included isolated non-operatively-treated radial head and distal radius fractures. More severe fractures requiring surgery, or multitrauma, might influence psychological constructs differently. Secondly, the treating surgeon is mindful about the psychological aspects of recovery and might use words or advice that can influence psychological constructs over time. The changes in psychology might be provider specific.

The average CESD and PCS scores decreased slightly but significantly over time. During validation in healthy individuals both scales showed good test-retest reliability. ${ }^{2,3}$ Over 2 weeks, the PCS scores were stable in patients with chronic temporomandibular pain. ${ }^{9}$ However, during recovery both measures seem to change as well, potentially to a pre-injury state. Since psychological measures are associated with increased disability, they potentially could be used as a screening tool to predict outcome after surgical treatment. This study suggests that one should account for a patient's disease phase as psychological constructs can change during recovery. In other words, symptoms of depression and ineffective coping strategies seem in part to represent a patient's baseline and in part a reaction to trauma.

Decrease in CESD was associated with not having an additional pain condition, more days elapsed between injury and final evaluation, and stronger agreement with "no pain, no gain". Those findings are in line with previous published studies. In a post hoc analysis of depression recovery in primary care patients, decrease in depressive symptoms was associated with a lack of medical comorbidity, and personal health beliefs. Patients who believed in control of their own health showed greater decreases in depressive symptoms. ${ }^{10}$ An increased agreement with "no pain, no gain" mirrors this aforementioned perception of control of health. In a similar study, patients with depression showed a greater recovery over a 12-month period than patients with both depression and other axis I, II, or II comorbidities (compound depression) did." In a study of hospitalized patients with major depression, recovery rates were not affected by demographic variables such as age, marital and socioeconomic status, and education level. The most important factors associated with depression recovery included shorter hospital stay, absence of comorbidities, older age at onset of depression, fewer previous hospitalizations, and high family functioning. ${ }^{12}$ Coaching patients after injury on mindset might improve recovery, something future research could address.

An increase in Whiteley Index scores was associated with less years of education. The understanding of the associated factors with changes in hypochondria is widely inconsistent. Comorbidities, for example, have been shown to have positive, negative and negligible impacts on hypochondria recovery. The association between patient age and hypochondria recovery is also disputed. ${ }^{13}$

Decrease in catastrophic thinking was associated with being married. Previous studies showed that married people have lower rates of chronic limitations and disability, ${ }^{14}$ and when visiting a hand surgery clinic, married patients, in general, have lower disability measured by QuickDASH (Disabilities of the Arm, Shoulder and Hand). ${ }^{15}$ Less disability tends to reflect greater adaptation and resilience, skills potentially enhanced by marriage. 
In conclusion, symptoms of depression and catastrophic thinking, but not health anxiety, improved during recovery after upper extremity injury. Symptoms of depression and ineffective coping strategies are in part reactive and can be expected to improve with time. This suggests that caregivers should be both (1) aware of the psychological aspects of recover and potential for coaching on mindset and coping strategies to improve recovery and (2) patients with the normal human response to injury. This is important because patients that are slow to master stretching exercises and return to daily activities are often considered in need of additional medical treatment, when empathy, support, and patience might suffice. 


\section{REFERENCES}

1. Vranceanu AM, Bachoura A, Weening A, Vrahas M, Smith RM, Ring D. Psychological factors predict disability and pain intensity after skeletal trauma. J Bone Joint Surg Am. 2014;96(3):e20.

2. Miller WC, Anton HA, Townson AF. Measurement properties of the CESD scale among individuals with spinal cord injury. Spinal Cord. 2008;46(4):287-292.

3. Sullivan MJL, Bishop SR, Pivik J. The Pain Catastrophizing Scale: Development and validation. Psychological Assessment. 1995;7(4):524-532.

4. Stewart SH, Watt MC. Assessment of health anxiety. In: Asmundson GJG, Taylor S, Cox BJ, eds. Health anxiety: Clinical and research perspectives on hypochondriasis and related conditions. New York: Wiley; 2001:95-131.

5. Mossey JM, Mutran E, Knott K, Craik R. Determinants of recovery 12 months after hip fracture: the importance of psychosocial factors. Am J Public Health. 1989;79(3):279-286.

6. Hudak PL, Amadio PC, Bombardier C. Development of an upper extremity outcome measure: the DASH (disabilities of the arm, shoulder and hand) [corrected]. The Upper Extremity Collaborative Group (UECG). Am J Ind Med. 1996;29(6):602608.

7. Barlow JH, Wright CC. Dimensions of the Center of Epidemiological Studies-Depression Scale for people with arthritis from the UK. Psychol Rep. 1998;83(3 Pt 1):915-919.

8. Pilowsky I. Dimensions of hypochondriasis. Br J Psychiatry. 1967;1 13(494):89-93.

9. Turner JA, Mancl L, Aaron LA. Pain-related catastrophizing: a daily process study. Pain. 2004;1 10(1-2):103-111.

10. Brown C, Schulberg HC, Prigerson HC. Factors associated with symptomatic improvement and recovery from major depression in primary care patients. Gen Hosp Psychiatry. 2000;22(4):242-250.

11. Keitner GI, Ryan CE, Miller IW, Kohn R, Epstein NB. 12-month outcome of patients with major depression and comorbid psychiatric or medical illness (compound depression). Am J Psychiatry. 1991;148(3):345-350.

12. Keitner GI, Ryan CE, Miller IW, Norman WH. Recovery and major depression: factors associated with twelve-month outcome. Am J Psychiatry. 1992;149(1):93-99.

13. Olatunji BO, Kauffman BY, Meltzer S, Davis ML, Smits JA, Powers MB. Cognitive-behavioral therapy for hypochondriasis/ health anxiety: a meta-analysis of treatment outcome and moderators. Behav Res Ther. 2014;58:65-74.

14. Verbrugge LM. Marital status and health. Journal of Marriage and Family. 1979;41:267-285.

15. Janssen SJ, Ter Meulen DP, Nota SP, Hageman MG, Ring D. Does Verbal and Nonverbal Communication of Pain Correlate With Disability? Psychosomatics. 2014 


\section{CHAPTER}

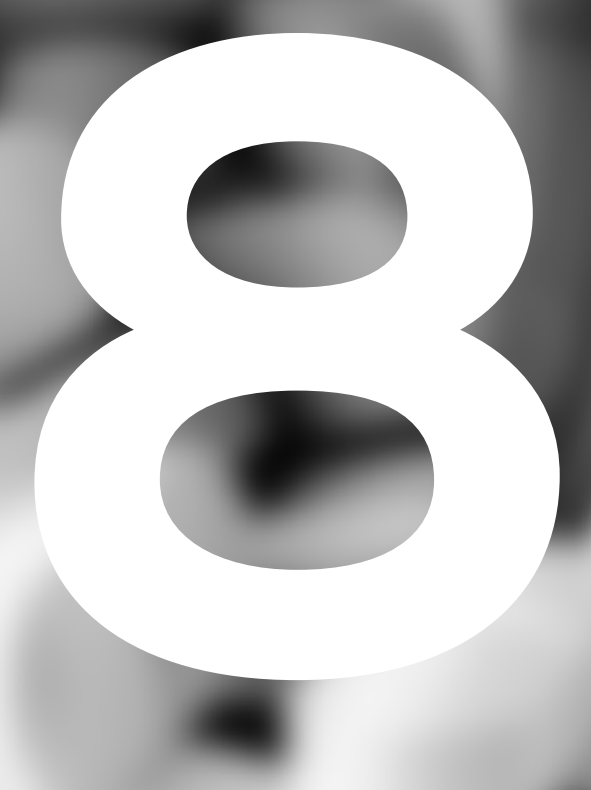




\title{
What factors are associated
}

\section{with a second opioid prescription} after treatment of distal radius fractures with a volar locking plate?

\author{
T. Teunis \\ N. Stoop \\ C.J. Park \\ D. Ring
}


Importance Knowledge of factors associated with patient's requests for a second opioid prescription after volar plate fixation of a fracture of the distal radius might inform better pain management protocols and encourage decreased and safer use of opioids.

Objectives To determine the difference in demographics, prior opioid prescriptions, injury characteristics, and psychological factors between patients that do and do not receive a second opioid prescription following treatment volar locking plate after distal radius fracture.

Design Secondary analysis of two prospective cohort study.

Setting Level I Academic Urban Trauma Center.

Participants 206 patients who underwent volar locking plate fixation of their distal radius fracture of whom 47 (23\%) received a second opioid prescription within 30 days after surgery.

Predictor variables Demographics, AO fracture type, American Society for Anesthesiologists (ASA) classification, radiographic parameters at the time of injury prior to reduction and after surgery, and catastrophic thinking.

Main Outcomes Receiving a second opioid prescription within 30 days after surgery.

Results Male sex (odds ratio [OR] 2.2, 95\% confidence interval [CI] 1.0-4.6, partial pseudo $\mathrm{R}^{2}=0.018, P=0.044$ ) and greater dorsal angulation of the articular surface on the lateral post injury radiograph $(\mathrm{OR} 0.98,95 \% \mathrm{Cl}$ 0.96 to 1.0 , partial pseudo $\mathrm{R}^{2}=0.033, P=0.040$ ) were associated with a second opioid prescription after surgery (pseudo $\mathrm{R}^{2} 0.12, P=0.0071$ ).

Conclusion One measure of fracture severity (dorsal displacement) was independently associated with a second opioid prescription, but alone it accounted for 3.3\% of the variation. Other factors such as the patient's expectation prior to surgery, in particular the realization that injury and surgery hurt, might be addressed in future research.

Level of Evidence Prognostic level II 


\section{INTRODUCTION}

There is substantial variation in the amount of opioids consumed by patients after orthopaedic surgery. ${ }^{1,2}$ In 2004 it was estimated that the United States accounted for $85 \%$ of the world's oxycodone consumption and $99 \%$ of its hydrocodone consumption. ${ }^{3}$ Most patients have acceptable pain relief with acetaminophen or tramadol after orthopaedic surgery in other parts of the world. ${ }^{1,4,5}$

Patients who take more opioids after fracture treatment report greater pain intensity and less satisfaction with pain relief, both in the immediate postoperative period ${ }^{4,6}$ and 1-2 months after surgery. ${ }^{7}$ One might expect patients with injuries to larger bones, certain anatomic areas, more than one fracture, or specific procedures to have greater pain and use more opioids, but that was not the case. ${ }^{4,6}$ Previous research also found an association between greater postoperative opioid use and psychological factors, catastrophic thinking and health anxiety in particular. 7,8

Previous research found that most patients prescribed opioids during recovery from operative treatment of a fracture of the distal radius stop taking opioids within a few days. ${ }^{2}$ Knowledge of the factors associated with a second opioid prescription might inform better pain management protocols and encourage decreased and safer use of opioids after orthopaedic surgery. In particular we were interested in ineffective coping strategies such as catastrophic thinking, characteristics that may be amenable to coaching. ${ }^{9,10}$ This study tested the primary null hypothesis that there is no difference in demographics, prior opioid prescriptions, injury characteristics, and psychological factors between patients that do and do not receive a second opioid prescription following treatment of their distal radius fracture with a volar locking plate. Additionally we assessed factors associated with disability and pain measured at suture removal.

\section{PATIENTS \& METHODS}

Study design

After institutional review board approval for secondary use of the data, we reviewed 220 adult patients treated with a volar locking plate after distal radius fracture who were recruited for two previous prospective studies. One randomized controlled trial $(n=94)$ compared formal occupational therapy with instructions for independent exercises ${ }^{11}$; the other observational cohort study $(n=116)$ addressed factors associated with finger stiffness. ${ }^{12}$ Exclusion criteria for both studies were: (1) treatment more than 4 weeks after trauma; (2) inability to complete enrollment forms due to any mental status or language problems (e.g. dementia, head injury, overall illness); (3) pre-injury lack of near-normal finger motion of the uninjured hand; (4) additional injuries except ulna fractures.

\section{Outcome measures}

At suture removal, after informed consent, a researcher not involved in patient care recorded the patient's age, sex, body mass index, tobacco use, carpal tunnel release at the time of surgery, days between injury and surgery, and if the injury involved the dominant hand. AO fracture type was recorded the time of surgery (extra-, partial-, or complete articular). We extracted patients' American Society for Anesthesiologists (ASA) classification from the anesthesiology reports, and recorded the treating surgeon. We also measured the following radiographic parameters at the time of injury prior to reduction and after surgery: (1) ulnarward inclination; (2) ulnar variance; (3) volar tilt; (4) ulna intact. ${ }^{13}$ Patients completed the pain catastrophizing scale, a measure of misinterpretation or overinterpretation of nociception (catastrophic thinking). This questionnaire 
comprises 13 items, scored on a 4-point Likert scale, ranging from 1 (not at all) to 4 (all the time). The total score ranges from 13-52 points with a higher score indicating greater catastrophic thinking. ${ }^{14}$ Arm specific disability was evaluated by the Disabilities of the Arm, Shoulder and Hand (DASH) questionnaire. It consists of 30 questions scored on 5-point Likert scales, ranging from 1 (no problems/pain) to 5 (impossible). Scores range between 0 and 100 points, a higher score indicating worse upper extremity specific disability and pain. ${ }^{15}$ Patients rated their pain intensity on an 11 -point ordinal scale, ranging from 0 to 10 , where 0 was no pain and 10 the worst pain ever. ${ }^{16}$

From the pharmacy records and the patient charts we extracted the type of opioid prescribed, the dosage, and the number of pills. We also had records of a second opioid prescription within 30 days after surgery and all opioids prescribed 90 days prior to surgery. We divided this period in 4 time frames: (1) opioids prescribed up to 90 days before fracture; (2) opioids prescribed between fracture and 4 days before surgery; (3) opioids prescribed up to 3 days before surgery (peri-operative opioids); (4) any opioid prescription in addition to the first opioid prescription given post-operatively up to 30 days after surgery. Medication with additional acetaminophen or non-steroid anti-inflammatory drugs was grouped with its type of opioid (e.g., vicodin and norco with hydrocodone). Extended release compositions were sorted with the main opioid group (e.g., oxcontin grouped with oxycodone). Using equianalgesia conversion factors ${ }^{17-19}$ we changed all opioids to oral morphine equivalent dosages (Appendix 1). Subsequently we calculated the prescribed morphine dosage during the 4 time periods.

\section{Study population}

We excluded 14 patients because they were initially treated at another hospital and we could not track their prescribed opioid medication. Our final cohort included 206 patients, of whom 60 (30\%) were men. The mean age was 53 years ( \pm SD [standard deviation] 15, range 19-89) (Table 1). A second opioid prescription was provided to 47 (23\%) patients; mean oral morphine equianalgesia dosage prescribed was $244 \mathrm{mg}( \pm$ SD 77 , range 100-450) (Table 2). The majority of patients were treated by one of three surgeons, surgeon $A$ operated 155 (75\%) patients, surgeon B 19 (9\%) patients, and surgeon C 14 (7\%) patients; 8 other surgeons together operated the remaining 18 patients (9\%) (Table 1).

\begin{tabular}{ll}
$\begin{array}{l}\text { Table 1. Baseline characteristics of patients with a } \\
\text { distal radius fracture treated with a volar locking } \\
\text { plate }\end{array}$ \\
$\begin{array}{ll}\text { Demographics } & \text { Value } \\
\text { Patients } & 206 \\
\text { Age, years (range) } & 53 \pm 15(19-89) \\
\text { Male } & 30 \%(62) \\
\text { Body mass index } & 26 \pm 6.0 \\
\text { Smoking } & 8.9 \%(17) \\
\text { ASA classification } & \\
\cdot 1 & 36 \%(73) \\
.2 & 58 \%(117) \\
.3 & 5.9 \%(12)\end{array}$ \\
\hline
\end{tabular}

Table 2. Opioid related factors of patients with a distal radius fracture treated with a volar locking plate

\begin{tabular}{|ll|}
\hline Opioid related factors & Value \\
Opiods within 90 days prior to injury & $5 \%(10)$ \\
. Oral morphine equianalgesia $(\mathrm{mg})$ & $2725 \pm 5106$ \\
Opioids between injury \& surgery & $44 \%(91)$ \\
. Oral morphine equianalgesia $(\mathrm{mg})$ & $309 \pm 233$ \\
& \\
Opioids peri-operative & $81 \%(167)$ \\
. Oral morphine equianalgesia (mg) & $470 \pm 215$
\end{tabular}




\begin{tabular}{|c|c|}
\hline \multicolumn{2}{|l|}{ Treating surgeon } \\
\hline$\cdot A$ & $75 \%(155)$ \\
\hline$\cdot B$ & $9.2 \%(19)$ \\
\hline$\cdot C$ & $6.8 \%(14)$ \\
\hline • Other & $8.7 \%(18)$ \\
\hline \multicolumn{2}{|l|}{ Trauma related factors } \\
\hline Injury to dominant side & $43 \%(88)$ \\
\hline Carpal tunnel release during ORIF & $18 \%(38)$ \\
\hline \multicolumn{2}{|l|}{ A0 classification } \\
\hline$\cdot A$ & $41 \%(84)$ \\
\hline$\cdot B$ & $13 \%(26)$ \\
\hline$\cdot C$ & $47 \%(96)$ \\
\hline Ulna fracture & $58 \%(119)$ \\
\hline Days between injury and surgery & $8.8 \pm 5.8$ \\
\hline \multicolumn{2}{|l|}{ Radiographic parameters after injury } \\
\hline - Ulnarward inclination & $12 \pm 8.5^{\circ}$ \\
\hline - Ulnar variance & $1.4 \pm 4.7 \mathrm{~mm}$ \\
\hline - Volar tilt & $-13 \pm 21^{\circ}$ \\
\hline \multicolumn{2}{|c|}{ Radiographic parameters after surgery } \\
\hline - Ulnarward inclination & $20 \pm 4.6^{\circ}$ \\
\hline - Ulnar variance & $-0.21 \pm 3.0 \mathrm{~mm}$ \\
\hline - Volar tilt & $5.9 \pm 8.2^{\circ}$ \\
\hline \multicolumn{2}{|l|}{ Questionnaires } \\
\hline Pain catastrophizing scale & $18 \pm 6.9$ \\
\hline DASH score & $48 \pm 19$ \\
\hline Numerical rating of pain intensity & $3.6 \pm 2.6$ \\
\hline \multicolumn{2}{|c|}{$\begin{array}{l}\text { ASA = American Society for Anesthesiologists; DASH = Disability of the } \\
\text { Arm, Shoulder and Hand. Continuous variables as mean } \pm \text { standard } \\
\text { deviation; discrete data as percentage (number). }\end{array}$} \\
\hline
\end{tabular}

\begin{tabular}{|ll} 
Second opioid prescription & $23 \%(47)$ \\
• Oral morphine equianalgesia (mg) & $244 \pm 77$ \\
Types of opioid prescribed up to surgery & \\
• Oxycodone & $84 \%(174)$ \\
• Hydrocodone & $28 \%(58)$ \\
• Hydromorphone & $3.9 \%(8)$ \\
• Codeine & $3.9 \%(8)$ \\
• Tramadol & $2.4 \%(5)$ \\
• Propoxyphene & $1.9 \%(4)$ \\
• & \\
• Procond opioid prescriptions (n=47) & \\
• Oxycodone $5 \mathrm{mg}$ & \\
• Hydrocodone $5 \mathrm{mg}$ & $13 \%(6)$ \\
• Hydrocodone $7.5 \mathrm{mg}$ & $68 \%(32)$ \\
\hline
\end{tabular}

Continuous variables as mean \pm standard deviation; discrete data as percentage (number).

\section{Statistical analysis}

To identify independent factors associated with (1) additional opioid prescription, (2) disability and (3) pain we created three multivariable models. The potential explanatory variables associated with our outcome measurements were selected based on the feasibility of measurement in the clinical setting and their possible influence on a second opioid prescription. Multiple logistic and linear regression models were created by entering catastrophic thinking (our primary explanatory variable) in addition to all other variables associated with each of the three response variables on exploratory bivariate analysis with $P<0.10$ (see bivariate analysis in Appendix 2 \& 3). In case of significant association with both morphine equianalgesia dosage and opioid prescription, we only included equianalgesia dosage in our model due to covariance of both factors. Pseudo and adjusted $R^{2}$ indicate how much variability in the outcome variable the model accounts for. The partial $R^{2}$ 
indicates for how much variability each variable accounts for by itself.

We used multiple linear imputation for missing values (number of imputations set to 40): 15 tobacco use (7.3\%), 4 ASA classification (1.9\%), 56 volar tilt after injury (27\%), 3 ulnar variance after surgery (1.5\%), 2 volar tilt after surgery (0.97\%), 6 pain scores (2.9\%), and 20 DASH scores (9.7\%). All $R^{2}$ are the average of the 40 imputed sets.

Continuous variables are described as mean $( \pm S D)$, discrete variables as percentage and number. Data histograms were visually inspected to assess data distribution. Accordingly we compared continuous and discrete variables by unpaired Student t-test or analysis of variance, continous variables by Pearson correlation and discrete variables by Fisher exact test. Bivariate analysis was performed only on complete data.

We considered a two-sided $P$ value of less than 0.05 significant; all statistical analyses were performed using Stata 13.0 (StataCorp LP, Texas, USA).

A priori power analysis for a multiple logistic regression analysis, including catastrophic thinking as our key predictor of additional opioid prescription, was based on a pilot dataset of 108 patients. The probability of additional opioid prescription was 0.33 (2 of 6 ) at the mean catastrophic thinking of 17 . Probability increased to 0.50 ( 2 of 4 ) at an increase of 1 standard deviation in catastrophic thinking (standard deviation 5.9).

Assuming a moderate squared multiple correlation of 0.40 between catastrophic thinking and other predictors in the model, power analysis for a multiple logistic regression with multiple predictors indicated 181 patients would provide 0.90 power with alpha set at 0.05 (powerlog command, Stata 13.0, StataCorp LP, Texas, USA).

\section{RESULTS}

Opioids

Accounting for potential interaction of variables using multivariable analysis, male sex (odds ratio [OR] 2.2, 95\% confidence interval $[\mathrm{Cl}]$ 1.0-4.6, partial pseudo $\mathrm{R}^{2}=0.018, P=0.044$ ) and greater dorsal angulation of the articular surface on the lateral post injury radiograph $\left(\mathrm{OR} 0.98,95 \% \mathrm{Cl} 0.96\right.$ to 1.0 , partial pseudo $\mathrm{R}^{2}=0.033$, $P=0.040$ ) were associated with a second opioid prescription after surgery (pseudo $\mathrm{R}^{2} 0.12, P=0.0071$ ), but not catastrophic thinking. The odds of a second opioid prescription were 2.2 times higher in male patients. The odds of a second prescription increased with $2 \%$ with every degree of less volar angulation of the articular surface after injury (Table 3).

\section{DASH score}

Higher DASH scores were independently associated with ASA class 2 ( $\beta$ regression coefficient $[\beta] 5.6,95 \% \mathrm{Cl}$ 0.57 to 11 , SE 2.6, partial $\left.\mathrm{R}^{2}=0.014, P=0.029\right)$, injury to the dominant side $(\beta 8.8,95 \% \mathrm{Cl} 4.1$ to 13 , SE 2.4 , partial $\left.\mathrm{R}^{2}=0.048, P<0.001\right)$, and greater catastrophic thinking $\left(\beta 1.1,95 \% \mathrm{Cl} 0.78\right.$ to $1.5, \mathrm{SE} 0.18$, partial $\mathrm{R}^{2}$ $=0.14, P<0.001$ ) (adjusted $\mathrm{R}^{2} 0.33, P<0.001$ ). The $\beta$ regression coefficient indicates that patients with ASA class 2 on average have 5.4 points higher DASH scores compared to patients with ASA class 1 . Patients with an injury to the dominant hand have 9.9 points higher DASH scores. Also, every point increase in catastrophic thinking on average results in a 1.1 point higher DASH score (Table 3 ). 


\section{Pain intensity}

More pain was independently associated with ASA class $2\left(\beta 0.78,95 \% \mathrm{Cl} 0.068\right.$ to $1.5, \mathrm{SE} 0.36$, partial $\mathrm{R}^{2}=$ $0.014, P=0.032)$, carpal tunnel release at the time of plate fixation $(\beta 0.91,95 \% \mathrm{Cl} 0.046$ to $1.8, \mathrm{SE} 0.44$, partial $\left.\mathrm{R}^{2}=0.015, P=0.039\right)$, greater dorsal angulation of the articular surface on the lateral post surgery radiograph $\left(\beta-0.042,95 \% \mathrm{Cl}-0.084\right.$ to 0.0011 , SE 0.021 , partial $\left.\mathrm{R}^{2}=0.014, P=0.044\right)$, and greater catastrophic thinking ( $\beta 0.12,95 \% \mathrm{Cl} 0.060$ to 0.17 , SE 0.028, partial $\left.\mathrm{R}^{2}=0.073, P<0.001\right)$ (adjusted $\mathrm{R}^{2} 0.17, P<0.001$ ). The $\beta$ regression coefficient indicates that patients with ASA class 2 on average have 0.78 points higher pain scores compared to ASA class 1 patients. Patients undergoing additional carpal tunnel release have 0.91 point higher pain scores. Pain score increases 0.042 points with every degree of less volar angulation of the articular surface after surgery. Every point increase in catastrophic thinking on average results in 0.12 higher pain scores (Table 3).

\section{Table 3. Multivariable analyses of factors associated with an additional opioid prescription, disability, and pain after distal radius fracture surgery}

\section{Second opioid prescription}

Male sex

ASA classificationt

$\cdot 1$

$\cdot 2$

$\cdot 3$

Oral morphine equianalgesia within 90 days prior to injury

Oral morphine equianalgesia between injury \& surgery

Ulna fracture

Volar tilt after injury $\dagger$

Pain Catastrophizing Scale

\section{DASH score}

Smoking†

ASA classification $†$

$\cdot 1$

$\cdot 2$

$\cdot 3$

Surgeon

-A

- $B$

- $\mathrm{C}$

- Other

Oxycodone

Propoxyphene

Injury to dominant side

Ulnar variance after surgery

Pain Catastrophizing Scale

\section{Odds ratio ( $95 \%$ confidence interval)}

$2.2(1.0-4.6)$

reference value

$1.1(0.50-2.4)$

$3.0(0.72-12)$

$1.0004(0.9995-1.001)$

$1.0008(0.9992-1.002)$

$2.1(0.94-4.5)$

$0.98(0.96-1.0)$

$1.02(0.97-1.1)$

$\beta$ Regression coefficient ( $95 \%$ confidence interval)

$2.4(-6.2-11)$

reference value

$5.6(0.57-11)$

$2.0(-9.6-14)$

reference value

$-4.9(-13-2.8)$

$-5.8(-15-3.1)$

$5.7(-2.3-14)$

$-5.4(-12-1.6)$

$9.2(-7.4-26)$

$8.8(4.1-13)$

$-0.73(-1.5-0.065)$

$1.1(0.78-1.5)$
0.83

0.044

Partial Pseudo $\mathbf{R}^{2}$ Pseudo $\mathbf{R}^{2}$

0.018

$\begin{array}{lll}0.43 & 0.83 & \\ 2.1 & 0.13 & \\ 0.00048 & 0.35 & \\ & & \\ 0.00077 & 0.33 & \\ 0.82 & 0.072 & \\ 0.010 & 0.040 & 0.033 \\ 0.027 & 0.47 & \end{array}$

\section{Standard error Pvalue Partial $\mathbf{R}^{2}$}

0.58

4.4

$\begin{array}{lll}2.6 & 0.029 & 0.014 \\ 5.9 & 0.74 & \end{array}$




\begin{tabular}{|c|c|c|c|c|c|}
\hline \multicolumn{6}{|l|}{ Pain intensity } \\
\hline Smokingt & $0.26(-1.0-1.5)$ & 0.64 & 0.68 & & \\
\hline \multicolumn{6}{|l|}{ ASA classificationt } \\
\hline 1 & reference value & & & & \\
\hline 2 & $0.78(0.068-1.5)$ & 0.36 & 0.032 & 0.014 & \\
\hline 3 & $0.47(-1.1-2.1)$ & 0.80 & 0.56 & & \\
\hline \multicolumn{6}{|l|}{ Oral morphine equianalgesia (mg) } \\
\hline Prior to injury & $0.00026\left(-6.88^{*} 10^{-6}-0.00053\right)$ & 0.00014 & 0.056 & & 0.17 \\
\hline Between injury \& surgery & $0.00067(-0.00089-0.0022)$ & 0.00079 & 0.40 & & \\
\hline Propoxyphene & $2.2(-0.20-4.7)$ & 1.2 & 0.071 & & \\
\hline Carpal tunnel release during ORIF & $0.91(0.046-1.8)$ & 0.44 & 0.039 & 0.015 & \\
\hline Volar tilt after surgery $\dagger$ & $-0.042(-0.084--0.0011)$ & 0.021 & 0.044 & 0.014 & \\
\hline Pain Catastrophizing Scale & $0.12(0.060-0.17)$ & 0.028 & $<0.001$ & 0.073 & \\
\hline
\end{tabular}

\section{DISCUSSION}

Patients who take more opioids after fracture treatment report greater pain intensity and less satisfaction with pain relief. ${ }^{4,6,7}$ Knowledge of the factors associated with greater opioid use might inform better pain management protocols and encourage decreased and safer use of opioids after orthopaedic surgery. We aimed to identify factors associated with a second opioid prescription after distal radius fracture surgery.

This study has some limitations. First, we were only able to track opioid prescriptions 90 days prior to injury prescribed by physicians at our hospital. Our study cannot account for opioids prescribed by outside providers. Secondly, we didn't measure the number of pills actually taken; instead we used a second opioid prescription as a surrogate measure. Thirdly, we only had complete data on 149 patients, mainly because volar tilt after injury could not be determined in 56 (27\%) patients. Deleting missing cases would result in a large loss of data. Instead, we addressed this by multiple linear imputation, which maintains the overall variability in the data while preserving relationships with other variables. Nonetheless this decreases reliability of volar tilt after injury as a factor in our multivariable models. Fourthly, our secondary outcome measures (disability and pain) were assessed at two weeks after surgery and only apply to short follow-up times. Results cannot be extrapolated to long-term outcomes. Finally, most of the patients come from a single practice with a strict opioid policy (20 5mg oxycodone with acetaminophen pills after surgery, a second script for hydrocodone $5 \mathrm{mg}$ with acetaminophen after office evaluation, then no more opioids). Therefore, the findings of the study may not apply to the average surgeon and average patient in other setting, particularly in the United States where opioids are often prescribed for pain.

Male sex and greater dorsal angulation of the articular surface on the lateral post injury radiograph were associated with a second opioid prescription after surgery but together only accounted for $12 \%$ of the variation in second opioid prescriptions. One measure of fracture severity (dorsal displacement) was independently associated with a second opioid prescription, but alone it accounted for $3.3 \%$ of the variation. We may be able to limit opioid use in recent, frequent, or ongoing opioid users via a combination of pre-operative preparation, close coordination with the patient's other caregivers (a patient should receive opioids from only one caregiver 
at a time), and strict policies regarding the number, type, and timing of opioid prescription. These ideas merit additional study. Our findings are in line with previous published studies that note a higher postoperative opioid consumption in men ${ }^{20,21}$, and patients who used opioids preoperatively. ${ }^{21}$ The reason for a higher opioid consumption in men is unclear and might be related to a difference in effectiveness, ${ }^{22}$ but is also ascribed to sex differences in fear of addiction, previous pain experience, and tolerance to postoperative pain and opioid side-effects. ${ }^{20} \mathrm{~A}$ previous study found that the main factor associated with opioid use one to two months after musculoskeletal trauma was greater catastrophic thinking. ${ }^{7}$ We did not find an effect of catastrophic thinking on second opioid prescriptions after distal radius fracture surgery, perhaps because the surgeons involved are quite strict with opioids and tend to identify and coach catastrophic thinking. Catastrophic thinking can manifest verbally (e.g. “unbearable”, "excruciating”, “it just won’t go”, etc.) or non-verbally (e.g. carrying the hand as if it was detached, flinching or retracting, bending rather than extending the wrist when trying to make a fist, etc.). ${ }^{23}$ It is coached primarily by acknowledging it as a normal, "programmed" human response to pain (protect, prepare for the worst), empathizing how difficult and counterintuitive the stretching exercises can be, being patient with the process, and encouraging patients to do things that are meaningful and important to them (e.g. a golfer should putt, a swimmer should swim, a knitter should knit). Another factor that might relate to a second opioid prescription is the patient's expectation prior to surgery, in particular the realization that injury and surgery hurt. Previous study also showed that greater opioid intake is culturally mediated. 4,5 These factors might be addressed in future research.

Greater symptoms and disability (higher DASH scores) were most strongly associated with catastrophic thinking. A previous study, assessing 84 patients after distal radius fracture at least 6 months after surgery, found no association of DASH scores with radiographic parameters after surgery (ulnar variance, ulnarward inclination, palmar tilt, articular surface incongruity, osteoarthritis). Conversely, we did find an association of DASH scores with injury to the dominant side, which might be due to our early assessment at suture removal and subsequent patient adaptation. Two other studies also found an association between greater catastrophic thinking and more disability after musculoskeletal trauma in general ${ }^{24}$, and after distal radius fracture surgery in particular. ${ }^{25}$ Other non-injury related factors previously associated with greater disability are injury compensation and lower level of education; ${ }^{26}$ two factors our study did not measure (very few of our patients were injured at work). The lack of correlation between radiographic measures and disability may reflect the fact that all fractures were treated operatively, with the result that substantial residual malalignment was unusual. Nevertheless, the collective data to date emphasize the influence of other factors in addition to greater pathophysiology (e.g. displacement, fracture type) on disability at suture removal after distal radius fracture. While factors indicating a more severe injury (carpal tunnel release at the time of plate fixation and greater dorsal angulation of the articular surface on the lateral radiograph) were independently associated with greater pain intensity, the strongest determinant of pain intensity was greater catastrophic thinking. The evidence that psychological factors (depression, pain anxiety and greater catastrophic thinking) are strongly associated with pain intensity after musculoskeletal trauma is compelling. ${ }^{6,24,27}$ These aspects of the human illness experience are amendable to cognitive behavioral therapy. Additional study of the use of cognitive behavioral therapy to aid recovery from fracture of the distal radius is warranted. ASA classification measures the severity of preoperative comorbidities and was associated with greater pain intensity after surgery. Greater pain intensity may be due to the pre-existing comorbidities, rather than the surgery itself. Future study should also measure pre-operative comorbidities. Until then the relevance of this finding is unclear. 
In a setting where the surgeons are cautious and strict with opioid medication (only $23 \%$ of patients received a second opioid prescription), a second opioid prescription after distal radius fracture surgery was not associated with greater catastrophic thinking even though catastrophic thinking was the factor most strongly associated with greater pain intensity. Considered along with the studies finding that opioid use is not associated with less pain or greater satisfaction with pain relief, it may be that pre-operative and postoperative teaching, coaching, and reassurance along with limited use of opioid medication are a successful pain management strategy. 


\section{REFERENCES}

1. Lindenhovius AL, Helmerhorst GT, Schnellen AC, Vrahas M, Ring D, Kloen P. Differences in prescription of narcotic pain medication after operative treatment of hip and ankle fractures in the United States and The Netherlands. J Trauma. 2009;67(1):160-164.

2. Rodgers J, Cunningham K, Fitzgerald K, Finnerty E. Opioid consumption following outpatient upper extremity surgery. J Hand Surg Am. 2012;37(4):645-650.

3. International Narcotics Control Board. Report of the International Narcotics Control Board for 2005. New York: United Nations publication; 2006.

4. Helmerhorst GT, Lindenhovius AL, Vrahas M, Ring D, Kloen P. Satisfaction with pain relief after operative treatment of an ankle fracture. Injury. 2012;43(11):1958-1961.

5. Carragee EJ, Vittum D, Truong TP, Burton D. Pain control and cultural norms and expectations after closed femoral shaft fractures. Am J Orthop (Belle Mead NJ). 1999;28(2):97-102.

6. Bot AG, Bekkers S, Arnstein PM, Smith RM, Ring D. Opioid use after fracture surgery correlates with pain intensity and satisfaction with pain relief. Clin Orthop Relat Res. 2014;472(8):2542-2549.

7. Helmerhorst GT, Vranceanu AM, Vrahas M, Smith M, Ring D. Risk factors for continued opioid use one to two months after surgery for musculoskeletal trauma. J Bone Joint Surg Am. 2014;96(6):495-499.

8. Ip HY, Abrishami A, Peng PW, Wong J, Chung F. Predictors of postoperative pain and analgesic consumption: a qualitative systematic review. Anesthesiology. 2009;111(3):657-677.

9. Wertli MM, Burgstaller JM, Weiser S, Steurer J, Kofmehl R, Held U. Influence of catastrophizing on treatment outcome in patients with nonspecific low back pain: a systematic review. Spine (Phila Pa 1976). 2014;39(3):263-273.

10. Wertli MM, Eugster R, Held U, Steurer J, Kofmehl R, Weiser S. Catastrophizing-a prognostic factor for outcome in patients with low back pain: a systematic review. Spine J. 2014;14(11):2639-2657.

11. Souer JS, Buijze G, Ring D. A prospective randomized controlled trial comparing occupational therapy with independent exercises after volar plate fixation of a fracture of the distal part of the radius. J Bone Joint Surg Am. 201 1;93(19):17611766.

12. Teunis T, Bot AG, Thornton ER, Ring D. Catastrophic Thinking is Associated with Finger Stiffness After Distal Radius Fracture Surgery. J Orthop Trauma. 2015.

13. Medoff RJ. Essential radiographic evaluation for distal radius fractures. Hand Clin. 2005;21(3):279-288.

14. Sullivan MJL, Bishop SR, Pivik J. The Pain Catastrophizing Scale: Development and validation. Psychological Assessment. 1995;7(4):524-532.

15. Hudak PL, Amadio PC, Bombardier C. Development of an upper extremity outcome measure: the DASH (disabilities of the arm, shoulder and hand) [corrected]. The Upper Extremity Collaborative Group (UECG). Am J Ind Med. 1996;29(6):602608.

16. Bijur PE, Silver W, Gallagher EJ. Reliability of the visual analog scale for measurement of acute pain. Acad Emerg Med. 2001;8(12):1153-1157.

17. Foley KM. The treatment of cancer pain. N Engl J Med. 1985;313(2):84-95.

18. Gammaitoni AR, Fine P, Alvarez N, McPherson ML, Bergmark S. Clinical application of opioid equianalgesic data. Clin J Pain. 2003;19(5):286-297.

19. Lee CR, McTavish D, Sorkin E. Tramadol. Drugs. 1993;46(2):313-340.

20. Miaskowski C, Levine JD. Does opioid analgesia show a gender preference for females? Pain Forum. 1999;8(1):34-44.

21. Ng B, Dimsdale JE, Rollnik JD, Shapiro H. The effect of ethnicity on prescriptions for patient-controlled analgesia for postoperative pain. Pain. 1996;66(1):9-12.

22. Sarton E, Olofsen E, Romberg R, et al. Sex differences in morphine analgesia: an experimental study in healthy volunteers. Anesthesiology. 2000;93(5):1245-1254; discussion 1246A.

23. Bot AG, Vranceanu AM, Herndon JH, Ring DC. Correspondence of patient word choice with psychologic factors in patients with upper extremity illness. Clin Orthop Relat Res. 2012;470(11):3180-3186.

24. Vranceanu AM, Bachoura A, Weening A, Vrahas M, Smith RM, Ring D. Psychological factors predict disability and pain intensity after skeletal trauma. J Bone Joint Surg Am. 2014;96(3):e20.

25. Roh YH, Lee BK, Noh JH, Oh JH, Gong HS, Baek CH. Effect of anxiety and catastrophic pain ideation on early recovery after surgery for distal radius fractures. J Hand Surg Am. 2014;39(11):2258-2264 e2252.

26. MacDermid JC, Donner A, Richards RS, Roth JH. Patient versus injury factors as predictors of pain and disability six months after a distal radius fracture. J Clin Epidemiol. 2002;55(9):849-854.

27. Souer JS, Lozano-Calderon SA, Ring D. Predictors of wrist function and health status after operative treatment of fractures of the distal radius. J Hand Surg Am. 2008;33(2):157-163. 


\section{Appendix 1. Equianalgesia conversion factor}

\section{Opioid}

Hydromorphone [18]

Oxycodone [18]

Propoxyphene [17]

Hydrocodone [18]

Tramadol [19]

Codeine [18]

\section{Equianalgesia dosage of $10 \mathrm{mg}$ oral morphine}

$2.5 \mathrm{mg}$

$6.7 \mathrm{mg}$

$0.74 \mathrm{mg}$

$10 \mathrm{mg}$

$100 \mathrm{mg}$

$67 \mathrm{mg}$

\section{Appendix 2. Bivariate analysis of factors associated with an additional opioid prescription}

\section{after distal radius fracture surgery}

Demographics
Patients
Age
Male
Body mass index
Smoking
ASA classification
1
2
3

Treating surgeon

$$
\begin{aligned}
& \text { A } \\
& \text { B }
\end{aligned}
$$$$
\text { C }
$$$$
\text { Other }
$$

\section{Opioid related factors}

Opioids within 90 days prior to injury Oral morphine equianalgesia $(\mathrm{mg})$ Opioids between injury \& surgery Oral morphine equianalgesia (mg) Opioids peri-operative

Oral morphine equianalgesia (mg)

Types of opioid previously prescribed

Oxycodone

Hydrocodone

Hydromorphone

Codeine

Tramadol

Propoxyphene

\section{Trauma related factors}

Injury to dominant side

Carpal tunnel release during ORIF

\section{Second opioid prescription}

23\% (47)

$51 \pm 16$

$43 \%(20)$

$27 \pm 5.1$

$9.8 \%(4)$

$32 \%(15)$

$55 \%(26)$

$13 \%(6)$

$81 \%(38)$

$6.4 \%(3)$

$8.5 \%$ (4)

$4.3 \%(2)$

$13 \%(6)$

$2.5 \%$ (4)

0.011

$465 \pm 2501$

$34 \pm 261$

0.033

$47 \%(22)$

$43 \%(69)$

0.74

$184 \pm 276$

$123 \pm 196$

0.089

$77 \%(36)$

$82 \%(131)$

0.40

$393 \pm 304$

$377 \pm 256$

0.73

$78 \%$ (37)

$86 \%(137)$

0.25

36\% (17)

$4.3 \%(2)$

$4.3 \%(2)$

$26 \%(41)$

0.20

$3.8 \%(6)$

$3.8 \%(6)$

$1.9 \%(3)$

$1.9 \%$ (3)

1.0

1.0

0.32

$2.1 \%(1)$

$44 \%$ (19)

43\% (69)

0.74

$18 \%$ (29) 


\begin{tabular}{|c|c|c|c|}
\hline \multicolumn{4}{|l|}{ A0 classification } \\
\hline A & $43 \%(20)$ & $40 \%(65)$ & \multirow{3}{*}{0.21} \\
\hline B & $19 \%(9)$ & $11 \%(17)$ & \\
\hline C & $38 \%(18)$ & $49 \%(78)$ & \\
\hline Ulna fracture & $70 \%(33)$ & $54 \%(86)$ & 0.064 \\
\hline Days between injury and surgery & $8.5 \pm 5.7$ & $9.0 \pm 5.9$ & 0.61 \\
\hline \multicolumn{4}{|l|}{ Radiographic parameters after injury } \\
\hline Ulnarward inclination & $14 \pm 7.2$ & $11 \pm 8.7$ & 0.18 \\
\hline Ulnar variance & $1.9 \pm 5.1$ & $1.3 \pm 4.6$ & 0.52 \\
\hline Volar tilt & $-22 \pm 19$ & $-11 \pm 21$ & 0.0078 \\
\hline \multicolumn{4}{|c|}{ Radiographic parameters after surgery } \\
\hline Ulnarward inclination & $20 \pm 4.2$ & $20 \pm 4.7$ & 0.36 \\
\hline Ulnar variance & $0.17 \pm 3.2$ & $-0.32 \pm 2.9$ & 0.33 \\
\hline Volar tilt & $4.4 \pm 8.8$ & $6.3 \pm 8.0$ & 0.16 \\
\hline \multicolumn{4}{|l|}{ Questionnaires } \\
\hline Pain catastrophizing scale & $19 \pm 6.0$ & $18 \pm 7.1$ & 0.19 \\
\hline Numerical rating of pain intensity & $4.8 \pm 2.8$ & $3.3 \pm 2.4$ & $<0.001$ \\
\hline DASH score & $50 \pm 20$ & $47 \pm 18$ & 0.34 \\
\hline
\end{tabular}

$\mathrm{ASA}=$ American Society for Anesthesiologists; $\mathrm{ORIF}=$ open reduction and internal fixation; $\mathrm{DASH}=$ Disability of the Arm, Shoulder and Hand. Continuous variables as mean \pm standard deviation; discrete data as percentage (number). $P$ value $<0.05$ indicates statistically significant difference 
Appendix 3. Bivariate analysis of factors associated with disability and pain at suture removal after distal radius fracture surgery

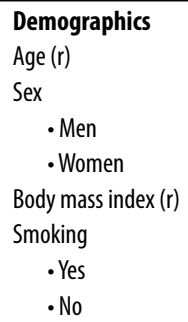

- No

\section{DASH score}

0.061

$45 \pm 18$

$49 \pm 19$

0.12

$57 \pm 18$

$47 \pm 18$

$44 \pm 18$

$49 \pm 19$

$56 \pm 17$

$49 \pm 18$

$44 \pm 20$

$35 \pm 12$

$55 \pm 23$

0.014

\section{$P$ value}

0.41

0.21

0.12

0.039

0.058

\section{Pain intensity \\ $-0.036$ \\ P value \\ 0.61}

$3.4 \pm 2.5$

$3.7 \pm 2.6$

0.031

$4.6 \pm 2.7$

$3.5 \pm 2.6$

$3.0 \pm 2.2$

$3.8 \pm 2.7$

$4.6 \pm 2.7$

$3.5 \pm 2.4$

$3.9 \pm 3.4$

$3.8 \pm 3.0$

$4.2 \pm 3.0$
0.43

0.67

0.097

0.047

0.69
$51 \pm 25$

$48 \pm 18$

0.011

$49 \pm 19$

$47 \pm 18$

0.12

$47 \pm 19$

$51 \pm 15$

$-0.098$

$47 \pm 18$

$56 \pm 19$

$45 \pm 19$

$49 \pm 18$

$56 \pm 14$

$48 \pm 19$

$45 \pm 22$

$48 \pm 19$

$52 \pm 26$

$48 \pm 18$

$68 \pm 7.1$

$48 \pm 19$
$4.4 \pm 3.1$

$3.6 \pm 2.5$

0.32

0.13

0.061

$3.7 \pm 2.6$

$3.5 \pm 2.6$

0.72

0.14

0.054

$3.6 \pm 2.6$

$3.7 \pm 2.4$

0.069

0.88

0.18

$3.6 \pm 2.6$

$3.9 \pm 2.7$

$3.9 \pm 2.8$

$3.5 \pm 2.5$

$3.6 \pm 1.8$

$3.6 \pm 2.6$

$2.8 \pm 1.2$

$3.6 \pm 2.6$

$5.0 \pm 2.6$

$3.6 \pm 2.6$

$6.5 \pm 2.6$

$3.5 \pm 2.5$

0.023 


\section{Trauma related factors}

Injury to dominant side

- Yes

- No

Carpal tunnel release during ORIF

- Yes

- No

A0 classification

-A

$\cdot B$

$\cdot C$

Ulna fracture

- Yes

- No

Days between injury and surgery $(r)$

Radiographic parameters after injury (r)

- Ulnarward inclination

- Ulnar variance

- Volar tilt

Radiographic parameters after surgery $(r)$

- Ulnarward inclination

- Ulnar variance

- Volar tilt

Psychological factor ( $r$ )

Pain Catastrophizing Scale
$55 \pm 19$

$43 \pm 17$

$<0.001$

$3.7 \pm 2.3$

$3.6 \pm 2.7$

$52 \pm 19$

$47 \pm 18$

0.13

$4.3 \pm 2.9$

$3.4 \pm 2.5$

$3.6 \pm 2.7$

$3.2 \pm 2.6$

$3.7 \pm 2.5$

$50 \pm 17$

$47 \pm 18$

0.80

$48 \pm 18$

$48 \pm 20$

$-0.0097$

$-0.084$

0.082

$-0.077$

$-0.11$

$-0.15$

$-0.044$

0.44

0.84

0.90

0.34

0.35

0.37

0.15

0.048

0.55

$<0.001$

0.31

$3.6 \pm 2.7$

$3.6 \pm 2.5$

0.058

$-0.031$

$-0.12$

$-0.12$

0.013

$-0.13$
0.98

0.42

0.71

0.16

0.15

$\begin{array}{ll}0.019 & 0.79\end{array}$

0.86

0.059

DASH = Disability of the Arm, Shoulder and Hand; ASA = American Society for Anesthesiologists; ORIF = open reduction and internal fixation. Continuous variables as mean \pm standard deviation; continuous data as Pearson correlation, indicated by $r$; $P$ value $<0.05$ indicates statistically significant difference. 
Discussion 


\section{This thesis addresses current issues in the outcome of operatively treated distal}

radius fractures. The general aim was to determine factors associated with adverse

events, motion, patient reported outcome measures, and opioid usage after surgery.

This thesis is divided in three main parts: (1) injury, (2) treatment, and (3) recovery.

\section{UNDERSTANDING THE INJURY}

Half of all distal radius fracture are intra-articular of which the majority (approximately $80 \%$ ) are complete articular, AO type C, fractures. ${ }^{1,2}$ Melone conceptually divided intra-articular fractures into a (1) radial styloid fragment, (2) volar lunate, and (3) dorsal lunate facet fragment. ${ }^{3}$ Medoff divided the intermediate column differently, recognizing a dorsal wall, ulnar corner, volar rim and free intra-articular fragments. ${ }^{4}$ These classifications are useful conceptually as they help characterize fracture patterns, defining important fracture elements, and directing fracture management. Yet, they are based on wisdom and merit confirmation. We used quantitative 3D computed tomography (Q3DCT) to measure fracture patterns and fragment characteristics such as displacement in 3 dimensions, articular surface area, and total area of the gap between fragments (see http://www.traumaplatform.org/currentprojects for a video of our methods).

We confirmed Melone's concepts about fracture patterns and determined that on average the volar lunate facet fragment is much larger than the dorsal lunate facet fragment and the radial styloid fragment had the greatest average displacement. Melone felt an unstable, malrotated, volar lunate facet fragment warranted open reduction and fixation. We found that in general volar lunate facet fragments are relatively large and least displaced, while dorsal lunate facet fragments are relatively small. This suggests that alignment of the volar lunate facet fragment with the radial styloid may be the key element of treatment and perhaps the dorsal lunate facet fragment may not routinely benefit from specific reduction and fixation.

Melone's concept encompasses predominantly complete intra-articular fractures. A more comprehensive division is the systematic AO/OTA classification. ${ }^{2,5,6}$ This classification is commonly used today and is recommended by the Dutch distal radius fracture guidelines. ${ }^{7}$ The AO/OTA classification mainly facilitates international, scientific, communication. A large, international cohort might best assess its observer reliability. In a cohort of 65 observers, rating 96 images, we found that there was less agreement when classifying consensus type B fractures compared to type A or C fractures. Also, there was a difference in reliability between groups from different countries when classifying type B fractures. Scientific communication on type $B$ fractures might benefit from further classification information and consideration, for instance by a group of surgeons reaching consensus, rather than a single observer. We found no difference in reliability between residents and surgeons, or between specialties. This suggests the AO/OTA classification can relatively easily be learned and does not depend on experience. It may even be possible to program a computer to analyze CT scans and classify fractures, which might increase accuracy and reliability.

There are myriad one-dimensional radiographic and CT measures to quantify distal radius fracture alignment. Most of those measures correlate poorly with patient reported outcomes. ${ }^{8,9}$ In chapter 5 we determined that step-off (a measure often recommended to decide for operative treatment) has a particularly low inter-observer reliability. Also, radiographic and CT step-off and gap measurements were poorly correlated. Q3DCT can 
however more reliably determine detailed fracture characteristics. This method could aid in choosing the right treatment of complete articular distal radius fractures and in other joints, especially in areas of debate. By applying this technique in chapter 6 we assessed if the newly developed Q3DCT measures of 3D displacement and gap surface area were better associated with range of motion and patient reported outcomes than one-dimensional measures.

\section{TREATMENT}

Patients with a distal radius fracture are increasingly offered surgery to prevent healing with deformity ${ }^{10}$. This is often the case in patients with shearing fractures of the articular margin, fracture-dislocation, and fractures with extensive fragmentation of the articular surface and the metaphysis/diaphysis in particular. ${ }^{11}$ When surgery is planned before reduction, we typically still reduce the fracture prior to surgery because we assume this reduces soft tissue tension and pressure on the median nerve. However, the risk of soft tissue problems, median neuropathy related to deformity, and increased pain is debatable. In a cohort of 1511 patients, including 102 whose fractures were not reduced prior to surgery, we found no difference in adverse events or reoperations between reduced and unreduced fractures. For patients who choose operative treatment prior to reduction and have no nerve or skin issues, manipulative reduction may thus not be helpful. Before implementing these findings, our results would benefit from prospective conformation. It would also be interesting to assess differences in symptoms and disability, overall satisfaction and pain levels. However, based on our study, patients and surgeons can safely consider foregoing the recommendation made by the Dutch distal radius fracture guidelines to reduce any displaced fracture. ${ }^{7}$ The decision to proceed with surgery and forgo reduction has to be made together with the patient. Decision aids - tools to facilitate the shared decision-making process, i.e., increasing patient participation - can provide balanced, accessible, and dispassionate information about the harms and benefits of surgery, and aid patients and doctors in their decision. A freely available decision aid for displaced distal radius fractures was recently developed in English ${ }^{12}$ and Dutch ${ }^{13}$ (www.decionaid.info \& www.keuzehulp.info). In other areas these tools increased patient involvement and decreased their uncertainty about which course of action to take (i.e., decisional conflict). Patients were also more likely to select the least invasive treatment. ${ }^{14}$ The effect of a decision aid remains to be confirmed in the treatment of displaced distal radius fractures, but increased influence of patient preferences reflects optimal respect for patients.

When a patient chooses surgery, the goal is usually to improve alignment. But it's unknown to what extent reduction is maintained by a volar locking plate. Comparing postoperative radiographic and CT measures with the position one year after surgery, we found a small (less than 2 millimeters or 2 degrees) but statistically significant change in several measures. Accounting for inter-observer agreement, this is probably within measurement error. Additionally, one patient had more than $3^{\circ}$ loss of palmar tilt, and one patient experienced an increase in gap more than $3 \mathrm{~mm}$, one patient worsened more than $3 \mathrm{~mm}$ on each CT measure, except for frontal step off where no patient worsened more than $3 \mathrm{~mm}$. After fixation with a variable angle locking plate minimal fracture settling is expected on average.

We also found no difference in change in fracture position or range of motion, grip strength or PRWE between one and two distal screw rows. This confirms previous studies. ${ }^{15,16}$ Routinely using two rows of screws seems to add costs, a longer duration of surgery, and more opportunities for a misplaced or overly long screw. 
Many surgeons and patients follow the traditional biomedical model of illness and assume a direct correspondence between nociception (e.g., magnitude of displacement) and pain (the experience of discomfort). Yet, the correlation between deformity in distal radius fracture and patient reported outcomes (e.g., DASH or PRWE scores) is limited. ${ }^{9}$ In a cohort of 59 limited displaced fractures, we found that some radiographic measures were associated with change in range of motion, grip strength, PRWE and EQ5D at 12 weeks, but no measure was associated with symptoms or objective impairment one year after fracture. The fact that some residual displacement is not associated with impairment or patient reported outcome should be considered when counseling patients on the risks and benefits of surgical treatment. Also, since no radiographic variables were associated with impairment or patient reported outcome at one year, the prognostic value of Q3DCT measures is limited in distal radius fractures.

The variation seen in recovery between patients might be related to other factors such as the tendency to misinterpret or overinterpret nociception (i.e., catastrophic thinking), heightened concern about illness, and social and cultural factors on illness behavior. ${ }^{17}$ In chapter 9, we found no radiographic factors associated with QuickDASH at suture removal. Greater dorsal angulation after surgery was associated with greater pain intensity at suture removal, but only explained $1.4 \%$ of the variation. Catastrophic thinking, a measure of a less effective coping strategy, was more strongly associated with both QuickDASH and pain intensity.

\section{ASPECTS OF RECOVERY}

The biopsychosocial model that accounts for thoughts, emotions, and behaviors that accompany illness might be more appropriate than the traditional biomedical model that tries to reduce all symptoms and limitations to pathophysiology. This paradigm accounts for the influence of psychological factors in determining pain intensity and magnitude of limitations ${ }^{18,19}$ and the limited correlation between nociception and patient reported outcomes (as found in chapter 6). We found that catastrophic thinking was a consistent and major determinant of finger stiffness at suture removal and six weeks after injury. The correlation of a normal human response to nociception with finger stiffness makes it less likely that we will identify an, as yet elusive, unmeasured pathophysiology. Instead finger stiffness occurs due to normal human illness behavior, namely the tendency to misinterpret or overinterpret nociception (catastrophic thinking) and subsequent fear and avoidance of activity. This causes stiffness and skin changes (swelling, shiny skin, changes in hair patterns) associated with disuse. ${ }^{20}$ Labels such as complex regional pain syndrome and reflex sympathetic dystrophy may be social constructions: entities that exist only because a society behaves as if they exist - as opposed to diseases like influenza and lung cancer. Labeling patients with these illness constructions is probably unhelpful. Instead we should focus on helping patients manage their catastrophic thinking. Future research should explore if this facilitates recovery after distal radius fractures too - a strategy that showed to be successful in patients with lower back pain. ${ }^{21,22}$

Interventions targeting psychological measures have been proposed to improve post-surgical outcomes in total knee, hip and shoulder arthorplasty. ${ }^{23-26}$ If we are to implement this in trauma, we need to know how psychological constructs behave during recovery. We found that symptoms of depression and catastrophic thinking, but not health anxiety, improved during recovery after upper extremity injury. If we want to improve outcome by targeting catastrophic thinking and depression, or use these measures to predict outcome, we need to account for the phase of recovery. Future research can assess if threshold values can be determined at which patients are at risk for a slow recovery due to a higher tendency to overinterpret nociception. 
Over the last two decades the United States moved to an opioid centric pain model, creating an epidemic of prescription opioid abuse, without increasing satisfaction with pain relief. ${ }^{27-29}$ We studied factors associated with a second opioid prescription after distal radius fracture surgery, hoping we would find amendable causes that could inform better pain management protocols and encourage decreased and safer use of opioids. We found male sex was associated with greater opioid consumption. This should make doctors more apprehensive about the amount of opioids prescribed to men. Greater dorsal angulation was the only factor reflecting injury severity associated with greater opioid consumption, but explained only $3.3 \%$ of the variation. Awareness of the limited association between pathophysiology and opioid consumption might make doctors more aware of other causes increasing opioid use, such as patients' expectations prior to surgery (in particular the realization that it's normal to have pain after injury and surgery) and cultural factors. ${ }^{28,30}$ Although the clinic this study was conducted, was quite strict with opioids (20 5mg oxycodone with acetaminophen pills after surgery) compared to American standards, in the Netherlands we use far fewer opioids. Yet, after ankle fracture surgery, pain relief and satisfaction are no different between the United States and the Netherlands. ${ }^{28}$ The fact that fewer opioids can achieve similar pain relief and satisfaction should deter countries, like the Netherlands, with actually strict opioid policies moving towards an opioid centric pain model.

\section{CONCLUSIONS AND FUTURE PERSPECTIVES}

For patients who make an informed decision to undergo operative treatment for their closed, neurovascular intact, displaced distal radius fracture - no matter what manipulative reduction might achieve - leaving the fracture unreduced is safe. This warrants a less strict recommendation for reduction by the Dutch distal radius fracture guidelines. But our results also need prospective validation and future studies might address overall pain and satisfaction.

We may never be able to formulate evidence-based criteria for operative treatment of displaced distal radius fractures. Limited displacement seems not to greatly affect short-term (chapters 6,7 and 9) or long-term (chapter 6) patient reported outcomes. Any dichotomous cut-off is clinically impractical by definition. For example, considering a step-off of more than $2 \mathrm{~mm}$, recommended by the AAOS distal radius fracture guidelines ${ }^{31}$ : the difference between $1.9 \mathrm{~mm}$ and $2.1 \mathrm{~mm}$ lies within what's expected due to measurement error (chapter 5). This is something that's important to discuss with our patients, potentially aided by shared decision making tools. The greatest potential for functional improvement after distal radius fracture seems to lie in addressing coping strategies. Recent research showed that simple coaching after radial head fractures directly increased elbow range of motion. ${ }^{32}$ This is something that starts at the first clinic visit by showing empathy and understanding. Let the patient tell their story. Research shows it's not the duration of the interaction, but the quality that counts. ${ }^{33,34}$ It might be helpful to train doctors in recognizing and dealing with signs and symptoms of catastrophic thinking, e.g., patients using words such as "unbearable" or "excruciating", or they carry their hand as if it was detached. ${ }^{35}$ The development, coaching, and training of strategies addressing catastrophic thinking and their effect should be part of future research; not the movement towards an opioid centric pain management model.

Recently the Distal Radius Fracture Outcome Consortium published a summary checklist of minimum outcomes to capture after distal radius fracture in order to establish a unified approach to outcomes assessment. ${ }^{36}$ Measuring coping strategies is not part of this list. Yet, it might be one of the most important factors determining range of motion and patient reported outcome after surgery. ${ }^{37}$ 


\section{CAN COMPLETE ARTICULAR FRACTURES BE DIVIDED ACCORDING TO MELONE'S CONCEPT?}

We confirmed Melone's concepts about fracture patterns. We found that volar lunate fragments are relatively large and least displaced, while dorsal lunate fragments are relatively small. This suggests that alignment of the volar lunate fragment with the radial styloid may be the key element of treatment and perhaps the dorsal lunate fragment may not routinely benefit from specific reduction and fixation.

\section{CAN Q3DCT BE USED TO ACCURATELY ASSESS INTRA-ARTICULAR DISTAL RADIUS FRACTURE CONFIGURATION AND DISPLACEMENT?}

Q3DCT measures are highly accurate. The intraclass correlation coefficients were:

- 3D displacement: $0.82(95 \% \mathrm{Cl} 0.69$ to 0.90$)$

- articular surface area: $0.91(95 \% \mathrm{Cl} 0.84$ to 0.96$)$

- gap surface area $0.93(95 \% \mathrm{Cl} 0.74$ to 0.98$)$

\section{IS THE INTER-OBSERVER RELIABILITY SIMILAR FOR AO TYPE A, B AND C FRACTURES?}

AO/OTA type B fractures were least reliably classified, followed by type $C$ and type $A$ fractures. The intraclass correlation coefficients were:

- Type A (substantial): $0.68(95 \% \mathrm{Cl} 0.62$ to 0.74$)$.

- Type B (fair agreement): 0.28 (95\% Cl 0.23 to 0.35$)$

- Type C (moderate agreement): $0.44(95 \% \mathrm{Cl} 0.37$ to 0.52$)$

Scientific communication on type B fractures might benefit from further classification information, for example a group of surgeons reaching consensus, rather than a single observer.

\section{IS CLOSED REDUCTION WORTHWHILE FOR THE SUBSET OF PATIENTS WHO CHOOSE OPERATIVE TREATMENT PRIOR TO ATTEMPTED REDUCTION OF THEIR DISTAL RADIUS FRACTURE?}

We found no difference in complications and reoperations between reduced and unreduced fractures prior to surgery. Patients who make an informed decision to undergo operative treatment for their closed, neurovascular intact displaced distal radius fracture - no matter what manipulative reduction might achieve - reduction may not be helpful.

\section{HOW WELL IS ALIGNMENT MAINTAINED ONE YEAR AFTER TREATMENT WITH A VOLAR LOCKING PLATE ASSESSED BY RADIOGRAPHS AND CT SCANS?}

We found a small (less than $2 \mathrm{~mm}$ or 2 degrees) but statistically significant change in several measures. Accounting for inter-observer agreement, this is probably within measurement error. Only minimal change in reduction can be expected after volar plate fixation. 
6 IS THERE A DIFFERENCE IN FRACTURE POSITION OR FUNCTIONAL OUTCOME BETWEEN ONE OR TWO DISTAL SCREW ROWS?

We found no difference in change in fracture position between one and two distal screw rows. There was also no difference in functional outcome at one year. Using only one row of distal screws might lower the cost of surgery, reduce operation time, and limit opportunities for a misplaced or overly long screw.

\section{ARE RADIOGRAPHIC, CONVENTIONAL CT AND Q3DCT MEASURES ASSOCIATED WITH} CHANGE IN PATIENT REPORTED AND OBJECTIVE OUTCOMES ONE YEAR AFTER TREATMENT WITH A VOLAR LOCKING PLATE?

Some radiographic and Q3DCT measures are associated with early recovery, but no measure is associated with symptoms or objective impairment at one year after fracture. This should be considered when counseling patients on the risks and benefits of surgical treatment, especially when gross malalignment is absent and in older, low demand patients. The prognostic value of Q3DCT measures is limited in distal radius fractures.

8 WHAT FACTORS ARE ASSOCIATED WITH FINGER STIFFNESS AT SUTURE REMOVAL AFTER VOLAR LOCKING PLATE FIXATION, SPECIFICALLY CATASTROPHIC THINKING (NEGATIVE BELIEFS ABOUT PAIN LEADING TO AN OVERPROTECTIVE RESPONSE)?

Catastrophic thinking was a consistent and major determinant of finger stiffness at suture removal and six weeks after injury. Future research should assess if treatments that ameliorate catastrophic thinking can facilitate recovery of finger motion after operative treatment of a distal radius fracture.

9 IS THERE A DIFFERENCE IN SYMPTOMS OF (1) DEPRESSION, (2) HEALTH ANXIETY, AND (3) CATASTROPHIC THINKING DURING THE RECOVERY AFTER RADIUS FRACTURE? Symptoms of depression and catastrophic thinking, but not health anxiety, improved during recovery after injury. If psychological measures are used as a screening tool to predict outcome after treatment, one should account for a patient's disease phase.

\section{ARE THERE ANY DIFFERENCES BETWEEN PATIENTS WHO DO AND DO NOT RECEIVE} A SECOND OPIOID PRESCRIPTION FOLLOWING TREATMENT OF THEIR DISTAL RADIUS FRACTURE WITH A VOLAR LOCKING PLATE?

One measure of fracture severity (dorsal displacement) was independently associated with a second opioid prescription, but alone it accounted for $3.3 \%$ of the variation. Other factors such as the patient's expectation prior to surgery, in particular the realization that injury and surgery hurt, might be addressed in future research. 


\section{REFERENCES}

1. McQueen MM, Jupiter JB. Radius and ulna. Butterworth-Heinemann; 1999.

2. Müller ME. The Comprehensive classifiction of fractures of long bones. Berlin ;New York: Springer-Verlag; 1990.

3. Melone CP, Jr. Distal radius fractures: patterns of articular fragmentation. Orthop Clin North Am. 1993;24(2):239-253.

4. Medoff RJ. Essential radiographic evaluation for distal radius fractures. Hand Clin. 2005;21 (3):279-288.

5. Marsh JL, Slongo TF, Agel J, et al. Fracture and dislocation classification compendium - 2007: Orthopaedic Trauma Association classification, database and outcomes committee. J Orthop Trauma. 2007;21(10 Suppl):S1-133.

6. Müller ME, Nazarian S, Koch P. Classification AO des fractures: les os longs. Berlin: Springer-Verlag; 1987.

7. Nederlandse Vereniging voor Heelkunde. Richtlijn distale radius fracturen: diagnostiek en behandeling. 2010.

8. Forward DP, Davis TR, Sithole JS. Do young patients with malunited fractures of the distal radius inevitably develop symptomatic post-traumatic osteoarthritis? J Bone Joint Surg Br. 2008;90(5):629-637.

9. Souer JS, Lozano-Calderon SA, Ring D. Predictors of wrist function and health status after operative treatment of fractures of the distal radius. J Hand Surg Am. 2008;33(2):157-163.

10. Chung KC, Shauver MJ, Birkmeyer JD. Trends in the United States in the treatment of distal radial fractures in the elderly. J Bone Joint Surg Am. 2009;91(8):1868-1873.

11. Mackenney PJ, McQueen MM, Elton R. Prediction of instability in distal radial fractures. J Bone Joint Surg Am. 2006;88(9):1944-1951.

12. Teunis T, Mulders MAM, Fischerauer SF, Goslings JC, Ring D. Displaced distal radius fracture. 2016; http://www.decisionaid.info/pp/distalradius/intro. Accessed 04-14-2016.

13. Mulders MAM, Teunis T, Fischerauer SF, Ring D, Goslings JC. Verplaatste gebroken pols. 2016; https://www.keuzehulp.info/pp/gebrokenpols/intro. Accessed 04-14-2016.

14. Stacey D, Legare F, Col NF, et al. Decision aids for people facing health treatment or screening decisions. Cochrane Database Syst Rev. 2014;1:CD001431.

15. Kawasaki K, Nemoto T, Inagaki K, Tomita K, Ueno Y. Variable-angle locking plate with or without double-tiered subchondral support procedure in the treatment of intra-articular distal radius fracture. J Orthop Traumatol. 2014;15(4):271-274.

16. Neuhaus V, Badri O, Ferree S, Bot AG, Ring DC, Mudgal CS. Radiographic alignment of unstable distal radius fractures fixed with 1 or 2 rows of screws in volar locking plates. J Hand Surg Am. 2013;38(2):297-301.

17. Teunis T, Bot AG, Thornton ER, Ring D. Catastrophic Thinking is Associated with Finger Stiffness After Distal Radius Fracture Surgery. J Orthop Trauma. 2015.

18. Vranceanu AM, Bachoura A, Weening A, Vrahas M, Smith RM, Ring D. Psychological factors predict disability and pain intensity after skeletal trauma. J Bone Joint Surg Am. 2014;96(3):e20.

19. Vranceanu AM, Barsky A, Ring D. Psychosocial aspects of disabling musculoskeletal pain. J Bone Joint Surg Am. 2009;91(8):2014-2018.

20. Linton SJ, Shaw WS. Impact of psychological factors in the experience of pain. Phys Ther. 2011;91(5):700-711.

21. Wertli MM, Burgstaller JM, Weiser S, Steurer J, Kofmehl R, Held U. Influence of catastrophizing on treatment outcome in patients with nonspecific low back pain: a systematic review. Spine (Phila Pa 1976). 2014;39(3):263-273.

22. Wertli MM, Eugster R, Held U, Steurer J, Kofmehl R, Weiser S. Catastrophizing-a prognostic factor for outcome in patients with low back pain: a systematic review. Spine J. 2014;14(1 1):2639-2657.

23. Sullivan M, Tanzer M, Stanish W, et al. Psychological determinants of problematic outcomes following Total Knee Arthroplasty. Pain. 2009;143(1-2):123-129.

24. Wong SE, Zhang AL, Berliner JL, Ma CB, Feeley BT. Preoperative patient-reported scores can predict postoperative outcomes after shoulder arthroplasty. J Shoulder Elbow Surg. 2016.

25. Berliner JL, Brodke DJ, Chan V, SooHoo NF, Bozic KJ. Can Preoperative Patient-reported Outcome Measures Be Used to Predict Meaningful Improvement in Function After TKA? Clin Orthop Relat Res. 2016.

26. Berliner JL, Brodke DJ, Chan V, SooHoo NF, Bozic KJ. John Charnley Award: Preoperative Patient-reported Outcome Measures Predict Clinically Meaningful Improvement in Function After THA. Clin Orthop Relat Res. 2016;474(2):321-329.

27. Bot AG, Bekkers S, Arnstein PM, Smith RM, Ring D. Opioid use after fracture surgery correlates with pain intensity and satisfaction with pain relief. Clin Orthop Relat Res. 2014;472(8):2542-2549.

28. Helmerhorst GT, Lindenhovius AL, Vrahas M, Ring D, Kloen P. Satisfaction with pain relief after operative treatment of an ankle fracture. Injury. 2012;43(11):1958-1961.

29. Helmerhorst GT, Vranceanu AM, Vrahas M, Smith M, Ring D. Risk factors for continued opioid use one to two months after surgery for musculoskeletal trauma. J Bone Joint Surg Am. 2014;96(6):495-499. 
30. Carragee EJ, Vittum D, Truong TP, Burton D. Pain control and cultural norms and expectations after closed femoral shaft fractures. Am J Orthop (Belle Mead NJ). 1999;28(2):97-102.

31. American Academy of Orthopaedic Surgeons. The treatment of distal radius fractures: guidelines and evidence report. 2009.

32. Teunis T, Thomson R, Guitton TG, Vranceanu AM, Ring D. Coaching of patients with an isolated minimally displaced fracture of the radial head immediately increases range of motion. J Hand Ther. 2016;in print.

33. Teunis T, Thornton ER, Jayakumar P, Ring D. Time Seeing a Hand Surgeon Is Not Associated With Patient Satisfaction. Clin Orthop Relat Res. 2015;473(7):2362-2368.

34. Menendez ME, Chen NC, Mudgal CS, Jupiter JB, Ring D. Physician Empathy as a Driver of Hand Surgery Patient Satisfaction. J Hand Surg Am. 2015;40(9):1860-1865 e1862.

35. Bot AG, Vranceanu AM, Herndon JH, Ring DC. Correspondence of patient word choice with psychologic factors in patients with upper extremity illness. Clin Orthop Relat Res. 2012;470(11):3180-3186.

36. Waljee JF, Ladd A, MacDermid JC, Rozental TD, Wolfe SW, Distal Radius Outcomes C. A Unified Approach to Outcomes Assessment for Distal Radius Fractures. J Hand Surg Am. 2016.

37. Teunis T, Ring D. Comprehensive Outcome Assessment After Distal Radius Fracture. J Hand Surg Am. 2016. 
Summary 
The forearm has two bones: the radius and the ulna. The end of the radius and ulna form one side of the wrist joint. Fractures of the radius at the wrist are called "distal radius fractures" (Figure 1) and are one of the most common fractures. Fractures where the bones are far out of place may heal with a bend. If the bend is limited, or for patients who prefer deformity to surgery, the fracture can be treated without surgery. If there is substantial deformity, and the patient chooses surgery, the fracture can be treated with an operation. The aim of this thesis was to determine factors associated with adverse events, loss of motion, functional limitations, and opioid use after surgery. This work is divided in three parts: (1) injury, (2) treatment, and (3) recovery.

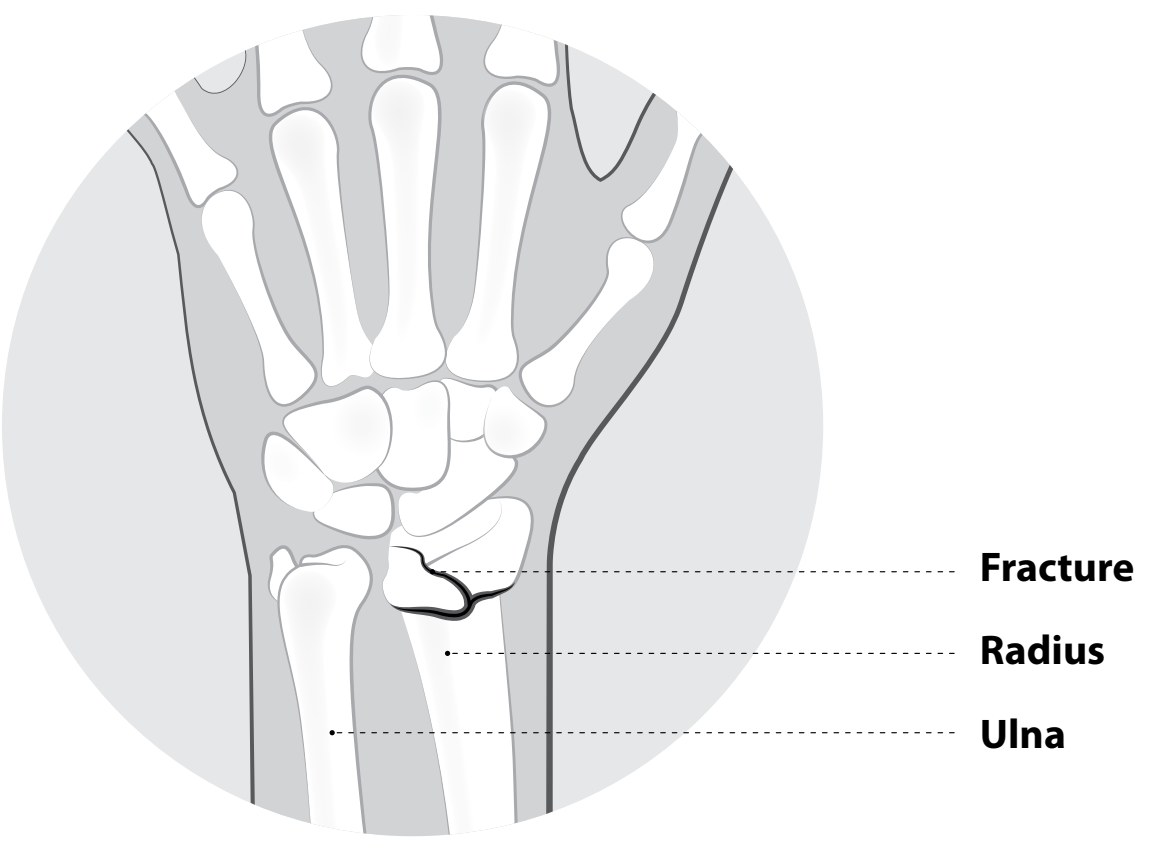

FIGURE 1. THE TWO BONES IN THE FOREARM: THE RADIUS AND THE ULNA; THE FRACTURE AT THE WRIST IS CALLED A DISTAL RADIUS FRACTURE. 


\section{PART 1: UNDERSTANDING THE INJURY}

About half of all distal radius fractures involve the joint (where the bones move against one another). Joint fractures often consist of three major fragments: (1) radial styloid fragment, (2) volar lunate facet, and (3) dorsal lunate facet fragment (Figure 2).

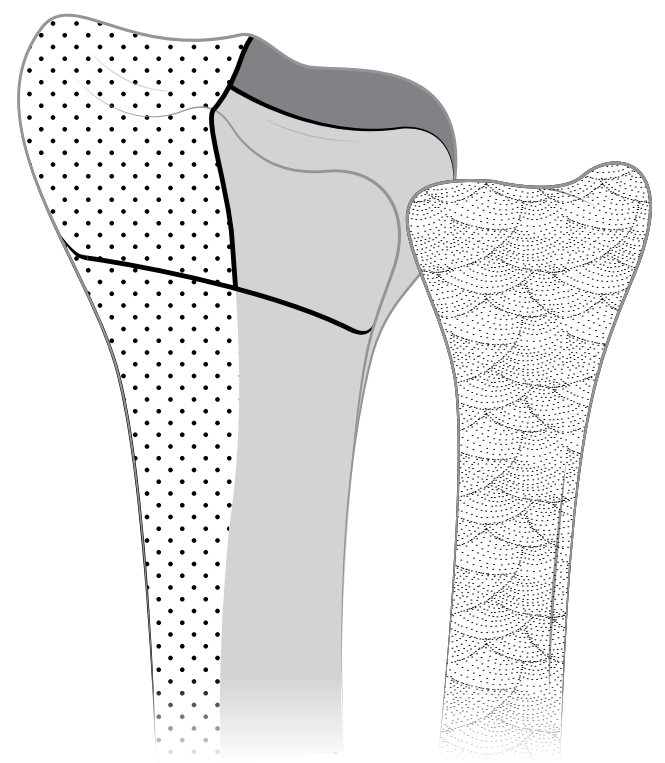

\section{Fracture fragments}

- Potential fracture lines

$\because$ Radial styloid fragment

Dorsal lunate facet fragment

Volar lunate facet fragment

\section{FIGURE 2. BASIC COMPONENTS INVOLVED IN A TYPICAL DISTAL RADIUS JOINT FRACTURE.}

The goal of surgery is to align the joint surface. If some fragments are more important to realign than others, we should focus on these fragments during surgery. We created fifty 3D models based on CT scans of intraarticular distal radius fractures (also referred to as quantitative 3D computed tomography or Q3DCT). We measured fracture patterns and fragment characteristics such as displacement in three dimensions, articular surface area, and the total area of the gap between fragments. We found that on average the volar lunate facet fragment is the largest. The radial styloid fragment is in general the most out of place. This suggests that alignment of the volar lunate facet fragment with the radial styloid fragment may be most important. Perhaps it is not necessary to make a separate incision to fix the dorsal lunate facet fragment. The AO/OTA fracture classification classifies all different kinds of distal radius fractures systematically. It has three main groups: (A) fractures outside of the joint, (B) fractures separating part of the joint from the shaft, and $(C)$ fractures into the joint that completely separate the joint surface from the shaft. This classification helps researchers communicate about fracture types. We tested how reliable this classification was. An international cohort of 65 doctors classified 96 different distal radius fractures. We found that there was less agreement when classifying consensus type B fractures compared to type $A$ or $C$ fractures. Also, there was a difference in reliability between groups from different countries when classifying type $B$ fractures. When researches study type B fractures they need to carefully consider and report how they are classifying the fractures. 


\section{PART 2: TREATMENT}

When patients have a fracture that is out of place, the fracture is usually realigned in the Emergency Department. If the fracture can't be realigned properly, or there is a high chance of losing alignment, patients can choose surgery to avoid deformity (Figure 3).

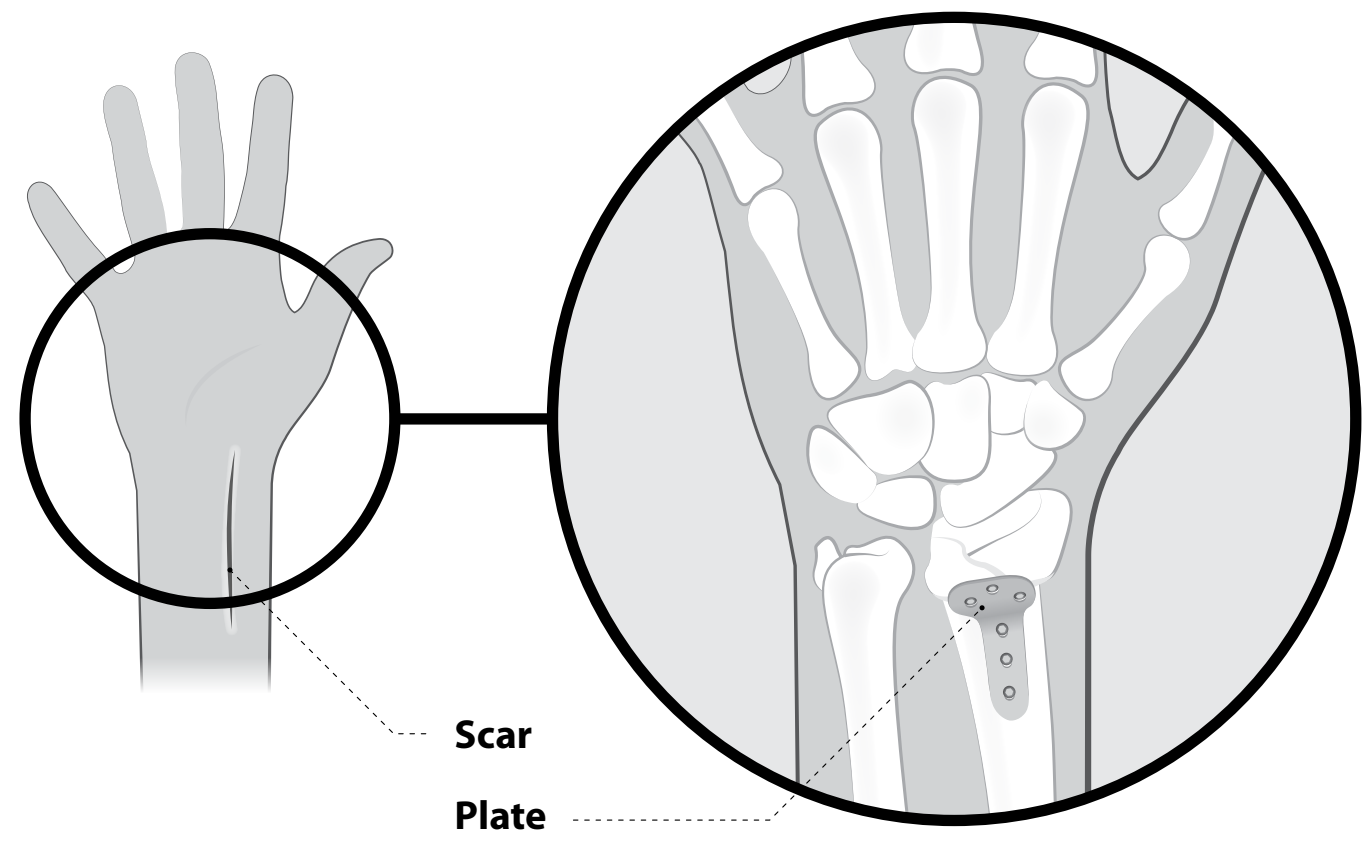

FIGURE 3. THE DISTAL RADIUS FRACTURE IS FIXED WITH A PLATE.

To help patients and surgeons decide on the best treatment we developed a decision aid (www.decisionaid. info/pp/distalradius). Sometimes, depending on the fracture type, the patient and the surgeon choose surgery no matter what reduction might achieve. We often still reduce the fracture because we assume this reduces skin tension, or pressure on the nerves. But we don't know if this is worthwhile. Reduction itself is also painful. We studied if not reducing the fracture before surgery results in more adverse events and more surgery. We compared 102 patients with an unreduced fracture to 1409 people with a reduced fracture before surgery. We found no difference between the two groups in terms of adverse events or number of additional surgeries. For patients who choose surgery before reduction, and who have no nerve or skin issues, it is safe to forego reduction.

We also assessed if the fracture fragments stay in place after surgery to apply a locked volar plate. After 1 year we found a change in several measurements on radiographs and CT scans. But the changes were small: in general less than 2 millimeters or degrees. Only one patient changed more than 3 millimeters or degrees in several measures. These changes are so small they might be due to measurement inaccuracy or unreliability. After surgery with a plate fractures mostly stay in place. In the same study we also assessed if two rows of screws are better than one row. We found no difference in motion, grip strength, or limitations. It's cheaper, faster and safer to use only one row of screws. If you use more screws it takes more time and you have a higher change of placing them incorrectly. 
"The further the broken bones remain out of place, the less well the arm will function", or so many doctors and patients assume. This fits the biomedical model where what you feel and how the body is affected is directly related to the severity of the disease or injury. To test this, we assessed if residual displacement was associated with symptoms and limitations, range of motion and grip strength one year after surgery with a locked volar plate. We have to keep in mind that patients in this study all had surgery, so the fracture fragments were not far out of place. Yet, no matter how we measured it, residual displacement was not associated with greater symptoms and limitations, or less motion or grip strength at one year. There seem to be other factors that determine the result after surgery.

\section{PART 3: ASPECTS OF RECOVERY}

The biopsychosocial model also accounts for thoughts, emotions, and behaviors. This model might be better at determining the results after surgery of distal radius fractures than the biomedical model. We found that 6 weeks after injury catastrophic thinking - the tendency to prepare for the worse and feel protective when in pain - was the most consistent factor determining finger stiffness. Catastrophic thinking causes finger stiffness and skin changes (swelling, shiny skin, changed in hair patterns) associated with disuse. Labels such as complex regional pain syndrome may medicalize this normal human illness behavior in response to injury. We could research if helping people manage their catastrophic thinking can enhance their recovery.

When looking more closely at psychological measures we discovered that symptoms of depression and catastrophic thinking (but not health anxiety) improve during recovery. When using these measures we need to account for the phase of recovery patients are in.

People in the United States and Canada consume most of the world's opioids. These opioids don't seem to increase their satisfaction with pain relief. We studied what factors were associated with requesting more opioids after distal radius fracture surgery. We found that men and more angled fractures had a significant, but very small association with greater opioid intake. Work to date established that people in the Netherlands take less opioids for ankle fractures than people in the United States, but their pain intensity and satisfaction with pain relief are no different; that opioid use 1 month after injury is associated with depression and posttraumatic stress; and that greater opioid intake is associated with more pain and less satisfaction with pain relief. Psychosocial factors (stress, distress, and less effective coping strategies) seem to be the key to effective pain relief. 


\section{CONCLUSIONS}

1. In distal radius fracture surgery alignment of the volar lunate facet fragment with the radial styloid fragment may be most important. Perhaps it is not necessary to make a separate incision to fix the dorsal lunate facet fragment.

2. Type B fractures are harder to classify. When researches write papers on type B fractures they need make sure they need to carefully consider and report how they are classifying the fractures.

3. Patients can use a decision aid to help them choose between surgery or manipulation and immobilization. For patients who choose surgery before reduction, and who have no nerve or skin issues, it is safe to forgo reduction.

4. After volar locking plate fixation, fracture fragments mostly stay in place.

5. One row of screws seems to be as good as two rows.

6. How far the fracture fragments remain out of place after surgery doesn't affect symptoms and limitations, motion, or grip strength at 1 year.

7. Greater catastrophic thinking causes greater finger stiffness after surgery.

8. Symptoms of depression and catastrophic thinking, but not health anxiety, improve during recovery after injury.

9. How far the fracture fragments are apart only has a limited effect on the amount of opioids people take. 
Summary in Dutch

Samenvatting in het Nederlands 
De onderarm bestaat uit twee botten: de radius (spaakbeen) en de ulna (ellepijp). De uiteinden van de radius en ulna vormen samen één kant van het polsgewricht. Als het uiteinde van de radius bij de pols gebroken is, noemen we dit een "distale radius fractuur" (Figuur 1). Dit is een van de meest voorkomende breuken. Bij sommige breuken zijn de botdelen verplaatst. Deze breuken genezen met een knik. Als de knik klein is, of als patiënten liever een knik hebben dan een operatie, kan de breuk met gips worden behandeld. Als de botdelen ver uitelkaar staan, en de patiënt kiest voor een operatie, dan kan de breuk worden geopereerd. Het doel van dit proefschrift is vast te stellen welke factoren geassocieerd zijn met complicaties, verminderde beweging, functiebeperkingen, en opiaatgebruik na een operatie. Dit werk is opgedeeld in drie delen: (1) traumamechanisme, (2) behandeling, (3) herstel.

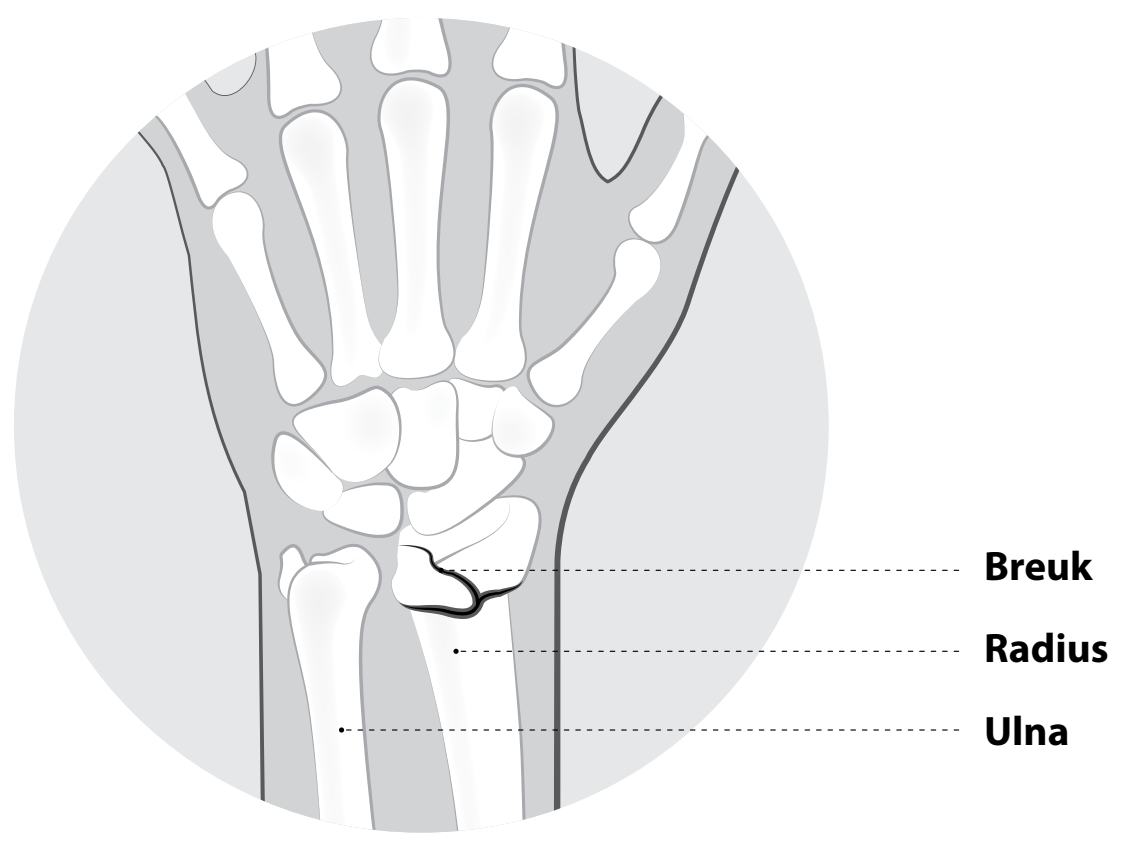

FIGUUR 1. DE TWEE BOTTEN IN DE ONDERARM: DE RADIUS EN DE ULNA; DE BREUK BIJ DE POLS HEET EEN DISTALE RADIUS FRACTUUR. 


\section{DEEL 1: TRAUMAMECHANISME}

Ongeveer de helft van alle distale radius fracturen loopt door tot in het polsgewricht. Breuken tot in het gewricht bestaan meestal uit drie fragmenten: (1) styloideus radii fragment, (2) volaire fossa lunatum fragment, en (3) dorsale fossa lunatum fragment (Figuur 2).

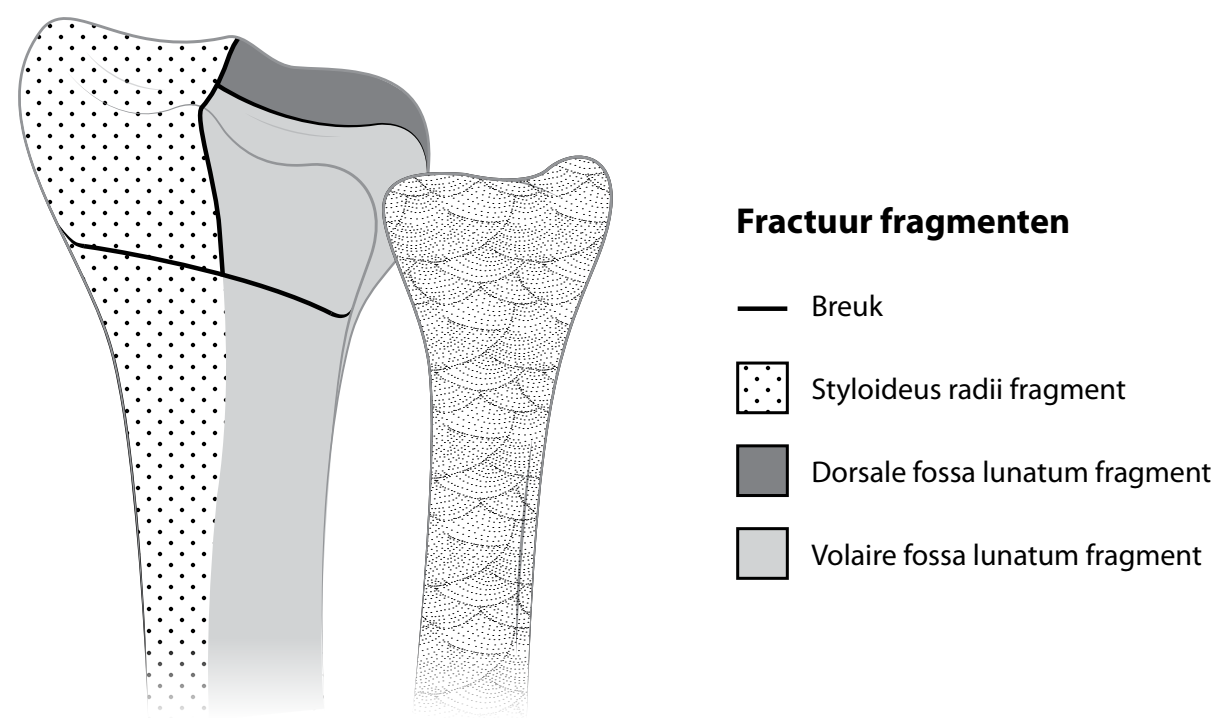

\section{FIGUUR 2. DE DRIE TYPISCHE FRAGMENTEN IN EEN DISTALE RADIUS FRACTUUR DIE TOT IN HET GEWRICHT LOOPT.}

Als de botdelen bij het gewricht verplaatst zijn, kan dit mogelijk klachten geven. Het doel van een operatie is om deze fragmenten weer op een lijn te plaatsen. Als sommige fragmenten belangrijker zijn om uit te lijnen dan andere, moeten we onze aandacht op die belangrijke fragmenten richten tijdens een operatie. We hebben 3D modellen gemaakt van 50 distale radius fracturen die tot in het gewricht lopen. Deze methode wordt "quantitative 3D computed tomography" of Q3DCT genoemd. Vervolgens hebben we het fractuurpatroon, de verplaatsing en de grootte van de fragmenten en de grootte van het gat tussen de fragmenten gemeten. We vonden dat gemiddeld het volaire fossa lunatum fragment het grootst is. Het styloideus radii fragment is gemiddeld het meest verplaatst. Dit suggereert dat het uitlijnen van het volaire fossa lunatum fragment met het styloideus radii fragment het belangrijkste onderdeel van de operatie is. Misschien is het dus niet nodig om een aparte incisie te maken om ook het dorsale fossa lunatum fragment recht te zetten.

De AO/OTA fractuur classificatie deelt alle verschillende distale radius fracturen systematisch in. De classificatie bestaat uit drie groepen: (A) breuken buiten het gewricht, (B) breuken die een gedeelte van het gewricht scheiden van de schacht, (C) breuken tot in het gewricht die het gewricht volledig scheiden van de schacht. Deze indeling zorgt dat onderzoekers over hetzelfde type breuk praten. We hebben getest hoe betrouwbaar deze indeling is. Een internationaal cohort van 65 artsen classificeerde 96 verschillende distale radius fracturen. Er was minder overeenstemming over type $B$ breuken dan over type $A$ en $C$ breuken. Ook waren groepen uit verschillende landen het minder met elkaar eens over type B breuken. Als onderzoekers type $B$ breuken onderzoeken moeten ze nauwkeurig beschrijven hoe ze deze breuken hebben geclassificeerd. 


\section{DEEL 2: BEHANDELING}

Als patiënten een verplaatste breuk hebben, wordt deze normaal gesproken rechtgezet op de Spoed Eisende Hulp. Als dit niet goed lukt, of als de breuk een grote kans heeft om weer te verplaatsen, kan de patiënt kiezen voor een operatie om een geknikte pols te voorkomen (Figuur 3).

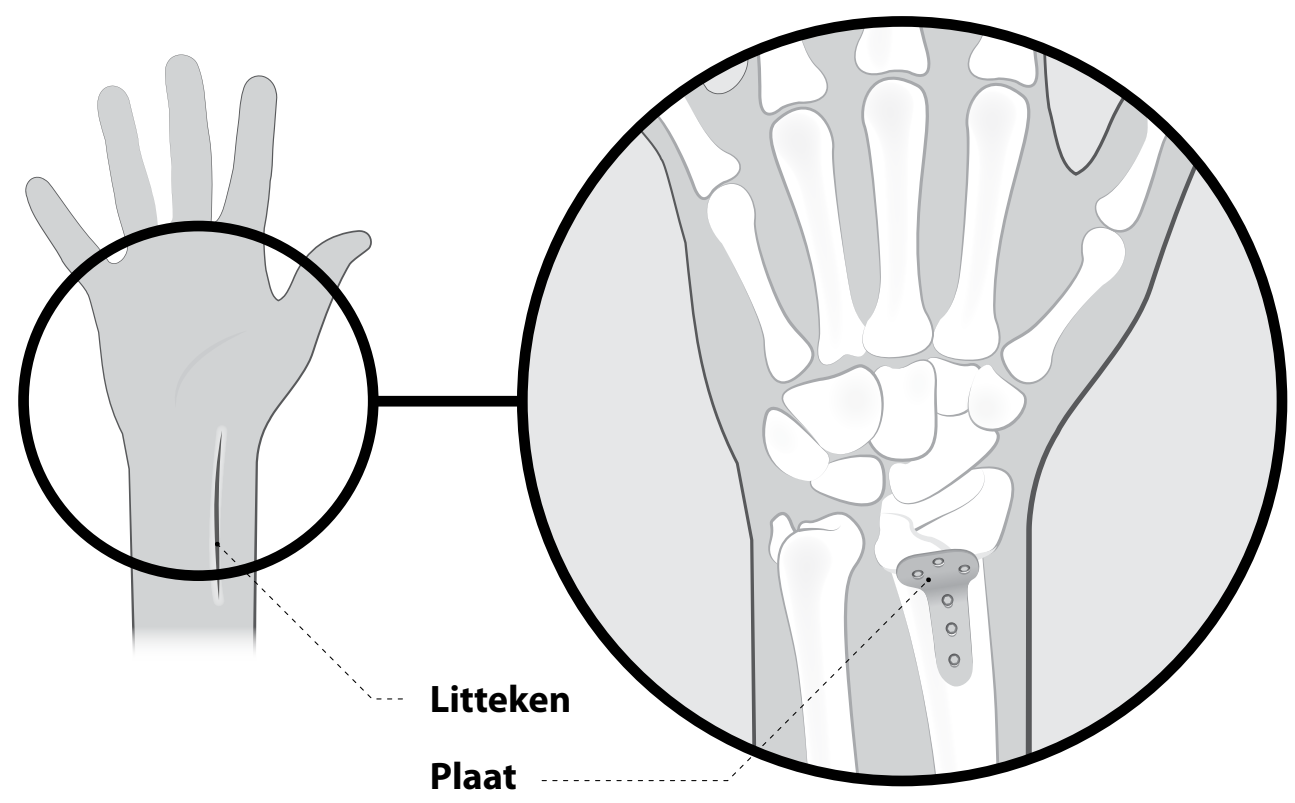

FIGUUR 3. DISTALE RADIUS FRACTUUR RECHTGEZET MET EEN PLAAT.

Om patiënten te helpen met kiezen tussen een operatie of gips, hebben we een keuzehulp ontwikkeld (www.keuzehulp.info/pp/gebrokenpols). Bij sommige type breuken kunnen de patiënt en chirurg kiezen voor een operatie, onafhankelijk van wat het rechtzetten bereikt. Toch zetten we de breuk vaak recht, omdat we denken dat dit druk op de huid en zenuw vermindert. Maar we weten niet of dit ook echt schade voorkomt. Het rechtzetten is namelijk ook pijnlijk. We onderzochten of als we de breuk niet rechtzetten dit zou leiden tot meer complicaties en meer operaties. We vergeleken 102 patiënten bij wie de breuk niet was rechtgezet met 1409 patiënten bij wie dit wel was gebeurd voor hun operatie. We vonden geen verschil tussen beide groepen in complicaties of het aantal extra operaties. Als patiënten kiezen voor een operatie voordat hun breuk is rechtgezet, en ze hebben geen problemen met hun huid of zenuw, dan is het veilig om het rechtzetten achterwege te laten.

Het doel van een operatie is om de botdelen weer op de goede plek te zetten. Het is belangrijk dat de botdelen daarna ook op hun plek blijven. We hebben gekeken of de botfragmenten verplaatst waren één jaar na een operatie met een plaat. We vonden een verschil in meerdere metingen op röntgenfoto's en CT scans. Maar de verschillen waren klein, meestal minder dan 2 millimeter of graden. Slechts één van de 66 patiënten had meer dan 3 millimeter of graden verplaatsing in verschillende metingen. Deze veranderingen zijn zo klein, dat ze ook door onnauwkeurigheid in de metingen veroorzaakt kunnen worden. We denken daarom dat na een operatie met een plaat de botdelen grotendeels op hun plek blijven. In dezelfde studie keken we of twee 
rijen schroeven in de plaat beter zijn dan één rij. We vonden onder patiënten geen verschil in polsbeweging, grijpkracht, of beperkingen. Het is goedkoper, sneller en veiliger om slechts één rij schroeven te gebruiken. Als je meer schroeven gebruikt duurt dit langer, en heb je een grotere kans om een schroef verkeerd te plaatsen.

"Hoe verder de botdelen uit elkaar blijven staan, hoe slechter de functie van de arm", denken veel artsen en patiënten. Deze manier van denken is in lijn met het biomedische model. In dit model is wat je voelt, of hoe sterk je lichaam is aangedaan, volledig gerelateerd aan de ernst van het letsel. Om dit te testen hebben we gekeken of de overgebleven verplaatsing van de botfragmenten na een operatie geassocieerd was met beperkingen, beweging en grijpkracht, één jaar een operatie met een plaat. Hierbij moet men bedenken dat alle patiënten geopereerd zijn, dus dat de overgebleven verplaatsing niet heel groot was. Maar op welke manier we het ook maten, verplaatsing was niet geassocieerd met meer beperkingen, minder beweging of minder grijpkracht. Het lijkt dat er andere factoren zijn die de functionele uitkomst na een operatie bepalen.

\section{DEEL 3: HERSTEL}

Het bio-psychosociale model houdt ook rekening met gedachten, emoties en gedrag. Dit model verklaart mogelijk beter het resultaat na een distale radius fractuur operatie dan het biomedische model. We ontdekten dat 6 weken na de breuk catastrofaal denken - de neiging om je voor te bereiden op het ergste, en je af te schermen als je pijn hebt - de belangrijkste verklaring voor stijve vingers was na een operatie. Catastrofaal denken veroorzaakt stijve vingers en huidveranderingen (zwelling, glanzende huid, verandering in haargroei) door het ontzien van de hand en pols. Labels zoals complex regionaal pijn syndroom medicaliseren mogelijk normaal ziektegedrag na een trauma. We zouden kunnen onderzoeken of ondersteuning bij het omgaan met catastrofaal denken mensen sneller helpt te herstellen.

Toen we psychologische factoren onderzochten, ontdekten we dat symptomen van depressie en catastrofaal denken (maar niet bezorgdheid over je gezondheid) verbeteren gedurende het herstel na een letsel. Wanneer we psychologische factoren gebruiken in onderzoek moeten we rekening houden met de herstelfase van patiënten.

Mensen in de Verenigde Staten en Canada gebruiken het grootste gedeelte van de wereldproductie aan opiaten. Het lijkt erop dat opiaten hun tevredenheid met pijnstilling niet verbetert. We bestudeerden welke factoren geassocieerd zijn met het vragen om meer opiaten na een operatie van een distale radius fractuur. We vonden dat mannelijk geslacht en meer geknikte breuken een significante, maar kleine, associatie hebben met meer opiaatgebruik. Eerder onderzoek heeft laten zien dat mensen met een gebroken enkel in Nederland minder opiaten gebruiken dan Amerikanen, maar dat hun pijn en tevredenheid met pijnstilling niet verschilt; dat opiaatgebruik 1 maand na het trauma geassocieerd is met depressie en post-traumatische stress; en dat meer opiaten geassocieerd zijn met meer pijn en minder tevredenheid met pijnbestrijding. Psychosociale factoren (stress, angst en minder effectieve coping) lijken essentieel in effectieve pijnbestrijding. 


\section{CONCLUSIES}

1. Bij een operatie van een distale radius fractuur lijkt het uitlijnen van het volaire fossa lunatum fragment met het styloideus radii fragment het belangrijkste onderdeel van de operatie. Misschien is het dus niet nodig om een aparte incisie te maken om ook het dorsale fossa lunatum fragment recht te zetten.

2. Type $B$ fracturen zijn moeilijk om te classificeren. Als onderzoekers type B breuken onderzoeken moeten ze nauwkeurig beschrijven hoe ze deze breuken hebben geclassificeerd.

3. Patiënten kunnen een keuzehulp gebruiken om te kiezen tussen een operatie of gips. Als patiënten kiezen voor een operatie voordat hun breuk is recht gezet, en er staat niet te veel spanning op de huid of zenuw, dan is het veilig om het zetten van de breuk achterwegen te laten.

4. Na een operatie met een plaat blijven de botdelen grotendeels op hun plaats.

5. Eén rij schroeven is net zo goed als twee rijen.

6. Hoe ver de botdelen na een operatie uit elkaar blijven staan, beïnvloedt de beperkingen, beweging en knijpkracht na één jaar niet.

7. Meer catastrofaal denken veroorzaakt stijvere vingers na een operatie.

8. Symptomen van depressie en catastrofaal denken, maar niet de bezorgdheid om je gezondheid verbeteren gedurende het herstel na een trauma.

9. Hoe ver de botten uitelkaar staan heeft maar beperkt invloed op hoeveel opiaten patiënten nemen. 
Thanks \& Recognition 
Prof. D. Ring, MD, PhD, dear David, you're a great scientist, a great doctor, but above all a great person. My role model, I can't thank you enough.

Prof. dr. M. Kon, dear Moshe, thank you for being my promotor and helping me out with the Dutch side of things.

Dr. A. Schuurman, dear Arnold, thank you for believing in me from the moment we first met all those years ago, and the opportunities you gave me.

Prof. J. Jupiter, MD, PhD, dear Jesse, thank you for your support on various studies included in this thesis. During our journal clubs I found out you're both the best quarterback and central midfielder on the MGH Hand \& Upper Extremity Service.

Dr. L.P. van Minnen, dear Paul, thank you for your invaluable advice and guidance through dire straits.

Stein Janssen, paranymph, colleague and friend, l'll have to be married before l'll spend more time with a single person.

Pieter Haasnoot, paranymph, travel companion and friend, it all started in South Africa where you taught me that with the right attitude there's no mountain too high.

The review committee, thank you for your time and interest in this thesis.

Thanks to all co-authors and other people involved in the research presented in this thesis. Alone we can do so little; as a team we can do so much more.

Koen Kerkhof and his Studio Springstof, thank you for all your great figures and illustrations, and your patience with me while we created them.

The Boston crew, thanks for all the awesome times in and around Bean town. Wald, you introduced me to American sports. Dirk, you taught me all about 3D modeling. Meijer, for your endless Slicering. Nota, how could I have finished this thesis without your Dark 'N Stormies? Or without an Old Fashioned for that matter, PJ? Niels, one of the few who dared to challenge the Harvard stadium with me. Bart, crossfit hero, my regularly sore muscles I dedicate to you.

Mom and dad, thanks for your unconditional love and infinite support.

Marija, against all odds, we traveled land and sea to be together. Thank you for your endless love and affection. 
Report of scholarship 
1. N.Stoop, T. Teunis, D. Ring, K.R. Eberlin. Variation in the rate of surgery for ulnar collateral ligament injury of the metacarpophalangeal joint of the thumb. Accepted Hand $N Y$

2. R.J. de Muinck Keizer, D.T. Meijer, B. van der Gronde, T. Teunis, S.A. Stufkens, T. Schepers, J.C. Goslings, G.M. Kerkhoffs, J.N. Doornberg. Articular Gap and Step-Off Revisited: 3D Quantification of Posterior Malleolar Fragment Reduction. Accepted Journal of Orthopaedic Trauma

3. P. Jayakumar*, T. Teunis*, B. Bravo Giménez, F. Verstreken, L. Di Mascio, J. Jupiter. AO Distal Radius Fracture Classification: Global Perspective on Observer Agreement. Accepted Journal of Wrist Surgery

4. A. Finger, T. Teunis, M.C. Hageman, E.R. Thornton, V. Neuhaus, D. Ring. Optional Follow-Up Visits for Common, Low-Risk Arm Fractures. Accepted Injury

5. T. Teunis, E.R. Thornton, T.G. Guitton, A.M. Vranceanu, D. Ring. Coaching of patients with an isolated minimally displaced fracture of the radial head immediately increases range of motion. Accepted Journal of Hand Therapy

6. T. Teunis, N.H. Bosma, B. Lubberts, D. Ter Meulen, D. Ring. Melone's concept revisited: 3D quantification of fragment displacement. Accepted Journal of Hand and Microscurgery

7. A.C. Döring, C. Overbeek, T. Teunis, S.J. Becker, D. Ring. A slightly dorsally tilted lunate on MRI can be considered normal. Accepted Archives of Bone and Joint Surgery

8. S.J. Janssen, T. Teunis, F.J. Hornicek, N. van Dijk, J.A. Bramer, J.H. Schwab. Outcome After Fixation of Metastatic Proximal Femoral Fractures: A Systematic Review of 40 Studies. J Surg Oncol. 2016 Jul 3.

9. S.J. Becker, T. Teunis, D. Ring, A.M. Vranceanu. The trapeziometacarpal arthritis questionnaire; Development and preliminary validation. Hand (N Y). 2016 Jun; 11 (2):197-205.

10. T. Teunis, A. Finger, D. Ring. What Improvement in Function and Pain Intensity is Meaningful to Patients Recovering from Low-Risk Arm Fractures? Harvard Orthopaedic Journal. 2016 Jun; 1 7(1):43-48.

11. T. Teunis, D. Ring. Comprehensive Outcome Assessment After Distal Radius Fracture. J Hand Surg Am. 2016 Jun 11.

12. M. Kuntz, T. Teunis, J. Blauth, D. Ring. Time from Booking Until Appointment and Healthcare Utilization in Hand Surgery Patients with Discretionary Conditions. J Hand Microsurg. 2015 Dec;7(2):268-275.

13. T. Teunis, S.J. Janssen, T.G. Guitton, A.M. Vranceanu, B. Goos, D. Ring. Surgeon personality is associated with recommendation for operative treatment. Hand (N Y). 2015 Dec;10(4):779-84.

14. T. Teunis, N. Stoop, C.J. Park, D. Ring. What factors are associated with a second opioid prescription after plating of the distal radius fracture? Hand (N Y). 2015 Dec;10(4):639-48.

15. T. Teunis, S.J. Janssen, T.G. Guitton, D. Ring, R. Parisien. Do orthopaedic surgeons acknowledge uncertainty? Clin Orthop Relat Res. 2015 Nov 9.

16. T. Teunis, F. Mulder, S.P. Nota, L.W. Milne, G.S. Dyer, D. Ring. No Difference in Adverse Events Between Surgically Treated Reduced and Unreduced Distal Radius Fractures. J Orthop Trauma. 2015 Nov;29(11):521-5.

17. N.H. Bosma, T. Teunis, K.R. Eberlin, J.B. Jupiter. Lower limb replantation after guillotine amputation: A 29-year follow-up. J Reconstr Microsurg. 2015 Nov;31(9):681-3.

18. S.J. Janssen, T. Teunis, E. van Dijk, M.L Ferrone, J.H. Shin, F. Hornicek, J.H. Schwab. Validation of the Spine Oncology Study Group Outcomes Questionnaire to assess quality of life in patients with metastatic spine disease. Spine J. 2015 Aug 5. pii: S1529-9430(15)01197-3.

19. S.J. Janssen, T. Teunis, D. Ring, J.H. Herndron. Orthopaedic Surgeons' View on Strategies for Improving Patient Safety. J Bone Joint Surg Am. 2015 Jul 15;97(14):1173-86. 
20. M.J. Russchen, A.R. Kachooei, T. Teunis, D. Ring. Acute proximal row carpectomy after complex carpal fracture dislocation. J Hand Microsurg. 2015 Jun;7(1):212-5

21. S. Golkari, T. Teunis, D. Ring, A.M. Vranceanu. Changes in depression, health anxiety and pain catastrophizing between enrollment and 1 month after a radius fracture. Psychosomatics. 2015 Mar 31. pii: S0033-3182(15)00061-4

22. S.J. Janssen, T. Teunis, T.G. Guitton, D. Ring. Do Surgeons Treat Their Patients Like They Would Treat Themselves? Clin Orthop Relat Res. 2015 May 9

23. T. Teunis, A.G. Bot, E.R. Thornton, D. Ring. Catastrophic Thinking is Associated with Finger Stiffness After Distal Radius Fracture Surgery. J Orthop Trauma. 2015 Apr 8

24. T. Teunis, D. Ring. Recovery from Distal Radius Fracture. J Hand Surg Am. 2015 Jan;40(1):190

25. T. Teunis, E.R. Thornton, P. Jayakumar, D. Ring. Time seeing a hand surgeon is not associated with patient satisfaction. Clin Orthop Relat Res. 2014 Dec 5

26. T. Teunis, B. Lubberts, B.T. Reilly, D. Ring. A Systematic Review and Pooled Analysis of the Prevalence of Rotator Cuff Pathology with Increasing Age. J Shoulder Elbow Surg. 2014 Dec 23, 1913-1921

27. S.J. Janssen, T. Teunis, F.J. Hornicek, J.A.M. Bramer, J.H. Schwab. Outcome of operative treatment of metastatic fractures of the humerus: a systematic review of twenty three clinical studies. Int Orthop. 2014 Nov 16.

28. T. Teunis, M. Beekhuizen, G.J.V.M. van Osch, L.B. Creemers, A.H. Schuurman, L.P. van Minnen. Soluble mediators in posttraumatic wrist and primary knee osteoarthritis. Arch Bone Jt Surg. 2014;2(3):146-150

29. S.J. Becker*, T. Teunis*, J.T. Kortlever, J. Blauth, G.S. Dyer, D. Ring. Medical Services and Associated Costs Vary Widely Among Surgeons Treating Patients With Hand Osteoarthritis. Clin Orthop Relat Res. 2014 Aug 30

30. T. Teunis, S.P. Nota, J.H. Schwab. Do corresponding authors take responsibility for their work? A covert survey. Clin Orthop Relat Res. 2015 Feb;473(2):729-35

31. J. Chan, T. Teunis, D. Ring. Prevalence of Triangular Fibrocartilage Complex Abnormalities Regardless of Symptoms Rise with Age: Systematic Review and Pooled Analysis. Clin Orthop Relat Res. 2014 Aug 5

32. S. Janssen, T. Teunis, D. Ter Meulen, M.G.J.S. Hageman, D. Ring. Estimation of Base of Middle Phalanx Size Using Anatomical Landmarks. J Hand Surg Am. 2014 Aug;39(8):1544-8

33. T. Teunis, S.P. Nota, S.A. Lozano-Calderón, F.J. Hornicek, J.H. Schwab. Outcome after reconstruction of the proximal humerus for tumor resection: a systematic review. Clin Orthop Relat Res. 2014 Jan 28

34. T. Teunis, M. Beekhuizen, M. Kon, L.B. Creemers, A.H. Schuurman, L.P. van Minnen. Inflammatory mediators in posttraumatic radiocarpal osteoarthritis. J Hand Surg Am. 2013 Aug 6

35. T. Teunis, M.R. Heerma van Voss, M. Kon, J.F.M. Macaré van Maurik. CT-angiography prior to DIEP flap breast reconstruction: a systematic review and meta-analysis. Microsurgery. 2013 Jul 9

36. A.H. Schuurman, T. Teunis. A new total distal radioulnar joint prosthesis: functional outcome. J Hand Surg Am. 2010 Oct;35:1614-1619 
Review Committee 
PROF.DR. R.L.A.W. BLEYS

Clinical Anatomy Department

University Medical Center

Utrecht, The Netherlands

PROF.DR. L.P.H. LEENEN

Surgery Department

University Medical Center

Utrecht, The Netherlands

PROF.DR. W.A. VAN KLEI

Anesthesiology Department

University Medical Center

Utrecht, The Netherlands

PROF.DR. M.J.P.F. RITT

Plastic, Reconstructive and Hand Surgery Department

Free University Medical Center

Amsterdam, The Netherlands

PROF.DR. M. MAAS

Radiology Department

Amsterdam Medical Center

Amsterdam, The Netherlands 
About the author 
The author of this thesis, Teun Teunis, was born on July 20, 1989 in Veghel, The Netherlands.

After graduating Gymnasium Bernrode cum laude in 2006, he enrolled in the Utrecht University Medical School the same year. His surgical interests were sparked early after a general surgery internship at the Medical Education Center of the Chiang Rai Regional Hospital in Thailand in 2008. He started his first upper extremity research project the year after, and has been involved in research ever since. His work won him the award for best podium presentation by the Dutch Society for Plastic Surgery in 2012, among other prizes. That year Teun was involved in developing the "should I see a doctor?" application that helps patients decide if they need to go to a general practitioner or not. The application was subsequently awarded the best health application and audience award the next year. After graduating medical school cum laude in 2013, he started his PhD research fellowship at the Hand \& Upper Extremity Service of the Massachusetts General Hospital and the Harvard Medical School in Boston. During his time in Boston Teun developed a special interested in shared decision making and decision aids, tools for patients that facilitate the medical decision making process. Together with his friend and colleague Michiel Hageman he launched the PATIENT+ foundation, dedicated to implementing shared decision making in the Netherlands and abroad. In December 2015 Teun started his general surgery residency at the OLVG in Amsterdam. In 2017 he will continue his residency training at the Plastic, Reconstructive and Hand Surgery Department of the University Medical Center Utrecht. 


\section{PROPOSITIONS}

"Based on estimated probability of loss of alignment with non-operative treatment, the surgeon offers and the patient determines their preferences for operative or non-operative treatment." This thesis
"Routinely using two rows of screws [when using a volar plate] seems to add unhelpful costs, a longer duration of surgery, and more opportunities for a misplaced or overly long screw."

This thesis

"Labels such as complex regional pain syndrome and reflex sympathetic dystrophy may be social constructions: entities that exist only because a society behaves as if they exist - as opposed to diseases like influenza and lung cancer. Labeling patients with these illness constructions is probably unhelpful."

"Patients with fractures choosing operative treatment based on the initial post-injury radiographs can avoid closed reduction when surgery is planned."
"Out of many radiographic fracture characteristics we found none to be associated with change in subjective and objective outcome 1 year after surgery." 
"Coping strategy [...]

might be one of the most

important factors determining

functional outcome after surgery."

This thesis
"When you get where you're goin', don't forget to turn

back around and help

the next one in line."

From Humble and Kind by Tim McGraw

"Some surgeons use evidence

in the same way a drunk uses a lamppost:

for support rather than illumination."

"Joe" Schwab, after Andrew Lang

"You start with a bag full of luck

and an empty bag of experience.

The trick is to fill the bag of

experience before you empty

your bag of luck."
"I don't count my sit-ups. I only start counting when it starts hurting. When I feel pain, that's when I start counting, because that's when it really counts."

Muhammad Ali

"Be inclusive, not exclusive." 
